\title{
Heuristische \\ Entscheidungen in Gruppen bei der Personalauswahl
}

Dissertation zur Erlangung des Doktorgrades der Sozialwissenschaftlichen Fakultät der

Georg-August-Universität Göttingen

vorgelegt von

Anne-Kathrin Auer, geb. Elsner

geboren am 08.06.1980 in Uelzen

Göttingen, 2016 



\section{Betreuungsausschuss}

Erstbetreuerin: Prof. Dr. Margarete Boos, Abteilung Sozial- und Kommunikationspsychologie, GeorgElias-Müller-Institut für Psychologie

Weitere Betreuer:

Prof. Dr. Steffen Kühnel, Methodenzentrum Sozialwissenschaften

Prof. Dr. Jürgen Kädtler, Soziologisches Forschungsinstitut Göttingen (SOFI)

Tag der mündlichen Prüfung: 22.02.2016 

Inhaltverzeichnis

1. Einleitung und Zielsetzung 1

1.1. Entscheidungsforschung $\quad 7$

1.2. Umdenken der Entscheidungsforschung $\quad 10$

2. Entscheidungsfindung in Organisationen 17

2.1. Merkmale von Organisationen 21

2.2. Organisation und Entscheidungsmuster $\quad 31$

2.3. Entscheidungsprozesse und entscheidungstheoretische Grundlagen $\quad 34$

2.3.1. Kriterien zur Darstellung des

Entscheidungsprozesses

36

2.3.2. Der Entscheidungsprozess

2.3.2.1. Entscheidungen nach dem MülleimerModell

2.3.2.2. Entscheidungsdefekte von Gruppen im Mülleimer-Modell

2.4. Zusammenfassung

85

3. Entscheidungsfindung von Gruppen $\quad 87$

3.1. Erforschung von Gruppenentscheidungen

89 
3.1.1. Abgrenzung von Einzel- und

Gruppenentscheidungen

3.1.2. Besonderheiten des kollektiven

Entscheidungsakteurs

3.1.3. Komplexe Entscheidungen

3.1.4. Bewertung von Entscheidungen

119

3.2. Entscheidungen auf Gruppenebene

3.3. Heuristiken im Allgemeinen

135

3.3.1. Qualität heuristischer Entscheidungen

3.3.2. Übertragbarkeit von Heuristiken auf die Gruppenebene

3.3.3. Soziales Verhalten der Gruppe

3.3.3.1. Informationsverarbeitung

3.3.3.2. Hidden Profile

3.3.3.3. Cognitive Maps

3.4. Beispiele für ausgewählte Defekte in Gruppen

3.4.1. Verantwortungsdiffusion

3.4.2. Kontrollillusion 
4. Anwendungsbeispiel: Heuristische

Entscheidungen bei der Personalauswahl

4.1. Personalauswahl als Gruppenentscheidung 252

4.2. Einfluss der Organisation bei der

Personalauswahl

260

4.3. Qualität und Rahmen der

Personalentscheidung

264

4.4. Personaldiagnostische Prozesse und ihre Hindernisse

4.4.1. Teilnehmende

285

4.4.2. Teilaktivitäten

291

4.5. Toolbox der Personalauswahl

295

4.5.1. Die Kausalität von Information und Prognose

303

4.5.2. Heuristische Entscheidungsregeln in der Personalauswahl

4.5.2.1. Verfügbarkeitsheuristik

4.5.2.2. Rekognitionsheuristik

4.6. Fazit: Bewertung der heuristischen

Personalauswahl 
4.6.1. Heuristiken von Experten/-innen

4.6.2. Overconfidence bei Experten/-innen

4.7. Fazit: Ableitungen für die praktische Personalentscheidung

4.8. Zusammenfassung

409

Abbildungsverzeichnis

Literaturverzeichnis 


\section{Vorwort}

Es war mir ein großes Anliegen, diese Arbeit zu schreiben, nicht nur, weil sie mich persönlich wie fachlich bereichert hat, sondern auch, weil die Personalauswahl in meiner täglichen Arbeit als Personalleiterin eine große Rolle spielte. Auf die Idee zu dem Thema bin ich gekommen, weil ich mich in meiner eigenen Rolle im Auswahlgremium fragte, wie die Entscheidung für oder gegen einen/eine Bewerber/-in zustande kommt und wonach sich eine "gute" Entscheidung bemisst. Eine Antwort habe ich damals nur in der Individualpsychologie finden können mit der Beschreibung von Defekten, wie dem Halo-Effekt etc. Da aber Personalentscheidungen immer in Gremien getroffen werden, wollte ich hinterfragen, wie die Gruppe eine Personalentscheidung fällt und welche intuitiven sozialpsychologischen Faktoren dabei eine Rolle spielen, die die Entscheidung des Gremiums beeinflussen können. Die Herausforderung dabei war, die Komplexität zu begrenzen, denn Einflüsse und Kriterien, die den Prozess beschreiben, gibt es viele. Mein Ziel war es deshalb, dass die praktischen Ableitungen der Arbeit in jeden Personalauswahlprozess von Organi- 
sationen (auch kleinen) aufgenommen werden können. Hierbei sollen sie eine Hilfestellung geben, einen bestehenden Auswahlprozess besser in seiner Binnenstruktur zu gestalten. In diesem Zusammenhang untersuchte ich auch die Frage, wie professionell die Personalauswahl von Experten/-innen, wie z.B. Recruitern/-innen, wirklich bewertet werden kann.

Dafür, dass ich meine Arbeit schreiben durfte, danke ich allen voran meiner Doktormutter, Frau Prof. Dr. Margarete Boos, die mich bereits im Studium inspiriert hat und mich schon 2006 dabei unterstützte, einen Studienplatz in Australien zu bekommen. Sie hat mir geholfen, Heuristiken als Entscheidungshilfen zu sehen und nicht nur als Defekte sowie genau zu hinterfragen, was menschliches Entscheiden in Gruppen ausmachen kann.

Mein besonderer Dank gilt auch Prof. Dr. Albert Martin von der Universität Leuphana in Lüneburg, bei dem ich mit der Arbeit begonnen habe und der mir meine zahlreichen Fragen zu Beginn meiner Arbeit geduldig beantwortete und mich exzellent betreute. Im Laufe der Arbeit 
zeichnete sich jedoch ab, dass mein Thema von mir weniger betriebswirtschaftlich, sondern vielmehr psychologisch erforscht werden musste.

Die Arbeit widme ich meinen Söhnen Julius Fritz (2 1/2 Jahre) und Vincent Jonas Auer (1/2 Jahr) sowie meinem Ehemann, Dr. jur. Tim-Bastian Auer. Unsere Söhne traten während des Schreibens dieser Arbeit in mein Leben, wirbelten es komplett durcheinander und bereicherten es unendlich. Großen Dank spreche meinen Eltern aus, deren Grundvertrauen in mein Handeln mich stets getragen hat, die mich begleiteten und viel praktische Hilfe leisteten. Der größte Dank gilt allerdings meinem Ehemann. Von Unterstützung zu sprechen, trifft nicht ansatzweise das, was er für mich getan hat. Auch die Absicht, diese Arbeit zu schreiben, ist seinem Nachdruck zu verdanken, sie entstand während unserer Hochzeitsreise 2011. Ihm gilt der größte Dank für die zeitlichen Freiräume und seine unerschöpfliche Zuversicht.

Anne-Kathrin Auer 


\section{Einleitung und Zielsetzung}

Entscheidungen, die von Menschen über Menschen getroffen werden, sind selten korrekt und noch weniger rational. Der Gedanke war Anlass für die vorliegende Arbeit. Verbunden war er mit diversen konkreteren Fragestellungen, insbesondere hinsichtlich der Art und Weise, wie es zu Gruppenentscheidungen kommt, der Wirkungsweise von Entscheidungseffekten wie Heuristiken und deren Bewertungsmöglichkeiten. Den Schwerpunkt legt die vorliegende Arbeit deshalb auf das Entscheidungsverhalten von Gruppenmitgliedern im Organisationsgeschehen. $\mathrm{Zu}$ Beginn dient eine Gegenüberstellung der Annahmen bezüglich der normativen (klassischen) und der deskriptiven Entscheidungsforschung dem besseren Verständnis des Phänomens Entscheidungsverhalten in Organisationen. Insbesondere im Hinblick auf heuristische Entscheidungen wird diese Darstellung als Grundlage für die Diskussion der begrenzten Möglichkeiten einer rationalen Entscheidungsfindung benötigt.

Die Organisation stellt den Rahmen dar, in dem die Gruppenmitglieder handeln. Sie ist ein soziales Gebilde, 


\section{Einleitung und Zielsetzung}

und ihre Merkmale werden analysiert, um zu bewerten, worin die Entscheidungsfindung der Gruppenmitglieder eingebettet ist. Ihre Entscheidungsprozesse und -regeln sind verhaltenslenkend für die Gruppenmitglieder und in den Organisationsprozess integriert. Hierzu wird ein Überblick gegeben.

Das Mülleimer-Modell der Entscheidung von Cohen et al. (1972) soll das Verhalten von Organisationen im Entscheidungsprozess darstellen. Es bezeichnet das Entscheidungsverhalten in Organisationen als organisierte Anarchie. Mit ihrem „anarchischen“ Ansatz wenden die Forscher/-innen sich vom rationalen Entscheidungsverhalten in Organisationen ab. Das Zusammenspiel der vier organisationalen Ströme im Modell (namentlich: Probleme, Lösungen, Teilnehmende und Entscheidungsangelegenheiten) beschreibt das Entscheidungsverhalten als zufällig. Die vier Ströme sind unabhängig, dynamisch und treffen in der Organisation durch Zufall aufeinander. Damit kann das Modell verdeutlichen, dass das Entscheidungsverhalten ständig in Bewegung und rational begrenzt ist. Mit dem Modell soll neben dem zufälligen und dynamischen Prozess der Entscheidungsfindung in Organisationen auch die kollektive Seite der Entschei- 
1 Einleitung und Zielsetzung

dungsfindung (nämlich das Handeln der Organisationsmitglieder) betont werden.

Es werden außerdem die Komplexität und Qualität von Gruppenentscheidungen, im Vergleich zu Einzelentscheidungen, behandelt. Es stellt sich dabei die Frage, ob sich individualpsychologische Fehler auf die Ebene von Gruppenmitgliedern übertragen lassen. Hierauf hat die Forschung bislang kaum ihre Aufmerksamkeit gerichtet (vgl. Scholz et al. 2003, S. 16). Die Beantwortung dieser Aufgabenstellung soll mittels einer Auswertung der Forschungsliteratur erfolgen. Entscheidungseffekte werden meist auf individueller Ebene erforscht, jedoch wird so gut wie keine Entscheidung allein getroffen, stattdessen wird eine Entscheidung meist von sozialen Aspekten beeinflusst. Die Ergebnisse der individualpsychologischen Forschung können nicht analog für die Gruppenentscheidungssituation übernommen werden, jedoch bieten sie eine gute Analysebasis. Um Entscheidungsphänomene auf eine Gruppenebene zu übertragen, soll u.a. die Informationsverarbeitung in Gruppen im Fokus stehen. 
1 Einleitung und Zielsetzung

Das Entscheidungsverhalten in Organisationen soll organisationale Entscheidungsprozesse (der Entscheidungsforschung) mit der verhaltenspsychologischen Sichtweise von Heuristiken und sozialen Effekten verknüpfen. Die vorliegende Arbeit versucht nicht, ein optimales Entscheidungsverhalten darzustellen, sondern vielmehr soll aufgezeigt werden, ob heuristische Entscheidungen fehlerhaft oder in der Entscheidungspraxis auch für Gruppen eine sinnvolle Entscheidungshilfe sein können. Das Phänomen der heuristischen Entscheidungen in Gruppen soll dabei der Mittelpunkt der Diskussion sein. Hierbei handelt es sich um ein psychologisches Phänomen, bei dem Gruppen in ihrer Entscheidungsfindung einfache, aber effiziente (fast and frugal) Lösungswege wählen, weil innen nur unvollständige Informationen zur Verfügung stehen.

Grundsätzlich stellt sich die Frage, ob Heuristiken sich dazu eignen, das Konzept der heuristischen Entscheidungsfindung auch auf Gruppen (kollektive Ebene) zu übertragen, ohne als Summe der "Bauch“Entscheidungen einzelner Organisationsmitglieder zu gelten. Konkret gesagt, soll dargestellt werden, ob Heuristiken in einem sozialen Umfeld wie der Gruppe mehr 
1 Einleitung und Zielsetzung

sind als nur einzelne Aktivitäten und Denkleistungen und welche heuristischen Dynamiken es geben kann, welche die Entscheidungen der Gruppe beeinflussen. Es sollen also nicht nur einzelne Entscheidungen für eine Gruppenentscheidung addiert werden. Vielmehr bildet gerade die soziale Dynamik der Gruppe das besondere „Salz in der Suppe“, welches das heuristische Entscheidungshandeln der Gruppenmitglieder beeinflusst.

Mithilfe der heuristischen Entscheidungstheorie, die auf den Konzepten Gigerenzers und der ABC-Gruppe (Adaptive Behavior and Cognition) (1999) sowie Tverskys und Kahnemans (1974) basiert, soll in der besonderen Entscheidungssituation der „Personalauswahl“ die Wirkweise von Heuristiken der Gruppenmitglieder analysiert werden. Die Personalauswahl ist für die Anwendung der Kernfragestellung dieser Arbeit besonders geeignet. Grund hierfür ist die Annahme, dass bei Einstellungsgesprächen von Organisationen Gruppenheuristiken häufig Gebrauch finden und die Personalauswahl meist von mehreren Personen getroffen wird. Außerdem handelt es sich bei der heuristischen Entscheidung um ein Phänomen von organisationaler Tragweite (z.B. weil Personaleinstellungen weitreichende Folgen haben können), mit 
1 Einleitung und Zielsetzung

welchem die Einflüsse der Organisation auf das Entscheidungsverhalten in Gruppen dargestellt werden können. Ausgewählte Heuristiken werden dazu betrachtet, z.B. aus der adaptiven Toolbox von Gigerenzer et al. (1999a), wie die Take-the-best-Heuristik und die Rekognitionsheuristik, sowie die Verfügbarkeitsheuristik von Tversky und Kahneman (1974). Diese Heuristiken sollen daraufhin untersucht werden, in welcher Form sie als kollektive Phänomene bei der Personalauswahl in Erscheinung treten, d.h., wie sie im Entscheidungsprozess der Personalauswahl wirken und wie ein Zustandekommen von Heuristiken auf Gruppenebene zu erklären ist.

Experten/-innen des Personalauswahlprozesses sind eine besondere Personengruppe im Entscheidungsverhalten. Es stellt sich die Frage, nach welchen Entscheidungsregeln sie ihre Entscheidungen treffen. Außerdem stufen sie sich selbst oft als sehr kompetent im Hinblick auf ihre Entscheidungsqualität ein. Dabei spielen das Feedback der Organisation nach der Einstellung sowie die Kausalität der Entscheidung zur Bewerberleistung eine große Rolle. Ferner wird die Frage relevant, ob auch Experten/-innen heuristisch entscheiden. 


\subsection{Entscheidungsforschung}

In der Arbeit wird die Brücke zwischen individuellem und kollektivem Entscheidungsdenken geschlagen. Das Ziel liegt in der Verknüpfung von heuristischem Entscheidungsverhalten von Individuen mit der Frage, ob auch Gruppen heuristisch handeln. Wie dies in der Organisation Gestalt annimmt, wird anhand der Entscheidungssituation „Personalauswahl“ Anwendung finden.

\subsection{Entscheidungsforschung}

Die Entscheidungsforschung hat im Laufe der Zeit eine interessante Wandlung erfahren, die im Folgenden dargestellt werden soll. Den Anfängen vieler wissenschaftlicher Disziplinen, wie den Politikwissenschaften oder der (Arbeits-)Psychologie, lag der Gedanke der Rationalität, welcher auf der „Homo oeconomicus“-Forschung basiert, zugrunde: Der Mensch entscheidet rational und wählt zwischen Handlungsalternativen diejenige aus, die seiner rationalen Präferenzordnung am besten entspricht (vgl. Wossidlo 1975, S. 102 ff.). Von diesem Tenor wird auch die Entscheidungsforschung beeinflusst, und aus ihren Anfängen entstand die normative Entscheidungsforschung, die eng mit der Theorie der Unternehmung ver- 


\subsection{Entscheidungsforschung}

knüpft ist. Deren Annahme ist es, dass Organisationen in einem vollkommenen Markt tätig sind und das Nettoeinkommen bei fixen Preisen und Produktionsfunktion maximiert wird, dass es also das Ziel ist, die optimale Kombination der Produktions- und der Einsatzmenge zu finden (vgl. Cyert/March 1995, S. 5). Zur Theoriebildung war diese Grundannahme geeignet. Sie lässt aber generell die Tatsache des irrationalen menschlichen Verhaltens und unbedachter Entscheidungen außer Acht. Dennoch bildet das Idealbild des Homo oeconomicus mit seinem Streben nach Optimierung die Grundlage für alle weiteren Forschungsansätze. Angemerkt werden sollte, zu welcher Zeit diese Überlegungen entstanden sind, die großen Einfluss auch auf einige andere Theorieentwicklungen hatten: Im Zeitalter der Industrialisierung ermöglichte das Konzept der Arbeitsteilung eine tayloristisch gewinnbringende Massenproduktion. Die Absatzraten waren weitgehend stabil und die Märkte geschlossen, sodass die Umwelt abgesteckt war und auch interne Entscheidungen rational zu bewältigen waren. Vor diesem Hintergrund war ein rationales, nutzenmaximierendes Handeln denkbar.

Die frühe Entscheidungsforschung wollte Entscheidun- 


\subsection{Entscheidungsforschung}

gen von Individuen greifbar und für die Betriebswirtschaft nutzbar machen. Sie bemühte sich, die Kluft zwischen Wunsch und Wirklichkeit darzustellen und mit Theorien die Praxis der Entscheidungsfindung und ihre Wirkung zu erklären. Sie klassifizierte die Entscheidungsprozesse nach Phasen und unternahm den Versuch, die Bewertung der Probleme zur Suche nach geeigneten Lösungsmethoden ex post mathematisch darzustellen. Dies ist schon deshalb keine leichte Aufgabe, weil es häufig keine klar voneinander abgrenzbaren Entscheidungsalternativen gibt (vgl. Martin 2001, S. 6).-Hintergrund war, dass Fehlentscheidungen vermieden werden sollten, und so war man auf der Suche nach Maßstäben, um die Entscheidung als Phänomen der Organisationen einzuordnen. Organisationen wurden als soziale Systeme betrachtet, die zielorientiert bzw. absichtsgeleitet Entscheidungen von ihren Teilnehmenden treffen lassen (vgl. Martin 1995, S. 7). Herausforderungen, denen heutige Organisationen gegenüberstehen, sind komplexerer Natur und manchmal wenig absichtsgeleitet bzw. nicht im Sinne der Organisation, sodass die Schätzungen, die sich aus den frühen Entscheidungsverhaltenstheorien ergaben, eher unpräzise sind und zudem nur eine be- 
1.2 Umdenken der Entscheidungsforschung

grenzte Auswahl von Entscheidungsalternativen betrachtet wurden (vgl. Cyert/March 1995, S. 95).

Die ökonomische Wissenschaft nahm bis zu den 1950erJahren an, dass die Mitglieder der Organisation, also die einzelnen Organisationsteilnehmenden, organisatorische Ziele teilten (vgl. Scholl 2004, S. 515). Dieser Grundgedanke ist auf den ersten Blick organisationsseitig wünschenswert, weil er Entscheidungsprozesse vereinfacht, jedoch unter Gesichtspunkten der Diversität von Individuen sowie der daraus entstehenden kreativen Meinungsvielfalt nicht praktikabel oder gar realistisch ist. Auch aus Gründen der Vielfalt von Entscheidungsalternativen und damit einhergehend der Komplexität des Denkens ergeben sich Schwierigkeiten für die Vorhersagbarkeit menschlichen Handelns. Trotzdem suchte die Entscheidungsforschung nach einer Darstellung des Entscheidungsverhaltens sowohl von Individuen als auch von Organisationen.

\subsection{Umdenken der Entscheidungsforschung}

In der normativen Entscheidungstheorie ist die zentrale Annahme das Bild des Homo oeconomicus, in dem die 
1.2 Umdenken der Entscheidungsforschung

Nutzenmaximierung des Entscheidungsverhaltens sowie das rationale Entscheidungsverhalten im Fokus stehen. Der/die rationale Entscheider/-in systematisiert nach der Theorie Entscheidungssituationen für sich und leitet Regeln für eine optimale Entscheidung ab. Diese Sichtweise wurde durch die deskriptive Entscheidungstheorie abgelöst, die sich mit der Erklärung und nicht mit der normativen Beschreibung von Entscheidungsverhalten auseinandersetzt. Es wurde gefragt, wie Entscheidungen zustande kommen, und der Auswahlprozess wurde als solcher in den Mittelpunkt gerückt. Dabei wurde das Entscheidungsverhalten von Einzelpersonen betrachtet. Im Gegensatz zur Entscheidungslogik der normativen Entscheidungstheorie fokussieren die Vertreter/-innen der deskriptiven Entscheidungstheorie das tatsächlich beobachtete Entscheidungsverhalten (z.B. Demotivation, sich für die Belange der Organisation zu engagieren). Deskriptive und normative Entscheidungstheorien sind Theoriengruppen und damit Teilgebiete der entscheidungstheoretischen Bewegungen. Drei zentrale Aspekte unterscheiden die beiden Bewegungen voneinander. Diese Unterschiede sollen im Folgenden beschrieben und in Abbildung 1 dargestellt werden: 


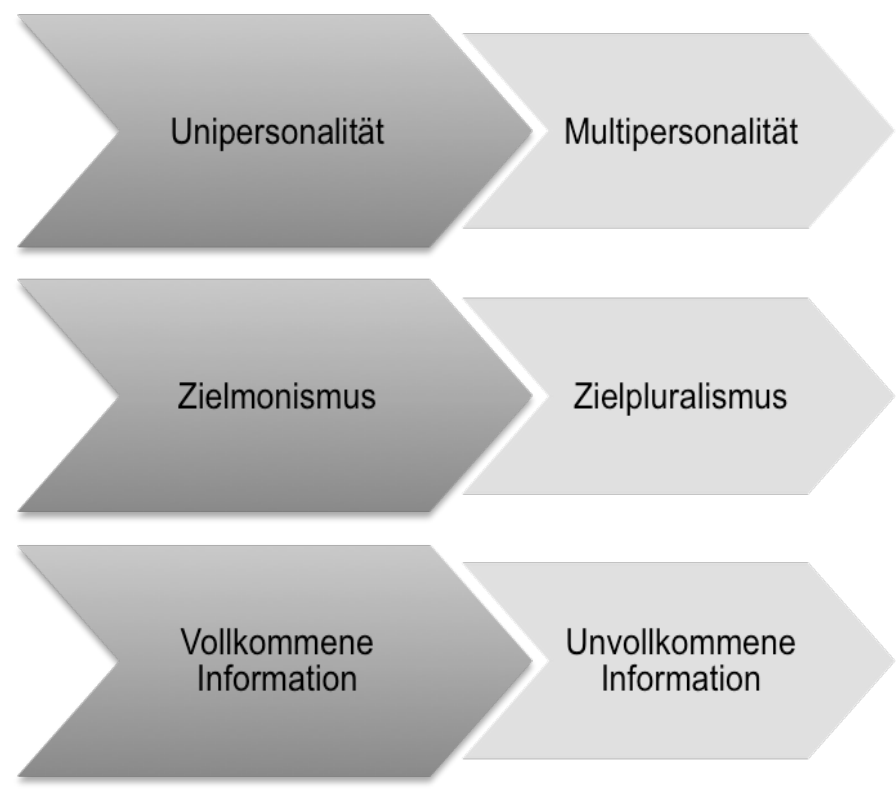

Abbildung 1: Abgrenzung von normativer und deskriptiver Entscheidungstheorie (in Anlehnung an Wossidlo 1975, S. 104).

Die Entscheidungstheorie entwickelte sich von der Unipersonalität zur Multipersonalität, ersetzte den Zielmonismus durch den Zielpluralismus und räumte gleichfalls mit der vollkommenen Informationsversorgung auf.

Von der Unipersonalität zur Multipersonalität: Entgegen den Annahmen der klassischen (normativen) Entscheidungsforschung werden Entscheidungen selten allein 


\subsection{Umdenken der Entscheidungsforschung}

getroffen. Sie sind meist kollektiver Natur, d.h., Entscheidungen werden oft gemeinsam gefällt oder haben zumindest eine kollektive Dynamik durch Beratung, Unterstützung etc. Prozesse werden vom Handeln und Denken vieler Akteure/-innen bestimmt (vgl. Schulz 1977, S. 22), und Entscheidungen werden meist in Gruppen getroffen (vgl. Martin 1998, S. 53). Entscheider/-innen ergänzen sich in ihren Meinungen und Argumenten und entwickeln gemeinsame Prozessdynamiken sowie Entscheidungsregeln. Deshalb wandelte sich auch der Fokus von der individuellen (unipersonalen) hin zu einer multipersonalen Betrachtung.

Vom Zielmonismus zum Zielpluralismus: Organisationsziele, so die Annahme der normativen Entscheidungstheorie, sind für die Organisationsmitglieder transparent und bestehen nur aus einem Ziel (Zielmonismus). Jedoch ist diese Sichtweise nicht mit dem tatsächlichen Zielpluralismus der Realität vereinbar. Organisationen und ihre Mitglieder verfolgen stets mehrere Ziele (Zielpluralismus), oft gleichzeitig, die einander sogar widersprechen können (vgl. Scholl 2004, S. 530).

Von der vollkommenen zur unvollkommenen Informationsversorgung: Der Prozess des Entscheidens ist nicht 
1.2 Umdenken der Entscheidungsforschung

notwendigerweise mit einer vollkommenen Informationsversorgung gesegnet. Im Gegenteil, selten sind Informationen vollständig verfügbar und so umfassend, dass eine rationale Entscheidungssituation entstehen kann.

Dies sind die wichtigsten Unterschiede zwischen der normativen und der deskriptiven Entscheidungstheorie. Die deskriptive Entscheidungstheorie brachte neue Erkenntnisse mit sich und setzte einen Wandel mit neuen Entscheidungstheorien und -modellen vielfältiger Art in Gang (vgl. Weinert 2004, S. 675) ${ }^{1}$.

Ein Vertreter der deskriptiven Entscheidungstheorie ist Herbert A. Simon. Für seine Erforschung der Entscheidungsprozesse in Wirtschaftsorganisationen erhielt er im Jahr 1978 den Wirtschaftsnobelpreis. In seiner Theorie der begrenzten Rationalität (1959) zeigt sich auch der Wandel in Bezug auf Entscheidungen bei folgenden Problemen (vgl. Simon 1959, S. 85):

${ }^{1}$ Siehe Ausführungen von Cyert/March 1995, S. 1. 


\subsection{Umdenken der Entscheidungsforschung}

- Menschen haben nur ein unvollständiges Wissen über die Bedingungen, die Einfluss auf die Konsequenzen von Entscheidungen nehmen.

- Sie sind nur begrenzt in der Lage, alle Entscheidungsalternativen in Betracht zu ziehen.

- Zukünftige Ereignisse können nur begrenzt vorweg bewertet werden.

Damit wurde erstmals deutlich, dass subjektive Elemente im Entscheidungsverhalten eine Rolle spielen und die Instabilität der Umgebung als zentraler Bestandteil der Entscheidung akzeptiert werden muss. Simon beschreibt Entscheidungsverhalten deshalb als beschränkt rational. Er geht davon aus, dass bei der Entscheidungsfindung die Suche nach Alternativen beendet ist, sobald man eine Lösung gefunden hat, mit der man zufrieden ist, obwohl es vielleicht eine bessere oder rationalere geben könnte. Es werden also nicht alle Entscheidungsalternativen in Betracht gezogen. Er nennt weitere Hindernisse im Entscheidungsprozess, die bei der normativen Entscheidungstheorie noch keine Beachtung fanden: Es kann im Entscheidungsprozess zu Zielkonflikten kommen, die Organisationsmitglieder entscheiden unter Un- 
1.2 Umdenken der Entscheidungsforschung

sicherheit, ihre Erwartungen spielen eine Rolle, und auch Entscheidungsergebnisse von Verhandlungen sind nur begrenzt rational (vgl. Simon 1959, S. 86). Bei inm klingt bereits das Irrationale in der Entscheidungsfindung an (vgl. Wessler 2012, S. 113).

Das Entscheidungsverhalten in Organisationen wurde von zahlreichen Forschern/-innen dargestellt, die den Prozess der Entscheidungsfindung objektivieren wollten. Die subjektiven Elemente in der organisationalen Entscheidungsfindung sind dabei besonders schwer darzustellen, weil sie eigenen Algorithmen der Bewertung folgen, die sich nicht immer erschließen. Wie Entscheidungen definiert werden und welchen Hintergrund die Entscheidungsfindung in Organisationen für die vorliegende Arbeit haben soll, wird im Folgenden dargestellt. 


\section{Entscheidungsfindung in Organisationen}

Der erste Teil der Arbeit (Kapitel 1 und 2) soll sich auf die Erkenntnisse der Entscheidungstheorie gründen. Damit stellt die institutionelle Entscheidungsforschung zunächst die Basis dar für weitere Überlegungen bis hin zur Entscheidungsfindung von Gruppen (Kapitel 3). Um die Systematik der Entscheidungsfindung in Organisationen zu konkretisieren, wird für den weiteren Verlauf eine Abgrenzung des Entscheidungsbegriffs benötigt: Was für eine Art von Entscheidungen soll analysiert werden?

Im Kern des Unternehmenshandelns stehen die Entscheidungen (vgl. Martin 2004, S. 4). Entscheidungen sind für den Erfolg des Unternehmens maßgeblich und bestimmen die Zukunft des Unternehmens wesentlich mit. Dies gilt für jede Art von Organisation, unabhängig von ihrer Größe und dem Zweck ihres Handelns. Doch welche Art von Entscheidungen betrachtet wird, darüber gibt es unterschiedliche Auffassungen. Was ist gemeint, wenn es um Entscheidungen geht? Sind Entscheidungen in Gruppen eine besondere Art des Auswählens im Vergleich zu Einzelentscheidungen? Zunächst soll das Phä- 
2 Entscheidungsfindung in Organisationen

nomen der Entscheidung grundsätzlich dargestellt werden, um anschließend das Entscheiden von Gruppenmitgliedern in Organisationen zu betrachten.

Die meisten Entscheidungen im täglichen Leben erfolgen gewohnheitsmäßig und wenig reflektiert. Man möchte einen Obstsalat machen und kauft deshalb Bananen und Äpfel ein, oder man muss eine Broschüre vervielfältigen und entscheidet sich, den Kopierer zu benutzen. Doch in Organisationen sind Entscheidungen oftmals vielschichtiger und unübersichtlicher. In den Anfängen der Entscheidungsforschung gab es bei der Definition des Entscheidungsbegriffs lediglich die Gegenüberstellung zweier Entscheidungstypen, das produktive und kreative Problemlöseverhalten und das gewohnheitsmäßige (einfache) Entscheiden (vgl. Joost 1975, S. 1).

Wie die obigen Beispiele (Obstsalat und Kopieren) zeigen, ist die gewohnheitsmäßige Auswahl zweifelsohne eine Art der Entscheidung. Jedoch hält sich der kognitive Aufwand in Grenzen und ist „schnell erledigt“. Die Abgrenzung der Gewohnheitsentscheidungen von der Problemlösung ist zu eng bemessen. Sie greift für einen wissenschaftlichen Anspruch klar zu kurz, da sie lediglich eine Klassifizierung vornimmt. Eine andere Definition, die 
2 Entscheidungsfindung in Organisationen

wissenschaftlichen Ansprüchen genügt, könnte Folgende sein:

Bei Entscheidungen in Organisationen handelt es sich um eine Auswahl, „die unter Zielbedingungen aus einer Menge von Alternativen auf der Grundlage verfügbarer Informationen getroffen wird“ (Cyert/March 1995, S. 21). Die Entscheidungsdefinition von Cyert und March weitet den Horizont der Entscheidungen aus, und es können sowohl gewohnheitsmäßige als auch komplexe Entscheidungen darunter gefasst werden. Bei der Entscheidung geht es also um den Wahlakt in der Organisation aus bekannten Alternativen, die unter Zielbedingungen die Kriterien für die Auswahl sein könnten. Diese Eingrenzung der Entscheidung führt dazu, dass lediglich die verfügbaren Informationen die Basis für die Entscheidungsfindung sind. Es lässt sich daraus die Unvollkommenheit der Perspektive der Entscheidungsforschung ablesen. Dies ist eine ganz andere Herangehensweise als die der Ökonomie zu Anfang des 19. Jahrhunderts, die eine vollkommene Informationsbandbreite unterstellte. In der organisationalen Verhaltensforschung ist die Unvollständigkeit der Information eine wichtige Stellschraube, denn die Frage, die dahinter steht, lautet, wie 
2 Entscheidungsfindung in Organisationen

Entscheidungen in ihrer Qualität verbessert werden können: Können überhaupt gute Entscheidungen getroffen, wenn doch die Basisinformationen unvollkommen sind? Diese Frage wird in der vorliegenden Arbeit von Bedeutung sein, die sich auch mit der praktischen Entscheidungsfindung auseinandersetzen wird.

Um die Bestimmung des Entscheidungsbegriffs abzuschließen, soll die kollektive Sicht von Entscheidungen in Organisationen dargestellt werden. Kirsch (vgl. 1971, S. 55) differenziert Z.B. zwischen Einstellung, Wahrnehmung, Suchverhalten und Informationsverarbeitung als den einzelnen Entscheidungskomponenten. Der kollektive Entscheidungswille setzt sich aus diesen einzelnen Komponenten zusammen. Die Entscheidungen der Gruppe sind nicht nur die Summe der Einzelentscheidungen, wie es etwa bei additiven Aufgaben (wie Tauziehen, Angeln oder Brainstorming) der Fall ist (vgl. Drewes et al. 2011, S. 224). Dies verlangt vom Betrachter einen hohen Theoretisierungsgrad und vom kollektiven Willensgeber, z.B. der Entscheidergruppe, letztlich Einigungswege, die jedoch nicht immer in eine Entscheidung münden. Damit ist gemeint, dass jedes Gruppenmitglied 
eine Entscheidung für sich trifft, aber die Summe dieser einzelnen Entscheidungen erst die Entscheidung der Gruppe ergibt. Eine Gruppenentscheidung benötigt Kommunikation, Abstimmung, Einigung oder gar Dispute, um zu einer gemeinsamen Entscheidung zu kommen. Neben dem Wahlakt des Einzelnen ist also der kollektive Einigungsweg für eine gemeinsame Entscheidung wichtig. Diese Sichtweise wird auch im nächsten Kapitel entscheidend sein, wenn es um das Konstrukt geht, in dem Entscheidungen getroffen werden: die Organisation.

\subsection{Merkmale von Organisationen}

Die Organisation spielt als Träger der Entscheidung eine wichtige Rolle. Sie ist die Umgebung, in der sich die Mitglieder entscheiden. Nach Ackoff (1961, S. 276) ist die Organisation ein selbst gesteuertes System, in dem Individuen oder Gruppen von Individuen die Verantwortung für die Wahl aus einer Anzahl von möglichen Handlungen in spezifischen Situationen haben.

Organisationen zeichnen sich durch gewisse Merkmale aus, die Auswirkungen auf den Entscheidungsverlauf und 
die Entscheidung der Gruppenmitglieder haben können.

Diese Merkmale lassen sich in fünf allgemeine, zum Teil entscheidungsbezogene Merkmale unterteilen. Folgende Einteilung zeigt eine Auswahl der Organisationsmerkmale (in Anlehnung an Ackoff 1961, S. 276 f.):

- Zweck

- Geografie

- Zeit

- Organisationskultur

- Organisationsstruktur

Ackoff (1961) zieht in seinen Ausführungen zu den Organisationsmerkmalen den Vergleich mit zu Militärentscheidungen („Wellen einer angreifenden Streitmacht" als Beispiel für Zeit, „räumliche Verantwortungsgebiete einer Armee" als Beispiel für geografische Gesichtspunkte etc.). Jedoch ist die Armee eine besondere Art der Organisation, deren Entscheidungskultur durch Hierarchien geprägt ist und der Ausdruck durch Dienstgrade verliehen wird. In einer militärischen Organisationsumgebung sind die Entscheidungspersonen nicht unabhängig und 
Entscheidungen verlaufen weniger demokratisch. Sie stellt dennoch eine selbst gesteuerte Organisation dar. An diesem Organisationsbeispiel zeigt sich aber, dass auch hier die Merkmale der Organisation die Entscheidungssituation determinieren können. Der Unterschied ist lediglich, dass in Militärorganisationen jede Diskussion durch die Autoritäten ohne offene Kommunikation oder demokratische Elemente der Mitbestimmung entschieden werden kann. Damit ist das Entscheidungsverhalten durch die Art der Organisation begrenzt. Im weiteren Gedankenspektrum der Arbeit soll das Entscheidungsverhalten der Organisationsmitglieder eher auf offen demokratische Organisationssysteme, wie Unternehmensorganisationen, zielen. Ackoffs Merkmale der Organisationen werden in der vorliegenden Arbeit deshalb um die Merkmale Organisationsstruktur und -kultur erweitert, um den Rahmen der Organisation zu konkretisieren. Zeit, Zweck und Geografie sind nicht ausreichend für die Beschreibung von Organisationen des und Organisationverhaltens. 


\section{Zweck}

Der Zweck ist nicht nur ein lebenswichtiges Merkmal der Organisation, aus dem Zweck ergeben sich auch die Blickrichtung sowie der Sinn und die Handlungsabsicht der Organisation (Gründungsgrund, Wettbewerbsvorteil, Alleinstellungsmerkmal etc.). Er stellt die Aufgabe der Organisation dar. Bei den zu treffenden Entscheidungen berücksichtigen die Entscheidenden den Zweck der Organisation, der im Entscheidungshandeln der Organisationsmitglieder Ausdruck findet. Cyert und March (vgl. 1995, S. 1) betrachten die Organisation als Filter für die Organisationsmitglieder, weil ihr funktionelles Denken organisationssoziologisch erlernt und langfristig angelegt ist. Dieses Denken prägt ihr Verhalten. Die Organisation agiert anhand von Entscheidungsmustern. Diese allgemeinen, festgelegten Entscheidungsverfahren sind langfristig erlernte kognitive Regeln und lassen sich nur schwer ändern (vgl. Cyert/March 1995, S. 118). Die Organisation ist ein anpassungsfähiges System, aber ihre allgemeinen Regeln sind konstant. 


\section{Geografie}

Bei den geografischen Gesichtspunkten geht es um die räumliche Lage der Organisation. Denn auch der Standort der Organisation spielt eine Rolle im Organisationsgeschehen. Unterschiede können sich z.B. aus verschiedenen Absatzmärkten, räumlicher Nähe zu Zulieferern, rechtlichen Gegebenheiten, Infrastruktur etc. ergeben. Hier kann zum einen von Verhaltensstandards gesprochen werden, die der Standort mit sich bringt (eng verknüpft mit der Organisationskultur). Zum anderen können aus den Standortbedingungen organisationale Unterschiede erwachsen. Dies führt auch zu Unterschieden in dem umweltbezogenen Aktionsfeld der Organisationsmitglieder. Deshalb können Entscheidungen einer Organisation von ihren geografischen Umweltbedingungen (z.B. Anpassungen auf Marktänderungen) beeinflusst werden (vgl. Cyert/March 1995, S. 22). Auch wenn die Organisation zum Beispiel mehrere Standorte hat, prägt dies ihr Verhalten, z.B. durch Mitarbeiter-Diversity, interkulturelle Kommunikation etc. 


\section{Zeit}

Der Faktor Zeit als Organisationscharakteristikum bleibt bei Ackoff (1961) weitgehend unbestimmt, wobei Zeit in vielerlei Hinsicht für die Organisation von Bedeutung ist. In welcher Zeit eine Organisation agiert, stellt gleichfalls ein Organisationsmerkmal dar. Die Zeit bringt Umstände mit sich, die für das Organisationsgeschehen entscheidend sein können. So stellt sich das Entscheidungshandeln einer Organisation in Zeiten einer Rezession anders dar als bei boomenden Absatzmärkten, oder während einer Inflation mag das Organisationshandeln von Vorsicht geprägt sein, und es erfolgen weniger Investitionen. Sogar das Jahrhundert als Grobraster spielte eine Rolle, z.B. vor dem Hintergrund des technischen Fortschritts. Auch kleine Zeiteinheiten, wie Jahreszeiten (Saison) oder beim Aktienhandel (Börsengeschäfte) sind relevant. Zeit kennzeichnet also u.a. die wirtschaftlichen Entscheidungsbedingungen der Organisation, aber auch die Technologie- bzw. Innovationsreife.

\section{Organisationskultur}

Wie bereits erwähnt, sind kulturelle Gegebenheiten oft eng an geografische Bedingungen geknüpft, denn mit der 
2.1 Merkmale von Organisationen

geografischen Lage geht auch häufig die Frage der Kultur einher. Wenn eine Organisation außerhalb ihres eigentlichen Standorts Niederlassungen (z.B. im Ausland) unterhält, können kulturelle Unterschiede zutage treten. Beispiel: McDonalds produziert in Indien keine Burger mit Rind, weil die Kultur des Landes aus religiösen Gründen Rindfleischverzehr verbietet; und so findet diese Kultur auch in die Organisation des Landes Eingang. Grundlage für die Organisationskultur sind deshalb die geteilten Werte und Normen, die sich im historischen Organisationsgebaren äußern (z.B. „Das haben wir schon immer so gemacht"). Dabei hat die jeweilige Organisationskultur eine verhaltenslenkende Wirkung auf alle Mitglieder (vgl. Jochims 2010, S. 60). Sie stellt die individuellen Besonderheiten der Organisation dar. Organisationskulturen $\mathrm{zu}$ verändern, gestaltet sich deshalb schwierig, denn sie sind oft tief in der Organisation und ihren Normen verankert (vgl. Auer-Rizzi 1998, S. 263).

\section{Organisationsstruktur}

In der Organisation können Handlungen und Prozesse durch Strukturen begrenzt werden. Sie haben verhaltenslenkenden Charakter (vgl. Fleischer 2001, S. 2), weil sie 
„Von einer weitgehend identischen Menge von Organisationsmitgliedern getragen werden" (Kirsch 1971, S. 53). Diese Sichtweise macht es möglich, Strukturen als kollektive Seite der Organisation zu betrachten. Organisationsstrukturen sind (wie die anderen Merkmale der Organisation auch) musterbildend für organisationale Entscheidungen, weil sie die Abläufe im Organisationsgeschehen beeinflussen: „Strukturen prägen Personen, sie kanalisieren, unterstützen oder behindern das Verhalten der Akteure" (Martin 1995, S.8), indem sie Einfluss auf das Selektieren, Ordnen und Verteilen von Informationen sowie die Ideen, Menschen, Lösungsvorschlägen, Handlungen etc. nehmen (vgl. Martin 1998, S. 32).

In einigen Organisationen wird den Entscheidungsverantwortlichen vorgegeben, wie sich beispielsweise die Auswahl eines/einer Bewerbers/-in vollziehen soll, indem die Organisation Kompetenz- und Stellenprofile sowie Ablaufbedingungen benennt. Damit haben sie ein vorgegebenes Entscheidungskorsett, in dem sie sich bewegen können. In anderen Organisationen mag sowohl die Entscheidung der Bewerberauswahl (wer wird ausgewählt?) als auch der Weg der Akteure/-innen (wie kommt es zu einer Entscheidung?) frei sein. Dieses Beispiel soll ver- 
deutlichen, dass die Organisationsstruktur zwar einerseits Entscheidungsfreiheit nehmen, aber anderseits auch den Organisationsmitgliedern Entscheidungsspielraum lassen kann. Die Organisation kann also mehr oder weniger Prozess- und Entscheidungsvorgaben machen. Je nach Struktur der Organisation sind Entscheidungswege deshalb mehr oder weniger gefestigt. Die Strukturen der Organisation haben eine gewisse musterbildende Richtung. Sie können aber nicht als eigenes institutionelles Instrument betrachtet werden, wohl aber als funktionales Subsystem der Organisation (vgl. Kirsch 1971, S. 53). Neben dieser Sichtweise existiert allerdings auch die Meinung, dass die Organisationsstruktur sehr wohl als institutionell zu betrachten sei und nicht lediglich funktionalen Charakter habe. Auch folgende Sichtweise bestätigt dies:

Die Organisation als Entscheidungssystem weist bestimmte interne Prozesse auf, die Entscheidungsstrukturen herausbilden und Entscheidungen produzieren ( $\mathrm{vgl}$. Cyert/March 1995, S. 23). Ausdruck von institutionellen Organisationsstrukturen sind u.a. administrative Prozesse, Handlungsvorgaben oder Hierarchien. Man denke dabei erneut an die Entscheidungswege zur Personalbe- 


\subsection{Merkmale von Organisationen}

schaffung: Von der Auswahlentscheidung, der Zustimmung des Betriebsrates bis zum ersten Arbeitstag ist das Handlungs- und Entscheidungsverhalten häufig festgelegt.

Institutionalisierte Prozesse befähigen die Mitglieder der Organisation, bei Auftreten eines Problems ein Ablaufprogramm ausführen zu können, das ihr Verhalten eindeutig und konfliktfrei steuert oder wenigstens einen Algorithmus zur Verfügung stellt, der aus den zur Wahl stehenden Lösungen die günstigste ermittelt (vgl. Schüßler 1993, S. 56). Der Ansatz von Scholl (2004) zielt in die gleiche Richtung, wonach Organisationsstrukturen als Grundlage institutionalisierter Prozesse dienen. Eine Organisationsstruktur bestehe in den von den Mitgliedern geteilten Grundannahmen, Werten und Normen der Organisation, von denen angenommen werde, dass sie die Gestaltung und Wahrnehmung von Prozeduren, Strategien und Strukturen beeinflussten (vgl. Scholl 2004, S. 532). Wenn also die am Organisationsgeschehen Teilnehmenden bereits Grundannahmen teilen, liegt es nahe, dass auch ihr Entscheidungsverhalten davon beeinflusst wird. Eine Annahme könnte sein, dass, je mehr die 


\subsection{Organisation und Entscheidungsmuster}

Mitglieder die gleichen Werte und Normen vertreten, sie auch in ihrem Entscheidungsverhalten kollektiv zusammenwirken und sich darin insgesamt ähneln.

Zusammenfassend können also die Strukturmerkmale der Organisation in bestimmte Entscheidungsmuster der Organisation münden, welche von den Merkmalen der Organisation bestimmt werden. Zweck, Geografie, Zeit, Organisationskultur und Organisationsstruktur sind Merkmale der Organisation, die die Handlungsbasis der Entscheider/-innen bilden.

\subsection{Organisation und Entscheidungsmuster}

Die Organisation und ihre Umwelt sind der Nährboden der Entscheidung. In diesem Umfeld gestalten sich die Entscheidungsabläufe und prägen das Bild der Organisation. Wie sich daraus spezielle Entscheidungsmuster ergeben, analysiert das folgende Kapitel. Wie kann nun die Organisation den Rahmen für die Entscheidung und deren Verlauf konkret bilden?

Im Mikrokosmos der Organisation und deren eigenen Umweltbedingungen finden die Mitglieder gewisse Vo- 


\subsection{Organisation und Entscheidungsmuster}

raussetzungen vor. Diese Voraussetzungen fließen in die Entscheidungsfindung ein und können in bestimmte Entscheidungsmuster münden, die aus dem Organisationsgebilde hervorgehen. Die Entscheidungsfindung ist ein Teil der Organisation, in der diese Verhaltensweisen institutionalisiert sein können (vgl. Martin 1995, S. 8). Entscheidungsmuster werden von verschiedenen Akteuren/innen in die Organisation integriert und (weiter)entwickelt. Sie sind das Entscheidungsgerüst mit verhaltenslenkendem Charakter, d.h., die Ausgestaltung der Organisationsmerkale prägt die Wege des kollektiven Entscheidungsverhaltens.

Entscheidungsmuster sind zu einem gewissen Grad institutionell normiert, weil die Prozesse organisiert und funktional gestaltet sind, z.B. durch Regeln und Richtlinien (siehe Kapitel 2.1). Daraus ergeben sich Trampelpfade, die sich aus formellen Strukturen und Prozessen ergeben. Diese müssen aber nicht immer eine institutionalisierte Wegbefestigung haben, sondern können sich auch informell herausbilden. Beispiel: In der Prozessbeschreibung "Die Vorgehensweise bei Mitarbeitereinstellung" ist beschrieben, dass der/die fachliche Vorgesetzte die finale Entscheidung trifft. Gelebte Praxis in der Organisation 
könnte aber sein, dass dem/der Geschäftsführer/-in aus Gewohnheit die finale Entscheidung überlassen wird oder der/die Fachvorgesetzte sich der Expertise eines/einer Recruiters/-in anschließt.

Das Beispiel zeigt, dass Institutionen Verhalten durch Richtlinien etc. normieren können, aber das Entscheidungsverhalten oft auch informell aufgeweicht werden kann. Damit entsteht eine Vermischung von formellen und informellen Strukturen und Prozessen. Für das Entscheidungsverhalten spielen formelle und informelle Kräfte der Organisation eine große Rolle; sie werden auch im weiteren Verlauf als entscheidender Wegweiser für die Entscheidungsfindung bei organisationalen Problemen dienen.

Organisationen sind verhaltensbildend für die Organisationsmitglieder, weil die Ausgestaltung der Organisation bereits ein Gerüst vorgibt. Nun soll der Entscheidungsprozess beschrieben werden, in den das Entscheidungsverhalten mündet. Damit soll im weiteren Verlauf der Arbeit die Trennung der Prozessschritte dem besseren Verständnis des Entscheidungsverhaltens dienen. 
entscheidungstheoretische Grundlagen

\subsection{Entscheidungsprozesse und entscheidungstheoretische Grundlagen}

Entscheidungsprozesse in der Organisation sind stark bestimmt von den Regeln, nach denen die Entscheidungsfindung abläuft (vgl. Cyert/March 1995, S. 95). Der zuvor angesprochene Organisationrahmen dient als Basis, um den Prozess der kollektiven Entscheidung im Weiteren zu verstehen (siehe Kapitel 2.1. und 2.2).

Um den Entscheidungsprozess in Organisationen zu beschreiben, verwenden viele Entscheidungsforscher/innen ausschließlich den Begriff der Entscheidungsphase, wobei auch Stadium, Stufe oder Schritte gebräuchlich sind (vgl. Schulz 1977, S. 22). Der Begriff der Phase impliziert eine Rangfolge, z.B. Phasen des Mondes oder Produktzyklusphasen; wobei zu untersuchen bleibt, ob es sich im Einzelnen um nacheinander ablaufende Phasen handelt. In diesem Abschnitt sollen die einzelnen Prozessphasen des Entscheidens näher beleuchtet werden.

Prozesse im Allgemeinen sind zunächst Mechanismen, die Aktivitäten und Denkmuster darstellen und strukturieren. Sie sind ein Ausdruck des Verhaltens von Organisationen (vgl. Martin 1995, S. 6). Anders ausgedrückt, sind 
entscheidungstheoretische Grundlagen

betriebliche Entscheidungsprozesse das Ergebnis einer Folge von innerbetrieblichen Verhaltensweisen oder, wie es Witte et. al. (1988) formulieren, des geistigen Produktionsprozesses. Erst durch die Abstrahierung des Forschungsgegenstandes „Entscheidung“ durch eine Differenzierung in einzelne Teilprozesse der Entscheidung ist es möglich, den Entscheidungsprozess als eine Folge sich wiederholender Entscheidungsprozesse aufzufassen und zu analysieren (vgl. Kirsch 1971, S. 52 f.). Untersuchungen von Entscheidungsprozessen fanden bereits in zahlreichen wissenschaftlichen Studien Beachtung. ${ }^{2}$ Doch durch die reine Vielfalt kann nicht darauf geschlossen werden, dass eine klare Abgrenzung der einzelnen Teilaktivitäten des Entscheidungsprozesses möglich ist, da diese oft stark ineinander greifen und verschwimmen: Entscheidungen sind vielschichtig und vieldeutig ( $\mathrm{vgl}$. Martin 1995, S. 22) und so auch ihre Prozesse.

\footnotetext{
${ }^{2}$ U.a. Janis 1982, Kirsch 1971.
} 


\section{Entscheidungsprozesses}

\subsubsection{Kriterien zur Darstellung des Entscheidungsprozesses}

Bevor die Prozesse des Entscheidungshandelns als (zeitlicher) Ablauf konkretisiert werden, ist eine Klarstellung einiger Analyseaspekte nötig. Um Entscheidungsprozesse zu beschreiben, sollen im Folgenden die Kriterien an die Darstellung, insbesondere im Hinblick auf die Komplexität und ihren zeitlichen Umfang, erläutert werden.

Die klassische Betrachtung des Prozesses in der Entscheidungsforschung basiert auf den Arbeiten von March und Simon (1958). Sie beschäftigten sich als Erste mit der Frage, nach welchen Prozessabläufen eine Auswahl hin zur Entscheidung getroffen wird. Sie definierten drei Phasen der Entscheidung: ${ }^{3}$

- Sammeln von Informationen

- Entwickeln eines Handlungsplanes

- Wahlphase

\footnotetext{
${ }^{3}$ So auch Rosenstiel/Nerdinger 2011, S. 347.
} 
2.3.1 Kriterien zur Darstellung des Entscheidungsprozesses

Ihre Forschung wurde im Laufe der Zeit weiter ausgebaut, und die Phasen wurden detaillierter. Die klassische Entscheidungsforschung konzentrierte sich auf die Betrachtung der Alternativenauswahl und dem letzten Entscheidungsakts, wobei die Entscheidungsforschung dabei eine retrospektive Sichtweise einnimmt (vgl. Martin 2001, S. 5 f.), d.h., die Entscheidung wurde erst nach ihrem Vollzug empirisch betrachtet. Eine Schwierigkeit, die vielen theoretischen Analysen immanent ist, scheint deshalb ihre „Rückwärtsgewandtheit" zu sein, d.h., Interpretation und Legitimierung finden auf der Basis von Vergangenheitsdaten statt. Diese Perspektive kann vor dem Hintergrund einer abgeschlossenen Entscheidung als finalen Elements des Prozesses dem Beobachter die Möglichkeit der rückwirkenden Betrachtung geben. Jedoch gehen einzelne Aspekte des Prozesses durch die nachträgliche Betrachtung, wie die Betrachtung des Entscheidungsgegenstandes, die Verhaltensweise im Entscheidungsprozess, die Behandlung nicht gewählter Entscheidungsalternativen etc., unter. Der Weg, der zur Entscheidung führt, gibt aber die Aufgabe auf, die Problemwahrnehmung stärker in den Vordergrund zu stellen als 
2.3.1 Kriterien zur Darstellung des Entscheidungsprozesses

die Lösungsorientierung, denn darin liegt das Forscherpotenzial, um Entscheidungsprozesse als Teilaktivitäten wahrzunehmen, zu beleuchten und ins organisatorische Handeln einzubinden. Martin (2001, S. 5) schlägt deshalb einen Perspektivenwechsel von der rückwärtsgerichteten Entscheidungstheorie zur Problemhandhabung vor. Auch diese Sichtweise erscheint einschränkend, wenn der Fokus lediglich auf die Lösungen bei der Entscheidung gerichtet ist. Vielmehr können Entscheidungen auch auf andere Entscheidungen bezogen sein, wie in Projekten, Entscheidungen über künftige Ziele, Verbesserungsentscheidungen etc.

Die Entscheidungsforschung basiert auf der Beschreibung und Erklärung verhaltenstheoretischer Forschung, d.h., die empirischen Grundlagen sind im Entscheidungsprozess durch eine Fokussierung auf bestimmte Variablen vereinfacht. Die Realität wird „im Reagenzglas“ dargestellt und empirisch erforscht, d.h., es wurden gewisse Konstellationen von Variablen analysiert, und in der jeweiligen Konstellation kommt es zu stabilen Entscheidungsprozessen. Die Realität von Laboruntersuchungen zum Entscheidungsverhalten ist gefiltert, wird 
2.3.1 Kriterien zur Darstellung des Entscheidungsprozesses

aber in vielen Wissenschaften zur Komplexitätsreduktion genutzt. Die Umwelt des Entscheidungsprozesses ist derartig komplex, dass der Kunstgriff der Ex-postAnalyse eher allgemeingültige Gesetzmäßigkeiten zutage fördert. Vieles spricht gegen ein solches Vorgehen. Trotzdem gibt es typische Muster, die einen Erkenntniswert haben, ohne sofort an handlungstheoretische Grenzen zu stoßen.

Um der Komplexität von Entscheidungsprozessen und ihrer Darstellung sowie Analyse gerecht zu werden, werden in der betriebswirtschaftlichen Wissenschaft mithilfe der mathematischen Einordnung durch Formeln o.Ä. Entscheidungsstrukturen abgebildet. Die Alternativenauswahl als logisch-analytische Rationalität erfährt eine Darstellbarkeit in Formeln (vgl. Gigerenzer/Gaissmaier 2006, S. 1), aber vernachlässigt dabei die weichen Elemente (wie Emotionen), die die Betriebswirtschaft von ihren Nachbardisziplinen, wie u.a. der Psychologie, flan- 
2.3.1 Kriterien zur Darstellung des Entscheidungsprozesses

kieren lässt. ${ }^{4}$ Das Erklärungspotenzial von Formeln, angewandt auf die entscheidungspraktische Wissenschaft, ist als gering einzustufen. Dies resultiert aus der Einsicht, dass es auch immer eine Reihe anderer Variablen gibt, die dadurch unberücksichtigt bleiben (vgl. Wossidlo 1975, S. 102 ff.). Um Entscheidungsprozesse adäquat abzubilden, muss aber auch der Anspruch bestehen, dass die Realität des Entscheidungsverhaltens in Organisationen wiedergegeben werden kann, und nicht, dass der Grad der Abstraktheit so realitätsfern ist, dass eine Darstellung nicht möglich ist. Weiterhin darf die Darstellung von Entscheidungsprozessen nicht zu stark vereinfacht dargestellt werden, damit sie in jede Entscheidungsschublade passt. Die Forschungssubstanz „Gruppenentscheidung“ stellt dabei aufgrund diverser Aufgaben, sozialer Effekte und Situationen eher eine Komplizierung als eine Erleichterung dar (vgl. Auer-Rizzi 1998, S. 161).

\footnotetext{
${ }^{4}$ Mit der Frage, wieviel Psychologie die Betriebswirtschaft in Bezug auf die Organisationsforschung braucht, hat sich u.a. Simon (1959, S. 82-108) beschäftigt. Auch die Psychologie hat ihre Grenzen in der Entscheidungsforschung. Diese beschreiben Nerdinger und Rosenstiel (2011, S. 367).
} 
2.3.1 Kriterien zur Darstellung des Entscheidungsprozesses

Diese Aspekte sind im Folgenden wichtig für die Bewertung der Qualität heuristischer Entscheidungen in Gruppen und deren ökologisch-rationaler Natur.

Die Beschreibung von Entscheidungsphasen ist in der Theorie umstritten, und ihre Vertreter/-innen argumentieren auch schlüssig: Zum einen sind die einzelnen Bestandteile des Entscheidungsprozesses nicht klar festgelegt; sie variieren oft zwischen drei und sechs Teilschritten. Zum anderen gehört das Beschreiben der Prozessaktivitäten eher zur normativen Entscheidungstheorie und wird dort als Handlungsmaxime gesehen, um den Entscheidungsprozess zu optimieren. Bei aller Kritik, die die Phasenmodelle erfahren, sowie der fragwürdigen Anwendbarkeit in der Realität soll diese Beschreibung dennoch erfolgen, um mit diesem Verständnis die Basis für weitere Überlegungen in Richtung ausgewählter Fehler in den kollektiven Entscheidungsprozessen von Gruppen zu bilden. Die Phasen sind durchaus nachvollziehbar und beschreiben die theoretische Prozesslogik immer ähnlich. 
Vor dem Hintergrund der drei genannten Analyseaspekte (Vermeidung von Retrospektive, mathematische Darstellung von Prozessen sowie Komplexitätsreduktion bei der Prozessdarstellung) soll also der Entscheidung als Auswahlphänomen auf die Schliche gekommen werden. Diese und andere Aspekte führten zu einem Umdenken in der Entscheidungsforschung, insbesondere im Hinblick auf die Rationalität einer Entscheidung. Simon prognostizierte bereits im Jahr 1965 (vgl. Simon 1965, S. 76) eine Revolution der Sichtweise auf die Entscheidungsrationalität. Der Rationalitätsaspekt soll deshalb ein besonderes Augenmerk in der vorliegenden Arbeit erhalten, und die Themen kollektives Entscheiden in Gruppen und Rationalität im Entscheidungsverhalten sollen kritisch betrachtet und miteinander verbunden werden.

\subsubsection{Der Entscheidungsprozess}

Das folgende Kapitel soll die Frage beantworten, wie Entscheidungen in Organisationen ablaufen und ob nach der Entscheidung der Entscheidungsprozess zu Ende ist. Der Prozess, bevor es zu einer Entscheidung kommt, ist die Wahl zwischen verschiedenen Alternativen, und es 
laufen einige Teilaktivitäten des Entscheidungsprozesses $a b$, die im Folgenden in sechs Teilschritte grob untergliedert werden: Problemwahrnehmung, kollektive Problemdefinition, Informationssuche, Auswahl, Umsetzung und Reflexion.

Der Entscheidungsprozess beginnt mit der Aufmerksamkeit für ein Problem. Auf eine Phase der Aufmerksamkeit durch die Wahrnehmung eines Problems folgen meist die aktiven Phasen der Einordnung und der Informationssuche, dann erfolgt die Suche nach einer Problemlösung durch das Sichten von Informationen und letztlich die Auswahl. Zwei weitere Aktivitäten sollen den Prozess abrunden: die Umsetzung und die Reflexion nach der Entscheidung. Im Folgenden werden die Phasen im Einzelnen beschrieben.

\section{Problemwahrnehmung}

Der Aufmerksamkeitsprozess zu Beginn des Entscheidungsverlaufes ist eine wichtige Phase, denn sie ist der 
Grundbaustein für weitere Überlegungen. ${ }^{5}$ Was ist die erste Teilaktivität in einem Entscheidungsprozess? Auch darüber scheint es keine einheitliche Meinung zu geben. Nach Meinung einiger Autoren/-innen sei der Entscheidungsanlass, d.h. das Vorliegen von Entscheidungsalternativen, bereits die erste Teilaktivität (vgl. Bender 1957, S. 76; Schulz 1977, S. 75), wobei sich die Problematik zeigt, dass dieses sich erstens schwer abgrenzen bzw. operationalisieren lässt und zweitens eine Diskrepanz zwischen dem Anlass und der tatsächlichen Entscheidung vorliegen kann, und drittens können die Alternativen unvollständig sein und erst im Laufe des Prozesses Gestalt annehmen. Der Anlass als erster „Entscheidungsgedanke" ist daher wenig überzeugend.

Für Simon (1959) beginnt der Entscheidungsprozess in einem frühen Stadium mit der aktiven Bearbeitung eines Problems. In der sogenannten editing phase werden die Entscheidungsalternativen in eine kognitiv vereinfachte Form gebracht, um eine weitere Verarbeitung erst zu er-

${ }^{5}$ Auch im Werbewirkungsmodell „Aida“ der Betriebswirtschaft (Attention, Interest, Desire, Action) beginnt der Kaufentscheidungsprozess mit der Aufmerksamkeit. Dieser Ablauf stellt im Wesentlichen gleichfalls ein Teilaktivitätenmodell dar, das zu einer Entscheidung führt. 
möglichen (vgl. Hinrichs 2011, S. 88). Andere Entscheidungsprozesse beginnen mit der kognitiven Einsicht, dass eine Entscheidung gefällt werden muss. Lyles (vgl. 1981, S. 64) geht davon aus, dass der kollektive Entscheidungsprozess beim Individuum beginnt. Gemeint ist nicht, dass ein/eine Einzelner/Einzelne den Entscheidungsprozess anstößt, sondern dass im Kopf der Organisationsmitglieder das Problem erkannt wird bzw. der Prozess des Wahrnehmens beginnt. Sie bezeichnet diese Phase als awareness oder auch incubation (a.a.O.).

Ein Entscheidungsanlass erscheint zunächst nur als eine Gegebenheit und erlangt Dynamik mit Eintritt in die Entscheiderwelt durch einen Stimulus. Die Entscheider/innen beschäftigen sich mit dem Entscheidungsanlass, sobald für sie ein Interesse daran besteht: Hier scheint der Entscheidungsprozess zu starten: Die Wahrnehmung des Problems beginnt.

In der Wahrnehmungsphase geht es zunächst darum, ein Verständnis des Problems zu entwickeln (vgl. SchäferPietig 1995, S. 13). Die Phase der Problemwahrnehmung umfasst eine Art Vorentscheidung darüber, ob die Fragestellung überhaupt untersuchungswürdig ist (vgl. Bender 1957, S. 76), denn bereits zu Beginn werden Lösungsal- 
ternativen durchgespielt. Die einzelnen Organisationsmitglieder erforschen die bestehenden Alternativen grob und bilden sich ein Urteil über die damit verbundenen Konsequenzen und Wahrscheinlichkeiten ihres Eintretens (vgl. Lindstädt 1997, S. 11). Hier zeigt sich die erste heuristische Vorgehensweise in der Entscheidungswelt. Wenn die Akteure/-innen das Problem erkennen, beginnt ein Analyseprozess, indem zunächst die eigentliche Fragestellung formuliert wird. Dieser Prozessteilschritt resultiert aus Wahrnehmungs- und Urteilsprozessen einzelner Gruppenmitglieder (vgl. Schäfer-Pietig 1995, S. 13). Bevor dies geschieht, vollzieht sich bei jedem/jeder Einzelnen ein individueller Wahrnehmungsablauf, der weniger einem kognitiven als einem stimulierenden Aufwand entspricht. Wahrnehmung ist ein geistiger Akt der sinnlichen Informationsregistrierung. Und auch visuelle und auditive Daten erreichen das Gehirn des Teilnehmenden und werden dort mit individuellen Normen wahrgenommen, verarbeitet und bewertet. Hierbei kann es im Laufe der Zeit zu einer kognitiven Musterbildung kommen (z.B. durch cognitive maps). Fehlende Informationen werden durch das eigene Wissens- und Wertesystem ergänzt. Die individuelle Wahrnehmung bildet den Ausgangspunkt 
im Gruppenentscheidungsprozess. In der vorliegenden Arbeit soll die Handlungsmotivation der Organisationsmitglieder den Beginn für des Entscheidungsprozesses markieren, d.h., es wird erkannt, dass es mindestens zwei Auswahloptionen oder eine Diskrepanz zwischen gegebenem und erwünschtem Zustand gibt (vgl. Jungermann 2009, S. 9).

\section{Kollektive Problemdefinition}

Ist der Entscheidungsprozess durch die Wahrnehmung des Problems angestoßen, überlegen die Organisationsmitglieder, was der Kern des Problems ist. Nach der individuellen Wahrnehmung folgt im nächsten Prozessschritt eine Vertiefung des Problems und die Öffnung des Individuums nach außen durch Kommunikation in der Gruppe (vgl. Wessler 2012, S. 113). Breite und Tiefe des Problems spielen eine wichtige Rolle für den weiteren Verlauf und dessen Ergebnis, denn damit tritt der Informationsgehalt, der sich um das Problem herum spinnt, zutage. Als nächstes Teilelement der Problemdefinition nennt Simon (1977) deshalb das Sammeln von Informationen. Je genauer die Problemdefinition, desto klarer die Überleitung zur Definition der Lösungssuche bzw. zur 
Suche nach Alternativen. Sind die Entscheidungsprobleme wohldefiniert, dann lässt sich ohne Schwierigkeiten anhand einer eindeutigen Definition der Ziele (wie der Lösungskriterien) angeben, ob eine Alternative tatsächlich eine zulässige Lösung des Problems darstellt (vgl. Schüßler 1993, S. 57). Daraus ergibt sich, dass die Entscheidungsaufgabe kein homogenes oder in sich geschlossenes Phänomen ist, sondern ein aus mehreren Elementen zusammengesetztes Gebilde darstellt (vgl. Schüßler 1993, S. 57).

Doch wie vollzieht sich die Problemdefinition im Kollektiv? In kollektiven Entscheidungsprozessen genügt es nicht, dass das Problem lediglich von Einzelnen wahrgenommen wird. Es muss auch vermittelt werden (vgl. Martin 1998, S. 42). In dieser Phase, die mit der Kommunikation der Organisationsmitglieder einhergeht, werden erste Bestimmungen des Problems vorgenommen, die sich im Prozessablauf weiter vertiefen, ergänzen, ausweiten oder ändern. Im Vorfeld der Gruppeninteraktion verändern sich Informationsstand und Bewertungen der Gruppenmitglieder im Hinblick auf Alternativen, Konsequenzen und Umweltzustände (vgl. Lindstädt 1997, S. 12). Kollektive Problemdefinition ist also eine verbale Operationali- 
sierung der Situation, um das Problem verständlich zu machen. Dies ist eine erste Weggabelung des Prozesses. Die Definition von Problemen bedeutet zugleich auch einen Ausschluss anderer Definitionen. Je nachdem, welche Erfahrungen die Entscheidungsträger/innen jeweils haben: Probleme werden von den Entscheidungsträgern/-innen heterogen definiert werden. Ein und derselbe Sachverhalt kann bei den verschiedenen Gruppenmitgliedern zu unterschiedlichen Bewertungen führen. Wie eine Problemdefinition im Kollektiv kognitiv repräsentiert wird, hängt u.a. davon ab, wie die Gruppe sozial interagiert (vgl. Schäfer-Pietig 1995, S. 13). Die kollektive Problemdefinition ist daher auch eine Möglichkeit der sozialen Informationsverarbeitung und der gemeinsamen Problemfestlegung. Mit der Problemspezifizierung wird zugleich auch eine Art Vorauswahl getroffen.

Entsprechend den der Organisation zur Verfügung stehenden Wahlalternativen wird das Problem von der Gruppe integriert (vgl. Cyert/March 1995, S. 98), d.h., indem die Aufgabenstellung eingegrenzt wird, beleuchtet die Gruppe zugleich die Machbarkeit mit den der Organisation zur Verfügung stehenden Mitteln. Mit dem einge- 
2.3.2 Der Entscheidungsprozess

grenzten Problem bzw. einer definierten Situation vor dem Hintergrund des Organisationsgeschehens geht es nun auf die gemeinsame Lösungssuche.

\section{Lösungssuche}

Entscheidungen können schnell und eindeutig getroffen werden, wenn die Lösungssuche durch Entscheidungskriterien erleichtert wird, die eine klare Definition haben. Dies ist dann der Fall, wenn Entscheidungsträger/-innen der Führungsebene zur Einordnung der Entscheidung genaue Richtlinien zur Lösungssuche vorgeben (vgl. Bender 1957, S. 79), d.h. die kollektive Willensinstanz der Organisation Entscheidungsprozesse definiert und institutionalisiert. Thompson (1967, S. 162) vertritt dabei die Meinung, dass diese Richtlinien dem einen Zweck der Unsicherheitsreduzierung dienten und in einen administrativen Prozess mündeten, zum Beispiel durch Arbeitsteilung, Standardisierung, Hierarchie, Kommunikation, Indoktrination (vgl. Schuler 2007, S. 57). Administrative Vorgaben formeller oder informeller Natur gibt es in nahezu jeder Organisation, entweder in schriftlicher oder mündlicher Form. Sie dienen der vereinfachten Lösungs- 
suche und sie schränken die Handlungsoptionen durch die Formalisierung der Organisation ein.

Doch nicht immer sind in dieser Phase die Alternativen, die zur Problemlösung zur Verfügung stehen, eindeutig identifizierbar. Zum einen, weil die Aufgabe oft nicht standardisiert werden kann (z.B. bei Kreativaufgaben), und zum anderen, weil die Institution keine Vorgaben für eine Aufgabe gemacht hat. Auch wenn die Handlungsoptionen und damit ggf. die Entscheidungswege normiert sind, stellt sich für die kollektive Entscheidungsfindung häufig die Frage, welche Lösungsalternativen es gibt. Tatsächlich sind die vermeintlichen Lösungen aber nur „Lösungshypothesen“, und es muss sich erst im weiteren Verlauf bzw. der Umsetzung zeigen, ob mit der gewählten Lösung die Probleme der Akteure/-innen wirklich beseitigt sind bzw. die Aufgaben gelöst werden konnten (vgl. Martin 1995, S. 66).

Die Voraussetzung für die Lösungssuche ist ein intellektuelles Erkennen der Lösungsalternativen (vgl. Simon 1977, S. 161). Anders als im Wahrnehmungsprozess (der eher die Stimulusareale anregt) sind hier auch die kognitiven Areale des Gehirns aktiv und verarbeiten die Informationen bewusst: Die Alternativen werden analy- 
siert und bewertet. Dieses aktive Tun geht nicht nur vom/von der Einzelnen aus, sondern verbindet die Individuen auf kollektiver Ebene durch Kommunikation. Es werden nun zwangsläufig die Alternativen und Bewertungen anderer mit einbezogen, die in einen erneuten Entscheidungsprozess einfließen (vgl. Wessler 2012, S. 113).

Entscheidungen, die getroffen werden, schließen auch in dieser Phase des Entscheidungsprozesses zwangsläufig andere Optionen aus, d.h., bereits während der ersten Betrachtung des Problems werden Optionen verworfen (vgl. Martin 1995, S. 4). Zum Beispiel kann das Problem „Der Vertriebsleiter kündigt und ein/eine Nachfolger/-in wird gesucht" von den Entscheidungsträgern/-innen dahingehend gelöst werden, dass die Suche einem Headhunter-Team übergeben wird. Aber die finale Entscheidung erfolgt organisationsseitig. Bereits durch diese Vorselektion der Entscheidung, indem sie also in andere Hände gegeben wird, werden Alternativen bei der Lösungssuche ausgeschlossen bzw. anders bewertet, da z.B. ein/eine Headhunter/-in andere Auswahlkriterien bei der Suche nach einem/einer Vertriebsleiter/-in anwendet als in einem internen Auswahlprozess. Damit sind sowohl 
2.3.2 Der Entscheidungsprozess

die kollektive Problemdefinition als auch die Lösungssuche vorselektive Teilaktivitäten, die den institutionellen Entscheidungshorizont limitieren können.

\section{Auswahl}

Die Frage nach dem finalen Akt der Entscheidung ist wichtig, aber sehr eng bemessen für einen wissenschaftlichen Anspruch. Im Gegensatz zur deskriptiven Entscheidungstheorie, in der der Entscheidungsprozess den gesamten Prozess der Willensbildung umfasst, bleibt die Entscheidung bei der normativen Entscheidungstheorie oft auf den Auswahlakt als finalen Entschluss beschränkt (vgl. Kirsch 1970, S. 70). Alle vorhergehenden und nachfolgenden Entscheidungsphasen sollten aber gleichfalls Raum in der Analyse des Entscheidungsablaufes einnehmen, um ein ganzheitliches Bild entstehen zu lassen und nicht nur die Wahl zwischen den Alternativen als Ceteris-paribus-Akt zu begreifen. Doch gerade auf die Auswahl legte die betriebswirtschaftliche Entscheidungsforschung in der Vergangenheit einen besonderen Fokus. Wie Joost (1975) bemerkt, besteht das Ergebnis von Arbeitsaufgaben in einem Entschluss. So auch Wossidlo (vgl. 1975, S. 102): Der Entschluss sei die Erforschung 
des letzten Entscheidungsaktes und damit die letzte Phase des Entscheidungsprozesses. Gleichwohl soll diese einengende Sichtweise nicht für die vorliegende Arbeit gelten, sondern durch den dynamischen Prozesscharakter soll Spielraum gelassen werden, denn die Entscheidung kann auch der Startschuss für einen neuen Entscheidungsprozess sein. Dies ist dann der Fall, wenn die Lösungssuche auf einem Folgeproblem basiert und eine Auswahl nicht die Teilaktivitäten Problemdefinition und Lösungssuche erfordern, weil ausreichend Informationen vorliegen und eine Entscheidergruppe die Alternativen bereits abgesteckt hat, sodass lediglich ein neuer Entschluss gefasst werden muss, weil das Entscheidungshandeln z.B. ein Folgeproblem ist (siehe Kapitel 1.2). Die Teilaktivitäten des Entscheidungsprozesses vermischen sich, starten wieder von Neuem oder werden beendet. Die Varianten der Prozessgestaltung sind deshalb sehr vielfältig und die Konstellationen. ${ }^{6}$ Es wäre zu kurz gegriffen, wenn lediglich der Entschluss im Forschungsfo-

\footnotetext{
${ }^{6}$ Um eine Betrachtung dieser Konstellationen hat sich u.a. Lyles (1981) bemüht.
} 
kus läge. ${ }^{7}$ Das Interesse an dem vermeintlich letzten $A b-$ schnitt des Auswahlprozesses ist vor dem Hintergrund betriebswirtschaftlicher Wertschöpfung verständlich. Denn die Entscheidung als solche stellt einen wesentlichen Beitrag zum Gesamterfolg dar und ist Ausdruck der Effizienz und des Ideenreichtums der Organisation.

Konkret ist die Auswahlentscheidung das Ergebnis und damit die Konsequenz vorgelagerter kognitiver und motivationaler Teilprozesse (vgl. Witte et.al. 1988, S. 9). Betrachtet wird dabei der Weg bis zur Auswahl durch das Kollektiv, an dessen Endprozess eine Einigung oder Nichteinigung steht oder der Prozess neu beginnt. Jede kollektive Auswahl wird eingeleitet, indem die Gruppenmitglieder gemäß eigener subjektiver Entscheidungskriterien hinsichtlich der Informationssuche und -verarbeitung in dem Auswahlprozess eine Vorauswahl treffen (vgl. Ahrens 1976, S. 29). Dies bildet das Fundament der kollektiven Auswahl.

${ }^{7}$ Nach Martin sollte diese Abstraktionsebene des organisatorischen Entscheidungsprozesses sowohl die Individualperspektive als auch das Organisationsverhalten integrieren (1995, S. 8). 
Wie gestaltet sich nun das Gesamtkonzept des Entscheidungsprozesses? Die unterschiedlichen kognitiven Bezugssysteme der Gruppenmitglieder mit ihren jeweiligen Entscheidungskriterien (vgl. Jungermann 2009, S. 13) können naturgemäß durch den kollektiven Austausch zu Interessenkonflikten führen. Den Aggregatzustand von Konfliktsituationen bestimmen die Gruppenmitglieder sowie die Organisationsstruktur. Um den Urteilsakt abschließen zu können, müssen die Gruppenmitglieder etwaige Konflikte zunächst lösen (vgl. Ahrens 1976, S. 27). Dieser Gedanke intendiert eine einheitliche Meinung; ein Konsens ist vonnöten. Konflikt, Kooperation und gemeinsame Abstimmung wirken dabei auf den Entscheidungsprozess mit einer starken Dynamik, die sich in der Diskussion durch Überzeugungsarbeit zeigt: Bestätigung, falls der Einzelne gleicher Meinung wie die Mehrheit ist; Zweifel, falls andere Meinungen stärker vertreten sind; Überraschung, wenn einem/einer klar wird, dass man mit seiner Meinung allein dasteht (vgl. Wessler 2012, S. 113).

Grundsätzlich stellt die Auswahl also nicht das Ende des Entscheidungsprozesses dar. Es wird lediglich eine Ent- 
scheidungsoption durch die Gruppe festgelegt, die umgesetzt und/oder bewertet wird (vgl. Jungermann 2009, S. 9). Letztere beide Teilaktivitäten (Umsetzung und Reflexion) werden nun betrachtet und in den Entscheidungsprozess integriert.

\section{Umsetzung}

Die Umsetzung der Entscheidung soll auch Eingang in den Entscheidungsprozess finden, da die Entscheidung als solche zwar den Beginn und das Ende der Aktivitäten darstellen kann, aber mit dem Entschluss zur Entscheidung der Entscheidungsprozess nicht abgeschlossen ist. Zu ihrem Erfolg kann die Umsetzung beitragen (wenn es zur Umsetzung kommt). Grundsätzlich kann davon ausgegangen werden, dass eine Entscheidung immer Handlungsimpulse nach sich zieht, die in Aktivitäten der Organisation münden (vgl. Ahrens 1976, S. 30). Damit ist die Umsetzung gewissermaßen das „To-do“ der Entscheidergruppe. Sie kann sich zum Teil deutlich anders darstellen als von den Entschlussgebenden festgelegt und beabsichtigt. Die Umsetzung trennt das Wollen vom Handeln (vgl. Sader 2000, S. 226). Auch hier kommt menschliches Verhalten zum Tragen, sodass Entschlüs- 
se missverstanden, umgedeutet, fehlinterpretiert oder sogar sabotiert werden können. Die Art der Umsetzung des Entschlusses ist dabei verzahnt mit dem organisationalen Geschehen (vgl. Martin 1998, S. 98).

\section{Reflexion}

In der Phase der Reflexion beurteilen die Mitglieder ihre gemeinsame Entscheidung. Gegenstand der Reflexion sind die Entscheidung, ihre Prozesswege, die Mitglieder und das Umfeld. Es handelt sich dabei um eine Ex-postBetrachtung. Erst, wenn der Prozess beendet ist, also entweder mit dem finalen Entschluss oder einem neuerlichen Sprung in eine der Kernentscheidungsphasen, kann die Reflexion beginnen. Unterstellt werden könnte hier natürlich, dass in jeder Entscheidungsphase reflektiert wird. Darauf könnte entgegnet werden, dass es bei der abstrakten Betrachtung der kollektiven Entscheidungsfindung sehr wohl eine Reflexion einzelner Phasen durch das Individuum gibt, jedoch die kollektive Reflexion erst im Nachgang des Prozesses erfolgen kann. Damit ist gemeint, dass organisationales Reflektieren erst dann greift, wenn Entscheidungen sichtbar werden. Was die- 
ser Ansicht zuträglich sein kann, ist z.B. der in vielen Organisationen genutzte Prozess des KVP (Kontinuierlicher Verbesserungsprozess) sowie des betrieblichen Verbesserungsmanagements. Dies sind zwei greifbare Werkzeuge kollektiven Entscheidungsverhaltens, die Teilaktivitäten retrospektiv reflektieren: Vergangene Prozesse werden „aufgerollt" und unter den Gesichtspunkten der Verbesserung institutionell beurteilt.

Wie zu Beginn des kollektiven Entscheidungsprozesses schließt sich auch hier ein zunächst individueller Wahrnehmungsprozess an. Das Individuum reflektiert zunächst allein und trägt dann die Reflexionsgedanken in die Gruppe. Ahrens (1976, S. 30) nennt diese Verankerung eine "subjektive Optimalitätseinschätzung" und meint damit die Messung durch das Individuums, das auftretende Diskrepanzen zwischen der Einstellungsund Verhaltensebene wahrnimmt und bewertet. Diese Reflexion unterliegt zunächst also individuellen Maßstäben. Wenn z.B. eine Meetingstruktur neu eingeführt wird, ist es für die einen eine lang ersehnte Regelung klarer Strukturen und für die anderen ist es überflüssige Bürokratie. Das kurze Beispiel zeigt, dass jede Entscheidung und ihre Umsetzung bei den Empfängern/-innen (die zu- 
2.3.2 Der Entscheidungsprozess

gleich Sender/-innen sein können) den individuellen Normen, Gefühlen, Einstellungen etc. unterliegen. Die Reflexion vollzieht sich zudem bewusst, aber häufig auch unbewusst. Reflexion der Gruppe hinterfragt organisationales Geschehen, woraus sich wieder neue Entscheidungsprozesse ergeben können, d.h., aus dem Reflexionsprozess kann sich wieder eine neue Teilaktivität ergeben. Beispiel: Es wurde eine Produktentscheidung getroffen, aber Kunden reklamieren die Ware, sodass die Entscheidungsteilnehmenden die Gründe hinterfragen müssen. Aus der Gruppenreflexion ergibt sich dann eine Überarbeitung des Produktes.

Der Reflexionsprozess geht (genauso wie die Problemwahrnehmung) von der individuellen zur kollektiven Ebene über, damit verbunden ist ein Abstimmungsprozess durch Kommunikation der Gruppenmitglieder bzw. anderer Prozessteilnehmender (wie Kollegen/-innen oder andere Personen aus der Organisationsumwelt) (vgl. Wessler 2012, S. 113). Kommunikation stellt das Bindeglied im kollektiven Entscheidungsprozess dar und ist die prozessbegleitende Komponente kognitiver Deutung, d.h., erst indem die Beteiligten über das Geschehene spre- 
chen, löst sich die Reflexion von der Individualebene und geht über zur kollektiven, gemeinsamen Reflexion.

McCall und Kaplan (1985, S. 31) sprechen nicht von Reflexion als einer Prozessaktivität, sondern von einer Interpretation des Ergebnisses. Sie greifen damit zu kurz, denn im Entscheidungsprozess vermögen die Mitglieder mehr als nur zu interpretieren. Reflexion bezieht sich auf den vollständigen Entscheidungsprozess und beleuchtet inn von allen Seiten und nicht lediglich das Ergebnis. Das Ergebnis ist ein entscheidender Teil des Entscheidungsprozesses, aber auch der Weg dahin kann beleuchtet werden. In der Reflexionsphase stellen sich verschiedene Fragen, z.B.: Wie ist die Entscheidung zustande gekommen, wer war beteiligt oder in welcher Umwelt fand das Geschehen statt? Die Ergebnisse können die Mitglieder zwar zufriedenstellen, obwohl der Prozess beschwerlich und lang war. Die Interpretation der Prozessphasen wird also ganzheitlich vorgenommen, sodass die Mitglieder ihre Schlüsse aus der kollektiven Reflexion ziehen können.

Die Ergebnisse der Gemeinschaftsreflexion können in den Entscheidungsprozess einbezogen werden, um diesen sodann anders gestalten zu können, z.B. durch eine 
andere Mitgliederzusammensetzung und -anzahl, bessere Themenabgrenzung (siehe 2.3.2 Problemdefinition), Komplexitätsreduktion bzw. -vertiefung durch Beschränkung bzw. Erweiterung der Auswahlalternativen (siehe 2.3.2 Lösungssuche). Damit kommt der Reflexion eine entscheidende Bedeutung im Entscheidungsprozess zu. Sie gibt inm eine qualitative Note, die in organisatorisches Lernen münden kann. ${ }^{8}$

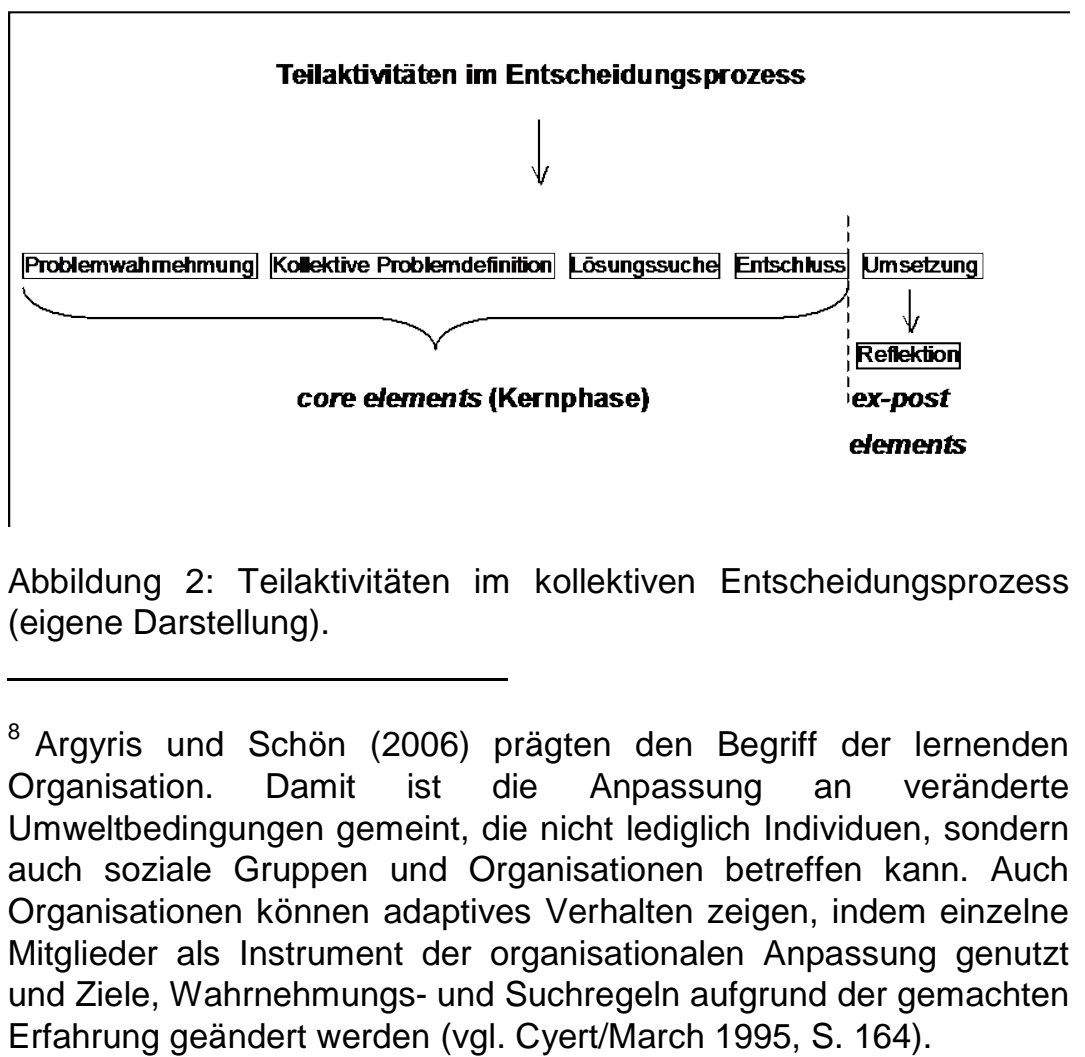


Häufig enden die Teilaktivitäten des Entscheidungsprozesses, wie in der Entscheidungsforschung üblich, mit dem Entschluss und ohne die Phasen Umsetzung und Reflexion zu betrachten. Die vier Teilaktivitäten kollektiver Entscheidung (Problemwahrnehmung, kollektive Problemdefinition, Lösungssuche und Entschluss) stellen die Kernprozesse kollektiver Entscheidungen dar (siehe Abbildung 2). Dass sich der Entscheidungsprozess Schritt für Schritt bis zur endgültigen Auswahl vollzieht, ist die übliche Sichtweise der Entscheidungstheorie (vgl. Martin 1995, S. 16), jedoch ist der Verlauf der Kernphasen im organisationalen Entscheidungshandeln unterschiedlich: Sie können teilweise zeitgleich verlaufen oder gar ausgelassen werden (vgl. Lyles 1981, Kirsch 1971). Entgegen dieser klassischen Betrachtung der Phasenrangfolge findet keine Aneinanderreihung von kettenähnlichen Prozessphasen statt. Entscheidungen können also unterschiedlich verlaufen und im Prozess wieder auf eine andere Teilaktivität wechseln (vgl. Martin 1998, S. 39). Die Übergänge sind aber mal mehr, mal weniger fließend, denn jeder Entscheidungsprozess verläuft anders (vgl. Kirsch 1971, S. 52). Lyles (1981) bezeichnet diesen 
Prozessablauf als Rückkopplung (vgl. auch Irle 1971, S. 52). Außerdem setzte sich neben der Erkenntnis der zeitlichen Überschneidung auch die personelle Überschneidung durch. Aufgrund wechselnder Personen ist der Entscheidungsprozess nicht personengebunden, und aus dieser Fluktuation ergibt sich eine weitere Instabilität. Dass der Prozess der Entscheidungsfindung eine Folge von sich zum Teil auch personell überschneidenden einzelnen Entscheidungsprozessen ist (vgl. Kirsch 1971, S. 52), macht den organisationalen Entscheidungsprozess zum kollektiven Entscheidungsprozess.

Die zwei Ex-post-Prozessphasen des Entscheidungsprozesses, Umsetzung und Reflexion, spalten sich von den vier Kernphasen der Teilaktivitäten ab (siehe Abbildung 2). Umsetzung und Reflexion haben im Prozess eine Sonderstellung. Der Entscheidungsprozess kann sich durchaus ohne Umsetzung und Reflexion vollziehen, aber beide Teilaktivitäten sind für die Qualität des Prozesses nicht unwichtig. Die Kernphasen markieren nicht die Vollständigkeit des Entscheidungsprozesses. Sie sind nicht abschließend oder getrennt von den Ex-postPhasen zu sehen. Wenn beispielsweise zum Ende eines Assessment-Centers eine Entscheidung für einen/eine 
Bewerber/-in getroffen wurde, durchläuft der Organisationprozess den Teilakt der Umsetzung mit der Einstellung des/der Kandidaten/-in sowie der Reflexion, ob er sich tatsächlich als geeignet herausstellt. Im Entscheidungsprozess sind Umsetzung und Reflexion notwenige Teilakte, ohne den der Prozess unvollständig erscheint. Sie sind nicht nur wichtige betriebswirtschaftliche Organisationselemente, sondern sogar hierfür entscheidend:

1. Organisatorisches Lernen, d.h. z.B.: Was kann beim nächsten Assessment-Center besser gemacht werden, wenn der Kandidat nicht passend war?

2. Wozu ist sonst eine Entscheidung nötig, wenn ihre Umsetzung außer Acht gelassen wird? Entscheidungsprozesse können nach der Umsetzung wieder neu beginnen bzw. danach reflektiert werden sollen.

3. Die Qualität jeder Teilaktivität kann durch Prozessfehler begrenzt sein (z.B. bei unzureichender Umsetzung durch schlechte Einarbeitung oder bei fehlender Reflexion, weshalb der Ablauf des nächsten AssessmentCenters wieder identisch wäre). Bei einer Darstellung des Entscheidungsprozesses ohne die Phasen Umsetzung und Reflexion wird der Organisation realitätsfern das 
„Verbesserungsmodul" genommen und damit werden ihr wichtige Erkenntnisse für künftiges Entscheidungsverhalten vorenthalten.

Nachdem die deskriptive Entscheidungstheorie mit ihren Veränderungen dargestellt, Begriffsbestimmungen (Entscheidung, Organisation) vorgenommen, Einflussvariablen des Entscheidungsverhaltens analysiert und Teilaktivitäten des Entscheidungsprozesses thematisiert wurden, wird im Folgenden ein ausgewähltes Modell der Entscheidung dargestellt und auf seinen Wert für kollektive Entscheidungsfindung analysiert. Das MülleimerModell von Cohen et al. (1972) soll das Entscheidungsverhalten in Organisationen beschreiben. Insbesondere durch die Feststellung im vorherigen Kapitel, dass Entscheidungsprozesse keinem normativen Ablauf folgen, soll an die Beschreibung von Organisationsverhalten angeknüpft werden. Das Mülleimer-Modell kann hierbei eine Antwort geben und im Vergleich zu anderen Modellen des rationalen Entscheidungsverhaltens auch komplexen und schlecht strukturierten Entscheidungsproblemen gerecht werden. 
2.3.2.1 Entscheidungen nach dem Mülleimer-Modell

\subsubsection{Entscheidungen nach dem \\ Mülleimer-Modell}

Das Mülleimer-Modell der Entscheidung von Cohen et al. (1972) ist ein Modell, um das Entscheidungsverhalten in Organisationen in mehrdeutigen, unklaren und komplexen Situationen zu beschreiben. Organisationen werden darin als organisierte Anarchien bezeichnet. Diese zeichnen sich durch unklare Ziele und Präferenzen (problematic preferences), fehlendes Technologiewissen (unclear technology) und wechselnde Organisationsteilnehmende (fluid participation) aus. Cohen et al. (1972, S. 2) beschreiben organisierte Anarchien so: „[...] this kind of organization is a collection of choices looking for problems, issues and feelings looking for decision situations."

Im Mülleimer-Modell wird der Prozess der Entscheidungsfindung als dynamisch dargestellt. Das Modell beschreibt das zufällige Zusammenspiel von vier Strömen (dies sind Teilnehmende, Lösungen, Probleme und Entscheidungsangelegenheiten), aus denen sich Situationen ergeben, die bestimmte Entscheidungen begünstigen. Das Modell beschreibt vier Strömungen, die durch die Organisation fließen und sich dann in einem „Mülleimer“ 
2.3.2.1 Entscheidungen nach dem Mülleimer-Modell

zusammenfinden (vgl. Cohen et al. 1972, S. 3). Diese Strömungen sind Teilnehmende, Probleme, Lösungen und Entscheidungsangelegenheiten. Sie sind unabhängige und dynamische Größen im Entscheidungsprozess. Erst eine bestimmte Kombination von Problemen, Lösungen, Teilnehmenden und Entscheidungsangelegenheiten führt zu einem Handeln der Organisationsteilnehmer (vgl. Kingdon 1995, S. 91). Diese Strömungen sind den Organisationsmitgliedern bekannt, jedoch nicht ihre Zusammensetzung, sodass Entscheidungen nicht das Resultat rationaler Analyse sind, sondern den zufälligen Zusammenfluss der vier Ströme darstellen. Es können zum Beispiel schon Lösungen für Probleme existieren, die noch gar nicht aufgetreten sind. Um dann zu Lösungen dieser Probleme zu werden, müssen die konkreten Probleme erst benannt werden.

Der „Mülleimer“ ist eine Sprachbild für dieses zufällige Zusammentreffen der Ströme. Die vier Strömungen sind zunächst voneinander unabhängig. Zum Beispiel sind Probleme und Entscheidungen klar voneinander getrennt und haben damit keine natürliche Abfolge, die auf Logik 
2.3.2.1 Entscheidungen nach dem Mülleimer-Modell

beruht; dadurch bekommt das Entscheidungsverhalten in Organisationen einen stochastischen Charakter.

Die vier Ströme im Einzelnen:

\section{Teilnehmende:}

Teilnehmende sind Organisationsmitglieder und können im Entscheidungsprozess von einer Entscheidung zur nächsten wechseln. Ihre Wechselmotivation, d.h., wie viel Zeit, Commitment und Aufmerksamkeit sie einer Entscheidung widmen, hängt von den Merkmalen und der Anzahl anderer, parallel laufender Prozesse ab.

In ihrem Modell haben Cohen et al. (1972) den Teilnehmenden ein festgelegtes Maß an Energie zugesprochen, d.h., der Teilnehmende stellt der Organisation im Allgemeinen potenzielle Energie zur Verfügung. Die Energie, die von den Teilnehmenden bemessen wird, ist limitiert. Ihre Lösungsenergie, also das Potenzial zur Lösung von Problemen, ermöglicht Entscheidungen, die zur Bearbeitung von Problemen geeignet sind. Cohen et al. definieren die Lösungsenergie so: „Each participant is characterized by a time series of energy available for organizational decision making. Thus, in each time period, each 
2.3.2.1 Entscheidungen nach dem Mülleimer-Modell

participant can provide some specified amount of potential energy to the organization" (1972, S. 3).

Teilnehmende können damit einerseits im Entscheidungsprozess als Ressourcen angesehen werden. Andererseits steht aber durch die Fluktuation ein hohes Potenzial an Energie zur Verfügung. Bei häufig wechselnden Teilnehmenden am Entscheidungsprozess entzieht der "Gehende" dem Prozess seine Energie (und damit auch sein Wissen), aber der „Kommende“ bringt seine potenzielle Energie wieder in den Energiestrom der anderen ein. Dem stehen natürliche Grenzen entgegen, wie die fachliche Qualifikation, ein möglicher Fachkräftemangel oder die unzureichende Motivation der Teilnehmenden, die einen Austausch der Lösungsenergie unter den Teilnehmenden nicht ermöglicht. Nicht jeder/jede kann jedes Problem lösen. Um den Energiestrom stabil zu halten, müssten Teilnehmende gefunden werden, die dem Entscheidungsprozess qualitativ gewachsen sind und auch den Willen mitbringen, sich einzubringen. Deshalb besitzen in einigen Strukturen die Teilnehmenden gleich viel Lösungsenergie, in anderen ist diese ungleich verteilt (vgl. Martin 1995, S. 63). Bereits in Kapitel 2.3.2 wurde der Entscheidungsprozess von den Teilnehmenden ab- 
2.3.2.1 Entscheidungen nach dem Mülleimer-Modell

gekoppelt, weil die Mitglieder von Organisationsgruppen regelmäßig wechseln und sich daraus ein Prozess personenunabhängiger Entscheidungen ergibt, der durch Mitglieder mit unterschiedlicher Lösungsenergie gekennzeichnet ist.

\section{Probleme:}

Probleme sind Anlässe aller Personen, die mit der Organisation im Zusammenhang stehen. Dies sind Probleme der Organisation selbst aber auch ihrer Umwelt. Es sind sowohl interne als auch externe Probleme, die in die Organisation hineingetragen werden. Probleme können sowohl kollektiver als auch individueller Natur sein: Jeder/jede einzelne Teilnehmende trägt seine/ihre Probleme mehr oder weniger in die Organisation hinein (z.B. private Probleme, Unzufriedenheit, Demotivation etc.) und genauso kann es Probleme geben, die vom Organisationskollektiv geteilt werden (z.B. Probleme, wie Tarifstreitigkeiten, Arbeitssicherheit, Betriebsübergänge etc.). Die Eigeninteressen der Teilnehmenden, wie seine/ihre Stelle zu sichern oder seine/ihre Abteilung zu vergrößern, können zu Lösungen führen, die nicht die Antwort auf ein Problem sind (vgl. Kingdon 1995, S. 90). 
2.3.2.1 Entscheidungen nach dem Mülleimer-Modell

Der Problembegriff wird folglich sehr weit gefasst und trägt damit der Realität Rechnung, da so gut wie jedes Problem die Energie der Organisation absorbieren bzw. auch Energie freisetzen kann. Jedoch könnte der Energie-Begriff in zwei unterschiedliche Begriffe unterschieden werden: Zum einen handelt es sich um Energie als die Ressource (mentale, zeitliche, Fertigkeiten), die zur Lösung verbraucht werden; zum anderen meint der Begriff kreative Lösungsenergie, die in der Auseinandersetzung mit einer anregenden Aufgabe entstehen kann (Generierung von Ideen).

\section{Lösungen:}

Lösungen sind die Produkte der Organisationsteilnehmenden. Während der Großteil der Lösungen klar an Probleme gekoppelt ist, unterstellen die Vertreter/-innen des Mülleimer-Modells, dass es Lösungen ohne Probleme geben könne. Dies ist z.B. in der Produktentwicklung von Innovationen der Fall, wenn ein Produkt entworfen wird, für das es noch keine Nachfrage gibt. In dem Modell werden Lösungen von Problemen abgekoppelt und es wird ihnen damit eine gewisse Eigendynamik gegeben. Es stellt sich vor allem die Frage, ob eine Entschei- 
2.3.2.1 Entscheidungen nach dem Mülleimer-Modell

dung immer zugleich auch eine Problemlösung bedeuten muss. Tatsächlich ist die vermeintliche Lösung oft nur eine "Lösungshypothese" (vgl. Martin 1995, S. 66), also eine Möglichkeit unter vielen, ein Problem zu lösen.

\section{Entscheidungsangelegenheiten:}

Entscheidungsangelegenheiten sind Situationen, in denen von den Organisationsteilnehmenden erwartet wird, eine Lösung zu finden. Diese Entscheidungsangelegenheiten gibt es zahlreich in Organisationen: z.B. Mitarbeiter/-innen einstellen, fördern, entlassen, Verantwortlichkeiten festlegen, Produkte verbessern. Entscheidungsangelegenheiten sind nicht notwendigerweise verknüpft mit Lösungen und Problemen.

\section{Entkopplung der Ströme:}

Das Mülleimer-Modell scheint der vorherigen Darstellung der Teilaktivitäten im kollektiven Entscheidungsprozess zunächst zu widersprechen: In Kapitel 2.3.2 (siehe Abbildung 2) ist die Rede von unabhängigen Teilaktivitäten, die zwar keine Reihenfolge darstellen, aber dennoch als natürliche Folge den Entschluss bzw. die Entscheidung haben. Nun geht aber das Mülleimer-Modell von einer 
2.3.2.1 Entscheidungen nach dem Mülleimer-Modell

völligen Unabhängigkeit der Strömungselemente aus, die auch noch ständig in Bewegung sind. Wie passt das zusammen? Sie stellen keinen Prozess dar. Die Strömungselemente sind Bausteine, die die Organisationsstruktur und die Umwelt vorgeben und die damit strukturgebunden bzw. passiv sind. Teilaktivitäten sind hingegen Taten, also aktive Aufgabenlösungen.

In der Darstellung der Teilaktivitäten in Kapitel 2.3.2 startet der Prozess mit dem Problem und kann mit der Entscheidung enden. Der Prozess wird umrahmt von den Kernphasen und ergänzt durch die Aktivitäten Umsetzung und Reflexion. Das Mülleimer-Modell geht hingegen davon aus, dass Teilnehmende, Lösungen, Probleme und Entscheidungsangelegenheiten wechselhaft und inkonsistent sind. Dies macht auch der Begriff Strom deutlich. Das Mülleimer-Modell widerspricht damit einer festen Reihenfolge der Entscheidungsverläufe und würfelt alle Elemente durcheinander. Dennoch ist die Unabhängigkeit der Ströme nur auf den ersten Blick evident, auf den zweiten Blick bedingen die vier Ströme einander, je nachdem, um welche „Konstellation“ es sich handelt. Das Zusammenfließen der Ströme wird durch die Organisationsstruktur kanalisiert, und es entstehen emergente Pro- 
2.3.2.1 Entscheidungen nach dem Mülleimer-Modell

zesse, die als „organisierte Anarchien“ bezeichnet werden. Der gewählte Begriff der Anarchie im MülleimerModell konnotiert bereits ein Abweichen von der Norm. Gemeint ist das Abweichen des rationalen Verhaltens vom tatsächlichen Organisationsgebaren. Bereits zu Beginn der vorliegenden Arbeit wurde die „begrenzte Rationalität im Organisationsverhalten" dargestellt (siehe Kapitel 2). Sie wird auch an dieser Stelle Grundlage sein. Jedoch gibt es Unterschiede: Im Gegensatz zu den begrenzt rationalen Theorien legt das Modelleimer-Modell seinen Fokus auf die Situation als zufällige Entscheidung, in der Timing und Glück eine Rolle spielen. Bei der Theorie der begrenzt rationalen Entscheidung spielt dies keine Rolle. Dort beginnen Entscheidungen mit einem Problem, das angemessen gelöst werden soll. Beim Mülleimer-Modell werden Entscheidungen zufällig gelöst, weil die Konstellation der Ströme dies so hergeben kann. Die Teilnehmenden und ihre Präferenzen springen innerhalb der Organisation von Entscheidungsangelegenheit $\mathrm{zu}$ Entscheidungsangelegenheit. Entscheidungen sind danach nicht das Ergebnis einer begrenzt rationalen Analyse, sondern unterliegen dem Einfluss des zufälligen 
2.3.2.1 Entscheidungen nach dem Mülleimer-Modell

Zusammenspiels der Ströme (vgl. Eisenhardt 1992, S. 27).

In organisierten Anarchien lassen sich Ansammlungen von „Mülleimern“ finden, in welche die verschiedenen Arten von Problemen und Lösungen hineingelegt werden (vgl. Sanders/Kianty 2006, S. 237). Welches Strömungselement in welchem Mülleimer landet, hängt u.a. von den Entscheidungsstilen ab. Cohen et al. (1972) extrahierten in ihren PC-Simulationen drei Entscheidungsstile. Sie geben einen Eindruck vom Verhalten in Organisation beim Umgang mit Entscheidungen. Sie sind jedoch nicht abschließend und können sich auch überschneiden.

\section{Entscheidungsstile:}

Problemlösung (,by resolution“)

Dieser Entscheidungsstil ist der am häufigsten unterstellte, insbesondere in der normativen Entscheidungsforschung. Nach der Abfolge der einzelnen Prozessschritte findet der Entscheidungsprozess sein Ende in der Entscheidung, die als Lösung des Problems gilt. Probleme werden nach einer Zeitspanne, in der an dem Problem 
2.3.2.1 Entscheidungen nach dem Mülleimer-Modell

gearbeitet wird, gelöst. Diese Zeitspanne kann variieren, je nach Anzahl der Probleme.

\section{Entscheidung durch Übersehen (,by oversight")}

Probleme können übersehen werden oder sie sind innerhalb des Problemkontextes ganz oder teilweise ausgeklammert, wenn zu dem Zeitpunkt noch andere Probleme behandelt werden. So können sogar schon Lösungen für Probleme gefunden werden, die noch gar nicht aufgetreten sind.

Beispiel: Die Auswahl von männlichen Bewerbern ist so lange problemlos möglich, wie in der Organisation das Thema der Frauenemanzipation noch nicht an das Problem „offene Stelle“ gekoppelt wird, obwohl es vielleicht bereits im Zusammenhang mit dem Problem der genderneutralen Bezahlung gesehen wird.

In diesen Entscheidungsstil könnte zugleich auch die Komplexitätsreduzierung als Problemlösemethode integriert werden, d.h., Informationen werden ausgeklammert bzw. ignoriert (dadurch reduziert sich die Komplexität) und die Lösungsfindung wird so erleichtert. Auch Kirsch (1988, S. 251) nennt Komplexitätsreduktion als Problemlösemethode und meint, dass angesichts hoher Kom- 
2.3.2.1 Entscheidungen nach dem Mülleimer-Modell

plexität und akuten Handlungsbedarfs dies durchaus eine sinnvolle Strategie sein könne, um Mängel und Störungen zu beseitigen.

\section{Entscheidung durch Flucht (,by flight")}

Auch Probleme können sich verflüchtigen. Das Mülleimer-Modell unterstellt, dass so lange versucht wird, das Problem zu lösen, bis es zu einer anderen Entscheidungsangelegenheit geflüchtet ist. Die Konnotation der Flucht könnte sprachlich eher durch Abwandern ersetzt werden, da dem Problem sonst eine Eigenaktivität („flüchten“) unterstellt werden kann, denn Probleme sind keine Wesen, sondern Sachverhalte. Richtig ist aber, dass Probleme von einer Entscheidungsangelegenheit in die nächste abwandern können. Gründe gibt es dafür zahlreiche. Insbesondere wenn für bestehende Probleme über längere Zeit keine Lösung gefunden wurde, so könnte eine bereits vorhandene Lösungsoption eine Umformulierung oder Definitionsänderung des Ursprungsproblems zur Folge haben, ohne dass dieses jedoch gelöst worden wäre (vgl. Sanders/Kianty 2006, S. 238).

Beispiel: Das Entscheidungsproblem „Integration ausländischer Mitarbeiter/-innen in den Betrieb“ wird zwar durch 
2.3.2.1 Entscheidungen nach dem Mülleimer-Modell

"diversity teams" und "Chancengleichheitsprojekte" gelöst werden können, dennoch kann es sich auch in Richtung ungleicher Bezahlung verlagern und damit in eine neue Entscheidungsangelegenheit verwandeln.

Organisierte Anarchien stellen ein Bild für die Dynamik des organisationalen Entscheidungsverhaltens dar und legen nicht den Fokus, auf Individuen als zentrale Entscheidungsträger/-innen. Je nach Ausgestaltung des "Strömungs-Mix“ in den Mülleimern bringt die Organisation als kollektiver Akteur mit ihren „Erfüllungsgehilfen“ die Entscheidung "hervor" (vgl. Neumer 2009, S. 20). Es ist also eher ein situativer Ansatz, der rationales Verhalten deshalb nicht in Betracht zieht, weil die Organisation und ihre internen Strukturen unvollkommen sind. Das Mülleimer-Modell legt den Fokus nicht auf individuelles Entscheidungsverhalten, sondern gibt der Organisation, ihren Grenzen und Bedingungen als Entscheidungsträger/innen Aufmerksamkeit.

Eine Mülleimer-Entscheidung zeichnet sich nicht dadurch aus, dass ihre Ergebnisse für "die Tonne“ sind, sondern eher auf den Zufall des Zusammentreffens gründen, statt einer steuerbaren (rationalen) Einflusswelt. Das Modell 
2.3.2.1 Entscheidungen nach dem Mülleimer-Modell

beschreibt Entscheidungen in Organisationen als mehr oder weniger zufällig (vgl. Jungermann et al. 2010, S. 400). Dieser Gedanke mag zunächst erstaunen, war doch zuvor die Rede von Organisationsstrukturen, die organisationales Verhalten lenken, denn die Merkmale der Organisation haben doch zuvor den Anschein erweckt, dass organisationales Entscheidungsverhalten nach Entscheidungsregeln und -mustern verläuft, die dynamische, aber konstante Prozesse sind. Die Merkmale der Organisation haben auch weiterhin verhaltenslenkenden Charakter, aber das Mülleimer-Modell stellt mehr den Zufall in den Vordergrund und ist damit geradezu das Gegenteil von rationalem Entscheidungsverhalten. Wie in Kapitel 2 dargestellt, geht die vorliegende Arbeit nicht von einem rationalen Entscheidungsverhalten in Organisationen aus. Die Organisationsmitglieder lassen sich zwar von strukturellen Merkmalen wie Verhaltensregeln leiten, aber ihr Verhalten ist nicht immer organisational intendiert. Das Mülleimer-Modell stellt auch infrage, ob die Teilnehmenden das Organisationsgeschehen überhaupt nachvollziehen können: „People also don't necessarily understand the organization of which they are a part: The left hand doesn't know what the right 
2.3.2.1 Entscheidungen nach dem Mülleimer-Modell

hand is doing" (Kingdon 1995, S. 3). Das MülleimerModell der Entscheidung soll im Folgenden den Blick auf ein dynamisches, ganzheitliches Entscheidungsverhalten innerhalb der Organisation schärfen, von dem Cohen et al. (1972) ausgehen.

Das auf den ersten Blick einfache und schlüssige Modell bezieht sich vornehmlich auf komplexe, mehrdeutige und schlecht strukturierte Entscheidungssituationen. Auch wenn keine Teilaktivitäten des Entscheidungsprozesses genannt werden, stellt das Modell zum einen die zuvor genannten Strömungselemente Teilnehmende, Lösungen, Probleme und Entscheidungsangelegenheiten dar und zum anderen die Entscheidungswege anhand von Entscheidungsstilen. Das Mülleimer-Modell ist für das Entscheidungsverhalten von besonderer Bedeutung, weil es endgültig mit regelbasiertem Verhalten aufräumt (vgl. Jungermann et al. 2010, S. 400). Mit dem Begriff „organisierte Anarchien" soll bereits die "Ungeordnetheit“ der Entscheidungsbausteine signalisiert, aber keinesfalls deren Beliebigkeit ausgedrückt werden. Für die vorliegende Arbeit ist das Mülleimer-Modell deshalb entscheidend, weil es aufzeigt, dass Prozesse ständig in Bewegung 
2.3.2.2 Entscheidungsdefekte von Gruppen im Mülleimer-Modell

sind und Probleme von Entscheidungen abkoppelt. Dies entspricht einem sehr nachvollziehbaren Gedanken: Nur weil eine Entscheidung getroffen wurde, muss nicht das Problem gelöst sein. Darin deutet sich auch bereits das Irrationale bzw. das Fehlerpotenzial an, um das es im Folgenden gehen soll.

\subsubsection{Entscheidungsdefekte \\ Gruppen im Mülleimer-Modell} von

Das Mülleimer-Modell betrachtet Entscheidungsdefekte auf struktureller Ebene anstatt auf Prozessebene. Cohen et al. (1972) richten den Fokus auf die Konstellationen ihrer vier Strömungen (vgl. Martin 1995, S. 63), denn die Strömungen des Mülleimer-Modells entfalten erst durch ihr Zusammenwirken einen Prozesscharakter. Grundsätzlich werden in dem Modell weder das kollektive Handeln noch die begrenzte Rationalität explizit genannt, sie sind aber dennoch ersichtlich:

1. Das Zusammenspiel der Strömungen kann erst durch kollektives Handeln möglich werden. Entschei- 
2.3.2.2 Entscheidungsdefekte von Gruppen im Mülleimer-Modell

dungen werden nicht isoliert von Einzelnen getroffen, sondern bedingen einander.

2. Soziale Entscheidungseffekte beruhen auf der sozialen Interaktion von Personen.

3. Aus der begrenzten Rationalität des Entscheidungsverhaltens und der Zufälligkeit des Zusammentreffens der Ströme können soziale Entscheidungseffekte, wie Defekte oder Heuristiken, resultieren.

Beim Mülleimer-Modell handelt es sich um eine ganzheitliche Betrachtung des organisationalen Entscheidungsverhaltens, die Gruppen damit einschließt. Außerdem klingen durch die Zufälligkeit allen organisationalen Handels Entscheidungsfehler im Verhalten an, werden aber nicht explizit benannt. In dem Modell wird die generelle Problemlöseaktivität der Organisationsteilnehmenden infrage gestellt und die Zufälligkeit des Entscheidungsverhaltens in den Entscheidungsstilen Flucht und Übersehen dargestellt. In jedem Entscheidungsstil und jeder Entscheidungskonstellation stecken deshalb potenzielle Entscheidungsdefekte und Heuristiken. Der Gedanke einer organisierten Anarchie impliziert bereits, dass Ent- 
2.3.2.2 Entscheidungsdefekte von Gruppen im Mülleimer-Modell

scheidungen einer gewissen Rationalität entbehren, sodass die Lösung zwar ein zufriedenstellendes Element darstellen kann, jedoch nicht das Optimum des Lösungshorizontes darstellen muss.

Beispiel: Fehlerhafte Einflüsse im Entscheidungsstil Problemlösung: Auch im Entscheidungsstil der Problemlösung kann es zu Entscheidungsfehlern innerhalb der Gruppen kommen. Zunächst wird davon ausgegangen werden: „Entscheidung getroffen = Problem gelöst.“ Dahinter steht häufig lediglich die Wahl einer Lösungsoption, da die am Entscheidungsprozess Teilnehmenden diesem Vorrang vor anderen Lösungsoptionen gegeben haben. Das heißt, nur weil eine Entscheidung getroffen wurde, ist dies nur eine Auswahl, aber muss nicht unbedingt die beste Lösung des Problems sein. Es kann z.B. sein, dass der Entscheidung das Commitment der Gruppenteilnehmenden fehlt, diese also nicht hinter dem Entschluss stehen.

Es sollte verdeutlicht werden, dass die Ideen des Mülleimer-Modells in die Überlegungen der Arbeit Eingang finden. Mit der begrenzten Rationalität des MülleimerModells und der Zufälligkeit menschlichen Handelns im 


\subsection{Zusammenfassung}

Hinterkopf sollen ausgewählte Entscheidungseffekte von Gruppen im nachfolgenden Kapitel 3 betrachtet werden. Hierbei soll das Hauptaugenmerk auf die Übertragung von der individuellen auf die kollektive Entscheidungsfindung gelegt werden.

\subsection{Zusammenfassung}

Die Entscheidungsforschung entwickelte sich im Laufe der Zeit zu einer deskriptiven Darstellung des Entscheidungsverhaltens in Organisationen. Sowohl rationales als auch begrenzt rationales Entscheidungsverhalten charakterisiert die Entwicklung der Entscheidungsforschung. Doch sind beide Konzepte wenig überzeugend im Hinblick auf alltägliches Entscheidungsverhalten von Organisationmitgliedern, weil nach dieser Sichtweise, die Organisationsmitglieder weiterhin einem intendiertrationalen Handeln unterworfen sind. Das heißt, die Individuen sind dazu angehalten (oder sie erlegen sich selbst diesen Anspruch auf), rational zu handeln. Tun sie dies nicht oder nur begrenzt, gilt ihr Handeln als defizitär. Schlüssiger erscheinen da die Erkenntnisse des Mülleimer-Modells von Cohen et al. (1972). Es beschreibt das 


\subsection{Zusammenfassung}

zufällige Zusammenwirken bestimmter Entscheidungskomponenten als Ströme. Die Erkenntnis aus diesem Modell ist, dass die Organisationsmitglieder weniger Einfluss auf die Entscheidungen haben, als sie denken. Der Zufall ist die wichtigste Entscheidungsgröße, die kaum beherrscht werden kann. Entscheidungsdefekte oder Heuristiken sind die Folge dieser Entscheidungen. Organisierte Anarchien spiegeln somit das Entscheidungsverhalten in Organisationen wider. Als Ausblick könnte die Verknüpfung von Heuristiken mit dem Mülleimer-Modell eine interessante Aufgabe für eine Studie darstellen.

Die Erkenntnisse der Entscheidungsforschung und des Mülleimer-Modells sind wichtig, um den nächsten Schritt hin zum heuristischen Entscheidungsverhalten zu tun. Das Konzept der begrenzten Rationalität war ein wichtiger Start, um sich von der Irrationalität rationaler Entscheidungen zu lösen. Um auch das Streben nach begrenzter Rationalität hinter sich zu lassen, sind weitere Schritte nötig. Hierzu soll im Folgenden das heuristische Verhalten in Organisationen konkretisiert werden. 


\section{Entscheidungsfindung von Gruppen}

Mehr Wissen und verfügbare Fähigkeiten, eine höhere Anzahl an Problemzugängen und die Möglichkeit der Eliminierung von fehlerhaften Schlussfolgerungen werden der Gruppe bei der Entscheidungsfindung attestiert (vgl. Auer-Rizzi 1998, S. 159). Dem effizienten Zusammenspiel von Gruppen steht aber eine Reihe von individual- und sozialpsychologischen Barrieren gegenüber. Dies können Phänomene, wie „Betriebsblindheit“, Gruppendruck oder der Einfluss von Vielrednern, sein (vgl. Rosenstiel/Nerdinger 2011, S. 344). Gruppenentscheidungen sind auch von vielfältigen Variablen abhängig, u.a. der Komplexität des Problems, dem zu erwartenden Zeitaufwand oder der Zielsetzung der Gruppenmitglieder. Bereits diese unvollständige Aufzählung lässt zahlreiche Kombinationen zu, die zu Hindernissen in der Entscheidungsfindung werden können.

Die Akteure der Organisation lösen Probleme, treffen Entscheidungen und agieren im Entscheidungssystem der Organisation. Dieses Phänomen wurde im ersten Teil 
3 Entscheidungsfindung von Gruppen

der Arbeit analysiert. Es standen die grundlegenden Kräfte der Organisation und ihrer Entscheidungsprozesse im Fokus. Im nächsten Kapitel wird nun weniger die betriebswirtschaftliche, sondern mehr die verhaltenspsychologische Sicht auf das Problemlöseverhalten betont. Denn das Problem des menschlichen Entscheidens, das nicht den rationalen Linien der Entscheidung zu folgen scheint, kann zu kognitiven Fehlern oder motivationalen Problemen führen (vgl. Gigerenzer/Gaissmaier 2006, S. 1). Dazu sollen die Teildisziplinen Betriebswirtschaft und Psychologie nicht gegenübergestellt werden, sondern sich ergänzen. Die Grenzen der einen Disziplin mögen die Anknüpfungspunkte zur anderen sein. Der Schwerpunkt soll im Weiteren auf den Dynamiken des Gruppenentscheidens, den Hindernissen und Heuristiken des Entscheidungsverhaltens liegen.

Wozu soll nun die Übertragung von individuellen Hindernissen auf die Gruppenebene dienen? Zum einen haben Gruppenentscheidungen eine hohe praktische Relevanz. Gruppenentscheidungen sind die gegenwärtige Form des Organisationsverhaltens. Die Verknüpfung von Entscheidungsprozessen mit Entscheidungsfehlern auf kollektiver 


\subsection{Erforschung von Gruppenentscheidungen}

Ebene scheint für Organisationen an Aktualität zu gewinnen, da in allen Branchen und Bereichen aus verschiedenen Gründen dazu übergegangen wird, Entscheidungen in Gruppen treffen zu lassen. Diesem Grundgedanken trägt die Arbeit Rechnung. Zum anderen kann die Übertragbarkeit individuellen Entscheidungsverhaltens einem besseren Verständnis der Gruppenebene dienen (vgl. Davis 1992, S. 33). Dabei geht es nicht um eine unkritische Verknüpfung von individuellen und kollektiven Verhaltensweisen, sondern soll einer Weiterentwicklung der Abgrenzung von Heuristiken auf Gruppenebene dienen, die bislang wenig wissenschaftliche Aufmerksamkeit erhalten haben. Außerdem leitet sich aus dem Wissen um individuelle Verhaltensweisen nicht automatisch eine Vorhersagbarkeit von kollektiven Organisationsentscheidungen ab. Schlussfolgerungen können lediglich aus den Erkenntnissen über die Eigenheiten der Entscheidungsleistung von Gruppen und ihren Prozessen ausgehen.

\subsection{Erforschung von Gruppenentscheidungen}

Die Erforschung des Phänomens Entscheidung hat eine lange Tradition und sie wurde in verschiedenen Teildis- 


\subsection{Erforschung von Gruppenentscheidungen}

ziplinen der Wissenschaft dargestellt und analysiert. In der Psychologie ist das Ziel der Entscheidungsforschung, Erkenntnisse zum Entscheidungsverhalten dahingehend zu finden, dass die Leistungsfähigkeit des „kognitiven Systems Mensch“ (vgl. Wossidlo 1975, S.100 ff.) erklärt werden kann. Hier klingt die psychologische Sichtweise der Entscheidungsforschung an und sie zielt nicht darauf $a b$, wie entschieden werden sollte, sondern wie Entscheidungen zustande kommen.

Dies geschieht sowohl allein als auch gemeinsam. Doch wie vollzieht sich eine Entscheidung im Kopf eines Menschen? Die kognitive Entscheidungsforschung der Psychologie konzentriert sich auf die Ebene des Individuums und bildet damit die Grundlage für ihre entscheidungstheoretischen Annahmen. Die Erforschung der Gruppenentscheidungen ist die kollektive Sichtweise der Organisationspsychologen/-innen. Sie untersuchen Verhaltenssituationen von Gruppen, wobei Konflikte und Informationsaustausch sowie Kommunikation eine zentrale Rolle spielen. Die organisationale Verhaltensforschung der Psychologie kann die Betrachtungen und Untersuchungen der Wirtschaftswissenschaft wertvoll ergänzen (vgl. Joost 1975, S. 1). 


\subsection{Erforschung von Gruppenentscheidungen}

Die Psychologie beschäftigt sich in vielfältiger Weise mit dem Phänomen der Entscheidungsfindung und, damit einhergehend, mit den Problemlösetechniken: empirische Versuche in Gruppen, Einstellung als Wegweiser des Problemlöseverhaltens bis hin zu Erfahrungslernen und Sozialisation. Die zahlreichen Forschungsfelder mögen einen Zusammenhang mit dem Problemlöseverhalten aufweisen, ob dem so ist, bleibt dahingestellt. Ein spezieller Punkt der psychologischen Entscheidungsforschung soll dabei herausgegriffen werden: das Problemlöseverhalten auf Gruppenebene vor dem Hintergrund von Entscheidungsdefekten und Heuristiken in der Organisation. Die Erkenntnisse dienen einem besseren Verständnis des Entscheidungsverhaltens in Organisationen und sollen für Fehler im Organisationsprozess sensibilisieren.

In der Entscheidungsforschung werden Entscheidungsfehler meist nur auf individueller Entscheidungsebene dargestellt. Doch allerorten wird das Thema Gruppenarbeit vor dem Hintergrund der Arbeitsplatzgestaltung, ergonomischer Überlegungen oder der Jobrotation etc. behandelt. Die Arbeitswelt überdenkt ihre herkömmliche Arbeitsumgebung, um Arbeit attraktiv zu halten. Deshalb scheint es angebracht zu sein, auch die kollektive Ent- 


\subsection{Erforschung von Gruppenentscheidungen}

scheidungsebene im Sinne des gemeinsamen Organisationsverhaltens zu erforschen, denn obwohl die Gruppenarbeit von jeder Disziplin hohe Beachtung erfährt, scheint die Verbindung zu kollektiven Entscheidungen weiterhin stiefmütterlich behandelt zu werden.

Um Entscheidungsfehler auf Gruppenebene zu analysieren und ggf. herzuleiten, sollen im Vorfeld folgende Analysefragen zur Orientierung gestellt werden. Vorgehensweise und Forschungsfragen sollen damit schematisiert werden:

- Sind individuelle Entscheidungsprozesse auf kollektives Entscheidungsverhalten übertragbar? Können individuelle verhaltenstheoretische Modelle um die kollektive Ebene ergänzt werden?

- Was ermöglicht eine Übertragung von der individuellen auf die kollektive Entscheidungsebene?

- Welche Eigenschaften hat der kollektive Entscheidungsprozess von Gruppen?

- Welche Rolle spielen die Qualität und Komplexität der Entscheidung?

- Welche Entscheidungsfehler von Gruppen können gruppenperspektivisches Entscheidungsverhalten angemessen darstellen? 
3.1 Erforschung von Gruppenentscheidungen

- Welche Untersuchungen gibt es, die die Gruppenebene mit einbeziehen?

Ein Erklärungsansatz für den Fokus früherer Untersuchungen auf die individuelle Entscheidungsforschung ist, dass Entscheidungen weiterhin nur dem TopManagement der Organisation vorbehalten sind, d.h., Einzelentscheider/-innen, wie Unternehmensinhaber/innen, Geschäftsführer/-innen, Bereichsverantwortliche, Standortmanager/-innen etc., geben die "Marschrichtung“ der Organisation vor und treffen die Entscheidung für die Organisation. Diese Art der Organisationsbetrachtung ist weder zeitgemäß noch tragfähig. Selbst wenn es den/die patriarchalischen/matriarchalische Entscheider/-in alten Stils noch geben sollte, verlässt auch er/sie sich nicht nur auf sein/ihr eigenes Urteil, sondern berät sich, kommuniziert, stimmt sich ab oder greift auf externe Hilfe zurück. Die „Legende“ des/der Einzelentscheiders/-in mag zwar die Fokussierung der Forschung darauf erklären, sie erscheint jedoch nicht angemessen. Als Vorteile von Gruppenentscheidungen nennen Eisenführ und Weber (2010, S. 312) dafür folgende Aspekte:

- Mehr Ideen generieren 
3.1 Erforschung von Gruppenentscheidungen

- Mehr Wissen über Fakten und Zusammenhänge

- Ausgleich fehlerhafter Einschätzungen einzelner Mitglieder

Bereits die unterschiedlichen Hierarchieebenen machen eine Gruppenabstimmung nötig. Hinzu können hierarchiebezogene Gewichtungen kommen: Sind in einem Projektteam vier Vorgesetzte, von denen zwei eine ranghöhere Position einnehmen, hat die Stimme (unabhängig von einem Abstimmungsprozess) der ranghöheren Gruppenmitglieder oft mehr Gewicht. Dies ist z.T. sogar dann der Fall, wenn es sich um heterogene (abteilungsübergreifende) interdisziplinäre Kollektive handelt. Auch kann das Entscheidungsverhalten von ranghöheren Gruppenmitgliedern von einer kollektiven Note geprägt sein. Ein weiteres Beispiel: Der Organisationsverantwortliche des Bereiches Controlling stimmt sich mit seinen Mitarbeitern/-innen ab, weil sein Wissen in Bezug auf einen spezialisierten Teilbereich seiner Abteilung limitiert ist. Außerdem berät er sich mit der Geschäftsleitung über die weitere Vorgehensweise von Projekt $A$ bezüglich der Budgeterweiterung. Erst nach der Abstimmung ist es inm möglich, eine Entscheidung zu treffen, die aber auf einer 
3.1 Erforschung von Gruppenentscheidungen

gemeinsamen Entscheidungsfindung beruht.

Die Organisationspolitik setzt vermehrt auf Gruppenarbeit. Dabei wird also unterstellt, dass Gruppen über mehr Problem- und Wissensressourcen verfügen als Einzelpersonen (vgl. Reimer et al. 2007, S. 8). Umstritten ist hingegen, ob ein Ausgleich von Entscheidungsfehlern des Individuums durch die Gruppe erfolgt. Trotz der Vorteile, die Mehrpersonenentscheidungen mit sich bringen, bezieht sich dies nach Meinung einiger Autoren/-innen nicht auf den Ausgleich der Defekte (vgl. u.a. Auer-Rizzi 1998, S. 161). Das hängt u.a. damit zusammen, dass die Mitglieder oft mehreren Entscheidungseffekten unterworfen sind. In der psychologischen Forschung zum Problemlöse- und Entscheidungsverhalten von Gruppen wird ein möglichst vollständiger Informationsaustausch nahegelegt, um das Wissenspotenzial mehrerer Mitglieder auszuschöpfen (vgl. Reimer et al. 2007, S. 8). Doch einige Forscher/-innen geben zu bedenken, dass Gruppen ihr Wissenspotenzial in der Regel nicht ausschöpften (u.a. Stasser/Titus 1985, Hinsz et. al. 1997, S. 43-64). Auer-Rizzi (1998, S. 160) meint, dass etliche Eigenheiten des Gruppenprozesses, z.B. Konformitätsdruck und Po- 
3.1 Erforschung von Gruppenentscheidungen

larisierung, zu Prozessverlusten im Gruppenverhalten führten, die blockierten und Vorteile verhinderten. Die Besonderheiten von Gruppen tragen dazu bei, und auch die vielfältigen Rahmenbedingungen haben Einfluss auf die Gruppenentscheidung. Soziometrische Struktur, Machtstruktur, Kommunikationsstruktur, Rollenstruktur, Führung, Konformität, Bezugsgruppen etc. sind Quellen für das Entscheidungsverhalten und für Entscheidungsfehler (vgl. Neumer 2009, S. 12). Auch nach Martin (vgl. 1998, S. 3) kann der soziale Rahmen die Entscheidungsfindung der Gruppe negativ beeinflussen. Andere Autoren/-innen nehmen beides an: dass das soziale Verhalten der Gruppe sich sowohl negativ als auch positiv auf die Qualität der Entscheidung auswirken könne (vgl. Drewes et al. 2011, S. 228). Oft wird unterstellt, dass bei der Qualität von Gruppenentscheidungen auch oft eine Verminderung der Entscheidungsverzerrungen durch einen Ausgleich der Gruppe erfolgt, weil die Gruppe über große Ressourcen verfügen soll, und zwar in Bezug auf Wissen, Fähigkeiten, eine höhere Anzahl von Problemzugängen und die Möglichkeit zur Eliminierung von fehlerhaften Schlussfolgerungen (vgl. Auer-Rizzi 1998, S. 159). 
3.1.1 Abgrenzung von Einzel- und Gruppenentscheidungen

Jedoch kann konstatiert werden, dass dies eine sehr allgemeine Sicht ist, die aber im Konkreten zu untersuchen ist. Vor diesem Hintergrund soll aber im Folgenden das Gruppenverhalten in Abgrenzung vom individuellen Entscheidungsverhalten dargestellt werden. Anschließend sollen sich ausgewählte Entscheidungsdefekte explizit auf den Gruppenentscheidungsprozess beziehen, um exemplarisch die Verzerrung des Entscheidungsverhaltens auf kollektiver Ebene darzustellen. Komplexität und Qualität sowie Besonderheiten des kollektiven Akteurs sind dabei flankierende Themen, um das Investigationsfeld „Entscheidungen in Gruppen“ abzurunden.

\subsubsection{Abgrenzung von Einzel- und Gruppenentscheidungen}

Einfluss auf die Entscheidungsfindung der Gruppe haben neben individuellen Faktoren auch soziale Mechanismen (vgl. Schäfer-Pietig 1995, S. 16). Zunächst scheinen aber kollektive Problemlösetechniken einen Widerspruch in sich zu tragen. Probleme können zwar gemeinsam gelöst werden, aber die Techniken scheinen nur dem Individu- 
3.1.1 Abgrenzung von Einzel- und Gruppenentscheidungen

um vorbehalten. Auch die verhaltenspsychologische Entscheidungsforschung legt einen Schwerpunkt auf das individuelle Entscheidungsverhalten. Dieses wurde hinreichend intensiv erforscht, und daraus ergaben sich valide Befunde (vgl. Auer-Rizzi 1998, S. 159). Entscheidungen werden aber, wie bereits erwähnt, selten von einer zentralen Machtinstanz getroffen, sondern von Entscheidungskollektiven in Form von Gruppen, Ausschüssen oder Arbeitskreisen. Alleinentscheidender bedienen sich des Organisationskollektivs zur Entscheidungsvorbereitung, zur Informationsgewinnung oder als Vorschlagsgrundlage. Auf allen Entscheiderplattformen bewegen sich Organisationsteilnehmende mit unterschiedlichsten Merkmalen (Hierarchieebene, Bildung, Persönlichkeit etc.) zum Zwecke der gegenseitigen Abstimmung, Beratung und letztlich der Entscheidungsfindung. Oft sind die Gruppen ${ }^{9}$ in Organisationen formalisiert und

9 Unter einer Gruppe soll eine Ansammlung von Individuen verstanden werden. In der sozialen Gruppe interagieren die Gruppenmitglieder für einen längeren Zeitraum miteinander (vgl. Lindstädt 1997, S. 1). Eine andere gängige sozialpsychologische Definition ist die von Tajfel und Turner (1986, S. 24). Sie definieren eine „Gruppe als eine Ansammlung von Individuen, die sich selber als Mitglieder derselben sozialen Kategorie wahrnehmen, die ein 
3.1.1 Abgrenzung von Einzel- und Gruppenentscheidungen

standardisiert. Es sind in der Regel mehrere Personen an Problemlösung und Entscheidungsfindung beteiligt, um unterschiedliche Sichtweisen, Lebensvorstellungen und Wissensbestände einzubringen und sich auch auf Prozessebene (Handlungsschritte) abzustimmen (vgl. Boos 1996, S. 9). Da Probleme in der Organisation meist mehrere Organisationseinheiten, Abteilungen oder Spezialgebiete betreffen, soll von einer kollektiven Entscheidungsfindung in Gruppen ausgegangen werden. Auch wenn eine Einzelperson die Verantwortung für die Entscheidung trägt und diese vielleicht sogar endgültig trifft, ist der Prozess der Entscheidungsfindung kollektiver Natur und deshalb die gegenseitige Abstimmung oft unerlässlich (siehe u.a. Beispiel in Kapitel 3.1).

Letztlich beherbergt diese Ansicht den Gedanken, dass Organisationsmitgliedern in leitenden Positionen die Aufgabe der Gruppenführung, Moderation oder inhaltlichen Lenkung zukommt (u.a. Prozessgestaltung und -lenkung,

gewisses $\mathrm{Maß}$ an emotionaler Bindung an diese Kategorie aufweisen und die über einen gewissen sozialen Konsens verfügen“. Die Bezeichnungen für Kollektive Gemeinschaften, wie Teams, Gruppen oder Gremien, werden aber im Folgenden synonym verwendet. 
3.1.1 Abgrenzung von Einzel- und Gruppenentscheidungen

Vorgaben definieren, Mitarbeiter/-innen führen etc.). Diese Ansicht nimmt der Organisation damit dennoch nicht die hierarchisch-finale Entscheidungsgewalt oder gibt ihr etwa ein sozialistisches Gesicht („alle entscheiden mit“), sondern sie spiegelt sich die Vorbereitung und die Beteiligung unterschiedlicher Organisationsakteure an der Entscheidung wider. Mitarbeiter/-innen werden in Entscheidungen der Organisation einbezogen, und durch dieses Involvement können motivationale Dynamiken wie Commitment gegenüber der Gruppe o.Ä. entstehen und kooperativ organisationale Ziele verfolgt werden (vgl. Drewes 2013, S. 145). In vielen Organisationen wird es als gewünschtes Führungsverhalten gesehen, die Mitarbeiter/-innen mit „ins Boot“ zu holen, z.B. durch die organisationalen Vorgaben aus Führungsleitlinien.

Gruppen sind aufgrund ihrer Vernetzung innerhalb der Organisation schwer abgrenzbar. Sie neigen dazu, durch vielerlei Einflüsse zu variieren. Selbst Laboruntersuchungen vermögen nicht alle Variablen konstant zu halten. Die Modellierung von Gruppenentscheidungen in Untersuchungen kann dabei eine beachtliche Komplexität er- 
Gruppenentscheidungen

reichen (vgl. Sauermann 2012, S. 81). Außerdem gibt es in der Organisationsrealität keine Kollektiventscheidung als einstimmige Gruppenmeinung, wie es im politischen Sozialismus idealiter der Fall wäre. Auch wenn die Ziele der Organisation äußerlich eine Einigkeit darstellen, bleiben sie abstrakt und sind nicht explizit als Präferenzen oder Meinungen bei den Organisationsakteuren verankert. Im Gegenteil, Präferenzen konkurrieren miteinander bzw. stehen in einem Konflikt zueinander (vgl. Eisenhardt 1992, S. 27). Gruppenentscheidungen sind also die Summe divergierender Meinungen und deshalb schwer vorhersagbar, da jedes Individuum innerhalb der Gruppe unterschiedliche Präferenzen und Bezugssysteme hat. Sauermann (2012, S. 81 ff.) hat sich mit der Frage beschäftigt, wie sich individuelle von kollektiven Entscheidungen unterscheiden (drei Erklärungsansätze). Mit dieser Frage beschäftigt sich auch die Sozialpsychologie im Rahmen des Gefangenendilemmas. Die Ergebnisse finden eine Erklärung u.a. im Diskontinuitätseffekt, den Insko und Schopler (1972) beschrieben haben, die auch den Unterschied zwischen individuellem und kollektivem Handeln darstellen. Der Diskontinuitätseffekt besagt, 
3.1.1 Abgrenzung von Einzel- und Gruppenentscheidungen

dass das Individuum weniger Vertrauen in eine Fremdgruppe hat und sich nicht mit ihr identifizieren kann, weil es aus der Eigengruppe mehr Unterstützung erfährt. Im Rahmen ihrer Untersuchung wurden Entscheidungen von Einzelpersonen mit den Entscheidungen von Gruppen verglichen. Dabei fiel das Verhalten der Gruppenentscheidung im Vergleich zur Einzelentscheidung insgesamt kompetitiver aus, weil das Eigeninteresse durch den „social support" der Gruppe gestärkt wird (vgl. Insko/Schopler 1972, S. 135).

Dafür kann es drei Erklärungsansätze geben (vgl. Sauermann 2012, S. 819 f.):

1) Die Mitgliedschaft in Gruppen erschwert die Zuschreibung individueller Verantwortung: Das Individuum ist in der Gruppe schwerer identifizierbar, als wenn es allein handeln würde. Deshalb ist der/die Einzelne in der Gruppe weniger geneigt, egoistisch zu handeln. Die Verantwortung des einzelnen Organisationsmitgliedes wird bei der kollektiven Entscheidung an die Gruppe abgegeben. 
3.1.1 Abgrenzung von Einzel- und Gruppenentscheidungen

2) Die Gruppendynamik bestärkt die einzelnen Akteure/innen in ihrem Entscheidungsverhalten: Die Gruppenmitglieder bestärken sich gegenseitig darin, dass ihr Entscheidungsverhalten in Ordnung ist, das möglicherweise sogar eine Verletzung von Organisationsnormen darstellen kann. Die (normverletzende) Entscheidung wird dann durch die Gruppe eher legitimiert, als eine Einzelentscheidung dies tun würde. Beispiel: Die Gruppe fährt zu einer Tagung und überschreitet dabei das vorgegebene Reisekostenlimit. Da aber alle Gruppenmitglieder mit dieser Abweichung von der Organisationsrichtlinie konform gehen, fühlt sich der/die Einzelne bestätigt und hat durch die Gruppenlegitimation kein Störgefühl (wie Unrechtsbewusstsein o.Ä.).

3) Egoistisches Verhalten gegenüber der Umwelt: Akteure/-innen in Gruppen senken ihre Kooperationsbereitschaft gegenüber der Umwelt, weil die Meinung vor- 
3.1.1 Abgrenzung von Einzel- und Gruppenentscheidungen

herrscht, sich gegenüber anderen Gruppen abgrenzen zu müssen (verstärktes Konkurrenzdenken/Wettbewerb). ${ }^{10}$

Diese Erklärungsbeispiele verdeutlichen die Grundlagen des Entscheidungsverhaltens in der Gruppe. Der wesentliche Unterschied zwischen individuellem und kollektivem Verhalten liegt im sozialen Prozess (vgl. Schäfer-Pietig 1995, S. 12), d.h., die Entscheidung findet in einem interaktiven Kontext statt. Deshalb stellt sich die Frage, ob es neben aller Individualität des Entscheidens auch einen gemeinsamen Organisationshorizont in Organisationen gibt, der Gruppen beim Entscheiden leitet?

10 Siehe auch die Theorie der sozialen Identität von Turner und Tajfel (1986), in der (Inter-)Gruppenprozesse analysiert werden. Auch Festingers (1954) Theorie des sozialen Vergleichs kann als Basistheorie zur Erklärung sozialer Prozesse durch Vergleiche mit anderen Leistungen oder als Meinung zur Verifizierung der eigenen Sichtweise in Ermangelung objektiver Normen herangezogen werden. 
3.1.2 Besonderheiten des kollektiven Entscheidungsakteurs

\subsubsection{Besonderheiten des kollektiven Entscheidungsakteurs}

Bei der Abgrenzung des individuellen vom kollektiven Entscheidungsverhalten ging es um die Unterschiede, die das Entscheidungshandeln in der Organisation ausmachen, und darum, dass Entscheidungen in Organisationen meist kollektiver Natur sind. Diese kollektive Natur des Entscheidungshandelns soll im Folgenden auf Züge eines einheitlichen Organisationswillens der Organisation als des kollektiven Entscheidungsakteurs untersucht werden. Die Organisation kann beim kollektiven Entscheidungsverhalten ein entscheidender Einflussfaktor sein. Denn nicht nur die externen Umweltfaktoren, wie etwa der Arbeitsmarkt, spielen eine Rolle, sondern auch das "interne" Handeln des kollektiven Akteurs, also der Organisationsgemeinschaft an sich. Aber was ist gemeint, wenn es heißt, die Organisation sei ein kollektiver Akteur, wenn doch die Individuen oder die Teilnehmenden der Gruppe die ganze Arbeit machen?

Wenn die Organisation als kollektiver Akteur arbeitet, handelt sie natürlich nicht selbst, sondern ist durch Organe und ihre Teilnehmenden vertreten. Organschaftliches 
3.1.2 Besonderheiten des kollektiven Entscheidungsakteurs

Handeln ist bereits im HGB, GmbHG und AktG verankert (z.B. durch Vorstand, Aufsichtsrat, Gesellschafterversammlung etc.). Organisationale Prozesse zeichnen sich dadurch aus, dass das Entscheidungsproblem von einer übergeordneten Instanz (Geschäftsführung, Vorstand, Vorgesetzte etc.) an die Entscheidungsträger/ -innen innerhalb der Organisation delegiert wird (vgl. Lindstädt 1997, S. 9). Den Begriff Instanz benutzt Lindstädt in seiner Untersuchung über Gruppenentscheidungsprozesse, um das organisationale Handeln zu betonen. Er geht davon aus, dass Entscheidungsprobleme der Gruppe stets Metaentscheidungsprozesse sind, d.h., das Problem „läuft" bei der Instanz auf, und diese erhält dann die Gelegenheit, Steuerungsvariablen in ihrem Sinne festzulegen, sodass eine optimale Problemlösung möglich wäre. Die Organisation als kollektiver Akteur delegiert die Entscheidung dann an die Organisationsmitglieder. Delegieren findet immer dann statt, wenn es sich um nicht routinemäßige Aufgaben, hier schlecht strukturierte Probleme handelt (vgl. Schüßler 1993, S. 1). Doch auch bei schlecht strukturierten Problemen bzw. nicht routinemäßigen Aufgaben kann die Organisation mitagie- 
3.1.2 Besonderheiten des kollektiven Entscheidungsakteurs

ren. Das Delegieren ist die Arbeitsteilung der Organisation, für die unterschiedliche Fachkenntnisse gebraucht werden, um eine Auswahl vor dem Hintergrund des organisationalen Anforderungs- bzw. Anspruchsniveaus zu treffen.

Neben den zahlreichen einzelnen Organisationsmitgliedern kommt der Eigenständigkeit des einheitlichen Makroakteurs (der Organisation) eine besondere Bedeutung bei der Entscheidung zu. Diese unterliegt einer besonderen Art von Rationalität ${ }^{11}$, weil die Organisation ihrerseits einen Einfluss auf die Akteure ausübt (vgl. Sanders/Kianty 2006, S. 173). Dieser Gedanke meint aber nicht, dass unter den Akteuren ein einheitlicher Organisationswille vorgefunden werden könnte, der von einer einheitlichen Meinungsbildung geprägt wäre. Es handelt sich eher um einen zentralen Einfluss des Organisationskollektivs und nicht um eine Einheitsmeinung der Organi-

11 Um die Rationalität von Entscheidungen zu unterstützen, antwortet die Entscheidungstheorie mit der formalen Organisation, d.h. durch Reduktion von Unsicherheit und Komplexität mittels Arbeitsteilung, Standardisierung, Hierarchie, Kommunikation, Indoktrination etc. (vgl. Schuler 2007, S. 57). 
3.1.2 Besonderheiten des kollektiven Entscheidungsakteurs

sation. Letzteres ist selten der Fall, da die Entscheidungsfindung durch multiple Faktoren beeinflusst wird.

Die Träger/-innen betrieblicher Entscheidungen sind Gruppen oder Einzelpersonen (vgl. Schüßler 1993, S. 13), und auch wenn die Darstellung des kollektiven Entscheidungsverhaltens unabhängig von der individuellen verhaltenspsychologischen Handlung sein soll, stellt sie trotzdem die unabdingbare Basis für die Gruppenbetrachtung dar. Der individuelle Einfluss auf die Gestaltung der Organisation und ihre Entscheidungsprozesse ist die Basis für das Entscheidungsgebäude der Organisation. Und auch Gruppenhandlungen gehen zunächst von individuellen Gedanken Einzelner aus. Diese verzahnen sich erst im zweiten Schritt durch Interaktion.

Es stellt sich aber dazu die Frage, ob das kollektive Entscheidungsverhalten nicht eine gewisse Eigenständigkeit innehat. Denn werden einzelne Organisationsmitglieder ausgewechselt, bleibt trotzdem so etwas wie ein "Spirit" erhalten. Hierbei könnte es sich um einen allgemeinen Gestaltungswillen handeln, der der Organisation immanent ist, auch wenn sich die Umweltbedingungen ändern. Handlungen und Entscheidungen in Organisationen sind 
3.1.2 Besonderheiten des kollektiven

\section{Entscheidungsakteurs}

in erster Linie Gegenstand bewusster Koordination. Dieses System ist in der Organisationsgruppe als unveränderlich anzusehen. Ein Wechsel der Organisationsteilnehmenden führt nicht zu einer Umwälzung des Organisationssystems. Die verschiedenen Organisationsteilnehmenden als Entscheidungsträger/-innen stellen nicht nur die Beschäftigten der Organisation dar, sondern es zählen auch Kunden, Lieferanten und Aktionäre, die sogenannten Share- und Stakeholder, dazu. ${ }^{12}$ Die Organisation hat also weiterhin Bestand, trotz des Ein- und Ausscheidens von Organisationsteilnehmenden, und verändert auch nicht ihren organisationseigenen Charakter. Als Beispiele können die Organisationsstruktur angeführt werden, so, wie der langfristige und schwer änderbare Charakter von Organisationskulturen ${ }^{13}$ (vgl. Martin 1995, S. 60). Handlungen werden von der Organisation

12 Cyert und March (1995) unterscheiden in ihrer verhaltenswissenschaftlichen Theorie der Unternehmung zwischen Beschäftigten und Teilnehmenden. Letztere nehmen eher passiv am Organisationsbetrieb teil - wobei dies zu diskutieren wäre.

${ }^{13}$ Eine Besonderheit stellen hierbei Gründerpersönlichkeiten dar, die der Organisation bisweilen ein strukturgebendes Gesicht hinterlassen, das noch Jahrzehnte später Handlungsmaxime ist, wie z.B. im Falle des Apple-Gründers Steve Jobs. 
3.1.2 Besonderheiten des kollektiven Entscheidungsakteurs

unter dem Gesichtspunkt des Organisationszwecks betrachtet (siehe Kapitel 2.1). Der kollektive Akteur, die Organisation, leitet daraus seine Entscheidungen ab. Diese Sichtweise hat unzweifelhaft ein indoktrinierendes Element: Die Organisation gibt vor, und die Organisationsteilnehmenden werden in ihrem Handeln von der Organisation beeinflusst (z.B. durch Schulungen oder gezielte Personalauswahl). Die Organisation stellt Entscheidungsprämissen auf, welche die Komplexität der Auswahl im Entscheidungshandeln reduzieren sollen. Hierbei handelt es sich u.a. um die Standardisierung von Prozessen. Indem wie beispielsweise Hierarchien in der Organisation gestaltet sind, wird das Handeln der Organisationsteilnehmenden begrenzt.

Die Mitglieder werden von der Organisation in ihren Entscheidungen geführt, wobei sich dieser Einfluss eher indirekt zeigt und nicht als aktive Beeinflussung in Erscheinung tritt. In vielen Unternehmen gibt es beispielsweise ungeschriebene Einstellungsprämissen, wie etwa: „Regionale Bewerber/-innen passen besser zu uns, weil sie nicht so oft den Arbeitgeber wechseln“ - und danach werden dann die Bewerber/ 
3.1.2 Besonderheiten des kollektiven Entscheidungsakteurs

-innen priorisiert. Dies kann u.a. aus gemachten Erfahrungen herrühren, die die Organisation mit regionalen Bewerbern/-innen gemacht hat.

In dieser Betrachtung sollen die Entscheidungen des kollektiven Akteurs eher unpersönlicher Natur sein und nicht auf einzelne Organisationsteilnehmende oder Gruppen zurückgeführt werden, sondern einen losgelösten Leitungshorizont charakterisieren. Es handelt sich in der Theorie (der Entscheidung) um eine abstrakte Betrachtung eines einheitlichen Handlers. Und wenn die Organisationsteilnehmenden sich mit der Organisation identifizieren, berücksichtigen sie unter Umständen in stärkerem Maße die Ziele des kollektiven Akteurs. Die Organisationsteilnehmenden stellen quasi die Erfüllungsgehilfen/-innen des kollektiven Akteurs dar. Gruppen sind dabei die autorisierten Gremien des Handelns (vgl. Martin 1995, S. 7). Es handelt sich also bei der Gruppe um ein autorisiertes Gremium, das im Sinne des Makroakteurs der Organisation agieren kann und ein absichtsgeleitetes Entscheidungsverhalten an den Tag legen soll. Gruppen entscheiden in einem kleineren Entscheidungsmilieu als 
3.1.2 Besonderheiten des kollektiven Entscheidungsakteurs

die Organisation in ihrer Gesamtheit. Betrachtet man die Handlungsweise der Organisation als Einheit, so sind die Organisationsziele der kognitive Ariadnefaden. Er wird durch die Arbeitsteilung der Organisation ausgelegt.

Die Organisation als Makroakteur zu behandeln, gründet sich darauf, dass einzelne Organisationsteilnehmende vornehmlich ihre eigenen Ziele verfolgen, aber sich trotzdem ein kollektiver Wille herausbilden kann (vgl. Martin 1995, S. 6). Aber nicht nur organisationale Faktoren beeinflussen das Entscheidungsverhalten aktiv, sondern auch intra- und interindividuelle Faktoren der Organisationsteilnehmenden, wie Machteinflüsse, Trittbrettfahren etc. Intra- und intersoziale Prozesse sind das Bindeglied zwischen der individuellen und der kollektiven Perspektive. Meinungsbildung oder (Vor-)Entscheidungen basieren in der Organisation zunächst auf individuellem Auswahlverhalten, aber das Entscheidungshandeln ist in den meisten Organisationen eher kollektiver Natur. Damit ist gemeint, dass das Individuum zunächst für sich allein eine Entscheidung trifft. Um aber als finale Entscheidung in ein Entscheidungshandeln einzugehen, bedarf es der 
3.1.2 Besonderheiten des kollektiven Entscheidungsakteurs

Abstimmung mit anderen Organisationsteilnehmenden. Erst dann erhält der organisatorische Gestaltungswille Ausdruck. Die Verlinkung von individuellem und kollektivem Willen vollzieht sich dabei durch Kommunikation. Je nach Aggregatzustand ist die Kommunikation eher fließend oder eher zähflüssig. Eine fließende Kommunikation ermöglicht den Organisationsteilnehmenden einen guten Austausch von Informationen und ein fließendes Entscheidungshandeln mit hoher Kompetenz oder gar Qualität.

Die organisationale kollektive Entscheidung ist idealiter rational und verfolgt konsistente Ziele. Grundsätzlich ist das Musterbild des rationalen Organisationsgebarens ein hehres Ziel. Aber von politischen Parteien als kollektiven Entscheidungsträgern wird beispielsweise davon ausgegangen, dass ihre getroffenen Entscheidungen eindeutig und frei von Präferenz- und Vorteilsdenken seien. Hierbei handelt es sich um einen theoretischen und idealistischen Anspruch, der aber nicht den Kern des Entscheidungshandelns in Organisationen trifft. 
3.1.2 Besonderheiten des kollektiven Entscheidungsakteurs

In der verhaltenswissenschaftlichen Theorie der Unternehmung von Cyert und March (1995, S. 114 ff.) haben die Koalitionen von Individuen keine konsistenten Ziele und ihr Handeln ist nicht rational. Sie werden als „adaptiv rational" beschrieben, da auch die Organisation als Ganzes nur über begrenzte kognitive Informationsverarbeitungskapazitäten verfügt kann und damit auch ihre Handelnden nicht allwissend sind. Das Individuum stellt zwar den zentralen Referenzpunkt im Entscheidungshandeln dar, jedoch ist die Idee des kollektiven Akteurs der Organisation als einheitlich und eindeutig Handelnder eine andere. Die Organisation reduziert durch ihre quasi unbewegliche und zeitkonstante Struktur die Unsicherheit von Individuen. Sie macht gewisse Vorgaben, die den Entscheidern/-innen bereits eine Richtung vorgibt. Dieses Richtungsdenken ist auch als adaptiv rational einzustufen, denn Individuen „rütteln“ zwar zeitweise an diesem Konzept, indem sie die Organisation mit ihren Ideen und Taten beeinflussen, aber das Grundkonzept bleibt prinzipiell in jeder Organisation erhalten und wird von der Organisation vorgegeben, nicht von den einzelnen Individuen. Es ist, wenn die Organisation bereits lange besteht, 
3.1.2 Besonderheiten des kollektiven Entscheidungsakteurs

über Jahre gewachsen und gefestigt worden. Es zeigt sich beispielsweise auch in Mergers \& AcquisitionsProzessen, dass Organisationsstrukturen nur schwer zu verändern sind.

Der Anpassungsdruck durch Globalisierung und Internationalisierung, dem Organisationen ausgesetzt sind, zwingt sie, Schritt zu halten und sich anzupassen. Bei einem solchen Eingriff in die Organisation handelt es sich um Marktentscheidungen, die den Organisationszweck betreffen, aber nicht in die Grundfesten der Organisationsstruktur eingreifen. Aber auch „junge“ Organisationen, wie Start-up-Unternehmen haben bereits zu Beginn eine Organisationsstruktur. Diese ist zwar noch flexibler und weniger ausgestaltet, aber durchaus vorhanden. So, wie Organisationsstrukturen (siehe Kapitel 2.1) der Verhaltensregulierung dienen (vgl. Martin 1998, S. 5), bilden sie zugleich auch eine mehr oder minder stabile Basis für die Entscheidung des kollektiven Akteurs. Sie können von den Organisationsteilnehmenden sogar in Teilen beeinflusst werden, sind aber ein relativ konstantes Merkmal der Organisation. In Bezug auf die Organisation als einheitlichen Entscheider stellen die Organisationsstrukturen 


\subsubsection{Komplexe Entscheidungen}

das Handlungskorsett dar (siehe Kapitel 2.1). Die Eigenheiten der Organisationsstruktur bilden das Charakteristikum der Organisation ab. Danach agiert sie als Ganzes mittels ihrer Erfüllungsgehilfen, der Individuen.

Komplexität ist ein Merkmal von Entscheidungen in Gruppen, von dem immer ausgegangen wird, dass es die Entscheidung erschwere und Prozessfehler begünstige. Doch was bedeutet es für die Gruppe, eine komplexe Entscheidung treffen zu müssen? Dies wird im Folgenden anhand des Entscheidungsmerkmals der Komplexität erläutert und soll im Anschluss eine Betrachtungsgröße zur Bewertung der Entscheidungsqualität von Gruppen sein.

\subsubsection{Komplexe Entscheidungen}

Grundlage für weitere Überlegungen sind also komplexe Gruppenentscheidungen, die wichtige und schwierige Probleme in Organisationen betreffen können. Komplexe Entscheidungen können z.B. im Unterschied zu Routineentscheidungen dazu führen, dass der Zuwachs von neuen Ideen in der Gruppe im Laufe des Entscheidungs- 
prozesses abnimmt, je komplexer die Entscheidung ist (vgl. Boos 1996, S. 104). Komplexe Entscheidungssituationen haben eine große Anzahl an Variablen (als quantitatives Hindernis), die abhängig voneinander wirken können (als qualitatives Hindernis) und die dadurch die Vorhersagbarkeit (vgl. Höpfner 1997, S. 199) bzw. sogar die Problemlösung erschweren oder sogar unmöglich machen (vgl. Schüßler 1993, S. 104). Die Merkmale der Organisation (siehe Kapitel 2.1) sind das „Handlungsumfeld“ der Entscheider/-innen und sie beeinflussen zugleich die Entscheidungssituation, d.h. die entscheidungsbezogenen Merkmale der Organisation (wie Zweck, Geografie, Zeit, Organisationskultur und struktur). Sie wirken als Nährboden des Entscheidungsverhaltens. Komplex bedeutet insbesondere, dass die Problemstruktur differenziert und vernetzt ist (vgl. Boos 1996, S. 103). Organisationen sind immer einer Vielzahl von Einflüssen ausgesetzt, und die Organisationsmitglieder sehen sich mit der Herausforderung konfrontiert, mit diesen umzugehen und sie bis zur Entscheidungsreife zu bewerten, zu urteilen und anschließend die Entscheidung umzusetzen. 
3.1.3 Komplexe Entscheidungen

Folgende Bedingungen sollen bei der weiteren Vorgehensweise berücksichtigt werden (vgl. Borcherding/Schäfer 1976, S. 51):

1. Die Entscheidung ist kompliziert und von Bedeutung.

2. Die Entscheidung wird in einer Organisation getroffen.

3. Es sind mehrere Personen an der Entscheidung beteiligt.

4. Die Entscheidungsalternativen müssen von Entscheidern/-innen erst erarbeitet werden. ${ }^{14}$

Mit einer komplexen Entscheidung als Handlungssituation ist es auch eine Herausforderung, die Qualität von Entscheidungen in Gruppen abzubilden. Der Erfolg der Entscheidung ist aber von theoretischem und praktischem Interesse und stellt die Leistung der Gruppe dar. Deshalb stellt sich im Folgenden die Frage, wie komplexe Entscheidungen in Gruppen bewertet werden können.

${ }^{14}$ Die Entscheidungssituation muss vor allem zunächst interpretiert werden. Bei komplexen Entscheidungen handelt es sich nicht um eine Standardsituation, sondern dabei bedarf es der Kreativität der Gruppe. Hinrichs (2011, S. 28) bezeichnet diese Art komplexer Entscheidungen als konstruktive Entscheidungen mit hohem kognitiven Aufwand. 


\subsubsection{Bewertung von Entscheidungen}

Entscheidungen sind für die Organisation ein wichtiges Steuerungselement, sowohl strategisch als auch operativ. Doch Messbarkeit, Kriterienabgrenzung zur Einordnung der Qualität und Reliabilität sind bei komplexen Entscheidungen mit vielfältigen Variablen schwer operationalisierbar. ${ }^{15}$ Denn wer mag schon die Frage zur Zufriedenheit beantworten, welche Entscheidung als eine gute angesehen werden kann. Sanders und Kianty (2006, S. 185) folgen der betriebswirtschaftlichen Definition von Entscheidungsqualität und verstehen darunter, dass mit den zur Verfügung stehenden Mitteln ein maximaler Gewinn erwirtschaftet bzw. ein Ziel mit minimalen Kosten erreicht wird.

Doch dieser Rational-choice-Ansatz beantwortet nicht die subjektive Einschätzung: Was der/die eine als Entscheidung von hoher Qualität betrachtet, mag in den Augen des/der anderen defizitär erscheinen. Daran schließen sich weitere schwer zu lösende Fragen an, wie bei-

${ }^{15}$ Zum Beispiel meint im juristischen Sprachgebrauch Qualität „die Lieferung mittlerer Art und Güte" (§ 243 II BGB). Sie ist damit keine Entscheidungsleistung von 100\% der möglichen Leistung. 


\subsubsection{Bewertung von Entscheidungen}

spielsweise: Ist das Ergebnis gut, wenn die Entscheidung von kompetenten Organisationsmitgliedern getroffen wurde? Müssen die Folgen einer Entscheidung immer positiv sein oder genügt es, wenn sie angemessen sind bzw. zur Organisation passen. Ist es nötig, dass der/die Entscheider/-innen ein hohes Commitment gegenüber der Entscheidung aufweisen? Ist die Qualität der Entscheidung schlecht, wenn die Folgen der Entscheidung nicht oder anders eintreten als geplant? Oder ist die Zufriedenheit der Beteiligten das Maß der Dinge?

Die Bewertung der Entscheidungsqualität bedarf gewisser Überlegungen:

\section{Die Bewertung könnte in Relation zu einer Refe- renzentscheidung erfolgen}

Problem: Es ist schwer, für die Bewertung der Entscheidung eine gleiche oder ähnliche Referenzentscheidung zu finden. Entscheidungen müssten vergleichbar sein, um sie beurteilen zu können. Da aber die Umweltbedingungen nicht laborstatisch sind, lässt sich kein „Erfolg“ messen. Für die Erfolgsbestimmung von Entscheidungen sind deshalb andere Entscheidungen als Referenzen oft nicht nutzbar. Als Referenzen können auch individuelle, 


\subsubsection{Bewertung von Entscheidungen}

organisationale oder gesellschaftliche Normen angesehen werden, die zur Bewertung von Entscheidungen und zur Diagnose von Defekten als relevant betrachtet werden (vgl. Woike 2007, S. 120).

\section{Messgrößen quantifizieren}

Um Entscheidungen messbar zu machen, müssten diese quantifizierbar sein. Entweder mittels eines reliablen Instruments oder anhand objektiver Messgrößen, wie Umsatz, Schulnoten o.Ä. (messgrößenbezogene Zustandsbeschreibung). Das Problem dabei ist, dass nicht jedes Entscheidungskriterium quantifizierbar und mathematisch abbildbar ist.

\section{Outputbewertung versus Prozessbewertung}

Die Sorgfalt, die Art der Durchführung und die daran Beteiligten könnten einen Maßstab für die Qualitätsbewertung von Entscheidungen darstellen. Problematisch daran ist allerdings, dass Entscheidungen von Natur aus inkonsistent sind, da ihre Entscheidungsverläufe sich aufgrund ihrer Vielfalt schlecht vergleichen lassen. Ein guter Prozess stellt noch lange keine gute Entscheidung dar. Zudem bleibt auch bei der Prozessbewertung eine 


\subsubsection{Bewertung von Entscheidungen}

Diskrepanz zwischen Erfolg und (intra- und interpersoneller) Wahrnehmung. Ein objektiver Misserfolg kann aber durchaus einen subjektiven Entscheidungserfolg darstellen, wenn sich z.B. die Unternehmenskultur oder die Motivation positiv verändert haben. In die Qualitätsbewertung von Entscheidungen fließen oft nur die Bewertung des Outputs, der Konsequenzen bzw. der Leistung ein, die am Ende „ausgeworfen“ wird. Eine Prozessbewertung könnte eine andere Perspektive auf die Qualität von Entscheidungen bieten.

Die Überlegungen 1 bis 3 werfen mehr Probleme auf, als dass sie Lösungen präsentierten. Um dennoch einen praktikablen Weg der qualitativen Einordnung von Entscheidungen einzuschlagen, wird folgende Vorgehensweise gewählt.

Einen Einordnungsversuch soll dafür die Abgrenzung von Entscheidungsfehlern und Fehlentscheidungen darstellen. Diese Herangehensweise ermöglicht es, eine Aussage zur Qualität der Entscheidung zu tätigen und eine allgemeine Abgrenzung vornehmen, ohne dass der Spielraum im Weiteren eingeschränkt wird. Die Darstellung der Begriffe Entscheidungsfehler und Fehlentschei- 


\subsubsection{Bewertung von Entscheidungen}

dung trennen zwei unterschiedliche Perspektiven, die die Qualität von Entscheidungen annähernd beleuchten können, ohne eine Messbarkeit vorzugeben oder sie dogmatisch zu bewerten.

Was ist ein Entscheidungsfehler im Vergleich zu einer Fehlentscheidung? Welche Kriterien grenzen die beiden doch sehr nah beieinander liegenden Begriffe voneinander $a b$, die häufig in Wissenschaft und Alltag gleich verwandt werden?

Wenn von einer Fehlentscheidung die Rede ist, wird impliziert, dass ein Fehler gemacht wurde. Der Begriff sagt etwas über die schlechte Qualität der Entscheidung und ihre Folgen aus. Die Folgen von Fehlentscheidungen werden oft als negativ bewertet, denn wenn die Auswirkungen als neutral oder schlecht bewertet würden, hätte es keinen "Fehler" bei der Entscheidung gegeben. Grundsätzlich liegt das Entscheidungsverhalten im Machtbereich der Organisationsteilnehmenden, d.h., die Bewertung des Entscheidungsrahmens und des Entscheidungsobjektes ist die Aufgabe der Organisationsteilnehmenden. Fehlentscheidung oder fehlerhaftes Entscheidungsverhalten unterliegt der Abweichung von der 


\subsubsection{Bewertung von Entscheidungen}

objektiven Entscheidungsrationalität. Eine Fehlentscheidung ist deshalb an Bedingungen geknüpft, die eine rationale Entscheidung erfüllen muss, und auf Fehler im Entscheidungsprozess zurückzuführen (vgl. Riesenhuber 2006, S. 38). Damit besteht ein Zusammenhang zwischen der Bewertung einer Entscheidung als Fehlentscheidung und deren nicht separat $\mathrm{zu}$ betrachtenden Auswirkungen. Dass Fehlentscheidungen Auswirkungen haben, liegt also auf der Hand. Doch Entscheidungen sind komplexe Phänomene, deren plakative Einordnung in gut und schlecht zuweilen schwerfällt. Schulz-Hardt (1997) grenzt die beiden Begriffe zwar nicht ab, gibt aber eine schlüssige Definition des Begriffs der Fehlentscheidung: Diese liege vor, wenn „der durch die Entscheidung und ihre Umsetzung erreichte Zustand substantiell den erwünschten Zustand verfehlt und diese Divergenz maßgeblich auf eine vorher getroffene Entscheidung zurückzuführen ist" (Schulz-Hardt 1997, S. 17). Diese Sichtweise zeigt deutlich den gescheiterten Zustand als Folge einer Entscheidung und legt den Fokus eher auf den Schlussakkord einer Teilaktivität des Entscheidungsprozesses, nämlich die Reflexion der Entscheidung (siehe Kapitel 6.3.6). Erst mittels der Reflexion wird sie zur 


\subsubsection{Bewertung von Entscheidungen}

Fehlentscheidung deklariert. Kahneman und Tversky (1979) lokalisieren die Voraussetzung für eine Fehlentscheidung in den ersten Phasen des Entscheidungsprozesses, der Problemwahrnehmung und der kollektiven Problemdefinition. Dort wird das Entscheidungsproblem aufbereitet und für die Auswahlentscheidung definiert, sodass die Alternativen ggf. vereinfacht oder gar reduziert werden. Sie geben damit auch zu erkennen, dass es sich um einen Entscheidungsfehler handeln könnte, und verankern diesen auch im Entscheidungsprozess.

Bei dieser ersten Betrachtung zeigt sich bereits die Schwierigkeit der klaren Einordnung und Abgrenzung in ein wissenschaftliches Definitionsraster. Die Güte einer Entscheidung kann aus verschiedenen Perspektiven unterschiedlich beurteilt werden (vgl. Haeften 1999, S. 44). In diese Beurteilung fließt oft das gesamte „Weltbild“ der Entscheider/-innen ein: Werthaltungen, Einstellungen, Erfahrungen und Präferenzen. Je nach Situation mag eine Entscheidung positiv, eine andere als Fehlentscheidung deklariert werden. Diese Subjektivität erschwert die Bewertung einer Fehlentscheidung. Ob und wann der gewünschte Zustand erreicht wird, entscheidet sich anhand der Zielsetzungen der Organisation bzw. des/der 


\subsubsection{Bewertung von Entscheidungen}

Entscheiders/-in. Je nachdem, welche Maßstäbe bzw. Normen angesetzt werden, wird die Entscheidung ggf. als eine Fehlentscheidung deklariert.

Im Gegensatz zur Fehlentscheidung steht bei einem Entscheidungsfehler der Prozess der Entscheidung im Fokus. Ein Entscheidungsfehler kann durchaus den Prozess der Entscheidung angreifen, aber das Entscheidungsergebnis kann sowohl objektiv als auch subjektiv zufriedenstellen. Im Experiment von Ahrens (1976, S. 27) sind alle Gruppenmitglieder von der getroffenen Entscheidung überzeugt, und der Zusammenhalt im Entscheidungsprozess hat das Gruppenklima verbessert, aber das Entscheidungsergebnis wurde als Fehlentscheidung deklariert. Wird der Prozess als nicht zufriedenstellend bewertet, kann es sich um einen Entscheidungsfehler handeln, der aber zu einer Fehlentscheidung führen muss. Die Fehlentscheidung kann durch objektive (messbare) Kriterien bewertet werden, und das Ergebnis fällt negativ aus, wohingegen bei Entscheidungen mit Entscheidungsfehlern auch subjektive Kriterien der Entscheidergruppe angesetzt werden müssen (vgl. Ahrens 1976, S. 27). Die subjektiven Kriterien ergeben sich aus dem Entscheidungsprozess (z.B. Zufriedenheit, Motivati- 


\subsubsection{Bewertung von Entscheidungen}

on). In einem Prozess mit Entscheidungsfehlern können subjektive und objektive Optimalität des Ergebnisses voneinander abweichen. Entscheidungsfehler zielen damit auf die Fehler im Prozess und nicht - wie bei der Fehlentscheidung - auf deren Ergebnis oder Ende.

Entscheidungsfehler beantworten die Frage danach, wie ein Entscheidungsprozess abgelaufen ist, d.h., ob es Defizite gab. Prozesse können Entscheidungsfehler aufweisen, sie führen aber nicht zwangsläufig zu einer Fehlentscheidung. Entscheidungsfehlern im Entscheidungsablauf haben lediglich eine erhöhte Wahrscheinlichkeit, dass die Entscheidung weniger objektiv ist, aber nicht zwangsläufig sein muss. Wo Fehlentscheidungen subjektiv im Auge des Betrachters sind, lassen sich Entscheidungsfehler besser objektivieren und operationalisieren. Deshalb ist die Einstufung als Fehlentscheidung eine reine subjektive.

Hierzu zwei Beispiele:

\section{Fehlentscheidung:}

Ein Unternehmen verlagert einen Unternehmensteil ins Ausland. Die Belegschaft wertet diese Entscheidung als Fehlentscheidung, weil dadurch Arbeitsplätze verlagert 


\subsubsection{Bewertung von Entscheidungen}

werden. Die Unternehmensleitung feiert die Entscheidung als Erfolg, weil das Stammwerk gerettet werden konnte und eine Kostenersparnis erzielt wurde.

\section{Entscheidungsfehler:}

Bei der Auslagerung eines Unternehmensteils wurde nicht der Kündigungsschutz des neuen Auslandswerkes berücksichtigt. Dadurch hatten alle neuen Mitarbeiter/innen am verlagerten Arbeitsstandort ein unbefristetes Arbeitsverhältnis und der Unternehmensleitung fehlte die Beschäftigungsflexibilität, mit der sie kalkuliert hatte.

Es bleibt festzuhalten, dass nicht jeder Entscheidungsfehler zu einer Fehlentscheidung führt. Zusammenfassend ergeben sich folgende Kriterien zur Abgrenzung einer Fehlentscheidung von einem Entscheidungsfehler:

- Fehlentscheidungen beziehen sich auf die Konsequenzen der Entscheidung im Prozess. Entscheidungsfehler hingegen beziehen sich auf Defizite im Entscheidungsprozess und können in jeder Phase der Entscheidung auftreten.

- Eine Entscheidung, die mit Entscheidungsfehlern im Prozess getroffen wurde, kann durchaus von 
zufriedenstellender Qualität sein ${ }^{16}$ auch wenn die Entscheidung als Fehlentscheidung gewertet wurden.

Nicht nur Fehlentscheidungen zeichnen sich als Produkte beschränkter Rationalität aus, sondern auch Entscheidungsfehler entpuppen sich als Ableitung der normativen Entscheidungstheorie, weil auch ihr Verhalten weiterhin intendiert rational ist. Die Individuen haben einen rationalen Anspruch an ihre Entscheidungsfindung. Diesen Anspruch haben auch Gruppen, wenn sie Entscheidungen treffen.

Als zentrale Fragestellung für die folgenden Kapitel sollen deshalb die Grundlagen der individuellen auf die kollektive Problemlösung übertragen und so die Besonderheiten von Entscheidungsfehlern auf Gruppenebene verdeutlicht werden.

${ }^{16}$ Beispiel: In der Feldstudie von Ahrens (1976, S. 27 f.) waren die Teilnehmenden einer Konferenz subjektiv mit ihrer Teilnahme zufrieden, obwohl die Erwartungen nicht erfüllt wurden. Das heißt, die Teilnehmenden waren zufrieden, obwohl sie sich im Vorfeld etwas anderes versprochen hatten. 


\subsection{Entscheidungen auf Gruppenebene}

Die organisatorischen Strukturen haben sich in den letzten Jahren verändert, so gibt es etwa komplexere Fragestellungen im Organisationsalltag, flache Hierarchien, Verschlankungen der Belegschaft etc., wobei die Organisationspolitik vermehrt auf Gruppenentscheidungen setzt. Dabei werden häufig die Vorteile der Zusammenarbeit in Gruppen gepriesen (vgl. Reimer et al. 2007, S. 7):

- Förderung von organisationalem und individuellem Lernen

- Gruppenmitglieder verfügen über mehr Wissensressourcen durch eine bessere Vernetzung.

- Motivationsverbesserung der Gruppenmitglieder

- Gruppenarbeit fördert den Zusammenhalt der Gruppe.

In vielen Organisationen erwächst die kollektive Entscheidungsfindung aus der Meinung, dass Gruppenentscheidungen qualitativ besser seien als Einzelentscheidungen. Es wird angenommen, dass die Gruppe dem 
3.2 Entscheidungen auf Gruppenebene

qualifiziertesten Gruppenmitglied überlegen sei (vgl. Rosenstiel 1992, S. 329). Gruppenentscheidungen sollen, isoliert auf eine Einzelentscheidung heruntergebrochen, qualifizierter sein als die jeweilige Einzelentscheidung, u.a., weil Einzelentscheidungen bei einer zu treffenden Gruppenentscheidung einem „größeren Ganzen“ gegenüberstehen, das es dem Einzelnen „verbietet“, ichbezogene Ansätze zu verfolgen (vgl. Schüßler 1993, S. 2 f.). Entscheidungen von Gruppen haben eine demokratischkollektive Natur und könnten deshalb bei den Organisationsmitgliedern eher Akzeptanz finden. Neben den beschriebenen positiven Faktoren (siehe Kapitel 3.1.1.) gibt es eine Reihe weiterer positiver Befunde: steigende Motivation und Arbeitszufriedenheit, durch positive Synergie bedingte Produktivitätserhöhung, Erweiterung von Fähigkeiten und Fertigkeiten, verbesserte Kommunikation, mehr Bindung an die Organisationsziele und stärkerer Fokus auf Prozesse statt Funktionen (vgl. Weinert 2004, S. 449). Die Inhaltsanalyse von Davis (1992, S. 3 f.) zeigt jedoch auch das Gegenteil: Soziale Interaktion führt nicht generell zu besseren Entscheidungen. Andere Forschungen ergaben, dass z.B. Brainstorming von Einzelpersonen effektiver ist als Brainstorming in Gruppen (vgl. 
3.2 Entscheidungen auf Gruppenebene

Reimer et al. 2007, S. 7). Die verbreitete Ansicht, dass nur der vollständige Informationsaustausch in Gruppen zu qualitativ guten Ergebnissen führe, konnte (u.a. von Reimer et al. 2007) widerlegt werden. Zum einen, weil Gruppen die vorhandene Menge an Informationen nicht systematisch nutzen, und zum anderen, weil die Auswahl der Informationen durch die Gruppe sozial geprägt ist. Die Gruppenentscheidungen und ihre Einflüsse erscheinen demnach genauso undurchsichtig wie Einzelentscheidungen.

Trotz der Vorteile, die die Gruppenarbeit in sich birgt, sind Zeitdruck, unvollständige Informationen oder Ungewissheit ihre Fallstricke. Es werden Entscheidungen in Gruppen getroffen, ohne dass die Risiken vorher abgewogen wurden. Auch bei Gruppenentscheidungen können Defekte die Entscheidungsfindung mehrerer Teilnehmenden beeinträchtigen (vgl. Eisenführ/Weber 2010, S. 312). Vor diesem Hintergrund werden Entscheidungsregeln auch für Gruppen gefordert, die ein Handeln in komplexen Situationen und von Menschen ohne technische Unterstützung ermöglichen sowie strukturelle Unsi- 
3.2 Entscheidungen auf Gruppenebene

cherheiten und Zeitdruck als Grenzen überwinden (vgl. Woike 2007, S. 132).

Entscheidungsprozesse sind oft verzweigt, undurchsichtig und wenig greifbar. Vielfältige Effekte machen einer rationalen Entscheidung einen Strich durch die Rechnung, denn die Rationalität menschlichen Handelns ist durch die menschliche Psyche begrenzt (vgl. Riesenhuber 2006, S. 69). Ursächlich für nicht rationales menschliches Verhalten, gewinnen Entscheidungsfehler mit dem Wissen um sie an Bedeutung und führen zu einer Bewusstwerdung des nicht objektiven Handelns von Organisationsteilnehmenden und Entscheidungsträgern/innen. Nicht rationales Verhalten stellt nach Meinung einiger Autoren/-innen eine grundsätzliche Gefährdung des Entscheidungsprozesses dar (u.a. Janis 1989, S. 159 f.). Es wird damit eine negative Qualität der Entscheidung vorgegeben, wenn Entscheidungen durch Handlungsdefizite eingeschränkt werden.

Gruppen wie auch Einzelpersonen treffen Entscheidungen, die nicht ihrem Leistungsniveau, Qualifikationen und Kenntnisstand entsprechen. Beispiele: Organisationsmitglieder entscheiden sich für Bewerber/-innen, ohne deren Vita und Persönlichkeit aus einem einstündigen Vorstel- 
3.2 Entscheidungen auf Gruppenebene

lungsgespräch ausreichend zu kennen; Schulungen werden geplant, ohne die genauen Inhalte zu kennen; Manager entscheiden aus dem Bauch heraus über Millionen innerhalb von wenigen Minuten etc.

Entscheidungen werden tagtäglich getroffen, trotz des Risikos, nicht alle Informationen zu kennen. Wie kommt es, dass Organisationen trotzdem handlungsfähig bleiben? Grundsätzlich müsste es im Entscheidungsprozess viele Gefahrenquellen und Fallstricke geben, die die Rationalität und Qualität von Entscheidungen beeinflussen, sodass gute Entscheidungen eher dem Zufall entsprechen? Eine rationale Entscheidungsfindung erscheint weit weg vom Alltag ${ }^{17}$ und bedarf deshalb einer Untersuchung, die ein Entscheidungshandeln in Gruppen nicht verteufeln oder zu positiv hervorheben soll. Es stellt sich die Frage, ob heuristische Entscheidungen eine Antwort

17 Entscheidungsfehler, Hindernisse, Prozessverluste, Defekte: Diese Begriffe stehen grundsätzlich nicht für das Gleiche. Ihnen ist aber gemein, dass die Konnotation eine Messlatte für die Qualität des Agierens ist bzw. das Defizitäre im Handeln betont. Es bleibt aber festzuhalten, dass Defekte als negativ für die Rationalität der Entscheidung bewertet werden und Entscheidungen veranlassen, deren Konsequenzen die handelnden Personen oft selbst nicht wünschen (vgl. Martin 2012, S. 12). 
auf die vielen Defekte im Entscheidungshandeln der Gruppe sind oder im Gegenteil einen Fallstrick darstellen.

\subsection{Heuristiken im Allgemeinen}

Entscheider/-innen treffen häufig Annahmen, ohne sich ein umfassendes Bild gemacht zu haben. Brauchbar wären klare Entscheidungshilfen wie mathematische Wahrscheinlichkeitsberechnungen, Expertengutachten o.Ä., die stets zur Hand sind. Dies ist jedoch selten der Fall. Entscheidungen sind allein schon durch die kognitive Begrenzung menschlicher Informationsverarbeitung eingeschränkt. Entscheidungen werden deshalb häufig intuitiv und abgekürzt getroffen, weil ihre begrenzte Informationsverarbeitung und -aufnahme sowie Zugangsschwierigkeiten zu notwendigem Wissen die Entscheidungsfindung zu einer heuristischen Entscheidungsfindung transformieren. Deshalb bleibt den Organisationsteilnehmenden häufig keine Wahl; sie müssen sich heuristisch entscheiden. 
Ein Beispiel für Heuristiken ${ }^{18}$ aus dem Bereich der Medizin geben Gigerenzer und Todd (1999a, S. 30): Ein Patient kommt mit Brustbeschwerden ins Krankenhaus. Der Arzt in der Notaufnahme muss anhand weniger Informationen über die weitere Behandlung entscheiden. Eine Entscheidung über Leben und Tod aufgrund lediglich einer Handvoll Informationen zu treffen, erscheint zunächst unverantwortlich. Wegen Zeitmangels und fehlender Informationen bleibt jedoch oft keine andere Wahl. Der Arzt entscheidet innerhalb weniger Minuten über lebensrettende Maßnahmen, die das Risiko einer Fehlentscheidung bergen können. Dies gilt sowohl in ernsten Notfällen als auch bei Alltagsentscheidungen. Kein Individuum kann sich von dem Phänomen der Heuristik frei machen, das täglich mehrfach Anwendung findet. Es wird eine Vielzahl von Heuristiken ständig verwendet.

Welche Rolle Heuristiken bei der Urteilsbildung spielen, soll anhand dreier Beispielheuristiken verdeutlicht werden:

${ }^{18}$ Heuristik kommt aus dem Griechischen und bedeutet „finden“. 
- Wenn ein Ereignis für eine Population von hoher Bedeutung ist, erhöht sich die subjektive Wahrscheinlichkeitseinschätzung. Beispiel: Wenn Personen schätzen sollen, welchen Beruf jemand ausübt, nimmt die zu schätzende Wahrscheinlichkeit, mit der ein Mensch einen bestimmten Beruf ausübt, zu, wenn die Vorstellung des Schätzenden über die Berufsgruppe (wie Stereotype bzgl. Buchhaltern/-innen) mit den tatsächlichen Merkmalen dieser Gruppe übereinstimmt (vgl. Lindstädt 1997, S. 61).

- Je eher Menschen Beispiele für das Auftreten von Situationen finden, desto höher schätzen sie die Wahrscheinlichkeit des Ereignisses ein, d.h., sie über- oder unterschätzen das Auftreten, weil sie die Situation bzw. das Risiko, z.B. durch Berichte über Falschfahrer auf der Autobahn oder über Flugzeugabstürze (vgl. Tversky/Kahneman 1979), kennen.

- Eine Information wird als Anker verwendet und so angeglichen, dass sie zur Vorhersage passt. Der Anfangswert wird als Maßstab für Urteile benutzt und dabei schrittweise angepasst. 
3.3 Heuristiken im Allgemeinen

Tversky/Kahneman (1974) ermitteln durch ein Glücksrad einen Anfangswert. Der zu schätzende Wert bleibt meist in der Nähe des mit dem Glücksrad zufällig ermittelten Anfangswertes.

Die Beispiele sind in der Reihenfolge Repräsentationsheuristik, Verfügbarkeitsheuristik und Anker-Heuristik aufgelistet und veranschaulichen die Natur von Heuristiken. Grundsätzlich wird aber oft davon ausgegangen, dass, je breiter die Informationsbasis ist, desto besser sind die Entscheidungen. Denn Informationssuche und -bewertung sind im allgemeinen Handeln zentrale Grundlagen für jedwedes Entscheidungsverhalten. Denn wenn der/die Entscheidungsträger/-in im Entscheidungsprozess mit einem zu lösenden Problem konfrontiert wird, entwickelt er/sie zunächst ein Informationsbedürfnis (vgl. Riesenhuber 2006, S. 62). Zum Zeitpunkt der Entscheidung fehlt aber oft relevantes Wissen, um die Situation ausreichend einschätzen zu können. Je mehr Informationen der/die Entscheider/-in hat, desto weniger Unsicherheit fühlt er/sie im Entscheidungsprozess. Heuristiken beruhen aber auf erstaunlich wenigen Informationen und erscheinen trotzdem als vertrauenswürdig (vgl. Neumer 
2009, S. 30). Welche Informationen vorhanden sind, ergibt sich aus der Zielsetzung der Gruppe sowie den Fähigkeiten und dem Wissen ihrer Teilnehmenden. Daraus kann sich eine unterschiedliche Wissensbreite und qualität sowie Tiefe der Information zu den einzelnen Entscheidungsoptionen ergeben.

Gründe, warum Entscheidungswege abgekürzt werden, gibt es viele. Aber heuristische Entscheidungen entstehen, weil die Organisationsmitglieder ihre Informationssuche beschneiden, wenn sie z.B. meinen, bereits genügend Wissen zu haben, um eine Entscheidung treffen $\mathrm{zu}$ können, oder weil sie denken, dass sie eine ähnliche Situation bereits erlebt haben, und auf ihr Erfahrungswissen zurückgreifen wollen oder weil innen die Komplexität des Prozesses nicht deutlich ist. Heuristische Entscheidungen werden vor allem getroffen, wenn

- nicht alle Informationen jederzeit verfügbar oder zu viele abrufbar sind,

- nicht alle Informationen kognitiv abrufbar sind,

- ein hoher Handlungsdruck, z.B. aus Zeitmangel oder Unsicherheit, besteht, 
- keine umfassenden Informationen benötigt werden (z.B. beim Erfahrungshandeln von Experten/-innen).

Heuristischen Entscheidungen führen zu

- einer begrenzten Aufnahme der Informationsmenge,

- einer limitierten Wahrnehmung,

- einer Selektion von Informationen unter Berücksichtigung individueller Präferenzen, Einstellungen, Erfahrungen und Werthaltungen.

Die heuristische Entscheidungsforschung räumt mit der Behauptung der vollständigen Informationssuche und bearbeitung auf und meint, dass die Einfachheit der eingeschränkten Informationssuche der Anpassung an die Umwelt diene (vgl. Gigerenzer 2007, S. 26). Heuristiken sind gerade von Vorteil, wenn nur wenige Informationen vorliegen. Heuristiken sind Unsicherheitsurteile und keine exakt geprüften Wahrscheinlichkeiten von Konsequenzen und deren Abwägungen (vgl. Jungermann 2009, S. 45). Heuristiken sind robuste Konstrukte, die dazu führen, dass Menschen nicht überlastet werden und trotz kom- 
3.3 Heuristiken im Allgemeinen

plexer Umweltbedingungen eine optimale Lösung finden können, ohne Wahrscheinlichkeitsrechnungen oder Computerauswertungen bzw. statistische Analysen zu nutzen (vgl. Gigerenzer/Gaissmaier 2006, S. 25). Sie sind schnell, sparsam und transparent (fast and frugal). ${ }^{19}$ Die Kriterien für eine heuristische Auswahl hin zu einer Lösung bemessen sich nach einem sehr individuellen (kognitiven oder emotionalen) Katalog, den das Individuum aus seinen Präferenzen, Einstellungen, Werthaltungen oder Erfahrungen herleitet. Diese können z.B. Geschmäcker bei Kaufentscheidungen oder das Wiedererkennen der Unternehmensmarke bei komplexen Börsengeschäften sein. Andersherum können bestimmte Informationen auch eine limitierte Auswahl der Lösungsmöglichkeiten hervorrufen. Bereits der Prozess der Informationsverarbeitung ist heuristisch geprägt, nicht nur die Entscheidung selbst. Denn auch die Informationssuche und -

19 Auch wenn die Argumente, auf denen die heuristische Entscheidung fußt, transparent erscheinen, sind die Mechanismen, die dahinter wirken, alles andere als eindeutig identifizierbar. Entscheider/-innen können oft nicht einmal sagen, warum sie diese oder jene Entscheidung getroffen haben, auch wenn sie die Argumente dafür nennen können. Gigerenzer (2007, S. 145 f.) thematisiert dies unter der Überschrift „Ein einziger guter Grund reicht". 
bewertung ist eng verzahnt mit den Präferenzen des Individuums. Heuristische Entscheidungsprozesse sind stark geprägt durch dieses Präferenzsystem, das anhand weniger (aber wichtiger) Informationen in der Lage ist, eine Entscheidung angemessen zu treffen. Der Cut-offEffekt ist hierbei besonders hervorzuheben, da er Prioritäten setzt und für den/die Entscheider/-in eine Entscheidung forciert, die nicht die Informationstiefe hat, die aber trotzdem im Sinne von Machbarkeit und Zeit sowie vor dem Hintergrund der eigenen Präferenzen angemes$\operatorname{sen}^{20}$ getroffen wird. Bei dem Cut-off-Effekt ist der Handelnde in der Lage, in kurzer Zeit effektive, wenn auch nicht optimierte Entscheidungen zu treffen, weil er keinen

${ }^{20}$ Auch March (1994) geht eher davon aus, dass Entscheider/-innen in Organisationen Entscheidungen eher nach ihrer Angemessenheit treffen. Der Fokus liegt bei dieser organisationspsychologischen Sichtweise auf der personellen und organisationalen Organisationsidentität (z.B. Rollenerwartungen), statt auf der reinen der Bewertung der Konsequenzen einer Entscheidung. March (1994, S. 60): „Organizational rules define what it means to be an appropriate decision maker." "They are thaught appropriate codings of situations and appropriate responses to them." (S. 62). Das heißt, nach der „Logik der Angemessenheit" stellt sich eine Person bei der Konfrontation mit einem Problem folgende Fragen: Was ist organisationsseitig angemessen? Welche Art von Situation ist dies? Und was tut eine Person wie ich in einer solchen Situation? (Regeln) (vgl. Moser et al. 2000, S. 441). 
Vergleich der Alternativen vornimmt, sondern die Informationssuche abbricht, indem er eine Alternative wählt, die alle Kriterien erfüllt (vgl. Koppers 2013, S. 37). Von Haeften (1999, S. 61 ff.) hat den Cut-off-Effekt in einer Studie am Beispiel von Wohnungssuchenden nachgewiesen: Wenn der zu höhe Mietpreis für eine Wohnung die anderen attraktiven Wohnungsattribute überlagert, führt dieses Kriterium zu einer Eliminierung der Entscheidungsalternative einer bestimmten Wohnung als Entscheidungswahl.

Neben den Informationen und der individuellen „Entscheiderwelt" wirkt auch die Umwelt auf die Lösungsmöglichkeiten. Die Gestaltung des Umfeldes ist z.T. sehr situativ (z.B. Temperaturgegebenheiten, Zusammensetzung der Gruppe, Zeitpunkt der Entscheidung etc.). Heuristiken fügen sich in die natürliche Umgebung von Individuen (vgl. Woike 2007, S. 132) und machen diese oft erst handlungsfähig.

Informationsüberfluss, Zeitdruck usw. Iassen Personen heuristisch handeln, indem das verfügbare Wissen nutzbar gemacht wird, um eine organisationsangemessene Entscheidung zu generieren. Angemessenes organisati- 
3.3 Heuristiken im Allgemeinen

onales Entscheiden meint, dass eine Situation zu einer erfolgreichen Umsetzung der Organisationsziele beiträgt und/oder zu zufriedenstellenden Ergebnissen für die Organisationsteilnehmenden führt.

Beispiel: Wenn eine Umweltschutzorganisation ein Einsatzteam für ein Projekt plant, um etwa einen Trawler von Walfängern zu stoppen, ist das Organisationsziel erfüllt, wenn das Walfangschiff an seinem Tun gehindert und die Öffentlichkeit durch rege Medienarbeit informiert wurde. Heuristiken kommen in dieser Situation zum Einsatz, indem das Projektteam in einer vorgegeben Zeit (Trawler läuft am Tag $x$ aus) zahlreiche kleine Entscheidungen trifft, ohne dass alle Informationen in die Bewertung des Projekts einfließen können (wie sehen die landestypischen Gesetze aus? Welche Gefahren gehen von den Behörden aus? Welche Rechte haben wir bei Inhaftierung der Aktivisten/-innen?). Angemessen ist die Entscheidung dann, wenn die Projektteilnehmenden mit ihrer Zielerreichung zufrieden sind. Der Grad dieser Zufriedenheit ist an die Umstände der Entscheidungssituation gekoppelt. Wenn unter den gegebenen Umständen „nicht mehr zu machen war" (starker Wellengang, Gegenwehr etc.) kann die Zielerreichung auch von der vorherigen 
3.3.1 Qualität heuristischer Entscheidungen

Planung abweichen, und die Teilnehmenden verbuchen das Projekt trotzdem als Erfolg.

Abgeleitet von der Frage, wie die Qualität heuristischer Entscheidungen ist, soll im nächsten Kapitel die Frage diskutiert werden, ob Heuristiken Entscheidungshilfe oder Entscheidungsdefekte sind.

\subsubsection{Qualität heuristischer Entscheidungen}

Wann heuristische Entscheidungen getroffen werden und wozu sie führen, würde lediglich eine allgemeingültige Aussage ergeben. Jedoch stellt sich die weitaus interessantere Frage, ob Heuristiken zu den Entscheidungsdefekten zählen oder ob es sich um eine hilfreiche und „allgegenwärtige" Bauchentscheidung handelt, die in Gestalt einer Entscheidungshilfe daherkommt? Es stellt sich also die Frage, ob Heuristiken Entscheidungsfehler oder Entscheidungshilfen sind. Die Antwort soll der Ableitung dienen, heuristische Entscheidungen als wertvoll (Entscheidungshilfe) oder als hinderlich (Entscheidungsdefekt) für das spätere Kapitel Personalauswahl (als Wirkungsfeld) zu sehen. 
3.3.1 Qualität heuristischer Entscheidungen

Simon wurde 1978 der Nobelpreis für Wirtschaftswissenschaften u.a. für die Begründung verliehen, dass optimale Entscheidungen einen Ausnahmefall im menschlichen Entscheidungsverhalten darstellen (vgl. March/Simon 1958, S. 140 f.). Der/die Entscheider/-in sucht unter den inm/ihr zugänglichen Informationen solange die Alternativen, bis er/sie auf eine hinreichende (anstelle der optimalen) Entscheidungsalternative stößt und sich dann für diese entscheidet (vgl. Hinrichs 2011, S. 77). Handelnde gehen selten den langen, steinigen Weg der vollständigen Informationssuche und -verarbeitung, sondern verkürzen ihr Bemühen. Grundsätzlich könnte der Ansicht gefolgt werden, dass eine Entscheidung mangelhaft sei, wenn nicht die optimale und nutzenmaximierende Entscheidung getroffen wurde. Der Paradigmenwechsel, statt rationaler auch emotionale Aspekte bei Entscheidungen zuzulassen, basiert u.a. auf den Arbeiten von Gigerenzer, Todd und der ABC-Gruppe (1999). Heuristiken galten lange als Entscheidungsdefekte, weil die Gefahr einer nicht rationalen Entscheidung auch eine Fehlentscheidung begünstigen kann und zu systematischen Verzerrungen oder Fehleinschätzungen führen kann (vgl. Hinrichs 2011, S. 84). Heuristisches Entscheiden gemäß 
3.3.1 Qualität heuristischer Entscheidungen

dem Heuristics-and-bias-Ansatz basierte auf Urteilsfehlern (vgl. Tversky/Kahneman 1974, Kahneman/Klein 2009). Aber ist dem auch wirklich so? Zunächst einleitend zur Qualität der heuristischen Entscheidung.

Es gibt eine große Anzahl von Heuristiken, die als mögliche Denkfehler, Täuschungen, Fehlleistungen und Missgeschicke betrachtet werden (vgl. Scholz et al. 2003, S. 21 f.) und weniger als Entscheidungshilfe. Um diese Frage zu klären, wird eine qualitative Bewertung von Gruppenentscheidungen benötigt. Rosenstiel und Nerdinger (vgl. 2011, S. 346) machen die Entscheidungsqualität z.B. abhängig von der Komplexität eines zu bearbeitenden Problems, dem zu erwartenden Zeitaufwand oder der Zusammensetzung der Gruppenmitglieder. Oder kommt es auf die Beurteilung der Situation im Ganzen an bzw. darauf, vor welchem Hintergrund, in welcher Zeit und mit welchen Teilnehmenden die Entscheidung abläuft?

Wie bereits im vorherigen Kapitel erwähnt, ist eine weitverbreitete Ansicht, dass, je mehr Informationen wir bei der Entscheidungsfindung berücksichtigen, unsere Entscheidungen desto besser ausfallen (vgl. Reimer et. al. 2007, S. 8). Ein Zuviel an Informationen (information 
3.3.1 Qualität heuristischer Entscheidungen

overload) soll die Informationsverarbeitung und Problemlösung gar beeinträchtigen. Heuristiken beinhalten deshalb den Mut zum Risiko bei der Abkürzung langer und beschwerlicher Lösungswege, das Eingehen auf die Besonderheiten einer bestimmten Problemsituation, die Nutzung eigener Erfahrungen sowie der Empfehlungen anderer (Groner/Groner 1991, S. 318, zit. n. Woike 2007, S. 12). Heuristiken als naive Entscheidungswege zur Entscheidungsfindung zu sehen, wird von einigen Vertretern/-innen des Simple-heuristics-Ansatzes als nicht mehr angemessen oder gar als falsch herausgestellt. Ihrer Ansicht nach kann eine Entscheidungssituation auch ohne vollständige Informationsbasis ganzheitlich betrachtet werden. Heuristiken vereinfachen lediglich die Bedingungen, die das Entscheidungsproblem sowie seine Konsequenzen kognitiv und emotional überlasten, und entledigen sich des „Ballasts“. Sie sehen Heuristiken durchaus als effiziente Verarbeitungsmechanismen, insbesondere hinsichtlich des zeitlichen Aufwandes (u.a. Hinrichs 2011, S. 83), jedoch ohne sie als Heilsbringer zu feiern.

In einer Befragung von Gigerenzer (2007) wurde 100 Personen, die ein durchschnittliches Wissen über Aktien 
3.3.1 Qualität heuristischer Entscheidungen

besaßen, die Frage gestellt, welche 50 Aktiennamen sie kennen. Aus ihren Nennungen wurde ein Portfolio entwickelt. Das Portfolio nahm an einem Wettbewerb (ähnlich einem Planspiel) teil und erzielte dabei bessere Gewinne als 88\% der anderen 10.000 eingesandten Portfolios. Diese und ähnliche Forschungsergebnisse über die Qualität von Heuristiken sollten das einstmalige Verteufeln von abgekürzten Entscheidungswegen widerlegen. Denn auch wenn es sich um Vereinfachungen handelt, können deren Ergebnisse unter gewissen Umständen durchaus als korrekt angesehen werden (vgl. Gigerenzer 2007, S. 52). Dennoch bergen sie Risiken, die die Qualität der Entscheidung negativ beeinflussen können, z.B. durch übertriebenes Selbstbewusstsein in Bezug auf die eigenen Entscheidungen, Fehlinterpretationen oder Entscheidungsdefekte.

Es lassen sich zahlreiche Argumente für beide Ansichten (Defekt versus Hilfe) finden. Im Folgenden soll dieser Frage mit verschiedenen Argumenten nachgegangen werden. Drei Aspekte differenzieren den Entscheidungsdefekt von der Entscheidungshilfe:

1. Angemessenheit 
3.3.1 Qualität heuristischer Entscheidungen

2. Kausalität des Handlungserfolges

3. Ökologische Rationalität

Heuristiken reduzieren die Menge an Informationen und Reizen. Deshalb werden sie als kognitive Daumenregeln angewandt, wenn Organisationsteilnehmende unter Unsicherheit hinsichtlich der künftigen Konsequenzen Entscheidungen treffen müssen. Heuristiken haben dabei die Aufgabe, diese Informationen möglichst effizient $z u$ verarbeiten, aber ohne diese zu simplifizieren. Auch in Simons Konzept der Begrenzten Rationalität (1959) wird die vollkommene Transparenz fallengelassen und der/die Entscheidungsträger/-in hilft sich durch eine Vereinfachung des Entscheidungsproblems (vgl. Behrens 1980, S. 46). Eine Vereinfachung birgt immer das Risiko, wichtige Alternativen oder Informationen, die im Prozess der Entscheidung ausgeklammert werden, „unter den Tisch“ fallen zu lassen und damit vielleicht entscheidende Alternativen nicht wahrzunehmen. Genauso verhält es sich mit der Reduzierung des Informationsgehaltes. Auch sie birgt die Gefahr, entscheidende Informationen nicht in den Entscheidungsprozess einzubeziehen. Der heuristische Entscheidungsprozess ist aber maßgeblich durch 
3.3.1 Qualität heuristischer Entscheidungen

solche Abkürzungsprozesse gekennzeichnet. Indem Informationen und Reize bei der Problemlösung übersehen werden und eigene Präferenzen die Sicht des/der Entscheiders/-in trüben, schleichen sich Ungereimtheiten in die Entscheidung ein, die bei einer vollständigen Informationsumgebung nicht vorhanden wären. Einzelne Teilaktivitäten des Entscheidungsprozesses werden zusammengefasst, umgangen oder weggelassen. Dies bezeichnen Grünig und Kühn (2012, S. 65 f.) als „heuristische Schlaufe“. Deren Natur es ist, dass sie in allen Prozessschritten vorkommen können, d.h. in jeder Teilaktivität kann es zu heuristischen Schlaufen kommen (siehe Abbildung 3).

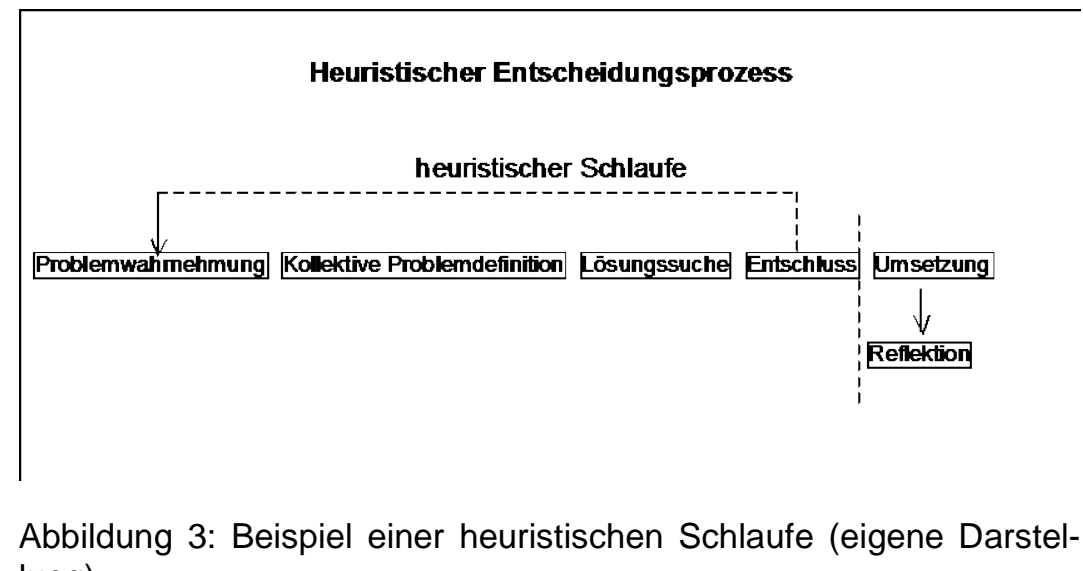
lung). 
3.3.1 Qualität heuristischer Entscheidungen

\section{Angemessenheit}

Der große Vorteil der heuristischen Entscheidung ist ihre hohe ökologische Validität, d.h. die Übertragbarkeit auf den Organisationsalltag. Dem steht gegenüber, dass die heuristische Entscheidungsforschung häufig unter Testbzw. Laborumgebungen betrieben wird. Hierbei kann es zu Verzerrungen kommen, und Heuristiken können als Defekte interpretiert werden, weil die Entscheider/-innen Informationen außer Acht gelassen haben. Dies liegt in der Natur der Laborforschung. Bei der Auffassung des Menschen als eines „collective rational being" wird angenommen, dass menschliche Fähigkeiten und Leistungen sich nur in einem gesellschaftlichen Rahmen verstehen und bewerten lassen, d.h., ob und inwieweit eine einzelne Entscheidung vernünftig ist, lässt sich somit nicht isoliert im Labor, sondern nur in praktischen Kollektiven betrachten (vgl. Scholz et al. 2003, S. 16). Es wird aber oft von Fehlentscheidungen ausgegangen, wenn ein bestimmtes Ergebnis nicht eintrifft oder ein gewisses Ziel nicht erreicht wird. Aufgabenumwelten mit ökologischer Validität sind aber oft komplexer Natur; in innen gibt es oft keinen Fixstern der Zielerreichung oder der Ergebniserzielung, sondern die Zufriedenheit fungiert als Maß- 
3.3.1 Qualität heuristischer Entscheidungen

stab. Meist zeigen sich auch erst nach Jahren die Wirkung und damit ihr Wert. Eine getroffene Entscheidung führt nicht automatisch zu einer Problemlösung (vgl. Neumer 2009, S. 13), aber kann trotzdem zur Zufriedenheit der Organisationsteilnehmenden sein.

Ein Mehr an Informationen muss nicht immer besser sein (vgl. Gigerenzer 2007, S. 29 ff.), wenn die komplexe und zeitraubende Optimierungstendenz von Entscheidungen einer beschränkt rationalen Entscheiderwelt weicht, in der Präferenzen, Emotionen, Zeitdruck, Lernprozesse etc. eine Rolle spielen können. Der Handlungserfolg von Heuristiken soll deshalb eher als effiziente Handlungsentlastung angesehen werden. Mit dem Anspruchsniveau der Organisationsteilnehmenden, d.h. den Maßstäben oder den Zielen ihrer „Entscheiderwelt“, geht auch die Bewertung der Entscheidung einher. Wenn die Organisationsteilnehmenden eine in ihrem Sinne angemessene und ausreichende Entscheidung treffen, kann nicht mehr von einem Entscheidungsdefekt in der Auswahl ausgegangen werden. Bei der heuristischen Entscheidung handelt es sich um ein angemessenes Anspruchsniveau, das als ausreichend angenommen werden kann (vgl. Behrens 1980, S. 46). 


\subsubsection{Qualität heuristischer Entscheidungen}

Deshalb soll die Angemessenheit als Entscheidungskriterium der Bewertung der Entscheidungsqualität dienen. Damit ist gemeint, dass die Mitglieder einer Gruppe bestrebt sind, mit ihren eigenen Entscheidungsregeln ein angemessenes Ergebnis zu finden (vgl. Reimer et. al. 2007, S. 27). Heuristiken sind damit Modelle beschränkter Rationalität (vgl. Reimer et al. 2007, S. 26). Als solche sind sie zunächst weder gut noch schlecht (vgl. Gigerenzer 2007, S. 58), weder rational oder irrational, da ihr Wert von dem Kontext abhängt, in dem die Heuristik verwendet wurde. Ein angemessenes und ausreichendes Ergebnis des Entscheidungsprozesses ermöglicht der Organisation eine andere Sichtweise auf ihr Handeln. Wenn sie ihre Entscheidungen bewerten, brauchen diese nicht das maximal mögliche Ergebnis zu sein, sondern sollte im Rahmen des Machbaren liegen, also angemessen sein. Jede nicht optimale Entscheidung wäre ansonsten (im Sinne einer rationalen Betrachtung) als defizitär einzustufen. Die Sichtweise eines angemessenen, zufriedenstellenden Anspruchsniveaus befreit vom Stigma der Perfektion bzw. der „echten“ Rationalität und garantiert eine gewisse Freiheit und einen Spielraum im Organisationshandeln. Statistische Sichtweisen helfen 
3.3.1 Qualität heuristischer Entscheidungen

modernen, aufgeschlossenen Organisationen nicht weiter, gerade in der vernetzten und digitalen Welt sind Grenzen des Geistes und standardisierte Entscheidungsverfahren wenig förderlich für kreative Organisationsprozesse mit Entscheidungen, die neue Marktanteile entstehen lassen.

\section{Kausalität}

Eine weitere Betrachtung, die beide Ansichten hinterfragen kann und bereits bei der Angemessenheit anklang, ist der Kausalitätsansatz. Es geht dabei um eine kausale Verlinkung von Entscheidung und Entscheidungsbewertung. Eine Bewertung kann erst erfolgen, wenn ein Handlungserfolg kausal auf die Entscheidung zurückzuführen ist. Wenn heuristische Entscheidung und Handlungserfolg unabhängig voneinander gesehen werden, stellt sich nicht die Frage nach dem Defekt. Defekte sind nicht nur ein prozessuales Element, sondern können auch eine Wertung des Handlungserfolges sein. Die Entscheidung ist erst defekt, wenn der Handlungserfolg gezeigt hat, dass es sich um eine defekte Entscheidung gehandelt hat. Heuristiken sind danach Entscheidungsregeln für alle Arten von Entscheidungen, die das Problem der 
3.3.1 Qualität heuristischer Entscheidungen

Komplexität für das Individuum lösen. Entscheidungen ohne Verlinkung mit dem Entscheidungserfolg können nicht als Defekte dargestellt werden. Da diese Darstellung in der (ökologischen) Realität schlecht gelingt, bleibt die Heuristik eine Entscheidungshilfe für ihre Anwendenden. Für sich allein steht die Entscheidung zunächst separat da. Erst durch die Verbindung von Kausalität und Entscheidungsbewertung (Handlungserfolg) kann die Heuristik (und jede andere Entscheidung) zum Defekt werden.

Um diesen Gedanken nochmals zu unterstreichen, sei als Beispiel die „Personalauswahl“ genannt, da die Folgen einer solchen Personalentscheidung sich oft erst in Jahrzehnten zeigen und viele Parameter den Erfolg der Entscheidung ins Gegenteil verkehren können. Der Bewerbungsprozess wurde vielleicht unter Zeitdruck entschieden, da der/die einstige Stelleninhabende sein/ihr Arbeitsverhältnis gekündigt hat, sodass die Gruppe sowohl einige Bewerber/-innen als auch einige Unterlagen nicht anhören bzw. sichten konnte. Ihre Entscheidung wurde deshalb unter Berücksichtigung der vorhandenen Informationen getroffen. Damit ist sie nicht als ursächlich 
3.3.1 Qualität heuristischer Entscheidungen

für einen künftigen Handlungserfolg anzusehen. Selbst wenn sich später herausstellt, dass der erwünschte Erfolg nicht eingetreten ist, gelingt der Beweis, dass dies ursächlich für eine fehlerhafte heuristische Entscheidung ist, schwerlich. Die Bewertung von Entscheidungserfolgen ist in der Realität schwierig und insbesondere bei Personalentscheidungen wenig greifbar. Ist der/die eingestellte Kandidat/-in in den ersten Wochen noch hoch motiviert, lässt sein Engagement langsam nach. Die Gründe dafür müssen nicht in der schlechten Auswahl des Entscheidungsgremiums liegen, sondern können auch mit einer schlechten Einarbeitung, Nichteinhalten des psychologischen Vertrages ${ }^{21}$ zwischen Arbeitgeber und Arbeitnehmer o.Ä. liegen. Denn gerade die ökologische Validität verhindert eine Darstellbarkeit des Entscheidungserfolges in Verbindung mit der Entscheidung. Im Gegensatz zum heuristischen Entscheidungsprozess sind bei der rationalen Entscheidung die Parameter durch die Mitglieder kontrollierbar und diesen bekannt, sodass eine kausale Verlinkung mit dem Handlungserfolg

\footnotetext{
${ }^{21}$ Beim psychologischen Vertrag handelt es sich um Erwartungshaltungen (über den Arbeitsvertrag hinausgehend), die die gegenseitige Beziehung von Arbeitnehmer und Arbeitgeber als Bestandteil der Arbeitsbeziehung kennzeichnet.
} 
3.3.1 Qualität heuristischer Entscheidungen

gelingt (siehe Abbildung 4). Diese Gedanken folgen dem Ansatz, dass ein rationaler Entscheidungsprozess in der ökologischen Realität nicht vorkommt, sondern immer durch intuitive oder irrationale Einflüsse der Umwelt und ihrer Akteure/-innen konterkariert wird.

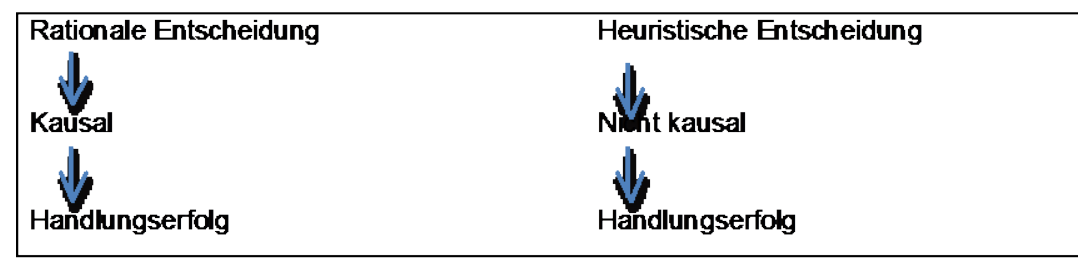

Abbildung 4: Kausale Beziehung zwischen Entscheidung und Handlungserfolg im rationalen bzw. heuristischen Entscheidungsprozess (eigene Darstellung).

Es wird davon ausgegangen, dass eine kausale Ableitung der heuristischen Entscheidung zu einem Handlungserfolg oder -misserfolg in einem naturalistischen Kontext schwerlich gelingen kann.

\section{3. Ökologische Rationalität}

Im Merkmal der Rationalität sollen Kausalität und Angemessenheit zusammengeführt werden. Um das Wirken von Heuristiken im Organisationsgeschehen zu verstehen, wird ein Blick auf die Rationalität des Entschei- 
3.3.1 Qualität heuristischer Entscheidungen

dungshandelns nötig. Die vorliegende Arbeit nimmt Heuristiken sowohl auf individueller als auch auf Gruppenebene als Entscheidungseffekt einer nicht rationalen Entscheidungsfindung an, geht aber nicht (automatisch) von einem Entscheidungsdefekt aus. Der Grund dafür liegt vor allem in der (dargestellten) oft fehlenden Kausalität zwischen heuristischer Entscheidung und Handlungsverfolg. Entgegen den Überlegungen von Simons Theorie begrenzter Rationalität (1959), dessen Annahme es ist, dass optimales Entscheidungsverhalten die erstrebenswerteste Form von Organisationsverhalten sei, stellen Heuristiken ein irrationales Entscheidungsverhalten dar, vor dem sich keine Organisation verschließen kann, da es tagtäglich praktiziert wird. Der Ansatz einer unbegrenzten Rationalität (zur Nutzenmaximierung) wurde im Laufe der Zeit durch die realitätsnahe Betrachtung der begrenzten Rationalität ersetzt. Dieser Ansatz stellt menschliches Entscheiden als irrationale kognitive Täuschung vor (vgl. Gigerenzer/Marewski 2013, S. 231). Auch wenn einige Autoren/-innen zwar nicht mehr von rationalem Verhalten als realistischer Größe ausgehen, unterstellen sie weiterhin ein intendiertes rationales Verhalten (vgl. Neumer 2009, S. 19 f.). Denn Individuen so- 
3.3.1 Qualität heuristischer Entscheidungen

wie Gruppen wünschen sich ein rationales Entscheidungsverhalten in ihrem Tun. Wer möchte nicht gern eine objektive Wahl zwischen verschiedenen Produkten beim Einkauf treffen. Auch Gruppen wünschen sich grundsätzlich eine gemeinsame Entscheidung, die auf der Ratio basiert, ohne dass emotionale oder subjektive Entscheidungselemente (wie Sympathien o.Ä.) eine Rolle spielen. Deshalb kann intendiert rationales Entscheidungsverhalten sowohl auf der individuellen als auch auf der kollektiven Ebene verortet werden. ${ }^{22}$

"Menschen verlassen sich auf eine begrenzte Anzahl von heuristischen Prinzipien, die die komplexe Aufgabe, Wahrscheinlichkeiten zu schätzen und Werte vorauszusagen, vereinfachen. Im Allgemeinen sind diese Heuristiken recht nützlich, aber manchmal führen sie zu schweren und systematischen Fehlern" (Tversky/Kahneman 1974, S. 1024). Diese zentrale Aussage charakterisiert das Heuristics-and-bias-Forschungsprogramm. Der

22 Organisationen und ihre kollektiven Handlungsakteure/-innen versuchen diesen "Rationalitätsschwund" durch organisatorische Gegebenheiten zu begrenzen, indem Prozesse (z.B. durch Verfahrensanweisungen) festgelegt und Spielräume dadurch eingeengt werden. 
3.3.1 Qualität heuristischer Entscheidungen

Fast_and-frugal-Ansatz hingegen beschreibt Heuristiken als eine schnelle und sparsame Verarbeitungsmethode im Hinblick auf Entscheidungen. Im Vergleich zum Heuristics-and-bias-Forschungsprogramm stellen die Vertreter/-innen des Fast-and-frugal-Ansatzes (u.a. Gigerenzer 2000, S. 166 f.; Hoffrage/Reimer 2004, S. 437 f.) weniger auf die Wahrscheinlichkeit von Fehlurteilen bei Heuristiken ab als mehr auf den Nutzen, der sich aus der Anwendung von Heuristiken ergibt. Im Heuristics-and-biasAnsatz werden diese vor allem als unzuverlässige Hilfsmittel dargestellt, die untrennbar mit Fehlern und Verzerrungen verbunden seien (vgl. Powalla 2010, S. 80). Gigerenzer und Todd (1999a, S. 7) unterscheiden zwischen unbegrenzter, begrenzter und ökologischer Rationalität (siehe Abbildung 5). Neben den Ansätzen der begrenzten und unbegrenzten Rationalität fragt der dritte Ansatz, der Ansatz der ökologischen Rationalität, danach, ob heuristisches Entscheidungsverhalten überhaupt als Entscheidungsfehler zu diskutieren sei oder ob heuristisches Verhalten „überwunden“ werden müsse. 


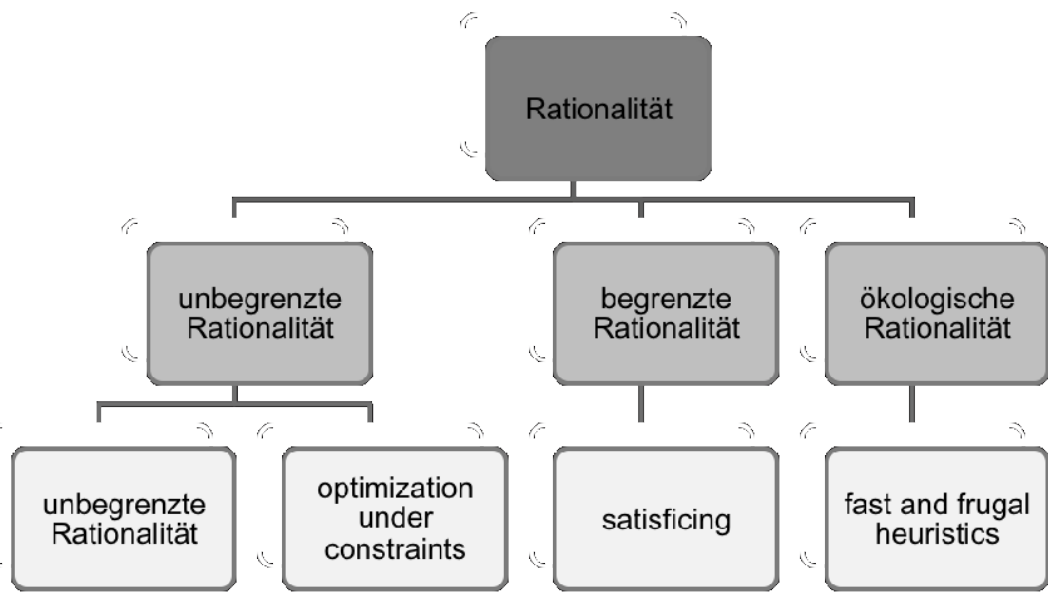

Abbildung 5: Formen der Rationalität, in Anlehnung an: Gigerenzer und Todd (1999, S. 7).

Vielfach wird davon ausgegangen, dass die Ergebnisse heuristischer Entscheidungen gleich gut oder besser seien. Sind die Ergebnisse tatsächlich realistisch, obwohl ihre Erkenntnisse in einer kontrollierten Umgebung gewonnen werden? Die Qualität einer heuristischen Entscheidung wird von den Befürwortern oft als gleichrangig mit anderen Entscheidungshilfen beurteilt: „So konnte nachgewiesen werden, dass Heuristiken komplexe statistische Methoden im Hinblick auf die Genauigkeit von Vorhersagen zukünftiger Ereignisse, Diagnosen und anderen induktiven Inferenzen sogar übertreffen können, obwohl die Heuristiken nur ein Minimum an Informatio- 
3.3.1 Qualität heuristischer Entscheidungen

nen erfordern" (Gigerenzer/Marewski 2013, S. 233). Diese Beurteilung der Heuristiken ruft zunächst einmal Erstaunen und Unglauben hervor.

Der Ansatz der ökologischen Rationalität fragt nicht danach, ob gute Entscheidungen Zufall sind oder ob das Entscheidungsverhalten anfällig für Heuristiken ist, sondern nimmt heuristisches Handeln als deskriptive Beschreibung des Handelns an: "[...] draws on people's natural environments, past and present" (Gigerenzer 2000, S. 57). Heuristiken reduzieren danach lediglich die Komplexität des Handelns in Organisationen, ${ }^{23}$ nicht aber die Qualität der Entscheidung. Ihre Berechtigung ergibt sich bereits aus der menschlichen Natur und hat sogar evolutionsbiologischen Charakter. ${ }^{24}$ Deshalb beziehen sich Heuristiken auf eine bestimmte Umwelt und sind

${ }^{23}$ Dahinter verbergen sich subjektive Entscheidungsregeln, um Entscheidungen in komplexen Situationen unkompliziert und schnell zu treffen (vgl. Neumer 2009, S. 29).

${ }^{24}$ Ein heuristisches Beispiel mit evolutionsbiologischem Charakter ist die Blickheuristik: Um einzuschätzen, ob ein Auto überholt werden kann, unterstützen heuristische Vorgänge die Kognition. Die komplizierte Berechnung der Fahrtgeschwindigkeit des eigenen wie auch des fremden Autos sowie die Umgebungsbetrachtung (Geschwindigkeitsbegrenzung, starker Gegenwind etc.) wird unbewusst ersetzt durch die natürliche Gabe des Individuums, Heuristiken anzuwenden (vgl. Gigerenzer/Gaissmaier 2006, S. 2 f.). 
3.3.1 Qualität heuristischer Entscheidungen

darauf ausgerichtet, eine bestimmte Art von Problemen zu lösen (vgl. Powalla 2010, S. 78). Der Ansatz der ökologischen Rationalität soll damit Aufschluss über die Fragestellung geben, ob Heuristiken Entscheidungshilfen oder Entscheidungsdefekte darstellen. Der Ansatz trennt nicht das "Ehepaar" von "mind und environment" (,two married people who should not be divorced, as often is done" (Gigerenzer 2000, S. 57)). Sie können als Fehlerausgleichsmöglichkeit gewertet werden, die das Entscheidungsverhalten der Organisationsmitglieder prägt (vgl. Auer-Rizzi 1998, S. 160). Heuristiken werden von Vertretern/-innen des Fast-and-frugal-Ansatzes in erster Linie als Entscheidungshilfen für das Entscheidungsverhalten in jedwedem Kontext gesehen, die helfen, der Komplexität vieler Entscheidungssituationen, insbesondere in Organisationen, Herr zu werden.

Müssen gute Entscheidungen auch rational sein? Diese Frage ist nur schwer zu beantworten. Zumindest kann davon ausgegangen werden, dass heuristische Entscheidungen nicht unbedingt schlechte Entscheidungen sein müssen und ein Streben nach einer Entscheidung ohne heuristische Einflüsse ein Paradoxon darstellt, des- 
3.3.1 Qualität heuristischer Entscheidungen

sen Idealbild nur in Laborumgebungen abgebildet werden kann. Heuristische Entscheidungen werden mit dem Ansatz der ökologischen Rationalität aufgewertet und als wertvoll und effizient erachtet. Die Sichtweise der ökologischen Rationalität betrachtet Heuristiken als robuste, suffiziente und schließlich effiziente Entscheidungsregeln, die lediglich in konstruierten, realitätsfremden Situationen zu Fehlschlüssen führen (Gigerenzer/Selten 2001; zit. n. Scholz et al. 2003, S. 16). Außerdem sieht der Ansatz die Gesetze der Logik und der Wahrscheinlichkeitsrechnung nicht als den Bewertungsmaßstab für die Güte menschlichen Denkens. Wenn Entscheidungen von Normen (z.B. Kostenersparnis als Bewertungsnorm oder höherer Umsatz als im Vorjahr) abweichen, werden sie aber meist als defizitär bewertet. Jedoch erst durch eine Abweichung von Normen sind (evolutionsbiologisch) Lernprozesse entstanden, d.h. Lernverhalten, Konditionierung oder ähnliche Mechanismen zur natürlichen Selektion. Sie sind im Laufe der Evolution auch nicht den Gesetzen der Logik gefolgt.

Trotz der Erkenntnis, heuristische Entscheidungen eher als Entscheidungshilfe denn als Defekte zu sehen, stellt 
3.3.1 Qualität heuristischer Entscheidungen

sich in der Tat die Frage, ob Heuristiken eine Form der Reduktion kognitiver Bemühungen darstellen, aber trotzdem als Konsequenz zu schwerwiegenden Entscheidungsfehlern führen (so auch Kahneman/Tversky 1974, S. 1124). Gerade die Natur von Heuristiken, in einem naturalistischen Kontext verwenderfreundlich zu sein, ist vor dem Hintergrund wissenschaftlicher Ergebnisse, die auf Laboruntersuchungen basieren, zu hinterfragen. In einer Laborsituation kann ein Defekt aufgezeichnet worden sein, wohingegen dieser in einem anderen (informationsreichen) Kontext verschwinden kann (vgl. Caverni/Péris 1990; zit. n. Woike 2007, S. 120). Ob Heuristiken eine defizitäre Entscheidung auslösen, hängt auch davon $\mathrm{ab}$, in welcher Umwelt sie wirken. Einen allgemeinen Überblick über die Eigenschaften von Entscheidungen (Informationsverarbeitung, Ressourcennutzung, Ziele und Präferenzen, Informationsaufnahme) in Abhängigkeit von den verschiedenen Ansätzen von Rationalität gibt Tabelle 1. 


\begin{tabular}{|c|c|c|c|}
\hline & $\begin{array}{l}\text { Rationale } \\
\text { Entscheidung }\end{array}$ & $\begin{array}{l}\text { Begrenzte } \\
\text { rationale } \\
\text { Entscheidung }\end{array}$ & $\begin{array}{l}\text { Heuristische } \\
\text { Entscheidung } \\
\text { mit } \\
\text { ökologischer } \\
\text { Rationalität }\end{array}$ \\
\hline $\begin{array}{l}\text { Informa- } \\
\text { tions- } \\
\text { verarbeitung }\end{array}$ & $\begin{array}{l}\text { Vollkommene } \\
\text { Informations- } \\
\text { umgebung }\end{array}$ & $\begin{array}{l}\text { Der Informa- } \\
\text { tions- } \\
\text { beschaffung } \\
\text { sind kognitive } \\
\text { und natürliche } \\
\text { Grenzen ge- } \\
\text { setzt. }\end{array}$ & $\begin{array}{l}\text { Nicht alle In- } \\
\text { formationen } \\
\text { werden be- } \\
\text { rücksichtigt } \\
\text { oder sind vor- } \\
\text { handen. }\end{array}$ \\
\hline $\begin{array}{l}\text { Ressourcen- } \\
\text { nutzung }\end{array}$ & $\begin{array}{l}\text { Unbegrenzte } \\
\text { Ressourcen }\end{array}$ & $\begin{array}{l}\text { Begrenzte } \\
\text { Ressourcen }\end{array}$ & $\begin{array}{l}\text { Nicht alle Res- } \\
\text { sourcen wer- } \\
\text { den genutzt } \\
\text { oder benötigt, } \\
\text { auch wenn sie } \\
\text { vorhanden } \\
\text { sind. }\end{array}$ \\
\hline $\begin{array}{l}\text { Ziele und } \\
\text { Präferenzen }\end{array}$ & $\begin{array}{l}\text { Analytische } \\
\text { Abwägung mit } \\
\text { Zielen zur } \\
\text { Nutzen- } \\
\text { maximierung }\end{array}$ & $\begin{array}{l}\text { Wille zur Nut- } \\
\text { zenoptimie- } \\
\text { rung, aber } \\
\text { durch Unsi- } \\
\text { cherheit der } \\
\text { Umgebung } \\
\text { begrenzt }\end{array}$ & $\begin{array}{l}\text { Ziele sind oft } \\
\text { nicht definiert, } \\
\text { und Präferen- } \\
\text { zen sind un- } \\
\text { klar; Entschei- } \\
\text { dung aus Intui- } \\
\text { tion. }\end{array}$ \\
\hline
\end{tabular}


3.3.1 Qualität heuristischer Entscheidungen

\begin{tabular}{|l|l|l|l|}
\hline Informa- & Lösungssu- & Beenden der & Beenden der \\
tions- & che endet mit & Lösungssuche & Lösungssuche \\
aufnahme & Erreichen des & mit einer zu- & mit einer „an- \\
& Ziels. & friedenstellen- & gepassten“ \\
& & den Option & Option (adap- \\
& & (satisfying) & tive) \\
\hline
\end{tabular}

Tabelle 1: Eigenschaften von Entscheidungen in Abhängigkeit von ihrer Rationalität (eigene Darstellung).

Heuristische Entscheidungen sollen als naturnahe Konstrukte betrachtet werden, die fehleranfällig sind, aber durch ihre positiven Eigenschaften auch eine schnelle priorisierte Entscheidung ermöglichen. Die Wahrscheinlichkeit, dass eine heuristisch getroffene Entscheidung eine Fehlentscheidung ist, ist vorhanden, aber keine zwingende Ableitung oder gar kausal zum Entscheidungsprozess. Beide Ansichten sind vertretbar: Heuristiken als Entscheidungshilfen und brauchbare kognitive Unterstützung für komplexe Probleme auf der einen Seite oder Heuristiken als Entscheidungsdefekte auf der anderen Seite, dessen begrenzte Rationalität für eine fehlerfreie Lösung nicht ausreicht. Sowohl die Angemessenheit als Qualitätskriterium der Entscheidung als auch die hohe ökologische Rationalität geben noch keinen Auf- 
3.3.1 Qualität heuristischer Entscheidungen

schluss über die Beschaffenheit einer heuristischen Entscheidung als Defekt oder Hilfe, sodass diese Frage unbeantwortet bleiben muss. Im Gegenteil, in ihrer Offenheit liegt die Chance, beide Sichtweisen zu akzeptieren und sich nicht blind auf eine zu versteifen und damit Gefahr zu laufen, wichtige Hinweisreize zu übersehen oder Risiken falsch einschätzen. Ein schlüssiges Fazit gibt Gigerenzer (2004, S. 65): Eine Heuristik sei folglich „nicht gut oder schlecht, rational oder irrational per se, sondern relativ zu den vorliegenden Umweltbedingungen“. Außerdem gibt er einschränkend zu bedenken: „The program of ecological rationality is a research heuristic, not a foolproof recipe, just as new laboratory tools do not always lead to good theories for mental processes" (Gigerenzer 2000, S. 58). Wie sich Heuristiken in Bezug auf Entscheidungen darstellen, ist also nicht abschließend zu bestimmen und bedarf weiterer Forschung, aber für Entscheidungsprozesse in Organisationen sind sie aufgrund ihrer ökologischen Natur von großer Relevanz. Erst mit dem Wissen um ihre Existenz kann Entscheidungsverhalten bewusst reflektiert werden und darf nicht von vornherein mit dem Stigma der Irrationalität versehen werden. 
3.3.2 Übertragbarkeit von Heuristiken auf die Gruppenebene

Im Folgenden soll die Frage diskutiert werden, ob Heuristiken auch ein kollektives Organisationsphänomen sein können. Konkret, ob heuristische Entscheidungen auch in Gruppen präsent sind und nicht lediglich die Addition aller Einzelheuristiken einer Gruppe darstellen, sondern ob sie eine gemeinsame oder sich wechselseitig beeinflussende Dynamik haben. Dazu muss zunächst geprüft werden, ob ein Sprung auf die kollektive Ebene gelingen kann. Inwieweit deshalb eine Übertragbarkeit von Heuristiken auf die Gruppenebene möglich ist, soll im nächsten Kapitel diskutiert werden, indem die Annahmen von Gigerenzer und Todd (1999) von der individuellen Verhaltensebene auf die Gruppenebene erweitert werden.

\subsection{2. Übertragbarkeit von Heuristiken auf die Gruppenebene}

Organisationsentscheidungen werden häufig in Gremien, Gruppen oder Teams getroffen. Um Heuristiken konkret auf diese kollektive Ebene mit mehreren Teilnehmenden zu übertragen, sind weitere Vorüberlegungen notwendig. Diese Überlegungen sind u.a. angelehnt an die Analyse 
3.3.2 Übertragbarkeit von Heuristiken auf die Gruppenebene

"Entscheidungsheuristiken in Gruppen“ von Reimer et al. (2007). Im Folgenden sollen die drei Grundregeln der Heuristik (siehe Kapitel 3.3.2) von Gigerenzer und Todd (1999) sowie Gigerenzer und Marewski (2013, S. 234) dargestellt (siehe Tabelle 2) und auf die kollektive Ebene ausgeweitet werden.

Tabelle 2: Grundregeln heuristischer Entscheidungen (eigene Darstellung).

1. Informationen suchen (Suchregel: Wie und in welcher Reihenfolge wird die verfügbare Information verarbeitet?)

2. Beenden der Informationsaufnahme (Stoppregel)

3. Entscheidungsfindung auf Grundlage der vorhandenen Informationen (Entscheidungsregel)

Neben den zahlreichen Einflüssen, die auf den Teilnehmenden sowie der Organisation basieren, gibt es auch einige kollektive Entscheidungsregeln, die eine Rolle bei der heuristischen Entscheidung spielen. Für ein Gruppenmodell werden soziale Komponenten, wie Entscheidungsregeln und Einflüsse, benötigt. Auch Baron et al. 
3.3.2 Übertragbarkeit von Heuristiken auf die Gruppenebene

(1992, S. 94 f.) fragen, ob es reguläre Muster (Entscheidungsregeln) des Gruppenverhaltens gibt, die Präferenzen verändern, sowie ob Gruppenentscheidungen vorhergesagt werden können, wenn man die Präferenzen der Gruppenmitglieder kennt: „It tries to specifiy how group members combine what they bring to the group (e.g. their personal preferences) into a single group product (e.g. group decision)“ (Baron et al. 1992, S. 94).

Jedes Individuum zieht zunächst seine eigenen Schlüsse aus den Entscheidungsalternativen und integriert diese dann in das kollektive Gebaren. Das heißt, jeder/jede hat seine/ihre „eigene“ Heuristik und trifft also eine Vorauswahl für sich selbst. Wenn er/sie in die Gruppe eintritt, „bringt" er/sie diese Vorauswahl mit. Dabei beeinflussen individuelle Präferenzen und Einstellungen das Verhalten anderer Gruppenteilnehmenden (vgl. Baron et. al. 1992, S. 67). Multiple Heuristiken gründen sich nicht nur auf die individuellen Heuristiken, die jedem/jeder Teilnehmenden eigen sind, sondern erst verschiedene soziale Phänomene machen sie zur Heuristik in Gruppen. Die heuristischen Grundregeln von Gigerenzer und Marewski (2013) sollen zum Zwecke der kollektiven Erweiterung auf die 
3.3.2 Übertragbarkeit von Heuristiken auf die Gruppenebene

Gruppenebene durch die Einflüsse „kollektive Entscheidungsregeln“, „Teilnehmendeneinfluss" sowie „Organisationseinfluss" ergänzt werden (siehe Abbildung 6). Einflüsse der Teilnehmenden, der Organisation und kollektive Entscheidungsregeln lenken die Heuristiken der Gruppe und geben ihr Gestalt.
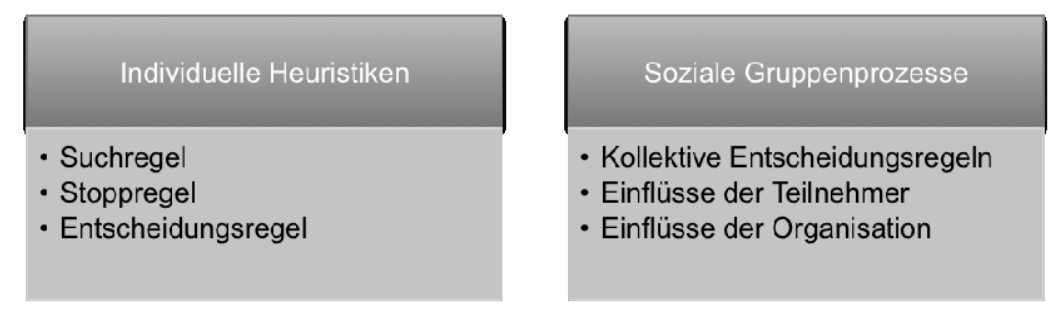

Abbildung 6: Erweiterte Entscheidungsregeln heuristischen Verhaltens durch soziale Prozesse (eigene Darstellung).

Heuristisches Entscheidungsverhalten in Gruppen soll anhand des folgenden Kurzbeispiels (und unter Einbeziehung von Abbildung 6) dargestellt werden:

Beispiel „Personalgremium wählt Azubis für die Organisation aus":

Das Personalgremium der Organisation $X$ hat die Aufgabe, Auszubildende für das neue Lehrjahr einzustellen. Es werden zahlreiche Tests durchgeführt und eine riesige 
3.3.2 Übertragbarkeit von Heuristiken auf die Gruppenebene

Anzahl an Bewerbungsunterlagen gesichtet (Suchregel). Wenn das Bewerbungsverfahren abgeschlossen ist, setzt sich das Gremium zusammen. Es wertet die Unterlagen und Ergebnisse so lange aus, bis die Gremiumsmitglieder der Meinung sind, sie hätten ausreichend Informationen für eine Entscheidung (Stoppregel). Beim Suchprozess des Bewerbungsverfahrens haben sich die Gremiumsmitglieder darauf geeinigt, dass die besten Auszubildenden der Region gefunden werden sollten und die Noten in Mathematik, Deutsch und Englisch eine große Rolle spielen (kollektive Entscheidungsregeln). Zum einen mit der organisationseigenen Begründung, dass diese Auswahlkriterien im Diagnoseprozess der Organisation bei der Auszubildendensuche schon immer wichtig waren (Einfluss der Organisation), und zum anderen, weil die Teilnehmenden im Gremium selbst im Laufe ihrer Organisationskarriere gute Erfahrungen mit den „besten“ Auszubildenden haben gemacht und diese deshalb für zuverlässig und hilfsbereit gehalten werden (Einfluss der Teilnehmenden). 
3.3.2 Übertragbarkeit von Heuristiken auf die Gruppenebene

Heuristiken in Gruppen sind soziale Heuristiken, weil sie erst durch die Zusammenarbeit von mehreren entstehen. Es vollziehen sich dabei Prozesse, die den Entscheidungsverlauf beeinflussen. Heuristiken sind Entscheidungsregeln, wie einfache Mehrheitsregeln (die meisten Stimmen entscheiden) oder Kombinationsregeln, die erst im Zusammenspiel mit den anderen Gruppenmitgliedern ihre Dynamik entfalten (vgl. Reimer et al. 2007, S. 12). Im Unterschied zur einfachen Mehrheitsregel greifen die Kombinationsregeln die Idee auf, dass einige Gruppenmitglieder einen größeren Einfluss haben als andere. Das können z.B. Gruppenmitglieder sein, die eine Rekognitionsheuristik verwenden (vgl. Reimer et al. 2007, S. 12). Aber nicht nur Mehrheitsmeinungen können Entscheidungen beeinflussen, sondern auch Minoritäten können sich durchsetzen, z.B. durch überzeugende Persönlichkeiten oder hierarchische Einflüsse (vgl. Baron et al. 1992, S. 79).

Heuristiken in Gruppen können zwischen heuristischen Mehrheitsregeln (wenn die Mehrheit der Teilnehmenden eine Heuristik anwendet) und wissensbasierten (bzw. 
3.3.2 Übertragbarkeit von Heuristiken auf die Gruppenebene

rationalorientierten) Mehrheitsregeln unterschieden werden, wenn die Mehrheit der Teilnehmenden sich aufgrund von Argumenten entscheidet. Bei wissensbasierten Mehrheitsregeln wägen die Teilnehmenden gemeinsam das Für und Wider einer Entscheidung ab. Sie stützen sich auf greifbare Argumente und Wissen. Informationen werden zurate gezogen und miteinander verglichen. Gruppen entscheiden sich aber häufiger aufgrund einer heuristischen Mehrheitsregel als auf Basis wissensbasierter Mehrheitsregeln, weil auch die Gruppe den "einfacheren" Weg geht, anstatt eine langwierige Prüfung vieler Informationen vorzunehmen, um diese zu rationalen Argumenten (cues) zu formen. Eine Kombination aus beiden Mehrheitsregeln bezeichnen Gaissmaier und Gigerenzer (2006, S. 13) als "heuristisches Denken“ mit cues, d.h., die Gruppenmitglieder verlassen sich nicht nur auf das intuitive Verfahren, z.B. auf das Wiedererkennen von Objekten (Rekognitionsheuristik), sondern nehmen zusätzlich noch die Suche nach Argumenten hinzu. Allerdings schreitet dieses Entscheidungsverfahren nicht so weit voran, dass sich daraus eine rationale Abwägung ergäbe. Sobald der Schwellenwert erreicht ist, d.h. der 
3.3.2 Übertragbarkeit von Heuristiken auf die Gruppenebene

Wert, der die Gruppe entscheiden lässt, dass alle notwenigen Informationen für eine Entscheidung vorhanden sind, wird die Suche abgeschlossen (Stoppregel). Bestimmte Informationen können zu einer sofortigen Eliminierung einer Entscheidungsalternative bzw. eines Lösungsweges führen. Informationen können somit zu einem cut-off der Entscheidungsalternativen führen, wenn eine Information die Kriterien der Entscheidung nicht erfüllt und damit nicht mehr infrage kommt.

Die soziale Dynamik der Gruppe kann einer rein rationalen Entscheidungsregel der Gruppenmehrheit entgegenstehen. Argumente abwägen, sich wirklich für die Besten zu entscheiden anhand von sogenannten cues (Hinweisreizen) und daraufhin eine Abstimmungsauswahl zu treffen, müsste auch zugleich ein Ausblenden anderer sozialer Informationen bedeuten (Sympathie, Persönlichkeit der Redner, Macht etc.). Dieses Verhalten fällt den Teilnehmenden oft schwer, da ihr Handeln durch eigene Prioritäten bestimmt wird, und diese sind nicht immer von einem Abwägen und Vergleichen der Argumente für eine transparente und objektive Entscheidungsfindung ge- 
3.3.2 Übertragbarkeit von Heuristiken auf die Gruppenebene

prägt. Soziale Prozesse zeichnen sich vor allem dadurch aus, dass die menschliche Fähigkeit zum sozialen Lernen und zur Imitation in das heuristische Entscheidungsverhalten integriert wird (vgl. Gigerenzer/Gaissmaier 2006, S. 13).

Die Folge-der-Mehrheit-Heuristik ist eine klassische heuristische Mehrheitsregel. Sie wird auf kollektiver Ebene von den Teilnehmenden angewandt, die sich für das entscheiden, was die Mehrheit möchte. Sie ist eine soziale Heuristik, die auf Wissensfragen in einfachen Urteilssituationen zugeschnitten ist. Wenden die Teilnehmenden eine heuristische Mehrheitsregel an, wählen sie die Entscheidungsalternative aus, für die die Mehrheit der Teilnehmenden abgestimmt hat, die eine Heuristik angewandt haben. Der Unterschied zu wissensbasierten Mehrheitsregeln liegt also in der Anwendung der Heuristiken durch die Gruppenteilnehmenden.

Heuristiken mit kollektivem Hintergrund sind mehr als die Summe des Entscheidungsverhaltens von Individuen. Durch das Agieren in der Gruppe beeinflussen Heuristiken das Entscheidungsverhalten der Gruppe, und auch andersherum werden Heuristiken ihrerseits durch kollek- 
3.3.3 Soziales Verhalten der Gruppe

tive Entscheidungsregeln beeinflusst. Inwieweit sich $\mathrm{Heu}-$ ristiken durch die Gruppendynamik verändern lassen, ist eine interessante Frage. Dieser Versuch sollte an anderer Stelle empirisch unternommen werden; hierzu taugt keine allgemeingültige Modelldarstellung.

So sind die erweiterten Grundregeln der heuristischen Entscheidung ein komplexes Zusammenspiel von sozialen und individuellen Phänomenen. Mit dieser Basis gelingt jedoch der Sprung der Heuristiken von der individuellen auf die kollektive Entscheiderebene, die im Weiteren als Heuristik in Gruppen diskutiert werden soll und insbesondere dem Zustandekommen von heuristischen Entscheidungsverhalten in Gruppen auf Basis des gemeinsamen sozialen Verhaltens von Gruppenmitgliedern dient.

\subsubsection{Soziales Verhalten der Gruppe}

Heuristiken sind zunächst individuelle Phänomene, aber wie wirken sie, wenn sie von Gruppenmitgliedern angewandt werden? Gruppen treffen ihre Entscheidung auch abgekürzt, aber um welche Dynamiken es sich dabei handeln könnte, die ggf. unter dem Oberbegriff „Heuristi- 
3.3.3 Soziales Verhalten der Gruppe

ken in Gruppen" zu subsumieren sind, soll nun untersucht werden. Wie wirkt sich das soziale Verhalten in Bezug auf die Heuristiken in Gruppen aus? Wie entscheiden Gruppen (wie auch Individuen) „aus dem Bauch heraus" oder wie wenden auch sie "Daumenregeln“ an, um schneller zur Problemlösung zu gelangen?

Das Verhalten in Entscheidungssituationen ist oft von Zeitdruck, Wissensüberfluss und hoher inhaltlicher Komplexität (z.B. durch Fachthemen verschiedener Organisationsbereiche) geprägt. All dies spielt der heuristischen Entscheidung von Gruppen in die Hände. Es ist quasi der Nährboden für eine abgekürzte Entscheidung von mehreren Entscheidern/-innen. Heuristische Entscheidungen sind anwendungsorientiert, angemessen und informationsreduziert. Wenn sich die Teilnehmenden zur Beratung und Entscheidungsfindung treffen, wenden sie deswegen genau diese Maßstäbe an. Heuristiken sind daher Anpassungseffekte (vgl. Gigerenzer/Gaissmaier 2006, S. 19), bei denen Organisationsteilnehmende schnell und unbewusst entscheiden. Heuristiken in Gruppen erlauben es trotz begrenzter Ressourcen (Zeit, Energie etc.), zu einer Problemlösung zu gelangen. 
Können auch Gruppen heuristisch entscheiden? Darum soll es im Folgenden gehen. Gemeint sind individuelle Heuristiken, die in Gruppen wirken (nicht eine spezielle Gruppenheuristik). Auch die Bezeichnung Multiple Heuristik von Auer-Rizzi (1998, S. 160) findet für Heuristiken in Gruppen Verwendung. Seines Erachtens seien Gruppen ebenso anfällig für Entscheidungseffekte wie Individuen (a.a.O.). Jedoch führt er seine Überlegungen nur als Grundgedanken ein und geht nicht näher auf sie ein oder definiert sie. Um seine Gedanken auszubauen und das Konzept heuristischer Entscheidungen auf eine kollektive Ebene zu heben, wurden bereits in Kapitel 3.3.2 die Grundkriterien heuristischen Entscheidens (Suchregel, Stoppregel, Entscheidungsregel) um soziale Prozesse, wie kollektive Entscheidungsregeln, Einflüsse der Teilnehmenden und der Organisation, erweitert (siehe Abbildung 7).

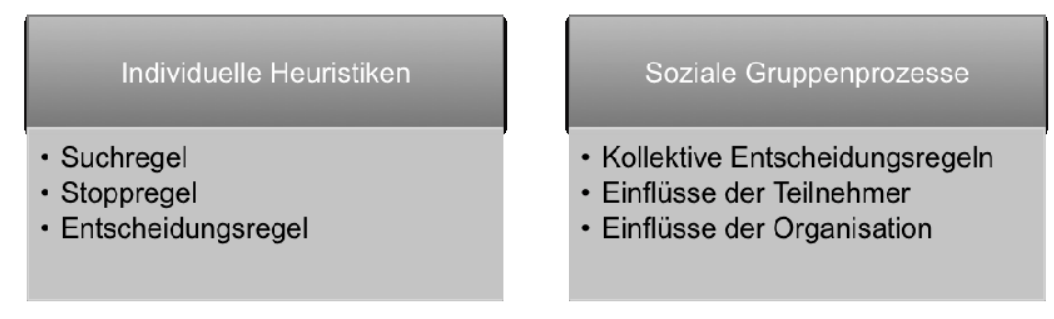

Abbildung 7: Entstehung von Gruppenheuristiken durch soziale Prozesse (eigene Darstellung). 
Die erweiterten Entscheidungsregeln heuristischen Verhaltens sind eine Kombination aus sozialen Gruppenprozessen (z.B. kollektive Entscheidungsregeln durch Group-think) und individuellem Verhalten (Such-, Stoppund Entscheidungsregel). Soziale Gruppenprozesse beeinflussen die Entscheidungen von Einzelnen und können zu einer Veränderung des Entscheidungsverhaltens führen, indem z.B. eigene Präferenzen von Gruppenpräferenzen überlagert werden. Es kann zu einer Inkubation von heuristischem Entscheidungsverhalten kommen: Informationen, die dem Individuum vielleicht wichtig waren, zählen in der Gruppe nicht mehr, oder einzelne Gruppenmitglieder beeinflussen das Entscheidungsverhalten in Richtung der eigenen Präferenzen.

Auch Brandstätter et al. (2010) haben multiple Heuristiken bzw. Heuristiken in Gruppen untersucht. Ihrer Auffassung nach würden Heuristiken in Gruppen angewandt, indem die Gruppenmitglieder sich auf die wichtigsten Informationen und die entscheidendsten Probleme konzentrierten, um nicht einem overload an Wissen und Problemen zu erliegen. Der Gruppenfokus wird ausschließlich auf spezielle Themen und das dafür notwen- 
3.3.3 Soziales Verhalten der Gruppe

dige Wissen gelegt. Ohne Heuristiken würde sich das Handeln der Gruppe verlangsamen und Entscheidungen würden nur zögerlich getroffen werden. Außerdem können für eine gemeinsame Entscheidung Heuristiken richtungsweisend sein, wenn Informationen zusammengetragen werden, Wissen preisgegeben wird oder soziale Effekte die Entscheidung beeinflussen, denn Heuristiken in Gruppen bestehen nicht nur in der Summe der Einzelheuristiken ihrer Mitglieder. Durch das soziale Verhalten der Gruppe entstehen ganz eigene Mechanismen, die die Entscheidungsfindung der Gruppe beeinflussen können. 
3.3.3 Soziales Verhalten der Gruppe

Tabelle 3: Überblick über individuelle Heuristiken und Heuristiken in Gruppen (eigene Darstellung).

\begin{tabular}{|l|l|l|}
\hline & $\begin{array}{l}\text { Individuelle } \\
\text { Heuristiken }\end{array}$ & $\begin{array}{l}\text { Heuristiken in } \\
\text { Gruppen }\end{array}$ \\
\hline Ebene & Individuum & $\begin{array}{l}\text { Mehrere } \\
\text { Teilnehmende }\end{array}$ \\
\hline Kommunikation & $\begin{array}{l}\text { Keine, aber } \\
\text { durchlässig }\end{array}$ & $\begin{array}{l}\text { Durch Austausch, } \\
\text { Abstimmung, } \\
\text { Gruppenbildung etc. }\end{array}$ \\
\hline hnformations- & $\begin{array}{l}\text { Individuell } \\
\text { begrenzt }\end{array}$ & $\begin{array}{l}\text { Informationspooling: } \\
\text { Summe des Wissens } \\
\text { der Teilnehmenden }\end{array}$ \\
\hline Präferenzsystem & Individuell & $\begin{array}{l}\text { Situativ und umwelt- } \\
\text { abhängig, z.B. durch } \\
\text { Präferenzpooling }\end{array}$ \\
\hline
\end{tabular}

Die individuelle Heuristik bewegt sich lediglich im Einflusskreis des Einzelnen, aber sie korrespondiert nicht mit anderen. Bei Heuristiken in Gruppen finden soziale Aspekte Eingang, indem ein Kollektiv von Organisationsteilnehmenden als Gruppe kommuniziert, informiert und 
3.3.3 Soziales Verhalten der Gruppe

sich wechselseitig beeinflusst. ${ }^{25}$ Tabelle 3 zeigt eine Gegenüberstellung der Kriterien von individuellen Heuristiken und Heuristiken in Gruppen. Das Individuum kommuniziert mit seiner Umwelt über den Entscheidungsgegenstand oder das Problem. Heuristische Entscheidungen Einzelner sind deshalb nie isoliert zu betrachten. Der Informationsaustausch des Individuums bei Einzel- oder Mehrpersonenentscheidungen ist durchlässig zu anderen Organisationsteilnehmenden. Durch Kommunikation entsteht bei der Gruppenentscheidung ein (inter- und intragruppaler) Austausch und Entscheidungen können abgestimmt werden.

Die Betrachtungsweise, wonach auch Gruppen Entscheidungen kognitiv abkürzen, soll das Fundament für die weitere Arbeit bilden. Hierzu werden im nächsten Kapitel einige Gruppenphänomene exemplarisch für sozia-

${ }^{25}$ Auch die Gruppenzusammensetzung sowie die Kohäsion von Gruppen können eine Rolle für die sozialen Prozesse spielen: In einer Gruppe mit hoher Kohäsion beeinflussen die Gruppenmitglieder sich gegenseitig anders als in einem „zusammengewürfelten“ Organisationsgremium, das nur gelegentlich oder gar nur einmal zusammentritt. Ihr Verhalten ist weniger kohäsiv, sondern eher zweckmäßig. Erst wenn die Teilnehmenden häufiger gemeinsame Entscheidungen treffen, kann Kohäsion entstehen (vgl. Davis 1969, S. 78). 
les Verhalten in Gruppen angeführt. Insbesondere der Informationsaustausch innerhalb der Gruppe ist für die Heuristik von Gruppen ein zentrales Element.

\subsubsection{Informationsverarbeitung}

Die Erkenntnisse des folgenden Kapitels zur Informationsverarbeitung sind notwendig, um Heuristiken als geistiges und soziales Phänomen einzuordnen. Das Entscheidungsmanagement des menschlichen Geistes ist limitiert durch seine kognitiven Grenzen. Außerdem liegen Entscheidern/-innen in der Realität selten alle notwendigen Informationen vor, um eine Auswahl rational zu treffen. Deshalb stellen die kognitiven Hintergründe heuristischer Informationsverarbeitung sowohl in Gruppen als auch beim Individuum eine interessante Betrachtungsgröße dar.

Menschen verschaffen sich zunächst einen Überblick über ein Problem oder müssen sich erst einmal in die Aufgabe „einarbeiten“, bevor sie ein Urteil fällen (vgl. Behrens 1980, S. 46). Die Aufgabe von Heuristiken ist es dabei, komplexe Prozesse der Problemlösung zu umgehen, in dem die Realität kognitiv vereinfacht wird. Nach 
dem Konzept der beschränkten Rationalität werden Entscheidungen nicht rational getroffen, weil es meist an notwendigen Informationen zur Problemdefinition und der Formulierung relevanter Kriterien und Zielvorstellungen fehlt (vgl. Auer-Rizzi 1998, S. 130). Nur ein Teil der Stimuli aus der Umwelt kann tatsächlich weiterverarbeitet werden (vgl. Sanders/Kianty 2006, S. 172). Dies liegt u.a. an der begrenzten menschlichen Informationsverarbeitungskapazität, die dazu führt, dass nicht alle Alternativen berücksichtigt werden können. Bereits Festinger (1957, S. 5) erkennt die begrenzte Informationsverarbeitungskapazität: „In general, one may say that the preaction or predecision situation will be characterized by extender and non selective seeking of relevant information. [...] Along with the nonselectivity of what information he exposes himself to, there will be a lack of resistance to accepting and cognizing any relevant information which comes his way."

Heuristiken sind also eine kognitive Methode, um schnell und effizient Situationen zu erfassen, wenn nicht alle Informationen verfügbar sind. Aber auch wenn der Zugang vorhanden sein sollte, werden diese oft aufgrund der begrenzten kognitiven Verarbeitungsmöglichkeiten nicht in 
den Entscheidungsprozess integriert. Komplexe Entscheidungssituationen werden somit vereinfacht verarbeitet. Umgangssprachlich werden Heuristiken deshalb auch als Bauchgefühl oder Intuition bezeichnet. Gigerenzer (2007) verwendet auch Faustregel als Bezeichnung für Heuristiken. Auer-Rizzi (1998, S. 130) beschreibt Heuristiken als systematische kognitive Verzerrungen, weil potenziell relevante Informationen vernachlässigt werden. Heuristiken sind deshalb abgekürzte Entscheidungswege, d.h. kognitive Methoden zur Bewältigung von komplexen Vorgängen, die dem kognitiven Gleichgewicht dienen.

Die Informationsverarbeitungskapazität ist auf maximal 7 Zeichen (Gedächtnisspanne) begrenzt (vgl. Behrens 1980 , S. 47). Diese Begrenzung wird durch neurologische Mechanismen ausgeglichen, um trotzdem ein Entscheidungshandeln zu ermöglichen. Entscheidungen werden grundsätzlich unbewusst im limbischen System vorbereitet und sind dort auch schon einige Sekunden vor der Entscheidung lokalisierbar. Oft versucht der Handelnde im Nachhinein dann, seine Entscheidungen mit rationalen Argumenten zu rechtfertigen (Soon et al. 2008; Roth 2003; zit.n. Koppers 2013, S. 56). Auch De Neys 
(2006, S. 428 f.) konnte nachweisen, dass das heuristische System automatisch operiert und die Realität kognitiv vereinfacht. Häufig werden diese Informationen aus dem Kurzzeitgedächtnis abgerufen. Diese aktuelle Wissens-präsenz berücksichtigt deshalb die aktuell vorhandenen eher als die zurückliegenden Informationen, d.h., die Wahrnehmungen und Eindrücke z.B. aus zuvor geführten Gesprächen sind präsenter. Da dieses Wissen über einen längeren Zeitraum nicht präsent gehalten werden kann, kann es unweigerlich nur zu einer heuristischen Informationsverarbeitung kommen. Diese wird noch unterstrichen, wenn eine Gruppe eine gemeinsame Entscheidung trifft und jeder/jede eine andere Wissenspräsenz und -aktualität mitbringt.

Bei der heuristischen Verarbeitung läuft also ein kognitiver Prozess ab, indem die Teilnehmenden auf einfache Regeln vertrauen, um die Gültigkeit von Botschaften zu beurteilen (z.B. „Statistiken lügen nicht"). Diese Regeln sind eng verzahnt mit dem Erfahrungslernen des Individuums (z.B. "Ich konnte mich immer auf das Wissen von Experten/-innen verlassen"). Außerdem werden Entscheidungen unter Berücksichtigung der individuellen Wissenswelt getroffen. Diese „Welt" setzt sich aus indivi- 


\subsubsection{Informationsverarbeitung}

duellen Präferenzen, Einstellungen und Werthaltungen zusammen und gründet sich auf gemachte Erfahrungen und Erlerntes. Die nötigen Informationen für eine Entscheidungsfindung sind nicht immer vorhanden, und daher greift man auf Vorhandenes zurück. Entscheidungen beanspruchen beim Individuum verschiedene kognitive Fähigkeiten (vgl. Behrens 1980, S. 46). Zum Teil werden Informationen erst inkubatorisch verarbeitet oder unbewusst aufgenommen. Um trotzdem zufriedenstellende Lösungen zu erhalten, bedient man sich des kognitiven Tricks der Heuristiken.

Wie verarbeitet nun insbesondere die Gruppe die Informationen, die sie erhält? Die Organisationsteilnehmenden in der Gruppe können nicht alle Informationen sichten, die zu einer rationalen Entscheidungsfindung führen, d.h., auch die Gruppe reduziert das Informationspaket, zunächst jeder/jede Einzelne, dann auch die Gruppe. Ein/eine jeder/jede besinnt sich zunächst auf die ihm/ihr wichtigen Informationen, diese mögen bei jedem/jeder andere sein. Die Informationsreduzierung des Einzelnen hat zunächst den Zweck, das Problem für sich selbst lösbar zu gestalten. Der menschliche Geist greift damit in die biologische Trickkiste und wendet einen Jahrtausen- 
de alten Kniff an: Um den information overload zu vermeiden, wenden mehrere Personen, die ein Problem bearbeiten bzw. eine Auswahl treffen, Informationsverarbeitungs- und Entscheidungsstrategien an, die sowohl an die kognitiven Begrenzungen als auch an die Aufgabenumwelt angepasst sind (vgl. Reimer et al. 2007, S. 8). Heuristiken erfüllen diese Strategievoraussetzungen, indem innen das divergente Denken, d.h. schweifende und springende Denkverläufe (vgl. Rosenstiel/Nerdinger 2011, S. 342) der Entscheidungsträger/-innen zugrunde liegt, die insbesondere durch Kommunikation zutage treten können. ${ }^{26}$ Die Gruppe konzentriert sich dabei auf die Informationen mit der ökologisch rational höchsten Validität auf dem Lösungskriterium (vgl. Lyding 2010, S. 11), d.h. auf die Entscheidungsalternative, die der Gruppe in der Entscheidungssituation als die angemessenste erscheint. Heuristiken können erarbeitet werden, d.h., sie entstehen durch soziales Verhalten z.B. bei einer Diskussion, wodurch informationsreduzierte Entscheidungen von der Gruppe getroffen werden.

${ }^{26}$ Das Gegenteil ist konvergentes Denken (schlussfolgerndes und zielgerichtetes Denken zur Fehlervermeidung), das einen rationaleren Charakter hat. 
Welche Informationen in die Auswahlsituation einfließen und in welcher Reihenfolge dies geschieht, ist dabei von Bedeutung. Durch das Zusammenspiel mit anderen Organisationsmitgliedern kann es zu einer Auf- oder Abwertung von bestimmten Informationen kommen. Informationen werden von jedem/jeder anderes bewertet, wahrgenommen oder erfasst. Mehr noch, Informationen stellen einen Pool dar, der sich von Person zu Person unterscheidet: Ob es sich um ein Investitionspaket handelt, das im Aufsichtsrat diskutiert wird, oder um eine simple Kaufentscheidung, wie den Papiereinkauf der zentralen Einkaufsabteilung, jeder/jede bringt anderes Wissen mit. Dies hängt mit unterschiedlichen Voraussetzungen, wie Bildung, Erfahrung, Spezialisierung, Zugang zu Informationen etc., zusammen. Sogar Expertengruppen lassen sich wegen der spezifischen kognitiven und gruppendynamischen Mechanismen des Informationsaustausches von mehreren beeinflussen und sind dadurch oftmals nicht in der Lage, optimale Entscheidungen zu treffen (vgl. Kerschreiter 2003, S. 92).

Nicht jeder/jede hat also in der Realität die gleichen Informationen. Diese Vielfalt ist für die Rationalität im Ent- 


\subsubsection{Informationsverarbeitung}

scheidungsfindungsprozess positiv zu bewerten und der Grund, warum viele Organisation gemeinsame Entscheidungen begrüßen. Rosenstiel und Nerdinger (2011, S. 343) schreiben Gruppen deshalb eine größere Fähigkeit zu, den Entscheidungsprozess für kreative Lösungen zu nutzen. Die positive Aufladung der Informationsverarbeitung in der Gruppe ergibt sich durch die Informationsvielfalt. Die Gruppe entwickelt gemeinsam neue Alternativen, die sich den Einzelnen nicht erschlossen hätten (vgl. Neumer 2009, S. 13). Dabei besteht die Herausforderung darin, dass „die auf dem Tisch“ liegenden Informationen von der Gruppe gesucht, gesichtet und bewertet werden müssen, bis es zur Entscheidungsreife kommt. Die Gruppe, die die Informationsflut bei Vergleichen, Klassifizierungen oder Schätzungen bewältigen muss, tauscht inr Wissen untereinander aus.

Mit der Teilnahme an sozialen Gruppenprozessen bringen die Gruppenmitglieder ihre Präferenzsysteme in die Gruppendiskussion ein. Die multiple Informationsverarbeitung ist nichts anderes als die Summe aller Präferenzsysteme. Das Pooling von Informationen und Präferenzen ermöglicht die Diskussion in der Gruppe und basiert zumeist auf den individuellen Urteilen der Teilneh- 
menden. Informationspooling versetzt die Gruppe aber nicht automatisch in die Lage, Urteilsverzerrungen auszugleichen. Das Zusammentragen von Gruppenwissen kann aber auch zu einer erhöhten Anfälligkeit für prototypische Informationen führen (vgl. Auer-Rizzi 1998, S. 161) und damit zu einem selektiven Entscheidungsverhalten. Informationspooling durch Kommunikation ist trotzdem das Bindeglied, der die Teilnehmenden von Gruppen eint. Es werden Informationen offengelegt, Kritik geäußert, Konflikte ausgetragen oder Machtpositionen klargestellt. Ihre Ausgestaltungen sind deshalb mannigfaltig und reichern die soziale Dynamik der gemeinsamen Entscheidungsfindung sowohl mit Inhalten als auch mit Hindernissen an.

Reimer et al. (1996, S. 17 f.) haben sich im Hinblick auf Gruppenentscheidungen von Organisationen damit beschäftigt, unter welchen Bedingungen Argumentieren die Problemlösung in der Gruppe verbessert. Nach dem Elaboration Likelihood Model (ELM) von Petty und Cacioppo (1986) stellt Argumentieren eine zentrale Route der Informationsverarbeitung dar. Sympathie, Glaubwürdigkeit, Anzahl und Länge der Argumente können dabei periphere Hinweisreize bei der Informationsverarbeitung darstel- 
len (im ELM ist dies die periphere Route der Informationsverarbeitung) und beeinflussen das Entscheidungsverhalten. Zentrale Hinweisreize stellen z.B. starke Argumente dar, die als Einstellung fest verankert sind. Ihre Untersuchungen ergaben, dass Argumente umso seltener von der Gruppe akzeptiert werden, je mehr sie für falsch gehalten werden. Danach werden die Argumente automatisch gefiltert.

Die Kommunikation der Gruppe ist eine zentrale Komponente des Filters und sie gibt häufig die Marschrichtung vor, d.h., entscheidend sind die Sprachebene, der Sprachduktus und der Gesprächsverlauf. In der Gruppe wird oft bereits Bekanntes wiederholt, was zwar zu einer stärkeren sozialen Bindung der Gruppenmitglieder führen kann, aber nicht zwingend in kontroverse Diskussionen mündet.

Eine Ableitung des ELM ist das heuristischsystematische Modell (HSM) von Chaiken und Eagly (1993), in dem zwischen systematischer und heuristischer Informationsverarbeitung differenziert wird. Das HSM ist wie das Elaboration Likelihood Model (ELM) als ein duales Prozessmodell der Informationsverarbeitung konzipiert. Übereinstimmend nehmen beide Modelle ei- 


\subsubsection{Informationsverarbeitung}

nen systematischen Weg der Informationsverarbeitung (zentraler Weg im ELM) an, indem Einstellungen durch die intensive gedankliche Auseinandersetzung mit einstellungsrelevanten Informationen gebildet oder verändert werden. Sind die Fähigkeiten oder die Motivation zur systematischen Informationsverarbeitung gering, beurteilen Menschen Informationen nach Annahme des HSM unabhängig von den Inhalten mittels einfacher Schemata oder Entscheidungsregeln, sogenannter kognitiver Heuristiken. Die Annahmen des HSM sind in diesem Punkt konkreter als die des ELM, indem die heuristische Informationsverarbeitung nur eine von verschiedenen Strategien der peripheren Informationsverarbeitung ist. Zwar sind beide Modelle der persuasiven Kommunikation und damit dem Erreichen von Einstellungsänderungen zuzuordnen, sodass sie augenscheinlich nicht zum Thema Heuristiken in Gruppen passen. Jedoch soll ausschnittsweise auf die Erkenntnisse des Elementes Informationsverarbeitung abgestellt werden. Denn hinsichtlich des Informationsverarbeitungsprozesses finden sich auch die Unterschiede zwischen den Modellen. Während das ELM die zwei Wege der Informationsverarbeitung als getrennt beschreibt, können nach dem HSM systematische und 
heuristische Informationsverarbeitung gemeinsam auftreten und interagieren. Dieser Ansatz „verträgt" sich mit dem Fast-and-frugal-Ansatz der Heuristikforschung, bei dem das Ignorieren von Informationen als Entscheidungshilfe betrachtet wird, und dies geschieht sowohl durch das Verarbeiten von Informationen, Argumenten und Wissen (systematische Informationsverarbeitung des HSM) als auch durch die heuristische Informationsverarbeitung mittels „simpler“ Entscheidungsregeln.

\subsubsection{Hidden Profile}

Kommunikation, Informationsverhalten und Eigenheiten des Entscheidungsprozesses von Gruppen sind grundlegende Einflüsse auf Heuristiken, die sich auf kollektiver Ebene bewegen sollen. Die Gruppemitglieder führen ihr Wissen zusammen, sie tauschen sich aus und geben damit ihren Präferenzen und Inferenzen Ausdruck. Erst, indem die Gruppe interagiert, entsteht Klarheit über die Meinung einzelner Gruppenmitglieder. Diesem Gedanken folgt das Phänomen des Hidden Profile von Stasser und Titus (1985).

Vielfach ist es Entscheidern/-innen einfach nicht möglich, 
alle Informationen zu erhalten oder zu verwenden, um sich ein vollständiges Bild der Situation zu machen, oder es gestaltet sich zu schwierig, die notwendigen Informationen zu erhalten. Außerdem ist der zeitliche Aufwand oft zu groß für die Aneignung aller relevanten Daten, um eine sichere Abwägung vorzunehmen und sie damit zu objektivieren (vgl. Hinrichs 2011, S. 83). Es stellt sich deshalb die Grundsatzfrage, ob mehr Informationen auch wirklich zu einer besseren Entscheidung führen, ob also ein umfassendes Informationsgerüst zu einer guten oder sogar besseren Entscheidung führt?

Es gibt Informationen, die allen Gruppenmitgliedern bereits bekannt sind, sogenannte geteilte Informationen. Stasser und Titus (1985) konnten herausfinden, dass geteilte Informationen hauptsächlich in die Gruppendiskussion Eingang finden. Solche Informationen, die nur wenigen oder gar nur einem einzigen Gruppenmitglied bekannt sind, werden seltener diskutiert oder aufgebracht (ungeteilte Informationen). Unter den Gruppenmitgliedern werden geteilte Informationen nicht nur häufiger genannt, sondern auch vermehrt akzeptiert (vgl. Kerschreiter 2003, S. 92 f.). Dieses Phänomen wurde als Hidden Pro- 
file bekannt und geht sogar so weit, dass Gruppen nicht in der Lage sind, in einer vorgegebenen Menge von Alternativen die beste Entscheidungsalternative zu identifizieren, wenn diese Alternative nicht bereits vor der Diskussion von mindestens einem Teilnehmenden präferiert wurde (vgl. Reimer et al. 2007, S. 8).

Auch für den kollektiven Personalentscheidungsprozess wird häufig ein vollständiger Informationsaustausch unter den Teilnehmenden als Voraussetzung für gute Entscheidungen genannt, d.h., je mehr Informationen vorliegen, desto ganzheitlicher soll die Entscheidung getroffen werden können. Da Heuristiken diesem Ansatz nicht entsprechen, sondern ein Weniger an Informationen als Ansatz für eine adäquate Entscheidungsfindung annehmen, bietet sich zunächst eine Untersuchung der Informationsverarbeitung innerhalb der Gruppe an.

Reimer et al. (2007, S. 17) geben ein Beispiel für die Informationsverarbeitung der Gruppe anhand des HiddenProfile-Phänomens: Bei zwei Bewerbern/-innen sprechen die meisten Argumente für Kandidatin A. Dies ist aber nicht allen Organisationsteilnehmenden in der Auswahlgruppe bekannt. Eine solche Situation kann entstehen, 
wenn alle die positiven Argumente für B kennen (geteilte Informationen), aber die Informationen, die für A sprechen, nur jeweils einem Gremiumsmitglied bekannt sind (ungeteilte Informationen). Wenn das Gremium alle notwendigen (ungeteilten) Informationen austauscht, würde es sich für Kandidatin $A$ entscheiden, wenn nicht, für Kandidat B. Nur durch den Austausch wird das versteckte Profil (Hidden Profile) der Kandidatin A aufgedeckt. Im Gegensatz zum Konzept der Heuristiken geht das Phänomen der Hidden Profiles davon aus, dass umfassende Informationen wichtig sind für den Entscheidungsprozess und dass keine Informationen verschwiegen werden sollten. Auf Empfehlung der sozialpsychologischen Gruppenforschung sollten die Teilnehmenden möglichst alle verfügbaren Informationen in die Entscheidung integrieren.

Es wird also davon ausgegangen, dass verschwiegenes (hidden) Wissen schädlich für die Entscheidung ist. Für die Personalauswahl könnte dies bedeuten, dass das exklusive Wissen eines Teilnehmenden in der Abschlussdiskussion (z.B. über fehlerhafte Angaben zu den Englischkenntnissen) verschwiegen wird. Der/die Bewerber/-in wird eingestellt und erweist sich als unpassend, 
weil beispielsweise Englischkenntnisse eine zwingende Voraussetzung (z.B. im internationalen Vertrieb) für dessen/deren Aufgabe darstellen. Hierbei führt der HiddenProfile-Effekt dazu, dass entscheidungsrelevante Tatsachen verschwiegen werden, sodass die Entscheidung des Gremiums verzerrt werden kann.

Ein schlechter Informationsaustausch begünstigt den Hidden-Profile-Effekt bei Personalentscheidungen und begrenzt somit die Rationalität des Verfahrens. Hierzu die spiegelbildliche Sichtweise der Fast-and-frugalVertreter/-innen: Heuristiken sind eher ein Weniger-istmehr-Effekt, der nicht als Defekt deklariert wird, da die Berücksichtigung ausgewählter Informationen trotzdem zu guten Entscheidungen führen kann. ${ }^{27}$ Es scheint deshalb so, als ob in dem Beispiel aufgrund des HiddenProfile-Effekts nicht der/die „beste“ Bewerber/-in genommen würde, sondern die anderen, weniger geeigneten Bewerber/-innen. Von den nicht Geeigneten besitzt nicht jeder/jede der Teilnehmenden alle Informationen, wodurch eine verzerrte Gruppenentscheidung produziert

${ }^{27}$ Gigerenzer und Marewski (2013, S. 234) behaupten sogar, dass die Berücksichtigung von weniger Informationen z.B. bei der Takethe-best-Heuristik zu besseren Entscheidungen führe. 
wird. Wobei die Frage gestellt werden muss, ob das Verschweigen von Informationen wirklich einer angemessenen oder organisationsadäquaten Personalentscheidung entgegensteht. Es können auch andere Faktoren und Kompetenzen gleichermaßen relevant für die Stellenentscheidung sein. Wo fehlende Englischkenntnisse als (hidden) Information identifiziert werden, sind Führungskompetenzen u.U. genauso gewichtig oder gar wichtiger für die Stellenanforderungen. Insbesondere wenn es sich um Fachkompetenzen handelt, wird immer häufiger die Meinung vertreten, dass diese als Anforderung in den Hintergrund treten und von der Organisation vermittelt werden können (vgl. Naef 2001, S. 117).

Ein besonderes Wissen, das nur einem Teilnehmenden exklusiv zur Verfügung steht, ist im Bewerberprozess auch zu finden. Die Informationen der Bewerber/-innen liegen in Form von Unterlagen, Testergebnissen, Arbeitsproben etc. vor. Ungeteiltes Wissen kann durch die Wahrnehmung der Gruppenmitglieder entstehen, zu denen die anderen z.B. qua kurze Erfahrung keinen Zugang haben. Die individuelle Wahrnehmung kann zu solchen (wenn auch oft vagen oder subjektiven) Informationen führen. Im Interview kann dem/der einen etwas auffallen, 
was der/die andere übersieht. Hierbei kann es sich um Informationen handeln, die zu ungeteilten Informationen (und damit zu einem Hidden Profile) werden. Widersprüche in der Selbstdarstellung des/der Bewerbers/-in, welche z.B. nur von einem Gremiumsmitglied wahrgenommen werden (z.B. englische Bücher sind dem/der Bewerber/-in zu kompliziert, aber im Lebenslauf hat er/sie Englisch als fließende Sprache angegeben), werden nicht weitergegeben.

Weitere ungeteilte Informationen können z.B. „der Leumund“, persönliche Erfahrungen mit dem/der internen Bewerber/-in o.Ä. sein. Ungeteiltes Wissen kann also eine verschwiegene Information über den/die Bewerber/in sein, welche lediglich dem/der einen Teilnehmenden in der Entscheidungsgruppe bekannt ist und der/die sie (vorläufig) nicht preisgibt. Oder es kann sich um Erfahrungswissen des Teilnehmenden handeln, der eine besondere Expertise in der Personalauswahl hat und diese nicht einbringt. Wissen an andere Teilnehmende preiszugeben, erweitert den Informationshorizont der Gruppe, da das ungeteilte Wissen von einzelnen Experten/-innen in der Personalauswahl auch meinungsbildend für die Gruppe sein kann. Wenn der/die Personalexperte/-in sei- 
ne Informationen nicht teilt, um z.B. organisationspolitische Ränkespiele für seine Zwecke zu nutzen (in der Personalauswahl kann dies häufiger vorkommen, da es sich bei einigen Personaleinstellungen um Entscheidungen mit Tragweite für die Organisation und ihre Teilnehmenden handeln kann) ${ }^{28}$, könnte sich daraus ein verstecktes Profil ergeben. Dadurch entsteht in der Auswahlsituation des Gremiums eine Entscheidungsverzerrung durch das Hidden-Profile-Paradox. Es erschließt sich daher, dass ein Teilnehmender mit besonderer Expertise auch durchaus einen größeren Einfluss auf die anderen Teilnehmenden haben könnte. Wird beispielsweise der Personalauswahlprozess von einer externen Beratungsfirma unterstützt, unterstellt man diesen außerorganisatorischen Teilnehmenden eine besondere Erfahrung, die als „Autoritätsargument" ihrer Meinung mehr Glaubwürdigkeit verleiht. Auch interne Personalverantwortliche können mit einem besonderen Entschei-

${ }^{28}$ Weuster (2012b, S. 56) misst bei der Stimmengewichtung den Entscheidungsträgern/-innen aus der Fachabteilung ein besonderes Gewicht zu, weil sie später mit dem/der ausgewählten Bewerber/-in kooperieren müssen. Dieses mag dann weniger der Fall sein, wenn Spezialkenntnisse und umfangreiche Berufserfahrungen keine Rolle spielen, die von der Fachabteilung geprüft und bewertet werden müssen. 
dungswissen, z.B. durch langjährige Erfahrung als Fachexperten/-innen, durch Auswahlerfahrung als Recruiter/innen oder durch Ausbildung mit psychologischem Hintergrundwissen, ausgestattet sein, sodass auch ihre Stimme ein Autoritätsargument sein kann, d.h. mehr Einfluss hat. Aufgrund der Exklusivität des Expertenwissens kann ihr Wissen als ungeteilt gelten, wenn sie es nicht preisgegeben haben. Dies gilt z.B. für die Beobachtung der Körpersprache: Durch die Fähigkeit eines/einer Experten/Expertin, Körpersprache zu beurteilen, kann ihm/ihr auffallen, dass ein/eine Bewerber/Bewerberin bei der Frage, ob ihm die interne Aufgabe liege, nervös auf dem Stuhl hin- und herrutscht, die Händen ringt, sich an die Nase fasst o.Ä. Durch die Beobachtung des/der Experten/-in kann dann ungeteiltes Wissen entstehen.

Beispielsweise kann auch elitäres Netzwerkwissen um einen/eine Bewerber/-in („den kenne ich aus dem Tennisverein und er/sie ist sehr engagiert") dazu führen, dass bei Einzelnen die vormals abgekürzte Entscheidungsfindung zu einer anderen Entscheidungsalternative umschwenkt. Wenn ein Individuum das soziale Engagement (Tennisverein) als Auswahlkriterium über alle anderen Auswahlkriterien stellt, könnte die neue (geteilte) In- 
formation zu einer Änderung der Heuristik führen. Das hätte zur Folge, dass sich das heuristische Entscheidungsbild des Gremiums insgesamt ändern könnte.

Einen anderen Effekt der Informationsverarbeitung in Gruppen im Rahmen des Hidden-Profile-Effektes kann das Mutual-enhancement-Modell von Wittenbaum, Hubbell und Zuckerman (1999) erklären. Das Modell geht davon aus, dass geteilte Informationen als glaubwürdiger und wichtiger eingeschätzt werden als ungeteilte Informationen, die nur einzelnen Gruppenmitgliedern vorliegen. Die Gruppenmitglieder werten sich gegenseitig auf, indem sie ihre geteilten Informationen gegenseitig sozial validieren. Deshalb entsteht für die Gruppenmitglieder erst der Anreiz, Informationen einzubringen. Die Grundlage hierfür ist, dass der/die Informationsempfangende sowohl sich selbst auch als den Sender als kompetent einschätzt. Die Gruppenmitglieder nehmen einander als kompetent wahr, sodass geteilte Informationen bevorzugt werden, d.h. umgangssprachlich, die Gruppenmitglieder „klopfen sich gegenseitig auf die Schulter", weil sie das gleiche Wissen teilen. Der Informationsgeber mit der Expertise stellt seine besondere Information der Gruppe zur 
Verfügung, er teilt sie. Im Gegensatz zu einem Hidden Profile kommt es hier zu einer subjektiv gefärbten (aber geteilten) Information, die dazu führt, dass Teilnehmende in Richtung einer anderen Heuristik schwenken oder die eigene Vormeinung nicht mehr abwägen.

Für die vorliegende Arbeit hat die Informationsverarbeitung der Gruppe sowohl durch den Mutual-enhancementEffekt als auch durch das Hidden-Profile-Phänomen eine lenkende Wirkung auf die heuristische „Schnelldiagnose“. Sie kann bei der Entscheidungsfindung aber zu einem schnelleren Schwenk in der Meinungsbildung führt. Es soll aber nicht davon ausgegangen, dass die Gruppenentscheidung durch das Hidden-Profile-Phänomen zwingend negativ beeinflusst wird.

\subsubsection{Cognitive Maps}

Heuristiken in Gruppen wurden in den vorherigen Kapiteln beschrieben. Der Sprung zu Heuristiken in Gruppen kann auch gelingen, indem die mentale Seite der kognitiven Abkürzung beleuchtet wird, verbunden mit der Frage, ob es ein kollektives Bewusstsein gibt. Dieses soll durch das Phänomen der Cognitive Maps, der „kognitiven 
Landkarten“, untersucht werden. Gegenstand dieses Kapitels ist deshalb auch die Frage, wie Mitglieder durch die Organisation beeinflusst werden. Entscheidungsmuster wurden bislang nur vor dem Hintergrund des Entscheidungsprozesses im Allgemeinen beleuchtet (siehe Kapitel 2.3.2), jedoch nicht im Zusammenhang mit Heuristiken.

Cognitive Maps sind eine mentale Repräsentation komplexer Zusammenhänge, die innere (dreidimensionale) Landkarten darstellen. Die Landkarten ähneln Räumen der Umgebung. Sie basieren auf individueller Erfahrung. Dabei werden Faktenwissen und andere Informationen, wie eigene Meinungen oder Erfahrungen etc., bildhaft miteinander verknüpft. Individuen verfügen deshalb über sogenannte Bedeutungsschemata, auf die sie zurückgreifen können (vgl. Jochims 2010, S. 60).

Cognitive Maps sind zunächst ein Phänomen, das dem individuellen Geist entspringt, aber sie können auch einen kollektiven Geist haben. Abgeleitet aus der Organisationsstruktur, bilden Cognitive Maps in der Organisation die Repräsentation kollektiver Muster und stellen dabei die Verfestigung von Überzeugungen dar (vgl. Jochims 2010, S. 60). Überzeugungen werden im Weite- 
ren als Einstellungen und Werte verstanden. Sie bilden sich durch Lern- und Veränderungsprozesse, an denen die Organisationsmitglieder teilhaben, indem sie sich an die veränderte Umwelt und an Gelerntes anpassen (vgl. Koppers 2013, S. 31). Dadurch entstehen die kognitiven Schemata oder mentalen Karten der Organisation bei den Organisationsmitgliedern. Sie sind das überlappende Bewusstsein der Gruppenmitglieder und ähnlich einem Netzwerk.

Auch bei den Cognitive Maps handelt es sich um kognitive Vereinfachungsregeln der Informationsverarbeitung, die jedes Individuum anwendet und damit ein individuelles Muster gedankengeografischer Zusammenhänge schafft sowie die räumliche Abbildung von Heuristiken im Geiste. Auch hier wirkt die menschliche Informationsverarbeitung auf die Entscheidungen der Gruppe. Die Wahrnehmungsgeografie von Heuristiken in Gruppen ist ähnlich geprägt, wenn die Teilnehmenden eine ähnliche Vorstellung von der Realität haben. Das heißt, wenn die an einem Entscheidungsprozess Teilnehmenden ähnliche Cognitive Maps haben, werden die Umweltinformationen auch ähnlich gedeutet. „Es gibt in der Organisation demnach so etwas wie ein ,kollektives Bewusstsein"“ 
(collective mind) (Jochims 2010, S. 60). Auch kann die Realität an bestimmten Punkten falsch abgebildet werden, dann können die Cognitive Maps auch zu einer systematischen Fehlerquelle werden (vgl. Martin 1995, S. 27). Die kognitiven Bedeutungsschemata, die eine Art „Bewusstsein“ der Organisation herausbilden, zeigen sich, indem der Bedeutungsgehalt von Informationen und den Informationsbeziehungen abstrahiert, kategorisiert und abgespeichert wird (vgl. Anderson 2001, S. 153, zit. n. Koppers 2013, S. 31).

In Organisationen können Cognitive Maps sowohl von vorgegebenen als auch von nicht vorgegebenen Organisationsstrukturen beeinflusst werden. Die Informationen in der Organisation und die Informationen über sie sind für die Organisationsteilnehmenden abrufbar und bestehen in Merkpunkten und Prozesswissen, das zu Entscheidungen führt (vgl. Jarz 1997, S. 74). In einer Arbeitssituation konstruieren und interpretieren die Teilnehmenden ihre Wirklichkeit in einem kollektiven Prozess. Dieser Prozess vollzieht sich auf Basis bereits vorliegender Wirklichkeitsdefinitionen über die ein mehr oder minder ausgeprägter Konsens besteht. Die räumliche Verknüpfung des Wissens der Organisationsteilnehmen- 
den ähnelt sich, und dieses „Routenwissen“ ist durch eine Abfolge von Merkpunkten miteinander verknüpft (vgl. Jarz 1997, S. 74). Der ganzheitliche, langfristige Charakter des Routenwissens von Organisationen kann als eine Form der Cognitive Maps der Organisation verstanden werden. Die Cognitive Maps der Organisationsteilnehmenden können sich ähneln, nur die individuelle Repräsentativität ist höchst einzigartig. So, wie keine Landschaft der anderen ähnelt, sind die mentalen Räume der einzelnen Organisationsmitglieder auch einzigartig. Sie verbinden sich erst zu einem Gesamtgebilde durch gemeinsame (erlernte) Vorstellungen. Der gemeinsame mentale Raum wird zusammen beschritten, indem bestehende formale und informelle Regeln ähnlich interpretiert werden, sodass es zu einer Annäherung der Sichtweisen kommt. Die Organisationsmitglieder dekodieren die Informationen ähnlich und vervollständigen diese Informationen untereinander (vgl. Koppers 2013, S. 31).

Aus dem kollektiven Bewusstsein resultiert aber nicht automatisch eine deckungsgleiche Heuristik. Ähnliche Cognitive Maps der Organisationsmitglieder können zu ähnlichen Denkmustern führen. Diese Ähnlichkeit wird in gemeinsamen Entscheidungsregeln gebunden. Ein Bei- 
spiel ist dafür die Organisationskultur eines Unternehmens. Gruppenentscheidungen orientieren sich oft unbewusst an der Organisationskultur und interpretieren diese gemeinsam auf eine oft ähnliche Weise. Es ist das Besondere oder Typische einer Organisation, das oft nicht explizit genannt werden kann, aber von den Mitgliedern als Grundannahmen geteilt wird (vgl. Scholl 2004, S. 539). Abgekürzte Denkwege im Sinne von Heuristiken kommen dadurch zustande, sodass diese ähnliche Denkweise ein kollektives Bewusstsein der Organisation schafft. Ist die Organisationskultur z.B. durch den/die Gründer/Gründerin des Unternehmens geprägt, fragt sich die Gruppe - vielleicht unbewusst -, wie dieser/diese entscheiden würde, da zuvor jede Entscheidung über seinen/ihren „Tisch“ ging. Heuristiken stellen intuitive und oft unbewusste Phänomene dar. Mit den Cognitive Maps gelingt es, eine gewisse Ähnlichkeit in den Heuristiken der Gruppe zu konstruieren.

Die Cognitive Maps der Organisation können in den Köpfen der Teilnehmenden ähnlich sein. In der Vorstellung der Organisation sind Entscheidungen der Organisationsteilnehmenden durch einen kollektiven Sinn motiviert. Die Teilnehmenden interpretieren jedoch auf ihre Weise und 
ziehen daraus ihre Schlüsse, die zu Entscheidungen führen können. Ihre Entscheidungen werden stets auch vor dem Hintergrund der kognitiven Landkarten der Organisation getroffen und sind deshalb von heuristischen Einflüssen der Organisation geprägt. Durch die organisationalen Gemeinsamkeiten haben die Organisationsmitglieder ein ähnliches „inneres Bild“ der wahrgenommenen Realität. Ein weiteres Beispiel hierfür sind Unternehmenswerte. Häufig zeichnen sich diese in den Cognitive Maps ab (implizite oder explizite Werte, z.B. anhand des Code of Conduct oder der Einstellungsrichtlinien der Organisation) oder in den Werten der Teilnehmenden (interpretierte oder wahrgenommene Werte, z.B. individuelle Leistung statt Teamwork). Damit nehmen die einzelnen Personen Entscheidungen sowohl vor dem Hintergrund des kollektiven Bewusstseins als auch vor dem Hintergrund ihrer eigenen Cognitive Maps vor. Die gemeinsamen Cognitive Maps der Teilnehmenden bilden die Schnittmenge der organisatorischen Vorstellungen.

Cognitive Maps geben eine einheitliche Vorstellung der Gruppe über die Organisation $a b$, die bei abgekürzten Entscheidungen im Gruppengeschehen eine Rolle spielen können. Das erweitert das herkömmliche Konzept der 
3.4 Beispiele für ausgewählte Defekte in Gruppen

heuristischen Entscheidungen, welches auf individuellen Phänomenen basiert, um die Ebene von Gruppen. Ähnliche Vorstellungen werden in Cognitive Maps repräsentiert und könnten sich auf eine gewisse Einheitlichkeit gründen, bleiben aber dennoch weiterhin innere Modelle Einzelner, die aber durch soziale Dynamiken verbunden sind. Cognitive Maps könnten ein Bindeglied zwischen der individuellen und der multiplen Heuristik darstellen, da im Interaktionssystem des Menschen sowie dem der Organisation Informationen ähnlich wahrgenommen werden können. Sie werden dadurch von abstrakt nach konkret dekodiert. Cognitive Maps als Schemata verknüpfen die Lernprozesse Einzelner mit den Organisationsstrukturen und lassen ein kollektives Bewusstsein entstehen, das in Gruppenheuristiken Ausdruck finden kann.

\subsection{Beispiele für ausgewählte Defekte in Gruppen}

Das soziale Verhalten in Gruppen wurde in Kapitel 3.3.3 anhand der drei Phänomene Informationsverarbeitung, Hidden Profile und Cognitive Maps dargestellt. Wie beschrieben, können aus dem sozialen Verhalten von Gruppen Entscheidungsdefekte entstehen. Diese stehen 
3.4 Beispiele für ausgewählte Defekte in Gruppen

nicht im Gegensatz zu Heuristiken, sondern ergänzen die Sichtweise, dass aus sozialem Verhalten beide Phänomene entstehen können. Es stellt sich auch bei den Defekten die grundsätzliche Frage, ob einige Entscheidungsdefekte auf Gruppenebene entscheidend für die vorliegende Arbeit sind. Die große Anzahl von Entscheidungsdefekten erschwert die Auswahl. Es kommen zahlreiche Phänomene infrage. Um die Argumentation von kollektiven Entscheidungen fruchtbar zu machen, werden Entscheidungsphänomene vorgestellt, die daraufhin untersucht werden, ob sie auf der Ebene von Gruppenentscheidungen in Organisationen wirken können. Dazu sollen individuelle Defekte die Basis für Gruppendefekte bilden (vgl. Ahrens 1976, S. 29).

Falsche Entscheidungen können auch von Gruppen getroffen werden. An Mehrpersonenentscheidungen Teilnehmenden wird häufig unterstellt, dass ihre Entscheidung durch die Vielzahl von Perspektiven und Meinungen eine bessere Entscheidung produziere. Nach dem Motto „Viele Augen sehen mehr als zwei“ scheinen Gruppen geradezu prädestiniert für einen verantwortungsvollen Umgang mit Organisationsentscheidungen. 
3.4 Beispiele für ausgewählte Defekte in Gruppen

Deshalb können Gruppen vermeintlich alle Entscheidungen überlassen werden?

Um dies zu hinterfragen, wurden drei Entscheidungsdefekte ausgewählt: Verantwortungsdiffusion, Kontrollillusion und eskalierendes Commitment. Bei der Kontrollillusion handelt es sich um den Irrtum der Gruppe, alles „im Griff“ zu haben; bei der Verantwortungsdiffusion um die irrtümliche Annahme, dass die Verantwortung für die Entscheidung an die Gruppe abgegeben werden könne bzw. diffundiere; beim eskalierenden Commitment fühlt sich die Gruppe an eine frühere Entscheidung gebunden und investiert deshalb zu viel (das Investment eskaliert), da das Einbringen zusätzlicher Ressourcen ineffektiv ist.

Diese Defekte haben sozialen Charakter und können in Gruppen wirken. Sie wurden als besondere Entscheidungsphänomene für Gruppen deshalb ausgewählt, weil es sich um psychologische Phänomene handelt, bei denen die Entscheider/-innen im Prozess einem Irrtum unterliegen. Sie stellen exemplarische Entscheidungsfehler der Gruppe dar, denn die Entscheidungspraxis von Individuen ist auch immer an kollektive Phänomene gekoppelt, die sozialen Ursprungs sind. 


\subsubsection{Verantwortungsdiffusion}

In einer Gruppe von vielen ist es leicht, sich hinter der Gruppe zu verstecken und auf diese zu verweisen. Das Experiment von Ringelmann (1913) soll dafür den Auftakt zur Erklärung dieses psychologischen DrückebergerPhänomens bilden. Er untersuchte die Arbeit von Menschen in Gruppen und ließ dafür Personen an einem Seil ziehen. Eine Person zog ein $20 \mathrm{~kg}$ Gewicht mit einer Kraft von 63 kg, zwei Personen zogen zusammen 118 kg (also $8 \mathrm{~kg}$ weniger) und drei Personen zusammen $160 \mathrm{~kg}$ (also $20 \mathrm{~kg}$ weniger). Je größer die Gruppe wurde, desto mehr sank die Produktivität. Es handelt sich um eine additive Aufgabe mit physiologischem Hintergrund, die sich als Beispiel nur schwerlich auf heutige, arbeitsteilig organisierte Gruppen zuschneiden lässt. Dennoch verdeutlicht das Beispiel, dass die Leistungsfähigkeit von Gruppen deutlich niedriger ausfallen kann, je mehr Teilnehmende sie hat. Das Experiment zeigt, dass bestimmte Gruppeneffekte (hier: je mehr Personen, desto weniger Anstrengung des Einzelnen) zu einer Veränderung der Leistungsentscheidung von Gruppenmitgliedern führen kann. Nun ein Beispiel für die Verantwortungsdiffusion in Organisationen: Oft ist nicht klar, wer nach einer Ent- 


\subsubsection{Verantwortungsdiffusion}

scheidung dafür verantwortlich ist, ob z.B. der/die eingestellte Bewerber/-in innerhalb der Probezeit weiterbeschäftigt oder freigesetzt wird (vgl. Moser/Kraft 2008, S. 115). Moser und Kraft (a.a.O.) beschreiben drei Geschäftsführer, die eine Person einstellen. Die Einstellungsentscheidung ist getroffen, aber die Überprüfung der Leistung des/der Eingestellten wird von keinem der Geschäftsführer als eigene Aufgabe gesehen. Genauso verhält es sich mit der Trennungsentscheidung, die keiner für sich beansprucht. ${ }^{29}$

Das Beispiel zeigt, dass die Verantwortungsdiffusion sich durch alle Personalprozesse und Verantwortungskreise ziehen kann, die entweder nicht definiert sind, oder Einzelne aus der Gruppe die Aufgabe nicht für sich beanspruchen.

Im Kern geht es um mangelnde Motivation durch die Gruppe. Je mehr Teilnehmende eine Gruppe hat, desto

29 Moser und Kraft (2008) gehen davon aus, dass Verantwortungsdiffusion auch als Einflussgröße für eskalierendes Commitment bezeichnet werden kann. Organisationen trennen sich deshalb eher schwerer von Mitarbeitern/-innen, weil die Einstellungsentscheidung unterstrichen werden soll (der eigene Fehler wird nicht eingestanden) und man deshalb mit immer weiteren Argumenten nach einer Begründung für die Einstellung sucht - bis hin zur Beförderung des/der unqualifizierten Kandidaten/in. 


\subsubsection{Verantwortungsdiffusion}

niedriger kann die Leistung ausfallen. Je größer die Gruppe, desto stärker nimmt das Verantwortungsbewusstsein der einzelnen Gruppenmitglieder ab (vgl. Baron et al. 1992, S. 5). Zurückzuführen ist diese motivatorische Schwächung der einzelnen Gruppenmitglieder auf die Effekte soziales Faulenzen (social loafing) und Trittbrettfahren (free-riding), auch sucker effect genannt (vgl. Drewes et al. 2011, S. 228). Es handelt sich um Effekte, die nur in Gruppen auftreten und alle gemein haben, dass sich einzelne Gruppenmitglieder hinter dem Ergebnis der Gruppe verstecken. Der Motivationsverlust (Individuum) ist zu trennen vom Koordinationsverlust (Gruppe), welcher den interaktiven Charakter der Gruppe zeigt (vgl. Mojzisch 2009, S. 6).

Die Ursachen für ein verringerte Leistungsentscheidung sind eine niedrige persönliche Relevanz und eine niedrige Identifizierbarkeit (vgl. Bierhoff 2000, S. 347). Zwar mögen die Gruppenmitglieder weiterhin motiviert sein, eine gute Entscheidung zu treffen, aber ihr Bemühen ist geschwächt, weil sie keine unmittelbare Verantwortung für die Entscheidung tragen. Dies wird als Diffusion von Verantwortung bezeichnet. Die Gruppe wirkt dabei wie ein Schild, hinter dem sich der/die Einzelne anonym ver- 


\subsubsection{Verantwortungsdiffusion}

bergen kann. Beispielsweise ergaben Untersuchungen (vgl. Bierhoff 2000, S. 370): Je größer eine Gruppe von Restaurantgästen ist, desto weniger Trinkgeld wird gegeben. Gleiches gilt auch für die Hilfe in akuten Notsituationen. Die Hilfsbereitschaft sinkt, je mehr Zeugen anwesend sind. Verantwortungsdiffusion erklärt nicht nur das Verhindern von Hilfeleistungen, sondern es kann auch die sozialen Prozesse erklären, die zu Defekten werden können (vgl. Riesenhuber 2006, S. 151).

Das Phänomen der „Verantwortungsdiffusion“ kann also eine „reduzierte Anstrengung" zur Folge haben. Es stellt sich nun die Frage, was die genaue Erklärung dafür ist, dass sich die Leistungsentscheidung (Motivations- und Koordinationsverlusten) des einzelnen Teilnehmenden ändert, wenn er im Kollektiv agiert?

\section{Identifizierbarkeit der individuellen Leistung}

Ob die Leistung eines/einer jeden in der Gruppe identifizierbar ist, hängt z.B. von der Rechenschaftspflicht ab. Muss sich die Gruppe rechtfertigen, wenn sie sich entschieden hat? Dies ist abhängig von den Gegebenheiten der jeweiligen Organisation. Entscheider/-innen müssen ihre Entscheidung begründen und sind verantwortlich für 


\subsubsection{Verantwortungsdiffusion}

ihre Qualität, gegen mögliche Einwände müssen sie sich nicht verteidigen und auch negative Sanktionen hinnehmen. Sie haben also ein eigenes Interesse an einer guten Problemlösung. Bei Entscheidungen, die in Gruppen gefällt werden, müssen die Gruppenmitglieder häufig wenig Rechenschaft in finanzieller oder arbeitsorganisatorischer Hinsicht ablegen (vgl. Weuster 2012, S. 66). Auch bei der Bewertung ihrer eigenen Leistung, z.B. als Fachvorgesetzte oder Personaler/-in spielt das Entscheidungsverhalten eine Rolle.

\section{Persönliche Involviertheit}

Jedes Gruppenmitglied ist zunächst stark involviert, weil von jedem/jeder oft eine Einschätzung verlangt wird bzw. aktives Tun im Sinne von Fragen, Bewertungen oder Darstellungen. Das persönliche Entscheidungsrisiko besteht darin, entweder die Folgen einer Fehlentscheidung tragen zu müssen, sich für eine schlechte Entscheidung rechtfertigen zu müssen, den eigenen Ansprüchen nicht zu genügen etc. Deshalb können die Gruppenmitglieder im Entscheidungsprozess zunächst involviert und motiviert sein, eine angemessene Eignungsdiagnose und Auswahl vorzunehmen. Die persönliche Involviertheit 


\subsubsection{Verantwortungsdiffusion}

kann sich auch aus den Folgen einer Fehlentscheidung ergeben. Als Beispiel muss bei einer unpassenden Personalauswahl, der/die unpassende Kandidat/-in gekündigt, versetzt oder entwickelt werden, und die Personalsuche geht weiter. Bei mehreren Fehlentscheidungen würde auch der „Ruf“ der Gruppe leiden, sodass die persönliche Involviertheit auch auf Gruppenebene anzutreffen ist. Die Zugehörigkeit zu einer Gruppe kann dabei zu einer Veränderung des Verantwortungsbewusstseins führen (vgl. Grünig/Kühn 2012, S. 229). Dies ist das Phänomen der diffusen Verantwortlichkeit, d.h., die Verantwortung für die eigene Leistung nimmt ab. Die Leistung verringert sich umso mehr, je weniger die Person involviert ist, d.h., ihr persönliches Entscheidungsrisiko ist niedrig.

Eine unklare Verantwortungsaufteilung kann bei den Gruppenmitgliedern dazu führen, dass sie die Anonymität nutzen, um in der Gruppe unterzutauchen. Ihre Meinung wird im Strudel der Gruppendynamik „verwässert". Sie verschwindet aber nicht in Gänze wie bei anderen Phänomenen, sondern wird nur überdeckt. Dieses tritt sowohl innerhalb des Kernentscheidungsprozesses („Ich entscheide das nicht allein, sondern stelle mich hinter die 


\subsubsection{Verantwortungsdiffusion}

Gruppe“) als auch danach auf („Ich war eine/r von vielen, der/die diese Entscheidung traf") ${ }^{30}$. Das sich hieraus ergebende Verantwortungsbewusstsein diffundiert und führt zu einer Schwächung der Anstrengungen, die richtige Entscheidung zu finden.

Bei Gruppenentscheidungen kann sich Verantwortungsdiffusion z.B. in mehreren Punkten zeigen:

- Aufgrund der Verantwortungsdiffusion kann es dazu kommen, dass Entscheidungen hinausgezögert werden, weil sich niemand verantwortlich fühlt. Niemand ergreift die Initiative.

- Je mehr Personen mitentscheiden, desto weniger Verantwortung trägt die einzelne Person. Die Gesamtverantwortung diffundiert, weil die "Last" der Verantwortung als kleiner wahrgenommen wird (vgl. Riesenhuber 2006, S. 151). Die Verantwor-

${ }^{30}$ Bei der anschließenden Bewertung der Entscheidung vor dem Hintergrund der Verantwortungsdiffusion kann sich auch eine kognitive Dissonanz zeigen: Eine einmal getroffene Entscheidung, die sich anschließend als ungünstig herausstellt, wird aufgewertet. Der kognitive Dissonanzzustand kann z.B. mit der Begründung „vertrieben“ werden, dass die Gruppe so entschieden habe und der eigene Einfluss gering gewesen sei. Denn genauso wie Individuen können sich auch Gruppen vor Entscheidungen in Konflikten befinden. Diese divergierenden Kognitionen können Interessenskonflikte auslösen (vgl. Ahrens 1976, S. 29). 


\subsubsection{Verantwortungsdiffusion}

tungsdiffusion stellt einen Entscheidungsdefekt dar, der zu einer geringen Motivation führen kann. Die Gemeinschaft hat die Entscheidung gemeinsam getroffen, gefühlt liegt die Verantwortung aber im anonymen, „nicht zu greifenden“ Team. Dieses Verhalten liegt in der Natur von Kollektiven. Dies kann bei einzelnen Entscheidern/-innen zu einer Schwächung seiner/ihrer Anstrengungen, eine richtige Entscheidung zu treffen, führen (vgl. Weuster 2012b, S. 66). Die Konsequenzen sind ein reduziertes Engagement, z.B. bei der Beteiligung an Diskussionen.

- Anonymitätseffekte der Verantwortungsdiffusion treten sowohl während des Entscheidungsprozesses als auch danach zutage, etwa wenn sich die Entscheidung als unbefriedigend herausstellt, und „bietet" der Gruppe nach der „schlechten“ Entscheidung die Möglichkeit, sich „zu verstecken“. Die Ursachen liegen sowohl in der Vermeidung von kognitiven Dissonanzen als auch in dem Aspekt, dass negative Folgen einer Entscheidung eher extern als intern attribuiert werden. 


\subsubsection{Kontrollillusion}

Die Veränderung des Verantwortungsbewusstseins der einzelnen Gruppenmitglieder fußt auf einem Effekt, bei dem sich der/die Einzelne hinter dem anonymen Schild der Gruppe verstecken kann. Die Gruppensituation führt letztlich zu einem eingeschränkten Gefühl der Beteiligung (vgl. Kühn/Grünig 2012, S. 229), da man sich nicht mehr alleinverantwortlich für die Aufgabe der Gruppe fühlt. Die Entscheidungssituation führt zu einer diffusen Verantwortlichkeit.

\subsubsection{Kontrollillusion}

Den Wunsch, „Herr des eigenen Schicksal“ zu sein, teilen auch die Mitglieder von Gruppen. Dabei ist gerade das Schicksal eine nicht berechenbare Wahrscheinlichkeitsgröße. Die Wahrscheinlichkeit, Prozesse und ihre Folgen stärker beeinflussen zu können, als es tatsächlich der Fall ist, ist ein Fallstrick, dem auch die Gruppe unterliegen kann. Zunächst soll der Entscheidungsdefekt Kontrollillusion vorgestellt werden, um ihm anschließend auf Gruppenebene nachzugehen.

Bei der Kontrollillusion handelt es sich auch um ein Defizit im Kernentscheidungsprozess (siehe Kapitel 2.3.2), 


\subsubsection{Kontrollillusion}

bei dem sich der Handelnde in der Entscheidungssituation überschätzt und glaubt, Zufallsereignisse beeinflussen zu können, obwohl sein objektiver Einfluss als eher gering einzustufen ist. Zwei Alltagsbeispiele:

- Durch intensives Schütteln der Würfel glauben Spieler, ihre Chancen zu erhöhen.

- Viele Menschen denken, sie seien bessere Autofahrer als alle anderen, und vertrauen deshalb ihrer eigenen Fahrweise mehr als der anderer.

- Obwohl bekannt ist, dass der Flugverkehr als sicher gilt, wird das subjektive Risiko überschätzt.

Diese weit verbreiteten Entscheidungsfehler täuschen die Illusion vor, aktiv Einfluss auf den Zufall nehmen zu können. Es handelt sich demnach um eine subjektiv empfundene Kontrolle. Sie wird deshalb auch als „kognizierte Kontrolle" bezeichnet, weil die tatsächliche Kontrolle nicht relevant ist (vgl. Skinner 1996, S. 552).

Die Kontrollillusion setzt sich aus den drei Elementen Illusion, Kontrolle und positive Einschätzung der Erfolgsaussichten zusammen: 


\subsubsection{Kontrollillusion}

\section{Die Illusion}

Die Illusion ist abhängig von einer falsch eingeschätzten Situation oder des Eigenbildes. Diese Fehleinschätzung können sein: Überschätzung der eigenen Fähigkeiten, Informationsverfügbarkeit, Beeinflussung anderer Personen (vgl. Jungermann 2009, S. 19). Aus der Überschätzung bzw. der Fehleinschätzung resultiert die Handlungsmotivation. Die Binsenweisheit "Glaube versetzt bekanntlich Berge" ist auch hier Credo des Entscheidungshandelns. Die „Berge“ werden durch den „Glauben“ an die besondere wahrgenommene Verfügbarkeit von (Eigen-)Ressourcen versetzt. Die Illusion stellt die Eigenmotivation der Mitglieder dar, weil sie daran glauben, über mehr Kompetenz oder Informationen zu verfügen als andere.

\section{Die Kontrolle}

Das Element der Kontrolle ist die kognitive Seite der Kontrollillusion. Es führt zu einer Verzerrung, weil der/die Entscheider/-in denkt, dass alles seiner/ihrer tatsächlichen Handlungskontrolle unterliege. Dieser Einfluss liegt aber faktisch nicht vor, weil die Wahrscheinlichkeit bei Zufallsaktivitäten nicht beeinflusst werden kann. 


\section{Die positive Einschätzung der Erfolgsaussichten}

Die Verbindung von Illusion und Kontrolle bedarf aber noch eines dritten Elementes, das unabdingbare Voraussetzung für die Kontrollillusion ist: der positiven Einschätzung der Erfolgsaussichten. Der/die Entscheider/-in schätzt das Ergebnis des Entscheidungshandelns als Erfolg ein. Die Konsequenzen sind dann entweder die Herbeiführung des erwünschten Zustandes oder die Vermeidung unerwünschter Ziele.

Fehlerhaftes Entscheidungshandeln des eskalierenden Commitments

\begin{tabular}{llll} 
& \multicolumn{1}{c}{ Beispiel: } & \\
I. & Entscheidung & $\rightarrow$ Ladenkauf (a) & gleichbleibendes \\
II. & Information & $\rightarrow$ Sinkender Umsatz (b) & Commitment \\
III. & Erneute Investition & $\rightarrow$ Neue Innenausstattung (c) & in allen Phasen
\end{tabular}

$\rightarrow$ Versäumnis rechtszeitiger Ausstieg/Kein Abbruch der Missentscheidung (d)

Abbildung 8: Kontrollillusion (eigene Darstellung).

Fehlerhaftes Entscheidungshandeln im Sinne einer Kontrollillusion kommt demnach durch das Zusammenwirken der drei Elemente zustande (siehe Abbildung 8). Zum Beispiel, wenn der/die Entscheider/-in seine/ihre Fähigkeiten überschätzt, sein/ihr Schicksal beeinflussen zu 


\subsubsection{Kontrollillusion}

können, ergibt sich nicht zwangsläufig eine kontrollillusorische Handlung. Denn nur die Kombination aus dem Bewusstsein, eine Situation „im Griff“ zu haben (Illusion), der Neigung, sich zu überschätzen (Kontrolle), und der positiven Einschätzung der Erfolgsaussichten ergeben die irrtümliche Annahme im Sinne einer Kontrollillusion. Oder anders ausgedrückt: Die Überzeugung des Kontrolleinflusses und die Überschätzung der eigenen Fähigkeiten sowie die positive Einschätzung der Konsequenzen wirkt auf das Entscheidungshandeln als Kontrollillusion.

Es ist eine weitverbreitete Art der Täuschung, dass Menschen denken, aktiv Einfluss auf den Zufall haben. Gelten die grundlegenden Voraussetzungen der Kontrollillusion auch für ein Handeln in der Gruppe? Grundsätzlich stellt die Kontrollillusion ein individuelles Phänomen dar, aber gibt es den Entscheidungsfehler der Kontrollillusion auch auf kollektiver Ebene?

Skinner (1996, S. 549 f.) erweitert die Kontrollillusion, indem sie den/die Kontrollagenten/-in als handelnde Person einführt. Der/die Kontrollagent/-in kann sowohl der/die Handelnde, eine andere Person oder eine Gruppe sein. Handeln im Sinne der Kontrollillusion der Gruppe 


\subsubsection{Kontrollillusion}

könnte sich u.a. aus dem sozialen Beziehungsverhalten in der Entscheidungssituation herleiten lassen. ${ }^{31}$

Die soziale Dynamik einer Kontrollillusion könnte sich z.B. durch soziale Prozesse, wie Abgrenzung von anderen Gruppen, Selbstüberschätzung etc., ergeben. HAuch bei einfachen Aufgabenstellungen ist davon auszugehen, dass Gruppendynamiken eine Herausforderung für alle Gruppenmitglieder darstellen. Da zahlreiche Einflüsse, die unabhängig von der Aufgabe sind, eine Rolle spielen, z.B. das Berücksichtigen von langfristigen Konsequenzen der Entscheidung für die Organisation, oder einzelne Mitglieder versuchen sich durchzusetzen. Einzelne Gruppenmitglieder könnten dazu neigen, ihre Fähigkeiten zu überschätzen, z.B. durch die Annahme, alle Gruppenmitglieder zu kennen oder gar Einfluss auf sie bzw. alle notwendigen Informationen $\mathrm{zu}$ haben, da soziale Prozesse komplex und wenig kontrollierbar sind. Es handelt sich dabei um einen selbstregulatorischen Effekt, den die Gruppe unbewusst anwendet, um ihre Umge-

${ }^{31}$ Gerlach (2008, S. 78) geht von einem Zusammenhang zwischen der Komplexität eines bestimmten Entscheidungsproblems von Gruppen (Beispiel: Nachhaltigkeitsinnovation) und einer erhöhten Wahrscheinlichkeit der Neigung zu Kontrollillusionen aus. 


\subsubsection{Kontrollillusion}

bung kontrollierbar zu halten (trotz Stress, Unsicherheit etc.).

Auch das Element "Kontrolle“ ist auf Gruppenebene darstellbar: Die Gruppenmitglieder glauben, die Kontrolle über die Entscheidungssituation zu haben. Die Ursache liegt in dem Streben nach kollektiver Selbstbestimmung. Nur wenn die Gruppe glaubt, alles zu kontrollieren, hat sie die Motivation, welche die Kontrollillusion auslöst. Das Gegenteil wäre, wenn die Gruppe meinte, die Entscheidungsparameter nicht beeinflussen zu können (z.B., weil die Organisation keinen Spielraum bei Entscheidungen lässt oder weil die Entscheidung stark standardisiert ist), und wenig Handlungsraum sähe; dann litte auch die Motivation, sich einzubringen.

Die Überzeugung der Gruppe, alles „im Griff“ zu haben, wird noch durch das dritte Element, die positive Einschätzung der Erfolgsaussichten, ergänzt, ohne die die Kontrollillusion nicht ihre Wirkung entfalten könnte. Auch die Gruppenmitglieder müssen von einer positiven Aussicht auf den Erfolg ihres Handelns ausgehen. Damit könnte der illusorische Aspekt der Selbstüberschätzung bzw. der Fehleinschätzung der Gruppe auch auf Gruppenebene gegeben sein. Der Selbstüberschätzungseffekt 
in der Kontrollillusion wirkt auch als Schutzmantel für die Gruppe. Durch Selbstüberschätzung gelingt es der Gruppe, Gefahren zu untertreiben, zu überspielen oder sich stark auf die Gewinne zu konzentrieren und alles andere auszublenden. Das kollektive Selbstbild führt zu einer falschen Selbstbestätigung.

Die Kontrollillusion birgt für die Organisation mehrere Gefahren (vgl. Peterrein 2008, S. 24):

- Expertenwissen anzweifeln/überhören (Autoritätsargumente)

Die Gruppe stellt bei der Kontrollillusion das eigene Wissen über das von Experten/-innen. Die Selbstüberschätzung kann blind machen für allgemeingültige Erkenntnisse und Organisationswissen.

\section{- Zu viel Aktivität:}

Die positive Einschätzung der Erfolgsaussichten kann dazu führen, dass sie durch vermehrte Aktivität mehr Erfolg erzielen wollen, d.h., sie handeln mehr, als für die Entscheidungssituation notwendig wäre. 


\section{- In Sicherheit wiegen:}

Die Kontrollillusion verschafft auch der Gruppe eine illusorische Sicherheit, alles im Griff zu haben. Durch diese Täuschung können Gefahren unterschätzt werden, und es wird versäumt, den Risiken vorzubeugen oder sie durch ein vorsorgliches Risikomanagement zu minimieren.

Mögliche Ursachen der Kontrollillusion als Gruppenphänomen können sich u.a. aus Folgendem ergeben: Die illusorische Fehleinschätzung der Gruppe kann sich darauf beziehen, u.a. besser, schneller, gezielter als andere Gruppen zu sein oder mehr Erfahrung und Kompetenz sowie eine hohe Identifikation mit der Aufgabe zu haben. Betrachtet man die Gruppe als Makroakteur, kann auch sie ihre Fähigkeiten überschätzen. Ihre gemeinsame Aufgabe verbindet die Gruppenmitglieder und schafft das Gefühl der Kontrollillusion. Die Folge ist ein übertriebenes Selbstbewusstsein, weil die Gruppe an sich glaubt und (irrtümlich) auf ihre Fähigkeiten vertraut. Wenn die Gruppe der Kontrollillusion unterliegt, kann davon ausgegangen werden, dass ihre Entscheidung defizitär ist. 


\subsubsection{Eskalierendes Commitment}

Das Sprichwort „Sehenden Auges in sein Unglück“ rennen, passt zur Entscheidungssituation des eskalierenden Commitments. Die Entscheidung wurde bereits getroffen (Entschluss in der Kernphase, siehe Kapitel 2.3.2), aber der Defekt in der Entscheidung vollzieht sich erst im weiteren Prozessgeschehen und zählt zu den Ex-postElementen des Entscheidungsprozesses. Er lässt sich in den Entscheidungsphasen der Umsetzung und Reflexion finden.

Ein Beispiel für eskalierendes Commitment: Eine Unternehmerin trifft die Grundsatzentscheidung, ein Ladengeschäft zu kaufen (a). Trotz sinkender Umsätze (b) investiert sie weiter, u.a. in eine neue Innenausstattung (c). Obwohl das Geschäft immer unrentabler wird, will sie den Laden behalten (d). Ihre beständige Bindung an die Entscheidung führt dazu, dass ein rechtzeitiger Ausstieg versäumt wird und der Verlust sich dadurch vergrößert. Dies zeigt sich verstärkt, indem sie nicht nur an der Ent- 


\subsubsection{Eskalierendes Commitment}

scheidung festhält, sondern sogar weiter investiert. ${ }^{32}$ Nachdem der erste Entschluss gefasst ist, wird - nach weiteren Misserfolgen - weiterhin an der Entscheidung festgehalten, d.h., das Commitment in den Entscheidungsphasen ist konstant (vgl. Auer-Rizzi 1998, S. 256).

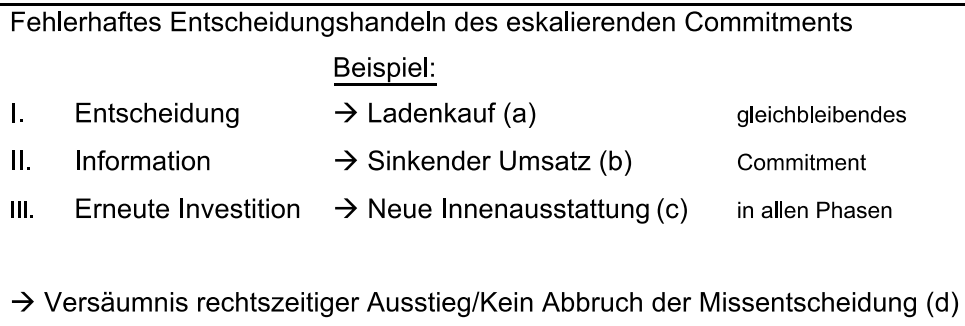

Abbildung 9: Elemente des eskalierenden Commitments (eigene Darstellung).

Beim eskalierenden Commitment erhält der/die Organisationsteilnehmende nach dem Entschluss eine neue Information, bzw. die Situation verändert sich. Die Änderung der Situation sollte eigentlich dazu führen, dass das Commitment zur einstmals getroffenen Entscheidung sinkt. Die Verpflichtung, zu einer Entscheidung zu ste-

${ }^{32}$ Damit ist jedoch nicht eine taktische Eskalation im Sinne eines "Schachzuges“ gemeint (vgl. Martin 2012, S. 10). Bei dieser wäre ein Festhalten an einer verlustreichen Entscheidung eine bewusste Wahl und sie diente strategischen Zielen. 


\subsubsection{Eskalierendes Commitment}

hen, bleibt erhalten, obwohl die Folgen der Entscheidung im Laufe der Umsetzung- und Reflexionsphase sich als negativ herausstellten. Anders ausgedrückt: Eine Entscheidung wird zu einem bestimmten Zeitpunkt getroffen, im Laufe der Reflexionsphase stellt sich aber durch neue entscheidungsrelevante Informationen (schleichend) heraus, dass die Entscheidung in einem Misserfolg enden könnte, der/die Entscheidende aber trotzdem an seiner/ihrer Ursprungsentscheidung festhält. Er/sie wechselt von einer rationalen zu einer emotionalen Situationsbewertung (vgl. Auer-Rizzi 1998, S. 257), weshalb er/sie an der Entscheidung festhält und nicht aussteigt.

Doch warum wird an der Entscheidung festgehalten, obwohl die Situation aus der Perspektive eines objektiven Dritten aussichtslos erscheint? Und warum sollte ein Organisationsteilnehmender an einer "Missinvestition“ festhalten, obwohl er ahnt, dass seine Entscheidung falsch sein könnte? Welche psychologischen Mechanismen stecken also hinter der Nibelungentreue des Entscheidenden zu seiner Entscheidung? Hierzu gibt das Modell des eskalierenden Commitments Erklärungsansätze und es beschreibt die psychologischen Elemente des Ent- 


\subsubsection{Eskalierendes Commitment}

scheidungsdefektes. Neben den drei Elementen des es-

kalierenden Commitments gibt es auch Erklärungsansätze zum psychologischen Ursprung des Entscheidungsdefektes. Die Gründe liegen zum Teil in den Personen selbst, aber auch in den sozialen Beziehungsstrukturen (vgl. Auer-Rizzi 1998, S. 257).

Das Phänomen des eskalierenden Commitments wurde bereits in zahlreichen Feldstudien (u.a. Staw/Ross 1987; Riesenhuber 2006) validiert. Diese Untersuchungen verfolgten das Ziel, Entscheidungsfehler im Verhaltensprozess des Individuums zu analysieren, nicht jedoch in Bezug auf das Gruppenverhalten. Eskalierendes Commitment wird als individueller Entscheidungsdefekt beschrieben, aber kann eskalierendes Commitment auch auf kollektiver Ebene in Gruppen vorkommen? Halten Gruppen nicht auch an unrentablen Entscheidungen fest bzw. investieren sie nicht weiter in fruchtlose Entscheidungen?

Auch in Gruppenentscheidungen können soziale Kräfte mitwirken, die dazu führen, dass sich die Gruppe in besonderem Maße an eine Entscheidung gebunden fühlt. Doch wie kommt es dazu? 


\subsubsection{Eskalierendes Commitment}

Das soziale Netz, also die Organisation und ihre Umwelt, tragen dazu bei, dass die Gruppe unter einem nicht unerheblichen Leistungsdruck steht. Die Organisation (durch ihre Stellvertretenden, z.B. die Geschäftsführung) erwartet von der Gruppe, dass die Organisationsziele zufriedenstellend erfüllt werden. Die Gruppe ist dabei häufig einem starken Rechtfertigungsdruck ausgesetzt. Beispiele sind die Einhaltung von Fristen, die Übernahme von Projektverantwortung, die Beachtung von Budgets etc. Aber auch die Gruppe hat ein Bild von sich, das von den kollektiven Erwartungen und Vorstellungen der Mitglieder geprägt ist. Oft handelt es sich um ein eher inkonsistentes Bild, das das Selbstbild der Gruppe ausmacht. Dieses kollektive Selbstbild der Gruppe prägt deren Handlungsweise.

Beispiel: Eine Gruppe wird beauftragt, ein Bauvorhaben durchzuführen. Dabei ist das Gruppenbild von den jeweiligen Erwartungen, Ansprüchen, Werthaltungen der Gruppenmitglieder geprägt. Das Ziel ist klar formuliert (einen konkreten Bau zu planen und in einer bestimmten Zeit mit einem bestimmten Budget abzuschließen), und die Aufgabenverteilung erscheint durch die Kompetenzen (Ingenieur, Controlling, Personal, Projektplanung etc.) 


\subsubsection{Eskalierendes Commitment}

logisch. Diese vordergründige Aufgabenstellung prägt die Handlungsweise. Die Gruppe möchte vielleicht in der Öffentlichkeit als kompetent und zielstrebig wahrgenommen werden (Reputation der Gruppe). Für eine andere Gruppe oder andere Gruppenmitglieder wird das Projekt als karrierefördernd betrachtet. Präferenzen, Normen und Einstellungen sind oft sehr heterogen innerhalb der Gruppe.

Das kollektive Selbstbild kann auch wie ein Schutzschirm wirken und zu einer Absicherung führen. Um dieses „sichere" Selbstbild aufrechtzuerhalten, ist die Gruppe motiviert, ihre Außenwirkung konsistent zu halten. Wenn die Gruppe allerdings in eine Situation gerät, in der ihr Selbstbild infrage gestellt wird, wird sie versuchen, dieses zu erhalten. Sie investiert weiter in ihre Entscheidung, um vor sich selbst (als Gruppe) zu demonstrieren, dass die Entscheidung nicht nur richtig war, sondern darüber hinaus bewertet sie diese auch als gut. Sie bekräftigt damit nicht lediglich die einmalige Entscheidung (Expost-Rechtfertigung), sondern gibt sich der Illusion hin, weiterhin kompetent zu agieren. Darin liegt ihre Motivation, weiterhin in die Entscheidung, das Problem bzw. die Situation zu investieren. Die Gruppe bemüht sich damit 


\subsubsection{Eskalierendes Commitment}

um eine Überwindung des dissonanten Spannungszustandes ${ }^{33}$ und untermauert durch die Investitionen die Stärkung ihres kollektiven Selbstbildes.

Auch aus einem Intergruppenvergleich könnte sich das Phänomen speisen. Die soziale Gruppe vergleicht ihre Normen mit denen anderer Gruppen. Dabei werden die Intragruppendifferenzen minimiert und die Intergruppendifferenzen maximiert (vgl. Auer-Rizzi 1998, S. 68). Mit anderen Worten, indem die eigene Gruppe dem sozialen Vergleich standhält, wird die andere Gruppe abgewertet und die eigene Gruppe aufgewertet. Es entsteht also ein verstärktes Selbstwertgefühl der Gruppe. Sie fühlt sich

${ }^{33}$ Während des Entscheidungsprozesses und danach können Dissonanzen eine wesentliche Rolle spielen und die Entscheidung beeinflussen. Wenn zwei verschiedene Kognitionen aufeinandertreffen, kann Unwohlsein entstehen, was von Festinger (1957) als Dissonanz bezeichnet wird. Es ist das Streben des kognitiven Systems nach einem möglichst spannungsfreien Zustand (vgl. von Haeften 1999, S. 19). Für dissonanzauslösendes Verhalten gibt Festinger (1957) das anschauliche Beispiel eines Naturvolkes. Dieses vertrete die Auffassung, dass die menschliche Natur im Grunde gut sei. Diese Kognition „verträgt" sich aber nicht mit dem z.T. deutlich agressiven Verhalten der Heranwachsenden des Stammes. Die Dissonanz wird durch die Annahme beseitigt, dass sich böse Geister der Kinder bemächtigt hätten. Kognitionen („Menschen sind gut“) können aufgrund eines auftretenden Zustandes der Inkonsistenz von Kognitionen (,aggressive Jugendliche in Volksstamm“) umgedeutet werden (vgl. Beckmann 1984, S. 8). In diesem Fall soll die externe Kognition "böse Geister" die Dissonanz „vertreiben“. 


\subsubsection{Eskalierendes Commitment}

überlegen, machtvoll oder schlicht besser, weil das Vergleichsresultat für sie günstig ausgefallen ist. Diese kraftvolle Dynamik wurde in eindrucksvollen Experimenten, z.B. von Milgram (1974) oder Sherif (1953), dargestellt. Das kollektive Selbstbild der Gruppe führt zu einer falschen Selbstbestätigung, die unter rationalen Erwägungen zu keinen weiteren Investitionen geführt hätte. Statt die Notbremse zu ziehen und nicht weiter in die Entscheidung zu investieren, wird durch eine erneute Investition die Illusion geschaffen, das Richtige getan zu haben. Die erneute Investition ist die Rechtfertigung für die einstmals getroffene Entscheidung der Gruppe.

Becker-Beck und Wend (2008, S. 238 f.) haben eskalierendes Commitment in Gruppenentscheidungen vor dem Hintergrund der Theorie der sozialen Identität untersucht. In ihrem Experiment sollten Gruppen aus drei Personen über weitere Investitionen in ein riskantes Städtebauprojekt entscheiden. Die Investitionshöhe wurde als Indikator für eskalierendes Commitment angenommen und erwies sich als abhängig sowohl von der Stärke des Commitments als auch vom Ausmaß der Verantwortlichkeit. Kollektives Entscheidungsverhalten kann genauso „verbissen" sein und sich auch an einmal getroffene Entschei- 


\subsubsection{Eskalierendes Commitment}

dungen binden, obwohl ein hohes oder gar steigendes Risiko besteht.

Der Verpflichtung, sich kontinuierlich an einen Misserfolg zu halten (vgl. Auer-Rizzi 1998, S. 267), unterliegen Gruppen genauso wie individuelle Entscheider/-innen. Der Rechtfertigungs- und Zeitdruck, dem Organisationsmitglieder ausgesetzt sind, führt dazu, dass sie für ihre getätigten Investitionen "geradestehen“ müssen. Die Gruppe versucht, sich selbst und ihre Reputation nach außen (vor anderen Organisationsmitgliedern bzw. anderen kollektiven Instanzen) zu schützen, indem sie vorgibt, eine (einstmals) richtige Entscheidung getroffen zu haben und deshalb an dieser durch weitere Investitionen festhalten zu wollen. Das Risiko wird zugunsten eines positiven Gruppenbildes ignoriert oder heruntergespielt. Gründe, warum Gruppen dem eskalierenden Commitment ebenso anheimfallen wie Individuen, können u.a. Folgende sein:

- Reputation: Die Reputation (beim Individuum: das Selbstbild) der Entscheidergruppe ist bedroht. Das Widerrufen der Entscheidung würde einen Verlust der Gruppenreputation nach sich ziehen. 
- Selbstrechtfertigung: Die Gruppe muss die Entscheidung rechtfertigen, vor sich (Selbstrechtfertigung) und vor anderen (Reputation). Je mehr sie sich dafür verantwortlich fühlt, desto stärker sind das Commitment und das Interesse, an der Entscheidung festzuhalten und weiter zu investieren (Vgl. Ross/Staw 1987, S. 275.

- Dissonanz: Die Gruppenmitglieder versuchen, Spannungszustände (Dissonanz) zu verhindern, indem sie Eskalationstendenzen als dissonanzreduzierende Mechanismen anwenden. Die Eskalation wird verstärkt, wenn sich die Gruppe verantwortlich fühlt (vgl. Becker-Beck/Wend 2008, S. 238). Dissonanz tritt beim eskalierenden Commitment auf, wenn die individuellen Prozesse der Dissonanzreduktion eine einheitliche Richtung aufweisen (vgl. Schulz-Hardt 1997, S. 70).

- Framing: Je nachdem, wie eine Botschaft formuliert wird (bei gleichem Inhalt), wird sich das Verhalten des/der Empfängers/-in ändern, d.h., wenn die Gruppe z.B. die Wahl hat zwischen einem sicheren Verlust und der Möglichkeit eines größeren Verlustes, der aber das ursprünglich erwartete Er- 


\subsection{Zusammenfassung}

gebnis in Aussicht hat, wird die Entscheidung eher risikoaffin getroffen (Wahl des größeren Verlustes, d.h., es werden zusätzliche Ressourcen in die fehlerhafte Entscheidung investiert). Andersherum werden Entscheidungen risikoavers getroffen, wenn eine positive Entscheidungsaussicht formuliert wird, bei der aber das Risiko des Scheiterns höher ist (vgl. Staw 1997, S. 199 f.)

Eskalierendes Commitment ist ein häufiges Phänomen in Organisationen, und die englische Bezeichnung too much invested to quit syndrom beschreibt zugleich auch die Irrationalität dieses Defektes - eine Falle, in die getappt wird und die nicht nur Ressourcen bindet, sondern auch hohe Kosten verursachen kann. Auch Gruppen sind davor nicht gefeit.

\subsection{Zusammenfassung}

Entscheidungsverhalten wurde lange generell mit rationalem Entscheiden gleichgesetzt. Auch begrenzt rationales Verhalten steht meist in Verbindung mit fehlerhaftem Entscheiden. Der Fast-and-frugal-Ansatz von Gigeren- 


\subsection{Zusammenfassung}

zer, Todd und der ABC-Gruppe (1999) steht unter einem anderen Stern: Heuristisches Entscheidungsverhalten, das idealerweise ökologisch rational angewandt wird, ist eine evolutionsbiologische Entscheidungshilfe, die nicht nur angeboren ist, sondern auch noch gute Ergebnisse liefern soll. Die Fragestellung der Arbeit, ob dies auch in Gruppen der Fall sein könnte, wurde analysiert.

Heuristisches Entscheidungsverhalten von Gruppen ist geprägt durch die soziale Dynamik der Mitglieder. Die Entscheidungsregeln individueller Entscheidungen sind dabei die Basis für das Handeln der Gruppe. Hinzu kommen aber eigene Dynamiken, die sich aus der sozialen Dynamik der Gruppe ergeben. Es entstehen zahlreiche Defekte und Heuristiken, wenn eine Entscheidung in Gruppen getroffen wird. In dieser Arbeit wurden deshalb die sozialen Bewegungen exemplarisch angesprochen, um die Übertragbarkeit auf die heuristische Entscheidungsforschung in Gruppen (kollektive Ebene) darzustellen. Die Informationsverarbeitung und mit ihr der HiddenProfile-Effekt sind zentrale Aspekte, wenn es um die kognitiven Prozesse Einzelner und der Gruppe geht, da die heuristische Informationsverarbeitung nicht nur abgekürzt wird, sondern auch durch Gruppenprozesse eine 
Lenkung erfährt. Bereits bekanntes (geteiltes) Wissen wird in der Gruppe repetiert, und Neues (ungeteiltes Wissen) gelangt nicht in die Gruppensphäre. Die zustande gekommene Entscheidung der Gruppe (also der Output des Hidden Profile) kann deshalb heuristischer Natur sein. Auch Cognitive Maps wurden als soziales Phänomen aufgefasst und dem kollektiven Bewusstsein der Gruppe zugeordnet. Sie sind die inneren Modelle der Organisation. Grundlage der Idee ist, dass jede Gruppenentscheidung durch kognitive Repräsentationen geprägt wird. Cognitive Maps bilden die Repräsentation kollektiver Entscheidungsmuster und stellen dabei die Verfestigung der Bedeutungsschemata der Gruppenmitglieder dar. In Verbindung mit Heuristiken werden sie zu kognitiven Leitplanken bei der Entscheidung der Gruppe.

In Kapitel 3.4 konnte dargestellt werden, dass auch Gruppenentscheidungen genauso anfällig für Entscheidungsdefekte sind wie individuelle Entscheidungen. Soziale und individuelle Phänomene von Gruppenentscheidungen wurden deshalb anhand der Entscheidungsfehler Verantwortungsdiffusion, Kontrollillusion und eskalierendes Commitment exemplarisch dargestellt. Diese Gruppeneffekte beeinflussen das Entscheidungsverhalten von 


\subsection{Zusammenfassung}

Gruppen und können zu Fehlern im Entscheidungsprozess führen. 
4 Anwendungsbeispiel: Heuristische Entscheidungen bei der Personalauswahl

\section{Anwendungsbeispiel: Heuristische Entscheidungen bei der Personalauswahl}

Um eine spezifische Entscheidungssituation als Auswahlentscheidung näher zu betrachten, soll im Folgenden eine Gruppenentscheidung im Vordergrund stehen. Das Anwendungsbeispiel „Personalauswahl“ dient der Abgrenzung des heuristischen vom defizitären Entscheidungsverhalten und soll die Perspektive der Gruppe im Gegensatz zum Individuum analysieren.

Falsche Personalentscheidungen sind ein ausgeprägter Kostenfaktor in Organisationen. Dass eine unqualifizierte Personalauswahl einen wirtschaftlichen oder gar volkswirtschaftlichen Schaden bedeuten kann, wurde von vielen bereits hinreichend dargestellt (u.a. Kanning 2011, S. 72 f.; Naef 2001, S. 117). Die Konsequenzen der Personalauswahl haben oft langfristigen Charakter (Gehalts-, Abfindungs- und Wiederbesetzungskosten und Ressourcenbindung), sei es durch falsche Personaleinstellungen, die sich z.B. auf Schlüsselpositionen beziehen können, in denen die Organisation entscheidend mitgestaltet wer- 
4 Anwendungsbeispiel: Heuristische Entscheidungen bei der Personalauswahl

den kann (hierunter können Führungspositionen, aber auch Fachkräfte gefasst werden), oder durch unpassende interne Personalversetzungen mit strategischem Charakter für die Organisation und ihre Zielsetzungen. Die Bereitstellung von Personalkapazitäten lässt sich durch die Personalauswahlentscheidung realisieren. Es sollen die Geeignetsten für die vorgegebene Zielposition ausgewählt werden (vgl. Hell/Päßler 2011, S. 91). Die Auswahlentscheidung zugunsten externen oder internen Personals ist also eine Entscheidung mit weitreichenden Folgen für jede Organisation. Zwar steht und fällt die Existenz der Organisation selten mit der Entscheidung oder Versetzung einzelner Mitarbeiter/-innen, aber es kann durchaus zu einer Motivationsverschiebung oder einem Absenken des Leistungsniveaus bei einer Veränderung des Personals kommen.

Durch die Anwendung des Teilzeitbefristungsgesetzes sowie die Vereinbarung von Probezeiten ist es Organisationen möglich, sich von unpassenden Mitarbeitern/innen zu trennen, wenn sich nach Einstellung oder Versetzung herausstellt, dass die Vorstellungen der beiden Parteien nicht zusammenpassen. Damit es nicht zur 
4 Anwendungsbeispiel: Heuristische Entscheidungen bei der Personalauswahl

Trennung als Ultima Ratio kommt, muss die Auswahl des Personals in der Organisation einen hohen Stellenwert haben. Eine qualifizierte Personalauswahl kann als Erfolgsfaktor für Organisationen oder gar als Wettbewerbsvorteil angesehen werden, und die Fähigkeiten und Fertigkeiten von Fach- und Führungskräften sind Bausteine, die zu einer Wertschöpfung beitragen. Werden diese Trennungsmittel nicht ausgeschöpft, verbleiben die Mitarbeiter/-innen unter Umständen zu lange (Absentismus, Minderleistung) oder zu kurz in der Organisation (Fluktuation). Sie können erheblichen Schaden anrichten, wenn sie aufgrund von Fähigkeiten oder Motivation nicht auf die Stelle passen. Oder der/die Mitarbeiter/-in selbst ist mit einer unpassenden Stelle wenig glücklich, sodass sein/ihr Verhalten von Demotivation geprägt ist, vielleicht sogar bis hin zur Reaktanz. Die Konsequenzen können Absentismus, ineffizientes Arbeiten bis hin zur Sabotage sein.

Eine gute Personalauswahl, um geeignete Mitarbeiter/innen zu finden, stellt auf den Erfolg des eingestellten oder versetzten Personals ab. Um dies zu gewährleisten, gehen viele Organisationen dazu über, Personalent- 
4 Anwendungsbeispiel: Heuristische Entscheidungen bei der Personalauswahl

scheidungen von mehreren treffen zu lassen mit dem Ziel eines breiten Perspektiven- und Meinungsfeldes und damit einer vermeintlich höheren Auswahlkompetenz (vgl. Weuster 2012b, S. 62). Der Fokus der diagnostischen Personalforschung zielt jedoch weiterhin auf das Individuum als auswählendes Subjekt. Doch in der Organisation werden Personalentscheidungen in Gruppen getroffen. Mehrere Köpfe sollen eine gemeinsame Entscheidung treffen, um nicht einzelne Vorlieben in der Personaleinstellung und des Personaleinsatzes gegen das Organisationswohl wirken zu lassen; aber auch, weil man gemeinhin annimmt, dass bei mehreren Entscheidern/-innen die Qualität der Entscheidung eine bessere und objektivere ist.

Im folgenden Kapitel sollen Gruppenheuristiken (siehe Kapitel 3.3.2) in Verbindung mit dem kollektiven Anwendungsfall „Personalauswahl“ untersucht werden. Welche sozialen Prozesse haben Einfluss auf die heuristische Entscheidungsfindung der Gruppe? Welche Heuristiken können exemplarisch wirken? Und wie verhält sich die Gruppe im Gegensatz zum Individuum? Insbesondere im Hinblick auf die Personalauswahl sind intuitive Entschei- 
4.1 Personalauswahl als Gruppenentscheidung

dungsregeln ein Phänomen, das täglich in Organisationen Anwendung findet und deshalb auch so interessant ist. Die Gruppenmitglieder bewerten bei der Personalauswahl die Bewerberinformationen aus ihrer eigenen Perspektive, die anschließend abgeglichen und abgestimmt wird. Wie dabei heuristische Entscheidungsregeln im Entscheidungsprozess der Personalauswahl wirken können, soll im Folgenden dargestellt werden. Sie werden u.a. auch dahingehend untersucht, ob sie für die Personalauswahl eine Verzerrung des Entscheidungsprozesses oder eine Entscheidungshilfe sein können. Das Kapitel wird deshalb den Fokus auf die Wirkung von Gruppenheuristiken bei Personalentscheidungen legen.

\subsection{Personalauswahl als Gruppenentscheidung}

In der Personalauswahl tritt die Gruppe nicht zufällig zusammen. Ihr übergeordnetes Ziel ist klar definiert, und die Organisation gibt ihre Zielsetzung vor, dass eine Stelle in der Organisation zu besetzen ist. Damit die das Personalauswahlgremium keine natürlich formierte Gruppe, denn die Aufgaben werden geteilt, und jedem/jeder wird eine Rolle explizit (durch Stellenbeschreibung oder Arbeitsanweisung) oder implizit (durch soziale Phänomene 
4.1 Personalauswahl als Gruppenentscheidung

wie Rollenerwartungen) zugewiesen (vgl. Metz-Göckel 2002, S. 4). Es klingt zunächst einmal positiv, wenn alle wissen, was zu tun ist. Im Weiteren wird die Arbeit aber nicht bei Anforderungsprofilen, Personalzielsetzungen oder Rollenerwartungen von Personalverantwortlichen ansetzen, sondern sie wird heuristische Entscheidungswege und Entscheidungsregeln in der Personalentscheidung beleuchten. Denn was passiert „zwischen den Zeilen" einer standardisierten Personalauswahl? Ziele, Aufgaben und Vorgehensweise sind in der Realität selten so klar formuliert, dass daraus eine rationale Entscheidungsfindung resultieren kann. Aber was geschieht dann? Wie entscheidet eine Gruppe über Bewerber/innen, wenn sie doch keine oder wenige Leitplanken bzw. nur wenige Informationen hat?

Die Personalauswahlentscheidung ist eine zentrale Entscheidungssituation im Sinne der Organisation und eine komplexe Entscheidung im Sinne einer Problemlöseentscheidung (vgl. Kirsch 1970, S. 70). Problemlösung ist gleichbedeutend mit dem Versuch, eine Situation zu bewältigen, für die noch keine detaillierten Verhaltensweisen (z.B. aufgrund von Lernprozessen) zur Verfügung stehen (vgl. Höpfner 1997, S. 202). Auch wenn es orga- 
4.1 Personalauswahl als Gruppenentscheidung

nisationale Vorgaben zur Personalauswahl gibt, ist jede Auswahlsituation einzigartig, weil Bewerber/-innen, Gruppen, Stellenanforderungen etc. jedes Mal in einer anderen Konstellation mit anderen Merkmalen bewertet werden müssen. Deshalb kann es keine organisationalen Schemata geben, die so detailliert und standardisiert sind, dass sie für alle Auswahlsituationen taugen. Es kann deshalb auch von einer Entscheidung unter Unsicherheit gesprochen werden, weil Wahrscheinlichkeiten für Ereignisse, die aus der Wahl der Alternativen resultieren, nicht bekannt sind (vgl. Scholz et al. 2003, S. 11). Bei der personalwirtschaftlichen Auswahlentscheidung geht es um Ermittlung, Planung und Realisierung des Personalbedarfs zum Zwecke der Versorgung der Organisation mit Personal (vgl. Metz-Göckel 2002, S. 6).

Verschiedene Merkmale können die Entscheidungsart außerdem bestimmen: Entscheidungsträger/-innen, Entscheidungsanlass und Entscheidungsstruktur (vgl. Schüßler 1993, S. 42 f.). Für die Personalauswahlsituation bedeutet dies, dass der Entscheidungsträger die Gruppe ist, welche von der Entscheidungsstruktur innerhalb der Organisation beeinflusst wird. Der Entschei- 
4.1 Personalauswahl als Gruppenentscheidung

dungsanlass ist die zentrale Auswahlsituation am Ende des Personalbeurteilungsprozesses.

Das Kollektiv in der Entscheidungssituation der Personalauswahl hat folgende Ausprägungen:

\begin{tabular}{|l|l}
\hline Aspekt & Ausprägung \\
\hline Entscheidungstrāger & $\begin{array}{l}\text { Gruppe aus 2-5 Personen (in mehrstufigen Prozessen } \\
\text { kōnnen eine Vielzahl von weiteren Personen beteiligt } \\
\text { sein) }\end{array}$ \\
\hline Art des Kollektivs & Formelles Kollektiv mit Zielsetzung \\
\hline Entscheidungsanlass & $\begin{array}{l}\text { Personalauswahim Sinne des organisationalen } \\
\text { Anforderungsprofils (Zielsetzung) }\end{array}$ \\
\hline
\end{tabular}

Abbildung 10: Einordnung Personalauswahlsituation, in Anlehnung an Grünig und Kühn (2012, S. 225).

In der Personalauswahl der Organisation ist der Entscheidungsträger für die Personalentscheidung also meist eine Gruppe, die mit der Aufgabe betraut wird, über die Neueinstellung oder Versetzung gemeinsam zu entscheiden. Die Gruppenmitglieder können wechseln, weil die Personalauswahlprozesse häufig mehrstufig sind (Assessment-Center, Vorstellungsgespräch, Probearbeiten etc.) (vgl. Weuster 2012a, S. 29). Mit einer Gruppe als Entscheidungsträger soll die Problemlösefähigkeit der Organisation erweitert werden. Damit delegiert die Orga- 
4.1 Personalauswahl als Gruppenentscheidung

nisation die Entscheidungen an ein aufgabenspezialisiertes Gremium. Das Urteilsvermögen der Personalentscheidung soll dadurch eine positive Aufladung erfahren. Im Gegensatz zum Zweiergespräch (ein/eine Interviewer/-in, ein/eine Bewerber/-in) soll der Einsatz von mehreren von Vorteil sein, da so auch die Beeinflussung der Auswahl durch unterschiedliche Kombinationen von Entscheiderpersönlichkeit und Bewerberpersönlichkeit relativiert werden kann (vgl. Weuster 2012b, S. 56).

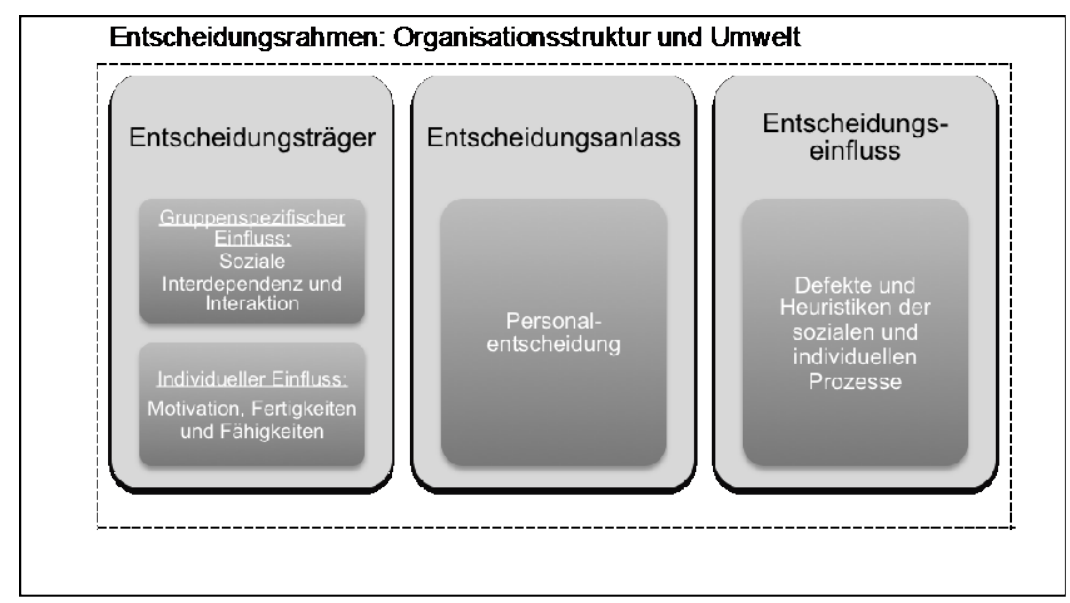

Abbildung 11: Einflüsse auf die Entscheidungssituation „Personalauswahl" der Gruppe (eigene Darstellung). 
4.1 Personalauswahl als Gruppenentscheidung

Der Entscheidungsträger "Gruppe“ und der Entscheidungsanlass „Personalauswahl“ geben das Gerüst für die weitere Analyse vor (siehe Abbildung 11). Sie werden umrahmt von der Organisationsstruktur (siehe Kapitel 2.1) und ihrer Umwelt. Beispiele für konkrete organisationsstrukturelle Rahmenbedingungen für die „Personalentscheidung" sind u.a.: Strukturiertheit von Entscheidungsprozessen, Rechenschaftsverpflichtung sowie Feedbacksysteme (vgl. Koppers 2013, S. 28). Dabei können durch das organisationale Umfeld und seine Mitglieder Wechselwirkungen entstehen. Personalentscheidungen hängen u.a. von den Organisationsteilnehmenden, den Organisationsprozessen und den Charakteristika der Organisation ab (vgl. Cascio/Reynolds 2011, S. 65). Zu der Organisationsumwelt gehören z.B. die wirtschaftlichen Gegebenheiten, unter denen die Gruppe agiert. Zum Beispiel stellt sich eine Auswahlentscheidung in Zeiten des Arbeitskräftemangels anders dar als eine Personalauswahl mit großer Auswahl an Bewerbern/innen.

Die Gruppenentscheidung setzt sich aus individual- und gruppenspezifischen Komponenten zusammen: 


\section{Individualspezifische Komponenten:}

Entscheidungen sind u.a. von den individuellen Fähigkeiten und Fertigkeiten der einzelnen Gruppenmitglieder geprägt sowie von deren Einzelmotivation (vgl. Cascio/Reynolds 2011, S. 65). Die Fähigkeiten Einzelner haben einen ganz entscheidenden Einfluss auf die Gruppenentscheidung, z.B. durch ihre Qualifikationen. Gut geschulte Beobachter sollen im Bewerbungsinterview eher eine objektive Bewertung abgeben können als Ungeschulte. Hinzu kommt eine motivatorische Komponente: Je mehr sich der/die einzelne Beobachter/-in mit dem Auswahlprozess beschäftigt, desto mehr beschäftigt er/sie sich z.B. auch mit den Bewerberinformationen. Je nachdem, wie diese Mischung ausgestaltet ist, gestaltet sich auch die Gruppenleistung. Daten und Wahrnehmungen der Gruppenmitglieder müssen in ein Gesamturteil der Gruppe integriert werden (vgl. Kanning 2004, S. 268).

\section{Gruppenspezifische Komponenten:}

$\mathrm{Zu}$ den gruppenspezifischen Komponenten gehören die soziale Interdependenz (das Bezugsverhalten und das Bewusstsein, dass das Ergebnis von anderen Gruppen- 
4.1 Personalauswahl als Gruppenentscheidung

mitgliedern abhängt) und die soziale Interaktion (hierzu gehören Konflikte sowie weitere Interaktionskomponenten, wie Führung, Kommunikation und weitere intragruppenbezogene Prozessdynamiken, wie Gruppendruck etc.) (vgl. Drewes et al. 2011, S. 223).

Die Steuerungsvariablen der Organisation beeinflussen den Entscheidungsprozess sowohl des Individuums als auch der Gruppe. Die Organisation handelt dann als einheitlicher kollektiver Akteur, wenn sie das Handeln der Teilnehmenden beim Personalauswahlprozess beeinflusst. Die Strukturiertheit kollektiven Verhaltens als besonderes Merkmal des kollektiven Handelns prägt Personen. Die Organisationsstruktur kanalisiert, unterstützt oder behindert das Verhalten der Akteure (vgl. Martin 1995, S. 8). Aus dieser Perspektive handelt die Gruppe bei der Personalentscheidung als autorisiertes Gremium des institutionalen Handelns, aus der sich die gruppenspezifische Komponente der Entscheidung ergibt.

Im Folgenden geht es um den Einfluss der Organisation auf die Personalauswahl. In Kapitel 2.1 wurde dieser Einfluss anhand von Organisationsmerkmalen wie der Organisationsstruktur beschrieben. Der organisationale Ein- 
4.2 Einfluss der Organisation bei der Personalauswahl

fluss soll nun in den Anwendungsfall der Personalaus-

wahl Eingang finden. Konkret soll zunächst kurz die Unterscheidung zwischen formellen und informellen Einflüssen auf den Einstellungsprozess dargestellt werden, d.h., wie die Organisation in die Personalauswahl "eingreift", sodass Gruppenmitglieder in ihrer Entscheidung beeinflusst werden.

\subsection{Einfluss der Organisation bei der Personalauswahl}

Einen Einfluss auf den Entscheidungsprozess kann die Organisation durch die organisationale Vorgabe von Steuerungsvariablen vor dem Hintergrund ihres eigenen Zielstrebens ausüben. Die Organisation trifft aber meist nur mittelbar Entscheidungen selbst, z.B., indem sie organisationale Vorgaben macht. Diese können allenfalls bei den Beteiligten zu einem gemeinsamen Verständnis oder zu kooperativen Zielen bei der Personalauswahl führen. Die Grundlage dafür ist u.a. die Organisationsstruktur (siehe Kapitel 2.1). 
4.2 Einfluss der Organisation bei der Personalauswahl

Der organisationale Einfluss kann sich aus zwei Parametern speisen:

\section{Formelle Prozesse}

Die Organisation handelt als Einheit oder einheitlicher Akteur, wenn sie Handlungsvorgaben macht. Standardisierung ist dabei eine Art der Organisationsfestlegung von Personaldiagnoseprozessen (siehe Merkmale der Organisationsstruktur in Kapitel 2.1), z.B. durch Arbeitsanweisungen, Anforderungsprofile in Stellenausschreibungen, Prozessvorgaben (der Einsatz von Headhuntern/-innen bei der Auswahl von Führungskräften), Einhaltung gesetzlicher Vorgaben (wie die des AGG), Betriebsratsbeteiligung, Code of Conduct oder Werteverpflichtungen im Rahmen der Personalpolitik des Unternehmen etc.

\section{Informelle Prozesse}

Anderseits werden Organisationseinflüsse auch durch den „Geist der Organisation“ geprägt. Die Organisationsmitglieder haben gewisse Muster „im Kopf“, die die Gruppenmitglieder durch (langjährige) Organisationszugehörigkeit aus der Organisation „filtern“. Es ist das kollektive Bewusstsein der Organisation, das die Entschei- 
4.2 Einfluss der Organisation bei der Personalauswahl

dungsweise der Gruppe prägt: „The basic idea is that knowledge in very large networks of very simple processing units resides in patterns of connection, not in individuated local symbols“ (Roberts/Weick 1993, S. 359). Der Einfluss muss nicht fixiert sein, sondern kann auch informeller Natur sein (z.B. gelebte Unternehmenskultur oder nicht standardisierte Personalnormen etc.). Auch eine informationelle oder nicht institutionalisierte Einstellungspolitik mag sich daraus ergeben, z.B., wenn die Einstellungsrichtlinien „schwarz auf weiß" vorgegeben sind, aber das Einstellungsgremium anderen, informellen Entscheidungskriterien folgt.

In welcher kognitiven Weise sich der Einfluss der Organisationen in der Gruppe manifestiert, wurde in Kapitel 3.3.3.3 durch die räumlichen Landkarten (Cognitive Maps) exemplarisch dargestellt. Die Gruppe trifft die Auswahl der Personalie zwar nach eigenen Regeln, aber der Grundtenor kann von der Organisation selbst kommen. Dies geschieht also nicht nur durch formelle Prozesse, die die Organisation vorgibt, sondern auch durch informelle Prozesse, die ihren Ursprung in den Cognitive Maps haben können. Zur Vorbereitung der Entscheidung 
4.2 Einfluss der Organisation bei der Personalauswahl

wenden die einzelnen Organisationsmitglieder ihre eigenen Entscheidungsregeln an (jeder/jede macht zunächst eigene Vorannahmen). Auch durch diese individuellen Entscheidungsregeln fließt schon das „Blut“ der gesamten Organisation.

Beispiel: Der/die neue Abteilungsleiter/-in möchte für seine/ihre Vertriebsabteilung einen redegewandten und durchsetzungsstarken Einzelkämpfer als Außendienstmitarbeiter/-in einstellen. Es ist aber organisationsüblich, dass die Außendienstmitarbeiter/-innen Personen sind, die unterstützend zusammenarbeiten und nicht als Einzelkämpfer Produkte verkaufen. Dies kann zu einem Dissens in der Einstellungspolitik der Organisation führen. Durch divergierende Kognitionen können Interessenskonflikte im Entscheidungsprozess ausgelöst werden (vgl. Ahrens 1976, S. 29). Denn genauso wie Individuen können sich Gruppen vor Entscheidungen in Konflikten befinden, und diese können sowohl eigene Konflikte als auch Intragruppenkonflikte (soziale Konflikte) darstellen. Das Beispiel zeigt einen Interessenkonflikt des Individuums (Abteilungsleiter/-in) mit der Organisation und greift auch den Einfluss der Organisation auf den Einstellungs- 
4.3 Qualität und Rahmen der Personalentscheidung

prozess auf. Einflüsse jedweder Art bestimmen auch die Qualität und den Rahmen der Personalentscheidung.

\subsection{Qualität \\ und \\ Personalentscheidung}

Rahmen

der

Auch unter Zuhilfenahme von diagnostischen Auswahlverfahren, trotz der Kontrolle zahlreicher Variablen oder der Anwendung von Algorithmen ist eine rational intendierte Personalentscheidung in einer sozialen Umwelt schwer denkbar. Entscheidungen werden deshalb oft heuristisch getroffen. Sie sind in der Personalauswahl schon deshalb nicht mehr auszuschließen, weil Personalverantwortliche in Organisationen einem steigendem Entscheidungs- und Zeitdruck ausgesetzt sind. Diesem Druck kann u.U. nur durch abgekürzte Entscheidungen, eine geringere Informationsgenauigkeit und eine reduzierte Entscheidungsschnelligkeit begegnet werden, weshalb sich zwingend die Frage stellt, ob Heuristiken eher hinderlich für die Personalauswahl sind oder ob sie ihr sogar zum Vorteil gereichen können. Allgemein wurde diese Frage bereits in Kapitel 3.3.1 behandelt und soll nun konkretisiert werden. 
4.3 Qualität und Rahmen der Personalentscheidung

Die Qualität von Personalentscheidungen zu bestimmen, ist so komplex wie das Treffen der Entscheidung selbst, da unterschiedliche Interessengruppen (Bewerber/-innen, Personaler/-innen, Führungskräfte, Gewerkschaften etc.) unterschiedliche Erfolgskriterien angeben (vgl. Cascio/Reynolds 2011, S. 60). Auch Entscheidungsprozesse verlaufen sehr unterschiedlich. Sie sind häufig nicht strukturiert oder nur teilstrukturiert, und oft gibt es in der Organisation kein bestimmtes Konzept, wie die personelle Auswahlentscheidung zu treffen ist. In der Auswahlsituation sollen dann Entscheidungen über Bewerber/-innen für Berufe, Stellen oder Tätigkeiten getroffen werden. Dies können z.B. mehrere Stellen sein, auf die es nur einen/eine Bewerber/ -in gibt, oder eine Stelle, für die es mehrere Bewerber/innen gibt, sowie zahlreiche Varianten davon (vgl. Nerdinger/Rosenstiel 2011 S. 176). Die Wahl der diversen Diagnosemethoden für die Auswahl richtet sich nach dem Verlauf, den Beteiligten (z.B. eignungsdiagnostische Kompetenz) und den (situativen) Rahmenbedingungen (vgl. Koppers 2013, S. 13). Damit ist der Bewertungsrahmen der Personalentscheidung vielfältig, komplex und 
4.3 Qualität und Rahmen der Personalentscheidung

schwer abgrenzbar, wenn es um die Betrachtung von sozialen Variablen zur Bestimmung der Entscheidungsqualität der Gruppe geht.

Das Ziel einer geeigneten Personalauswahl besteht darin, Mitarbeiter/-innen als künftige Organisationsmitglieder zu finden, deren beruflicher Erfolg durch die diagnostische Auswahl der Bewerber/-innen vorhergesagt werden soll (vgl. Hell/Päßler 2011, S. 91). Diese Vorhersage gestaltet sich bisweilen schwierig. Verschiedene Kriterien zur Messung der künftigen Leistung spielen eine Rolle. Für die Personalauswahl stellen sich verschiedene Herausforderungen:

1. Verfahrensauswahl und Definition der Auswahlkriterien (welches Diagnoseverfahren soll welches Ergebnis erzielen?)

2. Gewichtung (welche Anforderungen sind für die Zielposition wichtig?)

3. Sichtung der Informationen (wie kann sichergestellt werden, dass alle Informationen vorliegen, die für die Entscheidung relevant sind, und alle 
4.3 Qualität und Rahmen der Personalentscheidung

Entscheider/

-innen gleich gut informiert sind?)

Erforderlich für die qualitative Personaldiagnostik sind geeignete Messinstrumente, die es ermöglichen, alle drei Ansprüche zu vereinen. Dennoch ist die Auswahl dieser Messinstrumente eine Herausforderung für die Organisationen, der sie sich oft dezidiert stellt. Eine Organisation sieht sich mit zahlreichen Hindernissen bei der Personalauswahl konfrontiert. Sie muss sich den oben genannten Herausforderungen stellen und kann dabei an ihre Grenzen stoßen. Diese Grenzen im organisationalen Entscheidungsverhalten bei einer Personalentscheidung wirken auf das Entscheidungsverhalten der Gruppe und zeichnen sich durch Folgenden Merkmale aus (vgl. Sanders/Kianty 2006, S. 185):

- Informationsgrenzen: Informationen und Wissen sind oft nur begrenzt verfügbar, und nicht jedem Gruppenmitglied liegen die gleichen Informationen vor. Informationen werden bei der Personalauswahl auch über die Wahrnehmung des/der Bewerbers/-in produziert, wenn sie weitergegeben werden. 
- Die Grenzen der Antizipation: Die Konsequenzen einer Entscheidung abzusehen, ist oftmals schwer für die Organisationsteilnehmenden. Zum Beispiel ist es häufig schwer einzuschätzen, wie ein/eine Bewerber/-in sich im Organisationsgeschehen nach der Einstellung verhält. Die Entscheidergruppe hat die Aufgabe, anhand von Informationen über die Stelle ${ }^{34}$ und die Bewerber/-innen eine Prognose über deren künftigen beruflichen Erfolg zu geben.

- Begrenzung der Alternativenauswahl: Auch bei der Auswahl der Entscheidungsmöglichkeiten eröffnet sich nicht das gesamte Spektrum der machbar möglichen Auswahl im Entscheidungsprozess. Limitiert wird die Alternativenauswahl z.B. durch (technologische) Fortschritte, die zu einem gewissen Zeitpunkt noch nicht bekannt oder erforscht waren. Wenn also den Gruppenmitglieder nicht alle relevanten Alternativen vorliegen, fällt die Entscheidung aufgrund eines ggf. begrenzten Informationshorizontes. Mit einem al-

\footnotetext{
34 Anforderungsprofile entlasten die Anwendenden in ihrer Informationsverarbeitung und Einschätzung stellenrelevanter Informationen. Sie sind die Basis für die Auswahlentscheidung, dient dem besseren Vergleich und stellt vor allem eine Entscheidungsregel dar, um eine begründete und nachvollziehbare Entscheidung zu treffen (vgl. Koppers 2013, S. 17).
} 
4.3 Qualität und Rahmen der Personalentscheidung

ternativen Horizont wäre die Entscheidung ggf. anders ausgefallen. Beispiel: Die Einführung eines neues Produktes war absehbar, wozu ein spezielles Bewerber/-innen Know-how langfristig vonnöten war, aber diese Information ist nicht zur Entscheidergruppe gelangt.

Neben den Grenzen des Entscheidungsverhaltens für die Gruppe werden Personalentscheidungen sowohl in Abhängigkeit vom sozialen Kontext und der Organisation als auch in Abhängigkeit von den Individuen getroffen (vgl. Cascio/Reynolds 2011, S. 65). Das heißt, Ränkespiele, um den eigenen Einfluss zu stärken, Konflikte, die in die Personalauswahl hineingetragen werden, dort aber nichts zu suchen haben, sind auch Komponenten der Personalentscheidung. Menschliches Entscheidungsverhalten wird grundsätzlich von sozialen Phänomenen begleitet. Eine kollektive Entscheidung bei der Personalauswahl ist deshalb herausfordernd, weil die beteiligten Personen z.B. stark divergierende Zielsysteme mitbringen. Da bei einer formellen Gruppe die Zielsysteme Einzelner nicht automatisch qua Zusammenarbeit zu kollektiven Zielen aggregiert werden, gestaltet sich häufig 
4.3 Qualität und Rahmen der Personalentscheidung

eine gemeinsame Entscheidungsfindung schwierig (vgl. Grünig/Kühn 2012, S. 256). Gruppenentscheidungen zeichnen sich zwar durch Meinungsvielfalt aus, jedoch stehen häufig diese sozialen und individuellen Phänomene einer zufriedenstellenden Entscheidungsfindung entgegen. Die Meinungsvielfalt kommt selten voll zum Tragen weil die Organisationsteilnehmenden, die in Entscheidungsgruppen organisiert sind, einen limitierenden Einfluss auf den Gruppenprozess haben (vgl. Neumer 2009, S. 12).

Die Grenzen und Anforderungen sind der Rahmen einer organisationalen Personalentscheidung und bestimmen auch ihre Qualität. Die Qualität der Personalentscheidung lässt sich aber nur schwer bemessen, weil die Ansprüche jeder Organisation andere sind. Diese herauszufiltern, gelingt nur selten, denn es stellt sich die Frage, was eigentlich die Normen der Organisation für eine angemessene Personalauswahl sind und was eine gute Personaleinstellung und der damit einhergehende Prozess bedeuten. Es stellt eine Herausforderung dar, Umweltphänomene von Gruppen zu bestimmen und die Umweltdynamik als Einfluss zu messen und anschlie- 
4.3 Qualität und Rahmen der Personalentscheidung

ßend eine Aussage über die Qualität zu treffen. So verhält es sich auch mit der Personalauswahl. Abstrakte Kriterien für eine angemessene Personalauswahl können von Organisationsteilnehmenden schnell gefunden werden, und bei Adjektiven, wie zuverlässig, fleißig, mitdenkend etc., werden viele zustimmen. Aber konkrete Kriterien für die einzelne Stellenauswahl zu beschreiben, gestaltet sich oft schwierig, da die Meinungen stark heterogen sind.

Häufig hängt eine Bewertung der Entscheidungsqualität auch mit den Umständen zusammen, in denen eine Entscheidung getroffen werden soll, und unter welchen Bedingungen diese stattfindet (z.B. saisonale Einflüsse, betrieblicher Druck von Vorgesetzten, Zeitnot etc.). Bei additiven Aufgaben wie Akkordarbeit fällt eine Messung der Entscheidungsqualität noch leicht: Ob sich der/die Einzelne anstrengt oder nicht, lässt sich am Gruppenergebnis (z.B. Höhe der Stückzahl) ablesen, wenn alle anderen Variablen gleich bleiben. Auch am Beispiel Tauziehen kann beobachtet werden, dass sich das einzelne Gruppenmitglied anstrengt. Aber wenn z.B. Vergleiche zu anderen Gruppenmitgliedern gezogen und Motivationseffekte (wie Trittbrettfahren) etc. einbezogen werden, wird 
4.3 Qualität und Rahmen der Personalentscheidung die Bewertung der Entscheidungen schon komplexer (vgl. Gigerenzer/Gaissmaier 2006, S. 15). Im Gegensatz zu den individualspezifischen Entscheidungskomponenten kann der Einfluss von gruppentypischen Elementen nur indirekt bestimmt werden. Es besteht keine Übereinstimmung in der sozialpsychologischen Forschertradition hinsichtlich der Frage, inwieweit die Interaktion zwischen den Gruppenmitgliedern die Entscheidungsleistung beeinflusst (vgl. Reimer 1996, S. 18).

Die Interaktion kann sich bei der Personalauswahl aus der Wahrnehmung und Diagnose der Beteiligten speisen und ist der Schlüssel für die Einigung in der Gruppe. Eine Einigung der Gruppe ist für die Personalauswahl entscheidend, weil die Auswahl meist auf eine einzustellende Person fallen muss. Im Gegensatz zu anderen Arten der Entscheidung ist kein Abweichen möglich, oder anders ausgedrückt: Es wird nur ein/eine Kandidat/-in eingestellt und keine Kompromissentscheidung getroffen, indem jeweils ein bisschen von den verschiedenen Alternativen in die Endauswahl einfließen kann. Wenn Gruppenentscheidungen in Laborexperimenten untersucht werden, richtet sich die Bewertung von Personalent- 
4.3 Qualität und Rahmen der Personalentscheidung

scheidungen häufig nach der Übereinstimmung der Beurteilenden (vgl. Koppers 2013, S. 14).

Die Qualität der Entscheidung richtet sich bei der Personalauswahl nach dem Ergebnis, das die Gruppe trifft, denn sie ist das Ergebnis der abschließenden Entscheidung der Gruppe und damit ihre Gruppenleistung. Jedoch liegt in einem naturalistischen Kontext (im Gegensatz zu den Laborexperimenten) die Schwierigkeit darin, dass sich die Entscheidungsqualität erst nach der Einstellung der Bewerber/-innen zeigt. Diese kann sich erst im Laufe der Betriebszugehörigkeit des/der eingestellten Kandidaten/-in als "gut" und im Sinne der Organisation als angemessen erweisen. Oft zeigt sich die Leistung, die der/die Mitarbeiter/-in erbringt, und wie er/sie sich in seine/ihre Arbeitsumgebung einpasst, erst weit nach der eigentlichen Personalentscheidung. Hinzu kommt, dass diese Information die Gruppe oft nicht erreicht. Selten erfährt die Gruppe, welche Entscheidung "richtig“ war und welche nicht. Dass heißt, ob ihre Personalentscheidung ursächlich für den Einstellungserfolg war, erfahren sie oft nicht. Zudem können die Teilnehmenden im Nachhinein häufig nicht mehr rekonstruieren, welche 
Entscheidungsregeln sie "damals" angewandt haben und ob eine Personalentscheidung gut, brauchbar, angemessen oder katastrophal gewesen ist. Nicht nur die Entscheidung selbst, auch ihre Bewertung liegt oft im Auge des Betrachters, d.h. wird mittels subjektiver Normen beurteilt. Zum Beispiel kann sich eine "gute" Personalauswahl auch in alternativen Bewertungen zeigen, indem der/die neue Mitarbeiter/-in andere Organisationsmitglieder motiviert, von seinen/ihren Fertigkeiten und Fähigkeiten zu lernen, oder indem er/sie besonders gut Organisationsprozesse koordinieren kann.

Einige Messinstrumente werden im Folgenden skizziert, um einen Überblick zu erhalten und den Anwendungsfall der Personalauswahl greifbarer zu machen. Denn bevor es zu einer Personalentscheidung kommt, laufen zahlreiche Prozessschritte ab, die als eine Reihe von diagnostischen Vorabentscheidungen gesehen werden können. 
4.4 Personaldiagnostische Prozesse und ihre Hindernisse

\subsection{Personaldiagnostische Prozesse und ihre Hindernisse}

Die Personalauswahlentscheidung ist das Endergebnis der Personaldiagnostik. Generell bezieht sich die Eignungsdiagnostik auf verschiedene Fachgebiete der Psychologie, u.a. die Psychotherapie oder die klinische Psychologie. Die Eignungsdiagnostik steht dabei im Dienste der Personalauswahl, auch wenn sie in der Beratung häufig auf den klinischen Anwendungsfall bezogen ist (vgl. Hell/Päßler 2011, S. 19). Ihre Instrumente bestehen aus Erhebung (z.B. durch Befragung der Fachvorgesetzten zu den Anforderungen der Stelle), Informationsverarbeitung und Verwendung. Es kann unterschieden werden zwischen diagnostischen Verfahren (Tests, Analysegespräche oder Assessment-Center) ${ }^{35}$ oder Kompetenzen (im Sinne von Qualifikationen und Prüfungen) (vgl. Kersting 2008, S. 19). Diagnostik ist ein Instrument zur

${ }^{35}$ Für Managementpositionen oder besondere Fachaufgaben werden z.B. Kenntnisse und Fähigkeiten im Rahmen eines AssessmentCenters oder anhand von Arbeitsproben erfasst, in denen der/die Bewerber/-in sein Wissen durch Ausführung von arbeitsplatzverwandten Aufgaben unter Beweis stellen kann (vgl. Cascio/Reynold 2011, S. 60). 
4.4 Personaldiagnostische Prozesse und ihre Hindernisse

Optimierung der Qualität von Entscheidungen, denn Eignungsdiagnostik ist die psychologische Systematik von Entscheidungen zur Erhaltung von Qualitätsstandards (vgl. Kersting 2008, S. 11). Es soll damit das Verhalten von Menschen in Bezug auf ihren beruflichen Erfolg vorhergesagt werden. Dabei können Fragebögen zur Personalauswahldiagnostik hohe Vorhersagewerte für den beruflichen Erfolg ausweisen. Zum Beispiel sind die kognitive Leistungsfähigkeit (Intelligenz) sowie Persönlichkeitsunterschiede Merkmale, um die berufliche Leistung valide abzubilden (vgl. Bickle 2011, S. 114). Intelligenzund Persönlichkeitstests wurden als valide Werkzeuge zur Vorhersage der beruflichen Leistung getestet (siehe Tabelle 4).

Welche Prozessverfahren zur Personalauswahl angewandt werden, variiert von Organisation zu Organisation. Grundsätzlich wird jede organisatorische Entscheidung durch unterschiedliche Aspekte beeinflusst, und die personaldiagnostische Auswahl bildet hier keine Ausnahme. Zum Beispiel kann der Auswahlprozess zur Auswahl von Führungskräften stets ein Assessment-Center in Verbindung mit Vorstellungsgesprächen und gemeinsamen 
4.4 Personaldiagnostische Prozesse und ihre Hindernisse

Abendessen vorsehen und standardisiert vorgegeben sein. An diese Auswahlregel als eine von der Organisation vorgegebene Diagnosemethode muss die Gruppe sich dann halten.

Neben dem Testinstrumentarium (z.B. Fragebögen) gibt es zahlreiche andere Verfahren der Einstellungsdiagnose (siehe Tabelle 4). Die Informationen über Bewerber/innen werden sowohl direkt (in Tests, Interviews etc.) als auch indirekt (z.B. durch Referenzen von früheren Arbeitgebern) erhoben. Einstellungsinterviews bilden hierbei den personaldiagnostischen Kern der Personalentscheidung, denn ohne sie werden gemeinhin keine Einstellungsentscheidungen getroffen. Allerdings darf kein qualitativer Rückschluss dahingehend getroffen werden, dass die Anwendungshäufigkeit der Verfahren ein Garant für eine hohe Validität ist (vgl. Cascio/Reynolds 2011, S. 66). Gleichfalls ist eine hohe Validität auch keine Anwendungsverpflichtung für die praktische Umsetzung, weil die Verfahren unter Umständen zu teuer sind und einen hohen Einsatz und Bedarf an Kompetenzen erfordern. In zahlreichen Organisationen werden Personalauswahlverfahren nur deshalb eingesetzt, weil sie möglicherweise 
4.4 Personaldiagnostische Prozesse und ihre Hindernisse

$\overline{\text { praktikabel sind oder/und sozial akzeptiert werden, nicht }}$ aber, weil sie valide sind (vgl. Kersting 2008, S. 41). Nachfolgende Tabelle 4 zeigt die Vielfalt der Auswahlverfahren und ihre Anwendungshäufigkeit. Schuler (2002, S. 12 f.) befragte die 550 umsatzstärksten deutschen Unternehmen nach ihrer Personalauswahl und verglich die Antworten mit den vorherigen Ergebnissen (aus dem Jahr 1993). Das am zweithäufigsten angewandte Verfahren ist das strukturierte Einstellungsinterview. Auch dieses erweist sich nach Schuler (2002) nicht als vollendet valide. Schuler maß eine Validität von .44. Niedriger bewerten Fific und Gigerenzer (2014, S. 3) die Validität und geben sie sogar nur mit .10 bis .22 an. Die Fähigkeit von Interviews, den künftigen Erfolg valide vorherzusagen, wird in der Organisationspsychologie oft als bescheiden bewertet, sie reiche vielfach nicht über die Zufallserwartung hinaus (vgl. Nerdinger/Rosenstiel 2011, S. 159). 
4.4 Personaldiagnostische Prozesse und ihre Hindernisse

Tabelle 4: Validität und Anwendungshäufigkeit von Personalauswahlverfahren in Deutschland (Schuler et al. 1993, Schuler 2007), sortiert nach Einsatzhäufigkeit der Auswahlverfahren im Jahr 2003.

\begin{tabular}{|c|c|c|c|}
\hline & $\begin{array}{l}\text { Einsatzhäu- } \\
\text { figkeit der } \\
\text { Auswahlver- } \\
\text { fahren } 2003 \\
\text { (Schuler } \\
\text { 2007) }\end{array}$ & $\begin{array}{l}\text { Einsatzhäu- } \\
\text { figkeit der } \\
\text { Auswahlver- } \\
\text { fahren } 1993 \\
\text { (Schuler et } \\
\text { al. 1993) }\end{array}$ & $\begin{array}{l}\text { Diffe- } \\
\text { renz } \\
2003- \\
1993\end{array}$ \\
\hline Analyse der Bewerbungsunterlagen & $99 \%$ & $98 \%$ & $1 \%$ \\
\hline $\begin{array}{l}\text { Strukturiertes Einstellungsinterview durch } \\
\text { Personalabteilung }\end{array}$ & $82 \%$ & $70 \%$ & $12 \%$ \\
\hline Analyse von Online-Bewerbungsunterlagen & $71 \%$ & & \\
\hline Personalfragebogen & $69 \%$ & & \\
\hline $\begin{array}{l}\text { Strukturiertes Einstellungsinterview durch } \\
\text { Fachabteilung }\end{array}$ & $64 \%$ & $49 \%$ & $15 \%$ \\
\hline Assessment-Center & $58 \%$ & $39 \%$ & $19 \%$ \\
\hline Zusätzlich eingeholte Referenzen & $57 \%$ & $71 \%$ & $-14 \%$ \\
\hline $\begin{array}{l}\text { Unstrukturiertes Einstellungsinterview Fach- } \\
\text { abt. }\end{array}$ & $51 \%$ & $69 \%$ & $-18 \%$ \\
\hline Arbeitsproben & $45 \%$ & $44 \%$ & $1 \%$ \\
\hline Gruppengespräch/Diskussion & $42 \%$ & $51 \%$ & $-9 \%$ \\
\hline Leistungstest, z.B. Bürotest & $41 \%$ & $47 \%$ & $-6 \%$ \\
\hline Medizinische Begutachtung & $41 \%$ & $64 \%$ & $-23 \%$ \\
\hline $\begin{array}{l}\text { Unstrukturiertes Einstellungsinterview Per- } \\
\text { sonalabt. }\end{array}$ & $34 \%$ & $57 \%$ & $-23 \%$ \\
\hline Strukturiertes telefonisches Interview & $32 \%$ & & \\
\hline Intelligenztest & $30 \%$ & $34 \%$ & $-4 \%$ \\
\hline Standardisierter Online-Personalfragebogen & $22 \%$ & & \\
\hline Persönlichkeitstest & $20 \%$ & $21 \%$ & $-1 \%$ \\
\hline Grafologisches Gutachten & $2 \%$ & $9 \%$ & $-7 \%$ \\
\hline Biografischer Fragebogen & $2 \%$ & $21 \%$ & $-19 \%$ \\
\hline Stichprobengröße N & 125 & 105 & \\
\hline
\end{tabular}


4.4 Personaldiagnostische Prozesse und ihre Hindernisse

Obwohl es zahlreiche wissenschaftliche Beiträge in Zeitschriften und anderen berufsständischen Medien gibt, in denen die wissenschaftlichen Erkenntnisse für die Praxis aufbereitet und nutzbar gemacht wurden, hat die fundierte Personaldiagnostik nur begrenzt Eingang in Organisationen gefunden (vgl. Kersting 2008, S. 13). Erkenntnisse über die Auswahlverfahren der Personaldiagnostik und deren Testverfahren werden von den Anwendern z.T. ignoriert, d.h., wissenschaftliche und eignungsdiagnostische Praxis klaffen auseinander (vgl. Koppers 2013, S. 19). Warum das Wissen um eine gute bzw. verbesserte Personalauswahl bislang wenig und nur langsam in Organisationen eindringt, ist Gegenstand der nachfolgenden Betrachtung.

\section{Ausbildung und Qualifizierung}

Als größtes Hindernis für die Umsetzung der wissenschaftlichen Erkenntnisse in die praktische Personalauswahl sieht Kanning (2011, S. 76) die mangelnde diagnostische Fachkompetenz der personalverantwortlichen Entscheidungsträger/-innen. Der Fokus liege in der wissen- 
4.4 Personaldiagnostische Prozesse und ihre Hindernisse

schaftlichen Ausbildung und nicht im praktischen Handeln. Ursache mag u.a. die Ausbildung und Qualifikation der Personalverantwortlichen sein. In der Studien von Naef (2001, S. 13 f.) war bei rund der Hälfte der 1000 befragten Organisationen eine psychologisch ausgebildete Person für die Auswahl verantwortlich. $81 \%$ zogen organisationsexterne Personen hinzu, um dies zu kompensieren. Naef schlägt Weiterbildungsmaßnahmen mit Einbindung wissenschaftlichen Know-hows nach einem abgeschlossenem Studium vor, um den wissenschaftlichen Praxistransfer zu gewährleisten. Bei einem Blick auf die angloamerikanischen Länder und ihre Hochschulstruktur zeigt sich, woran es den deutschen Universitäten mangeln könnte: Summer schools, short courses oder postgraduate studies sind Ausbildungselemente für berufspraktische Qualifikationen, die nach dem Studium oder während der Ausbildung an den Universitäten erworben werden können.

\section{Medienarbeit}

Kanning (2011) attestiert den Universitäten außerdem zu geringe Aktivitäten in Sachen Medienarbeit. Er räumt ein, 
4.4 Personaldiagnostische Prozesse und ihre Hindernisse

dass die personaldiagnostischen Erkenntnisse und Methoden in den Medien zu wenig publik gemacht würden, obgleich der Nutzen hoch sei. Er schlägt z.B. vor, dass die Diskussion um absurde diagnostische Verfahren, wie Schädeldeutung (als quasi trojanisches Pferd), Aufmerksamkeit bringen solle.

Umgekehrt sind Publikationen in Praxiszeitschriften nicht gerade förderlich für die Universitätskarriere, sodass Wissenschaftler/-innen möglicherweise davon abgehalten werden, darin zu veröffentlichen, und stattdessen dazu ermutigt werden, die akademischen Publikationsformen, wie etwa Fachzeitschriften, zu wählen. Hinzu kommt noch der Druck, in englischsprachigen Zeitschriften zu publizieren, was nicht zu einer Verbesserung des Praxis-Missenschaftstransfers beiträgt. Außerdem ist auch die Verständlichkeit dieser Beiträge für nicht ausgebildete Berufspraktiker/-innen eine Hürde.

\section{Praktische Arbeit}

Der Link von Wissenschaft und Praxis durch praktische Beratungsprojekte wird noch immer nur sehr spärlich genutzt. Parallel zur Forschung und Lehre schlägt Kanning 
4.4 Personaldiagnostische Prozesse und ihre Hindernisse

(2011) deshalb Praxiseinblicke durch Publikationen und nutzenbezogene Arbeit in Organisation vor - von denen beide Seiten profitieren. Zum einen bietet Feldforschung die Möglichkeit, sich anschließend mit den aktuellen Problemen der Praxis auseinanderzusetzen. Zum anderen verdeutlicht man den Organisationen durch Kooperationen den Nutzen wissenschaftlich fundierter Personaldiagnostik und bringt diese somit ein.

\section{Zeitliche und finanzielle Gründe}

Wissenschaftlichen Erkenntnissen zum Einstellungsinterview, wie eine Verbesserung der Personalentscheidung durch teilstrukturierte Interviews ${ }^{36}$, und andere Ergebnisse werden oft zeitliche und finanzielle Gründe entgegengestellt (vgl. Koppers 2013, S. 19). In der praktischen Personalertätigkeit werden strukturierte oder teilstruktu-

${ }^{36}$ Mit steigender Strukturiertheit des Interviews im Rahmen des Auswahlprozesses ist der Validitätszuwachs eher gering, sodass sich hochstrukturierte und stark teilstrukturierte Interviews mit zunehmender Standardisierung nicht so stark unterscheiden (vgl. Koppers 2013, S. 16). In einer Studie von Huffcutt und Arthur (1994, S. 184 f.) wurden vier unterschiedliche Strukturiertheitsgrade gemessen, und diese unterschieden sich kaum (Ergebnis: $r=.56$ für teilstrukturierte und $r=.57$ für hochstrukturierte Interviews). 
4.4 Personaldiagnostische Prozesse und ihre Hindernisse

rierte Interviews oft als zeitaufwendig, ressourcenintensiv und zu teuer abgetan. Dieses könnte fatal sein, da sich der Nutzen von strukturierten Interviews oft erst langfristig zeigt. Stephan und Westhoff (2002, S.16) untersuchten den Unterschied zwischen unstrukturierten Interviews (mit deren langfristigem Nutzen) und strukturierten Einstellungsinterviews. Es zeigte sich, dass sich die Investitionen in strukturierte Auswahlgespräche schon nach drei bis vier Monaten amortisierten.

Ausbildung und Qualifizierung, Medienarbeit, praktische Arbeit sowie zeitliche und finanzielle Gründe stehen der Verwendung wissenschaftlicher Erkenntnisse entgegen und machen deutlich, warum sie noch wenig praktische Anwendung gefunden haben. Die Darstellung der Kluft zwischen praktischer und wissenschaftlicher Anwendung in der Personaldiagnose sollte als "Schwenk" verdeutlichen, warum es für die Personaldiagnostik in Organisationen wichtig ist, dass die Erkenntnisse der Wissenschaft Eingang in die Personalauswahl finden. Dennoch implizieren die Informationen, die aus der Personaldiagnostik gewonnen werden, noch nicht die Personalentscheidung 


\subsubsection{Teilnehmende}

der Gruppe. Selbst wenn die Diagnostik valide Informationen liefert, sind diese nur die Grundlage für die Entscheidung. Um also zu einer Personalauswahl zu gelangen, müssen die Daten der Diagnostik interpretiert und ausgewertet werden. Diese Aufgabe obliegt der Gruppe. Im Weiteren soll deshalb die Zusammensetzung der Personalentscheidergruppe in Augenschein genommen werden. Wer entscheidet also in der Organisation über Einstieg, Ablehnung oder Versetzung von Bewerbern/innen bzw. wer interpretiert die diagnostischen Informationen?

\subsubsection{Teilnehmende}

Personaldiagnostische Auswahlprozesse bedingen oft allein wegen ihres Umfanges die Teilnahme unterschiedlicher Organisationsteilnehmender, um eine Entscheidung treffen zu können. Sie kommen aus verschiedenen Organisationsbereichen, wie der Personalabteilung, den Fachbereichen oder übergeordneten Leitungsfunktionen, z.B. der Geschäftsführung. Auch interne oder externe Experten/-innen für Recruiting konnten hinzugezogen werden. In Organisationen sind Entscheidungen dadurch 


\subsubsection{Teilnehmende}

bestimmt, dass es sich um soziale Prozesse in Gruppen handelt, die eine soziale Bindung an die Organisation aufweisen, die über eine Vielzahl von Entscheidungen anhält und viele Entscheidungsstufen überdauern kann (vgl. Riesenhuber 2006, S. 151). So auch bei Personalentscheidungen, denn Entscheidungen werden deshalb selten allein getroffen. Bei der Personaleinstellung und versetzung entscheiden mindestens zwei Personen als dyadische Gruppe mit. Grundsätzlich gibt es keine Höchstgrenze, aber üblicherweise sind bei mehreren Entscheidungsprozessebenen auch mehrere Organisationsmitglieder beteiligt. In Befragungen von 55 Personalleitern/-innen und 50 Betriebsräten/-innen (vgl. Femppel 2000 , S. 58 f.) gaben diese an, dass die Personalauswahl zu 90,9\%, eine kollektive Aufgabe von Personalund Fachabteilung sei. Zum Entscheidergremium der Personalauswahl können neben organisationseigenen Teilnehmenden, wie Personalreferenten/-innen, Fachvorgesetzten oder der Geschäftsführung, auch externe Personaldienstleister (z.B. mit besonderer psychologischer Eignung oder Erfahrung) gehören. 


\subsubsection{Teilnehmende}

Zu unterscheiden sind hier Entscheider/-innen und Mitwirkende. Mitwirkende können in Verwaltende (z.B. Auszubildende, die eine Erstauswahl der Bewerbermappen vornehmen) und Beobachtende eingeteilt werden (z.B. im Assessment-Center oder im Vorstellungsgespräch). Verwaltende und Beobachtende wirken am Entscheidungsprozess mit, jedoch nicht an der Auswahl und geben meist administrative Hilfestellungen.

Die Entscheider/-innen sind meist Organisationsmitglieder, deren Entscheidung auf die Endauswahl einen direkten Einfluss qua Position und/oder Eignung hat. Insbesondere das Merkmal der Eignung spielt bei der Personalauswahl eine Rolle (siehe dazu Diskussion in Kapitel 4.4). Mit dem Eintritt in die Entscheidergruppe können die sonst klaren Verantwortungszuordnungen der Organisationsbereiche in der Dynamik der Gruppe verschwimmen, denn Personalentscheidungen sind häufig interdisziplinär. Die Gruppenmitglieder kommen zusammen, weil jeder/jede Teilnehmende seine/ihre eigene Perspektive in die Entscheidung einbringt oder einbringen soll. Der/die Fachvorgesetzte kann z.B. die Fachkompetenzen beurteilen, der/die Personaler/-in gibt seine/ihre Einschätzung zum Lebenslauf ab usw. Gemeinsam beurteilt die Grup- 


\subsubsection{Teilnehmende}

pe z.B. die Motivation des/der Bewerbers/-in. Fachvorgesetzte können wegen ihrer besonderen Verantwortung im Hinblick auf die Folgen der Auswahlentscheidung sorgfältig entscheiden. Auch wenn sie im Führen von Auswahlgesprächen weniger geschult und geübt sind (vgl. Weuster 2012b, S. 56), ist die Entscheidung für sie von Tragweite, da sie im Alltag häufig mit dem/der Eingestellten zusammenarbeiten.

Allgemeines Beispiel für eine mehrstufige Einstellungsentscheidung:

Die Bewerbungsverfahren von Unternehmen beginnen häufig mit einem Stellenantrag des/der Vorgesetzten im Rahmen seiner Budgetierung bzw. der Stellenbedarfsplanung. Dabei werden die Stelleninhalte konkretisiert und als Kriterien (Anforderungsanalyse) für die Einstellung festgelegt. An jedem einzelnen Prozessschritt der Personalauswahl sind Mitglieder der Organisation beteiligt, zum Teil auch durch externe Unterstützung, z.B. durch Headhunter/-innen oder Personalvermittler/-innen. Der/die Recruiter/-in wählt die Publikationswege zur optimalen Personalgewinnung. Die (zielgruppengerechte) Stellenausschreibung ermöglicht die erste Auswahl aus 


\subsubsection{Teilnehmende}

dem Bewerberpool der einzuladenden Kandidaten/innen. Auch diese Vorentscheidung wird oft schon gemeinschaftlich mit den Beteiligten, z.B. der direkten Führungskraft, abgestimmt. Voraussetzungen für den Start des Bewerbungsverfahrens sind die Anforderungen an die Stelle. Die Stelleninhalte werden konkretisiert und der/die Wunschkandidat/-in persönlich und kompetenzbezogen bewertet. Üblicherweise werden vor dem ersten Gespräch die Bewerbungsunterlagen analysiert oder bei internen Bewerbern/-innen zusätzlich die Personalakte gesichtet. Der Personalverantwortliche führt zunächst Gespräche mit dem/der Vorgesetzten der Abteilung. Außerdem erfolgt eine Abstimmung mit der Personalabteilung und dem Betriebsrat über das Entgelt und sonstige Stellen- sowie Vergütungsbestandteile. Es wird eine Vorauswahl unter den Bewerbern/-innen getroffen, indem die Bewerbungsunterlagen analysiert werden. Auch hier sind wieder verschiedene Organisationsmitglieder involviert, sogar Praktikanten/-innen und Auszubildende können dabei mitwirken. Vorstellungsgespräche werden anschließend von dem/der Vorgesetzten und dem/der Personalverantwortlichen geführt. Die Gruppe entscheidet 


\subsubsection{Teilnehmende}

anschließend gemeinsam über die Einstellung durch Abstimmung.

Dieses Beispiel war eine Möglichkeit von vielen, einen Entscheidungsprozess zu gestalten. Auch wie die Entscheidergruppe sich zusammensetzt, ist für das Endergebnis (wer wird für die Stelle ausgewählt?) relevant. Denn wie verhält es sich, wenn unterschiedliche Hierarchieebenen in der Entscheidergruppe vertreten sind bzw. wenn sie heterogen (z.B., wenig Erfahrung vs. viel Erfahrung) besetzt ist. In der Gruppe kann dies beispielsweise dazu führen, dass Gruppenmitglieder, die eigene Bewertung in Richtung der Bewertung anderer verschieben, je höher sie die Kompetenz des/der anderen als Experten/innen einstufen (vgl. Borcherding/Schaefer 1976, S. 54). Dieser Effekt („Ich kenne mich nicht aus, also schließe ich mich den Erfahrenen an“) führt zu einem blinden Vertrauen in die Bewertung einzelner Gruppenmitglieder. Dies könnte die Gefahr bergen, sich der Meinung anderer qua allgemeine Informationen anzuschließen, indem einem Gruppenmitglied pauschal (heuristisch) attestiert wird: „arbeitet schon lange in der Firma“, „kennt sich aus mit der Personalauswahl“, „ist von der Geschäftsführung“, „kommt aus der Personalabteilung“ etc. Die Be- 


\subsubsection{Teilaktivitäten}

wertung von Personenurteilen innerhalb der Gruppe müsste gründlicher erfolgen, wird aber in der Realität auch oft durch Heuristiken ersetzt.

Mit dem Beispiel sollte verdeutlicht werden, dass in der Organisation mehrere Gruppenmitglieder an dem Einstellungsprozess beteiligt sind und sich gegenseitig beeinflussen. Im Weiteren ist deshalb von einer Personalentscheidung durch die Gruppe die Rede.

\subsubsection{Teilaktivitäten}

Das Beispiel für eine Einstellungsentscheidung aus dem vorherigen Kapitel gab bereits einen kleinen Einblick in den Auswahlprozess in Organisationen. Um welche Teilaktivitäten (in Anlehnung an den Entscheidungsprozess aus Kapitel 2.3.2) es sich speziell bei der Personalauswahl handeln kann, wird nun konkretisiert.

Neben diversen Testverfahren (siehe Kapitel 4.4) der Personaldiagnostik, wie Persönlichkeits-, Intelligenztests, Assessment-Center, Einstellungsgespräche, oder der Analyse der Bewerbungsunterlagen etc. sind Vorstellungsgespräche bzw. Interviews die häufigste personaldiagnostische Methode zur Entscheidung über die Per- 


\subsubsection{Teilaktivitäten}

sonalauswahl (vgl. Schuler 2002), obwohl ihre Validität sich in Grenzen hält (siehe Tabelle 4). In dem Interview werden zwischen dem/der Bewerber/-in und den Interviewenden der auswählenden Organisation Informationen über den/die Bewerber/-in und die Organisation ausgetauscht (vgl. Nerdinger/Rosenstiel 2011, S. 159). Neben statischen bzw. IT-basierten Verfahren ${ }^{37}$, die an Beliebtheit gewinnen, spielt das persönliche (Auswahl)Gespräch in Organisation weiterhin eine große Rolle. Mehr noch, die Personalentscheidung wird in Organisationen zumeist von dieser Prozessstufe abhängig gemacht, und ein Großteil des Entscheidungsgewichtes wird oft hierauf gelegt (vgl. Stephan/Westhoff 2002, S. 3). Das Vorstellungsgespräch ist Teil eines umfassenden Auswahlprozesses und unterscheidet sich von Organisation zu Organisation. Neben den Einstellungsinterviews ist eine Kombination der Auswahlverfahren durchaus üblich. Auch wenn unstrukturierte Einstellungsinterviews rückläufig sind, werden sie gerade in kleinen und mittle-

37 Informationstechnologien können als Hilfsmittel der Entscheidungsfindung dienen (vgl. Martin 1995, S. 29). Ob und inwieweit hierdurch die Entscheidungsfindung verbessert wird, hängt von zahlreichen situativen Faktoren sowie der Zielsetzung der Organisation ab. 


\subsubsection{Teilaktivitäten}

ren Unternehmen weiterhin gerne eingesetzt, weil z.T. die finanziellen Mittel oder die Personalkapazitäten für aufwendige oder mehrstufige Diagnoseverfahren fehlen. Jede Prozessstufe in der Personalauswahl stellt zugleich auch einen Entscheidungsschritt dar, denn die Beteiligten müssen sich für einen „Sprung“ des/der Bewerbers/-in in die nächste Auswahlstufe entscheiden. Komplexe Entscheidungen in Organisationen sind häufig Entscheidungsketten, da man, um ein bestimmtes Ziel zu erreichen, nicht mit einer Entscheidung auskommt, sondern auf eine Reihe von Entscheidungen angewiesen ist (vgl. Frey/Ochsmann 1978, S. 21). Die vielen einzelnen und organisationsspezifischen Auswahlschritte reichen von der Entscheidung, was im Anforderungsprofil steht soll, der Wahl der Zusammensetzung der Entscheidergruppe bis hin zum Ort des Vorstellungsgesprächs. Dargestellt sind in der vorliegenden Arbeit die Prozessschritte Beurteilung der Bewerber/-innen in Kombination mit der finalen Einstellungsauswahl (Entschluss) der Entscheidergruppe. 
Teilaktivitäten in der Personalauswahlentscheidung

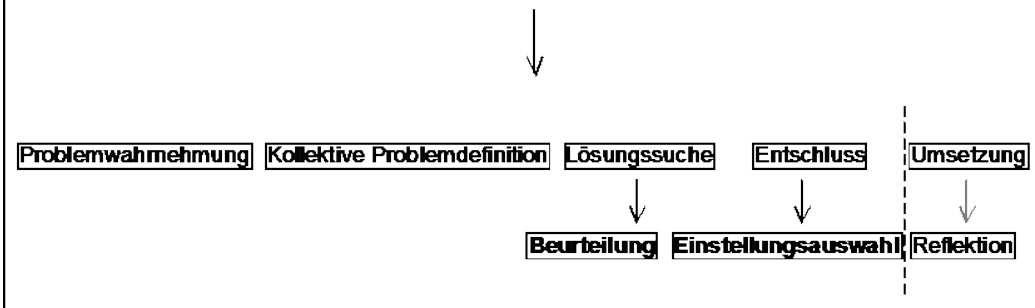

Abbildung 12: Fokus der Arbeit auf Beurteilung und Einstellungsauswahl bei der Personalentscheidung (eigene Darstellung).

Lösungssuche und Entschluss sind Teilaktivitäten der Personalauswahlentscheidung. Bevor Gruppenmitglieder hierzu kommen, ist der Personalbedarf zu planen und eine Analyse der Stelleninhalte (Anforderungsprofil) nebst Definition des Auswahlprozesses vorzubereiten. Bis dahin sind die Teilaktivitäten des Entscheidungsprozesses, Problemwahrnehmung (z.B. im Sinne der Stellenbedarfsplanung) und kollektive Problemdefinition (z.B. mit der Anforderungsanalyse), vollzogen worden (siehe Abbildung 12). Ein konkretes Auseinanderhalten der einzelnen Aktivitäten ist für das weitere Vorgehen nicht notwendig. Die Abgrenzung der Teilaktivitäten sollte ledig- 
lich verdeutlichen, dass der theoretische Entscheidungsprozess aus Kapitel 2.3.2 seine Anwendung im Personalauswahlprozess finden kann. Lösungssuche und Entschluss sind die zentralen Komponenten der Entscheidungsfindung (vgl. Jungermann 2009b, S. 9 f.). Die Auswahl der Bewerber/-innen ist gleichsam der Entschluss in der Entscheidungskette. Innerhalb der Lösungssuche verbergen sich die praktischen Akte der Personaldiagnostik, z.B. Testdurchführung und Vorstellungsgespräche. Bei dem Entschluss wird davon ausgegangen, dass es eine letzte personaldiagnostische Entscheidung der Gruppe gibt, in dem ein/eine Bewerber/-in für eine vorgegebene Stelle (die z.B. durch ein Anforderungsprofil zuvor definiert wurde) ausgewählt wird.

\subsection{Toolbox der Personalauswahl}

Um sich dem heuristischen Handeln von Gruppen bei der Personalauswahl zu nähern, sollen heuristische Entscheidungseffekte vor dem Hintergrund der adaptiven Toolbox („Werkzeugkoffer") von Gigerenzer und Todd (1999a) an dem Anwendungsbeispiel der Personalauswahl analysiert werden. Der Gedanke der Toolbox ist, 
dass jedes Individuum einen Werkzeugkoffer hat, in dem sich verschiedene Heuristiken befinden, die als Handlungshilfen dienen. Bestimmte Heuristiken können für bestimmte Entscheidungszwecke genutzt werden. Wie bei einer Werkzeugkiste eignen sich der Hammer zum Hämmern und die Säge zum Sägen. Mit der Toolbox lässt sich jedes Problem lösen, indem einfache Entscheidungsregeln (fast and frugal heuristics) angewandt werden. Heuristiken sollen dabei das kognitive Handwerkszeug des Entscheidens sein, das als kognitives Werkzeug an bestimmte Bedingungen gebunden ist (zur Lösung von Paarvergleichsaufgaben, von numerischen Schätzaufgaben oder von Kategorisierungsaufgaben) (vgl. Reimer et al. 2007, S. 26). Gigerenzer und Todd (1999a, S. 30) definieren den Begriff „adaptive Toolbox“ so: „(A) collection of spezialized cognitive mechanisms that evolution has built into the human mind for specific domains of inference and reasoning, including fast and frugal heuristics." Jedes Individuum trägt dabei die Anlagen bereits in sich (,with just the things at hand"), die auf Wahrnehmungs- und Erinnerungsprozesse beruhen. 
Die Toolbox der Personalauswahl ist gefüllt mit Heuristiken, die als Entscheidungswerkzeuge dienen sollen. In Bezug auf die Forschungsfrage, ob Heuristiken auch analog auf kollektiver Ebene genutzt werden, stellt sich die Frage, ob sich die Heuristiken aus der Toolbox dazu eignen, auch von Gruppen angewandt zu werden.

Auch gemeinsam handelnde Teilnehmende benötigen ein Handwerkszeug des Entscheidens. Ihr Entscheidungshandeln ist komplex, denn neben der inhaltlichen Komplexität kommt hinzu, dass die Werkzeuge der Gruppe durch soziale Entscheidungsprozesse miteinander verbunden sind. Um bei der Analogie des Werkzeugkastens zu bleiben: Man stelle sich eine verzahnte Produktionsmaschine anstelle eines einfachen Werkzeugkastens vor. Informationen fließen nur, wenn die Verkettung der Maschinenteile miteinander optimal ist. Den Schmierstoff stellen dabei die sozialen Prozesse dar. Konflikte können die sozialen Austauschmechanismen unterbinden, z.B. einen adäquaten Informationsaustausch. Die Maschine produziert nur angemessene Entscheidungen, wenn das Zusammenspiel klappt. Jedoch ist dabei nicht vonnöten, dass alle Informationen detailliert ausgetauscht werden. Es geht um die für die Gruppe 
4.5 Toolbox der Personalauswahl

praktikabelste Form des Informationsaustausches mittels Anwendung der Toolbox für eine adäquate Gruppenentscheidung.

Die Heuristiken der Gruppe entfalten ihre Wirkung durch Gruppendiskussion und Informationsaustausch, sodass Heuristiken den Entscheidungsprozess nicht lähmen, sondern von der Gruppe gestaltet werden. Denn auch eine Gruppe kann von der Komplexität oder der Fülle an Eindrücken oder Informationen überfordert sein (information overload) und benötigt Mechanismen, die sie trotzdem handlungsfähig bleiben lassen (vgl. Reimer et al. 2007, S. 8). Auch wenn jedes Gruppenmitglied alle Informationen preisgeben könnte, käme die Gruppe oft in Zeitnot oder Stress. Wie Individuen kann auch eine Gruppe mittels Heuristiken Anpassungsmechanismen schaffen. Die sozialen Prozesse der Gruppenmitglieder moderieren diese heuristischen Abkürzungseffekte, z.B., indem Einzelne die Gruppenleitung und damit die Prioritätenlenkung übernehmen. Das Übersehen bzw. Ignorieren von Informationen oder eine falsche Prioritätensetzung sind dabei nur zwei Entscheidungsfehler, die auftre- 
ten können. ${ }^{38}$ Die adaptive Toolbox enthält Werkzeuge zum Schlussfolgern, zur Bildung von Präferenzen und Entscheidungen bereit (vgl. Gigerenzer/Marewski 2013, S. 232). Die gleichen Mechanismen benötigt auch die Gruppe, um ihr Verhalten zu lenken, ohne der Komplexität der Aufgaben zu erliegen. Die adaptive Toolbox des heuristischen Handelns existiert somit auch in der Gruppe (vgl. Reimer et al. 2007, S. 9 f.).

Die adaptiven Werkzeuge stellen Strategien der Informationsverarbeitung zur Verfügung und bestehen in Mechanismen, die der Vergangenheit bereits erfolgreich evaluierten. Evolutionsbiologisch sind heuristische Strategien an die Umwelt angepasste Verhaltensweisen. Heuristiken werden damit dem Modell der bounded rationality (vgl. Hoffrage/Reimer 2004) zugeordnet und auch als fast and frugal heuristics bezeichnet, weil der Forschungsansatz von einem angepassten einfachen menschlichen Verhalten ausgeht (siehe Abbildung 5). Die Aufgabe der Heuristiken ist es, Informationen schnell abzurufen, ohne

${ }^{38}$ Auch das Thema Führung kann bei Heuristiken in Gruppen eine entscheidende Rolle spielen, da Führung zugleich die thematische Lenkung von Heuristiken bedeuten kann. 
zu simplifizieren oder die Komplexität außer Acht zu lassen. Der Ansatz der ABC-Gruppe (Todd, Gigerenzer und $A B C$ 1999) geht sogar so weit, ein Umdenken in der Positionierung des Menschen in der Welt zu fordern (vgl. Woike 2007, S. 136). Es ist das Konzept des Homo heuristicus (entwickelt von Todd und Brighton 2009) und soll der Sichtweise Rechnung tragen, dass die Grenzen der menschlichen Verarbeitungs- und Entscheidungsfähigkeit als ökologische Anpassungsstrategien angewandt werden.

Dass Heuristiken auch in der Personalauswahlsituation (wie in den meisten anderen Entscheidungssituationen) angewandt werden, erscheint denklogisch nachvollziehbar. Denn die Personalauswahl ist die komplexe Aufgabe, aus einer Menge an Informationen über verschiedene Bewerber/-innen (persönlicher Eindruck, Lebenslauf, Ergebnisse der gestellten Aufgaben etc.) einen oder mehrere auszuwählen und dies mit den Anforderungen der Organisation (Anforderungsprofil, Gehaltsbänder/Tarif, Befristungen etc.) zu vergleichen. Bei einer rein rational geprägten Entscheidung müsste eine Entscheidergruppe zum Beispiel Folgendes berücksichtigen: 
- Erfüllt der/die Bewerber/-in das Anforderungsprofil und andere organisationale Anforderungen (Diagnose)?

- Wie wahrscheinlich ist der Organisationserfolg des/der Bewerbers/-in nach der Einstellung (Prognose)?

- Wie gut passt er/sie sich an die Organisation, deren Umgebung und ihre Beteiligten an (Passung)?

Die diagnostische Personalauswahl der Gruppe muss dabei u.a. folgende Informationen wahrnehmen und beurteilen:

- Gezeigte Verhaltensweisen (verbal und nonverbal)

- Fachkenntnisse, Fertigkeiten und Fähigkeiten

- Unterlagen, wie Lebenslauf oder Testergebnisse

Aus der diagnostischen Wahrnehmung der Gruppenmitglieder ergeben sich (Zwischen-)Urteile, die aber auch von indirekten und subjektiven Hinweisreizen abgeleitet werden können. Es handelt sich bei der Personaldiagnostik um eine Momentaufnahme, und ihre Gesetzmäßigkeiten variieren aufgrund subjektiver und situativer Einflüsse. Wechselnde Gruppenmitglieder können auch 
4.5 Toolbox der Personalauswahl

andere Entscheidungen treffen. Dies hängt damit zusammen, dass Wahrnehmungs-, Informations- und Urteilprozesse immer selektiv und subjektiv erfolgen und maßgeblich durch die Lernprozesse und Erfahrungen geprägt sind (vgl. Kanning 2012, S. 33), sodass absolute Rationalität in der Personalauswahl nicht gelingen kann. Außerdem sind die Mitglieder nicht in der Lage, alle verfügbaren Informationen z.B. während des Einstellungsinterviews aufzunehmen und optimal zu gewichten (Anderson/Shackleton 1990, S. 70; zit. n. Koppers 2013, S. 33). Damit gelingt es nicht, rationale Entscheidungen bei der Personalauswahl zu treffen.

Um dieser Informationsflut Herr zu werden, werden heuristische Mechanismen angewandt, die die Entscheider/innen ergreifen. Heuristiken treten in der Personalauswahl nicht nur wegen der Informationsgrenzen auf, sie sind für die gemeinsame Anwendung in der Gruppe auch wegen der Grenzen der Antizipation (Abschätzen der Konsequenzen) sowie der Begrenzung der Alternativenauswahl (nicht alle Alternativen sind bekannt) (siehe Kapitel 4.3) von Nutzen.

Bei Personalentscheidungen handelt es sich um komplexe Entscheidungen, die selten von Einzelnen, sondern in 
4.5.1 Die Kausalität von Information und Prognose mehrstufigen, seriellen Prozessen (u.a. Analyse der Bewerbungsunterlagen, Vorstellungsgespräche, Assessment-Center) von Kollektiven getroffen werden. Die finale Entscheidung basiert oft auf einer Serie von Interviews oder anderen personaldiagnostischen Prozessen (vgl. Fifić/Gigerenzer 2014, S. 1). Hierbei kann es zu vielfältigen Einflüssen kommen, die die Entscheidung nicht einheitlich ausfallen lassen, da, wie zuvor beschrieben, eben nicht alle relevanten Informationen oder Argumente berücksichtigt oder Gegenargumente aus sozialen Erwägungen bewertet werden.

Bevor auf einzelne Heuristiken eingegangen wird, soll im nächsten Kapitel die Verbindung von Bewerberinformationen mit der Prognose der künftigen beruflichen Leistung der Bewerber/-innen diskutiert werden. Diese Verknüpfung soll zur Bewertung der heuristischen Entscheidung beitragen.

\subsubsection{Die Kausalität von Information und Prognose}

Personalentscheidungen werden häufig nach individuellen Kriterien getroffen, z.B. Müll im Auto oder nicht gebü- 
4.5.1 Die Kausalität von Information und Prognose

gelte Kleidung als Indikatoren für fehlende Zuverlässigkeit und als Schlussfolgerung auf mangelnde Sorgfalt im Beruf. Diese und andere Faustregeln bzw. intuitive Auswahlnormen werden zu subjektiven „Wahrheiten“ bei der Personalentscheidung als Auswahlregeln für Einzelne oder Gruppen. Dennoch greifen Gruppenmitglieder auf so einfache Auswahlregeln, wie "Gute Bewerber/-innen beherrschen die Grammatik und räumen ihr Auto auf!", zurück. Dass allein die Grammatik eines/einer Bewerbers/-in als Einzelkriterium für die Prognose seines/ihres beruflichen Erfolges nicht taugt, versteht sich von selbst. Dennoch gibt es diese Fehlschlüsse zuhauf, und sie beruhen auf den Erfahrungen Einzelner und haben wenig mit einer rationalen Personalauswahl zu tun. Intuitive Entscheidungsregeln legt jeder/jede für sich selbst fest, sie unterliegen der eigenen „Wertewelt", also den Werthaltungen und Erfahrungen, die Personen im Laufe ihres Lebens für sich validiert haben. Sie sind nicht für jeden als Entscheidungskriterium nachvollziehbar oder geeignet. Heuristisches Entscheidungsverhalten ist intuitiver Natur und speist sich aus emotionalen und kognitiven Elementen. 
4.5.1 Die Kausalität von Information und Prognose

In der Personalauswahl wird die künftige berufliche Leistungsfähigkeit häufig auf Basis der Bewerberinformationen inkl. der Diagnoseergebnisse aus Test, Interviews etc. abgeleitet. Doch können (Test-)Ergebnisse und Bewerberinformationen fehlinterpretiert werden, indem die falsche Bewertung auf die Prognose umgelegt wird. Die erhoffte Prognose wird deshalb unvollständig oder fehlerhaft eingeschätzt (vgl. Riesenhuber 2006, S. 44). Die Information wird anhand eigener Referenzpunkte (Normen) interpretiert und bei der Entscheidung mit der künftigen Leistungsprognose verknüpft (siehe Kapitel 3.3.1). Die Beurteilung der Leistung hängt also mit den Referenzpunkten der Teilnehmenden zusammen. Worauf nehmen sie bei der Bewertung ihrer Entscheidung Bezug (vgl. Riesenhuber 2006, S. 65)? Es stellt sich die Frage, ob die subjektiven Referenzpunkte sich als Interpretationsmaßstab für die Information eignen können. Damit Referenzpunkte sich für eine Entscheidung eignen, müssen diese erst einmal validiert werden bzw. durch Lernprozesse evaluiert worden sein.

Beispiel: Ein Bewerber ist Leistungssportler, und deshalb wird inm automatisch unterstellt, dass er die gleiche hervorragende Leistung auch im Beruf erbringen wird. Die 
4.5.1 Die Kausalität von Information und Prognose

Entscheider/-innen erachten sportliche Leistung als Referenzpunkt zum Berufserfolg. Diese Ableitung von einem Kriterium (Leistungssport) auf das nächste (Berufserfolg) ist ein Umlagefehler, der zu einer Heuristik wird. Dieses Phänomen kann als heuristischer (Fehl-)Schluss bezeichnet werden, d.h. als eine nicht kausale Ableitung der Information auf die Prognose. Im Gegensatz zur Sichtweise, dass eine heuristische Entscheidung nicht zwingend fehlerhaft sein muss, ist beim heuristischen Fehlschlusses die heuristische Entscheidung fehlerhaft. Dieser Ansatz entspricht auch der Sichtweise des heuristic and biases program von Kahneman und Tversky (1974). Der heuristische Fehlschluss charakterisiert begrenzt rationales Entscheiden als irrational und defekt.

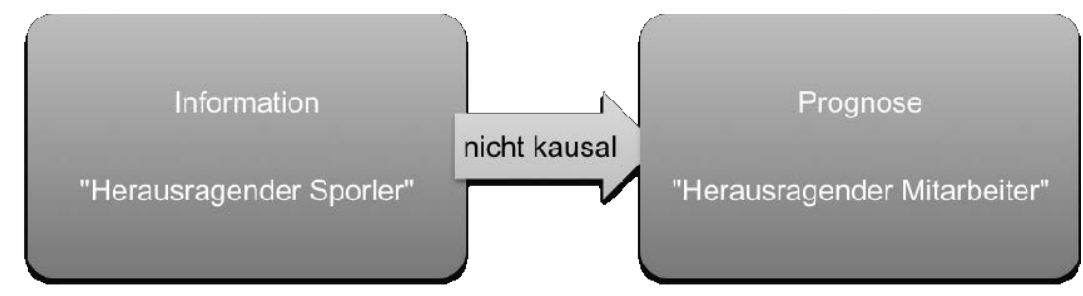

Abbildung 13: Beispiel für einen heuristischen Fehlschluss von einer Information auf eine Prognose (eigene Darstellung). 
4.5.1 Die Kausalität von Information und Prognose

Konkret: Ein/eine Bewerber/-in stellt nach seiner Einstellung vielleicht als ein/eine guter/gute Mitarbeiter/-in, nicht aber als ein besonders herausragender Kopf dar (trotz möglicherweise herausragender Ergebnisse auf sportlichem Gebiet). Auch hier findet die Kausalität, wie in Kapitel 3.3.1, Eingang, d.h., die kausale Verlinkung von Information und Prognose kann auch bei der Personalauswahl fehlgehen. Eine Information lässt nicht unbedingt den Rückschluss auf eine Prognose zu. Dennoch wird oft kein Unterschied zwischen der Vorhersage eines künftigen Ereignisses und der Beurteilung der gegebenen Informationen gemacht (vgl. Kahneman 2011, S. 235). Dieser Fehlschluss kann z.B. auch auftreten, wenn es um Ergebnisse von Tests geht. Die Gruppenmitglieder, die über die Einstellung entscheiden, leiten aus den Testergebnissen den künftigen beruflichen Erfolg ab. Häufig wird die Validität dieser Tests nicht hinterfragt und mit den Gegebenheiten der späteren Realität nicht gespiegelt.

Den Fehlschluss, von der Bewerberinformation auf den Handlungserfolg abzuleiten, lässt die Prognose (als Entscheidungsgrundlage) heuristisch werden. Grundsätzlich 
4.5.1 Die Kausalität von Information und Prognose

sollen die Informationen der Erleichterung des Prozesses der „Einstellung“ dienen, aber nicht jeder Test und jede Informationen sind valide Instrumente für jeden Beruf. Unter Umständen sind die Kompetenzen, die in dem Test erfragt oder simuliert werden, völlig unbrauchbar für die individuelle berufliche Organisationspraxis. Das Vertrauen in die Instrumente der Personaldiagnose kann nicht die ganzheitliche Entscheidung durch weitere Entscheidungsmethoden im Auswahlprozess ersetzen.

Ein weiteres Beispiel gibt Kahneman (2011, S. 232 f.). Er konstruiert folgenden Fall zur Befragung von Probanden: Eine Studentin im vierten Studienjahr einer staatlichen Universität konnte im Alter von vier Jahren flüssig lesen. Was ist ihr Notendurchschnitt?

Die statistische Antwort auf diese Frage wäre die durchschnittliche Note im Bundesdurchschnitt aller Studenten/innen im vierten Studienjahr. Jedoch ignorierten die Probanden die Wahrscheinlichkeit und beantworteten die Frage mit einer besseren Note statt der Bundesdurchschnittsnote. Sie stellten eine nicht kausale Verknüpfung von Informationen (die Lesefähigkeit) und der Prognose (einem guten Notendurchschnitt) her. Die tatsächliche Vorhersagequalität der Informationen „frühe Lesefähig- 
4.5.1 Die Kausalität von Information und Prognose

keit" ist gering, jedoch assoziiert das Gedächtnis diese Information mit der Vorhersage. Es fehlen die Vergleichsgruppe und die Vergleichsnorm.

Das Problem mit der fehlenden Vergleichsgruppe stellt sich auch in der Personalarbeit dar. Insbesondere, wenn es um Leistungsindikatoren, wie Schulnoten, Abschlüsse oder Auslandsaufenthalte, geht, gelingt es den Entscheidern/-innen im Auswahlprozess selten, diese anderen gegenüberzustellen. In einigen Studiengängen wird die Abschlussnote als Heilsbringer für den beruflichen Erfolg angesehen. In der deutschen Juristenausbildung erhalten z.B. nur die vermeintlich Besten Stellen in internationalen Großkanzleien und der deutschen Justiz, diejenigen also, die ein vollbefriedigend ( $>9$ Punkte und somit ein sog. Prädikatsexamen) vorzuweisen haben. Ungeachtet der künftigen Tätigkeit wurde diese ungeschriebene „Norm“ zur Messlatte für künftige Leistungsfähigkeit, auch wenn sie sich als heuristischer Fehlschluss entpuppen kann. Ist denn beispielsweise ein mit Prädikatsnoten ausgezeichnete/r Richter/-in wegen seiner/ihrer Note im Studium ein/eine guter/gute Richter/-in oder aber, weil er/sie parteienunabhängig ist und die Parteien durch geschickte Bemühungen vergleichen kann? Noch augenfälliger wird 
4.5.1 Die Kausalität von Information und Prognose

es beim/bei der evangelischen Pastor/-in. Derjenige/diejenige, der/die einen sehr guten Studienabschluss vorweisen kann, bekommt eine Pfarrei mit Gemeinde. Ist diese Person nur deshalb ein/eine guter/gute Pastor/-in mit all den notwendigen menschlichen Fähigkeiten und Aufgaben (wie Begräbnisse, Hochzeiten etc.), nur weil er/sie sehr gute Abschlussnoten hatte?

Verhilft denn die computerunterstützte Personalauswahl zur einer objektiven Personalauswahl, die den künftigen beruflichen Erfolg vermitteln kann? Die Tendenz zum Einsatz von computergestützten Informations- und Expertensystemen bei der Personalauswahl steigt (vgl. Schüßler 1993, S. 105 f.) und ist eine Möglichkeit zur Verbesserung der menschlichen Informationsverarbeitung, die stärker vernunftbasiert sein soll. Obwohl mit ihr ein zusätzlicher organisationaler Aufwand einhergehen kann (Eingabe, Bewertung und Auswertung der Daten), gaben in einer Studie von Naef (2001, S. 12 f.) mit 1000 Organisationen Personalentscheider/-innen mehrheitlich (57\%) an, vermehrt computergestützte Verfahren zur Personalauswahl einsetzen zu wollen, um die Prognosequalität zu verbessern und mehr Objektivität zu erzielen. 
4.5.1 Die Kausalität von Information und Prognose

Außerdem könnte eine computergestützte Rekrutierung neben der Personalauswahl auch für die interne Weiterbildung und Personalentwicklung genutzt werden. Jedoch gehen soziale Bewertungskomponenten, wie individuelle Präferenzsysteme, schwer mit einer Quantifizierung von Personalauswahlkriterien einher. Gemeint sind zwischenmenschliche Aspekte, wie Teamfähigkeit, Sympathie, Anpassungsfähigkeit oder Begeisterungsfähigkeit. Denn gerade „zwischenmenschliche“, intuitive Aspekte sind gleichfalls wichtig für eine erfolgreiche Zusammenarbeit. Genauso wie die Ergebnisse der computergestützten Rekrutierung, die sich nur auf Noten oder andere messbare Kategorien konzentriert. Der Personalauswahlprozess ist demnach gespickt mit Heuristiken, ob diese jedoch Fehlschlüsse sind, hängt von zahlreichen Faktoren $a b$, und selbst wenn der heuristische Fehlschluss die Entscheidungsqualität konterkariert, muss sich daraus nicht zwingend eine kausale Ableitung zum tatsächlichen Handlungserfolg ergeben (künftige Leistung des/der Bewerbers/-in). 
4.5.2 Heuristische Entscheidungsregeln in der Personalauswahl

\subsubsection{Heuristische Entscheidungsregeln in der Personalauswahl}

Im vorherigen Kapitel konnte dargestellt werden, dass eine heuristische Entscheidung nicht notwendigerweise kausal zum anschließenden Verhalten sein muss. Im Folgenden soll betrachtet werden, wie Heuristiken in der Personalauswahl wirken. Hierzu werden die drei heuristischen Grundregeln von Gigerenzer und Todd (1999a, S. $23 \mathrm{f}$.) auf die Personalauswahl angewandt. Bereits in Kapitel 3.3.2, angelehnt an die Analyse „Entscheidungsheuristiken in Gruppen“ von Reimer et al. (2007), wurden die Grundregeln vorgestellt und die Übertragbarkeit auf Gruppen diskutiert.

Zunächst einige allgemeine Beispiele für eine heuristische Personalauswahl:

- Wir stellen nur die Besten ein.

- Wenn eine Person nicht ehrlich und vertrauenswürdig ist, spielt alles andere (z.B. die Fähigkeiten oder das Wissen der Person) keine Rolle (vgl. Gigerenzer/Marewksi 2013, S. 239). 
4.5.2 Heuristische Entscheidungsregeln in der Personalauswahl

- Personalentscheidungen treffen wir aus dem Bauch heraus. Schließlich müssen wir ja gut zusammenarbeiten.

- Kandidaten/-innen mit sportlichem Hintergrund werden bei uns gern genommen.

- Ohne Auslandserfahrung geht gar nichts.

Die Heuristik „Wir stellen nur die Besten ein" soll nun als Anwendungsbeispiel für eine Heuristik bei Personalauswahlentscheidungen vorgestellt und in den drei Entscheidungsregeln (Informationssuche, Informationsstopp und Entscheidung) abgebildet werden:

\section{Informationen suchen}

Bei der Heuristik „Wir stellen nur die Besten ein“ liegt die Suche nach den Besten im Auge des Betrachters. Für die Entscheidung, wer der/die Beste ist, legen die Entscheider/-innen Referenzpunkte und Normen für sich fest. Mit der Heuristik definieren sie einen oder mehrere zentrale Auswahlkriterien (Eigenschaften, Fähigkeiten, Fertigkeiten, Erfahrungen etc.) für sich als das „Beste“. Die Suchregel basiert vor dem Hintergrund der Organisa- 
4.5.2 Heuristische Entscheidungsregeln in der Personalauswahl

tionsumgebung auf Erfahrungen und Wissen in Bezug auf die Personalauswahl. Die Entscheider/-innen könnten zum Beispiel der Meinung sein, dass die besten Bewerber/-innen diejenigen sind, die im Ausland gearbeitet oder auf einer Privatuniversität studiert haben, spezielle Stationen im Lebenslauf aufweisen, wie ehrenamtlich gearbeitet oder die höchsten Werte im Einstellungstest gehabt zu haben. Mit ihren subjektiven Suchregeln im Hinterkopf würden sich die Organisationsteilnehmenden in Richtung Entscheidung bewegen, aber mit einer Informationsaufnahme, die nach Kriterien für die Bestätigung ihrer subjektiven Regeln sucht. Wie beim Halo-Effekt als dem klassischen Entscheidungsdefekt der individuellen Personalauswahl lässt die Heuristik auch nur einen verengten Blick auf die Kriterien für die „Besten“ zu. Andere wichtige und entscheidungsrelevante Bewerberfaktoren würden aus dem Wahrnehmungsfokus ausgeblendet.

Diese Verzerrung der Bewerberauswahl zeichnet zunächst das Bild einer defekten Personalauswahl. Denn wo noch nicht alle Informationen vorliegen, kann keine rationale Auswahl getroffen werden, die einem/einer Bewerber/-in gerecht wird. Doch dies muss nicht automa- 
4.5.2 Heuristische Entscheidungsregeln in der Personalauswahl

tisch zu einer fehlerbehafteten Entscheidung führen, da auf den zweiten Blick intuitiv die richtigen Informationen für die zu treffende Entscheidung selektiert werden könnten. Denn nicht jede Personalentscheidung bedarf aller Informationen: Die Beschränkung der verfügbaren Informationsflut kann auch ein Gewinn für die Personalauswahl sein, wenn sie an die Informationsumgebung und Aufgabenumwelten angepasst sind (vgl. Reimer et al. 2007, S. 26). Das heißt, die Entscheidung fügt sich in die Organisation ein und wird in die Gegebenheiten integriert. Hierzu gehört auch, ob die Bewerber/-innen in die Arbeitsumgebung passen. Dass Heuristiken angewendet werden und sie erfolgreich sind, konnte von einigen Autoren/-innen dargestellt werden, wie zum Beispiel Gigerenzer und Todd (1999a, S. 27 f.). Sie mischen der menschlichen Entscheidungsfähigkeit dabei ein gutes Stück Optimismus bei, wohingegen die Forscher des heuristic and biases program, Kahneman und Tversky (1974), das begrenzt rationale Entscheiden als irrationales und von Defekten gezeichnetes Verhalten beschreiben. 
4.5.2 Heuristische Entscheidungsregeln in der Personalauswahl

\section{Beenden der Informationsaufnahme}

Die Suche nach Informationen über den/die Bewerber/-in gerät irgendwann an ihre (kapazitätsbezogenen) Grenzen, sodass sie beendet werden muss. Dies ist beispielsweise dann der Fall, wenn die Suche aus zeitlichen Gründen gestoppt wird, weil eine schnelle Einstellungsentscheidung erforderlich ist. Dazu gehört auch, dass die Suche nach weiteren Bewerbern/-innen abgebrochen wird, z.B. durch keine neue Stellenausschreibung oder zusätzliche Rückfragen an den/die Bewerber/-in. Die Gruppenmitglieder verwenden dann für ihre Entscheidung die vorhandenen Informationen und schränken den Auswahlradius der Informationssuche ein. Entweder, weil sie sich bereits für einen/eine Bewerber/-in entschieden haben oder meinen, über ausreichend Wissen über die Bewerber/-innen zu verfügen.

Unter Umständen werden infolge des abgebrochenen Auswahlprozesses potenziell geeignete Bewerber/-innen außer Acht gelassen, z.B. weil Bewerberinformationen nicht in diesen Auswahlprozess integriert werden. Wann die Informationsaufnahme für eine abschließende Bewertung beendet wird, hängt u.a. auch von der Qualität der 
4.5.2 Heuristische Entscheidungsregeln in der Personalauswahl

Stellenbewerbenden ab (vgl. Gigerenzer/Marewski 2013, S. 232). Vereinfacht gesprochen, wird der Ausschreibungs- und Auswahlprozess umso früher beendet, je größer die Passung der potenziellen Bewerber/-innen mit der Stellenanforderung ist.

Auch die Analyse, ob die vorliegenden Bewerbungen die größtmögliche Passung mit dem Stellenprofil haben, ist ihrerseits wieder eine heuristische Auswahl. Denn die Gruppenmitglieder müssen entscheiden, welches Anforderungsprofil (z.B. laut Stellenprofil) erfüllt sein muss, um aus einer Menge an Bewerbenden einen/eine Kandidaten/-in auszuwählen. ${ }^{39}$ Als heuristische Entscheidungsregeln kommen hierbei einige in Betracht. Hierzu zählen auch Heuristiken, die rationales Wissen überlagern können. Wenn z.B. als Grundlage der Einstellung bestimmte

${ }^{39}$ Hierbei stellt sich die Frage, wie Anforderungsprofile beschaffen sein müssen, um ihren Anwendern eine valide Basis zu ermöglichen, aus der sie ableiten können, wie Aufgabe, Verhalten und Eigenschaften im Einklang mit der Stelle zu erbringen sind. Schuler (2002, S. 135 f.) und Weuster (2012b, S. 37 f.) geben Empfehlungen für die Erstellung eines Anforderungsprofils als Analyseelement bei der Personalauswahl. Inhalte können u.a. sein: fachliche Kenntnisse und Qualifikationen, Berufs- und Führungserfahrung, Persönlichkeit, Fertigkeiten und Kompetenzen, stellenrelevante Verhaltensanforderungen etc. (vgl. Koppers 2013, S. 18). 
4.5.2 Heuristische Entscheidungsregeln in der Personalauswahl

Anforderungen genannt sind, wie Berufserfahrung in der Branche, entscheiden sich die Gruppenmitglieder dagegen, diesem Kriterium den Vorzug zu geben. Stattdessen nehmen sie den/die Bewerber/-in, der/die gut ins Team passt und eine/eine netter/nette Kollege/-in sein könnte. Liebe, Freundschaft, Emotionen etc. gehören zu den wenig kalkulierbaren Entscheidungsthemen, die sich mathematisch nicht erfassen lassen (vgl. Gigerenzer/Todd 1999a, S. 15), genauso wenig wie Teamfähigkeit, Sympathie und Anpassungsfähigkeit. Ein anderes Beispiel, in dem die Vorgaben der Organisation ignoriert werden: Die Gruppenmitglieder entscheiden sich bewusst für jemanden, der - umgangssprachlich ausgedrückt - die eigene Macht nicht „untergräbt“, bzw. jemanden, der einem nicht das „Wasser abgräbt“ oder am „Stuhlbein sägt“. Diese heuristischen Überlegungen blenden die rationale Entscheidung aus und ziehen Präferenzen, wie eigene Karrierevorstellungen oder hierarchische Einflüsse, vor.

Bei Heuristiken in der Personalauswahl kann es sich um Vorentscheidungen handeln, die ein schnelles Urteil über den/die Bewerber/-in treffen, ohne dass der Prozess schon abgeschlossen ist. Dies geschieht häufig bereits in 
4.5.2 Heuristische Entscheidungsregeln in der Personalauswahl

einer sehr frühen Phase des Auswahlprozesses, nämlich im Einstellungsinterview. Untersuchungen mit erfahrenen Interviewern/-innen in einem unstrukturierten Interview ergaben eine Entscheidung für oder gegen einen/eine Bewerber/-in bereits nach vier Minuten (vgl. Eder/Buckley 1988, S. 94; zit. n. Weuster 2012b, S. 52). In unstrukturierten Interviews wird die Entscheidung also bereits zu einem sehr frühen Zeitpunkt getroffen, dies weist somit auf einen eher intuitiven und subjektiv geprägten Entscheidungsprozess hin (vgl. Koppers 2013, S. 25).

Eine frühe Vorentscheidung in der Auswahl geht zugleich auch mit einem Beenden der Informationsaufnahme einher. Denn, wer sich schon entschieden hat, benötigt keine weiteren Informationen. Wenn der/die „Beste“ bereits innerhalb der ersten Minuten ausgewählt wurde, wird die Aufnahme der vorhandenen Informationen gestoppt (Cutoff-Verhalten) und das Zulassen von weiteren notwendigen oder überflüssigen Bewerberdaten behindert oder ausgeblendet. Bei Einstellungsinterviews findet bereits im Gespräch eine Schnelldiagnose statt. Sie führt insbesondere bei unterdurchschnittlichen Bewerbern/-innen zu einer Abschwächung oder sogar Beendigung der Eig- 
4.5.2 Heuristische Entscheidungsregeln in der Personalauswahl

nungsdiagnose (vgl. Weuster 2012b, S. 52), die zugleich auch richtungsweisend für das weitere Interview sein kann. Das Verhalten der Interviewteilnehmenden ändert sich durch eine Vorabentscheidung und beeinflusst das Gespräch (unbewusst). Informationen werden nach einer Vorabentscheidung gefiltert und es wird auch nach Argumenten im Bewerberverhalten gesucht, die die vorherige Schnellentscheidung untermauern.

\section{Entscheidung}

In diesem Stadium des Prozesses ist die Informationsaufnahme beendet und die Entscheidung des einzelnen Organisationsmitglieds ist entscheidungsreif. Es soll der/die Beste eingestellt werden, und mit diesem Entscheidungsziel soll die Entscheidung getroffen werden: Das Abwägen und der Austausch beginnen.

Die Entscheidungsfindung auf Grundlage der vorhandenen Informationen über den/die vermeintlich besten/beste Bewerber/-in mündet in eine Entscheidungsregel, die das Gruppenmitglied zunächst für sich bestimmt. Es selektiert die Bewerberinformationen, um zu einer Entscheidung zu gelangen, die den Erwartungen an 
4.5.2 Heuristische Entscheidungsregeln in der Personalauswahl

den/die „Besten“/,Beste“ entsprechen. ${ }^{40}$ Zugleich spielen auch soziale Erwartungen an die Entscheidung eine Rolle, d.h., das Gruppenmitglied muss sich daran messen lassen, wen auch die Organisation und ihre Mitglieder für den/die Besten/Beste halten. Neben den Einflüssen der Organisation beeinflussen auch die anderen Mitglieder die Entscheidung. Hier kann es zu Konflikten und Rechtfertigungsdruck kommen.

Heuristiken sind einfache Regeln von Gruppen und Individuen, die bei der Entscheidung angewandt werden. Heuristiken lassen sich wegen ihrer hohen ökologischen Validität und Rationalität nicht in ein Korsett pressen, aber sie können einen Beitrag zur Erkenntnisgewinnung

${ }^{40}$ Dabei ist es nicht unüblich, viele Stellenbewerber/-innen zum Entscheidungsinterview zu laden, um damit die Objektivität des Prozesses und die eigene Glaubwürdigkeit zu untermauern (,je mehr Bewerber/-innen, desto glaubwürdiger/objektiver der Einstellungsprozess als Entscheidungsregel"). Mehr Bewerber/-innen verbessern zwar nicht die Auswahlobjektivität, aber wiegen die Entscheider/innen in der Sicherheit, eine gewisse Menge an Bewerbern/-innen beurteilt zu haben. Auch dies kann als Suchregel ausgelegt werden, da für den/die Bewerber/-in ohne dieses Auswahlkriterium (z.B. mindestens fünf Bewerber/-innen sind für die finale Selektion nötig), keine finale Auswahl möglich wäre. Wie sinnvoll solche Entscheidungsregeln sind, sei dahingestellt. Aber es liegt in der Natur der Heuristiken, ihren Anwendern/-innen gewisse Eigenheiten zu gewähren. 
4.5.2 Heuristische Entscheidungsregeln in der Personalauswahl

leisten, um menschliches Verhalten zu verstehen, das in einer sozialen Umwelt stattfindet. In dem Artikel „What we have learned so far" haben Gigerenzer und Todd (1999b, S. 357) ihre Einstufungen von Heuristiken erweitert. Sie differenzieren darin die fast and frugal heuristics nach One-Reason Decision Making, Ignorance-Based Decision Making und Satisficing (vgl. Gigerenzer/Todd 1999b, S. 358 f.). Ergänzt werden soll diese Aufzählung durch die Verfügbarkeitsheurstik von Kahneman und Tversky (1974).

- One-Reason Decision Making (vgl. Gigerenzer/Todd 1999a, S. 14; Gigerenzer/Todd 1999b, S. 358): Bereits ein wichtiger Grund genügt, um eine Entscheidung zu treffen. Ein eindrucksvolles Beispiel gibt Gigerenzer (vgl. 2009a, S. 7) mit der Frage, die sich Charles Darwin stellte, ob er heiraten solle oder nicht. Aus den zahlreichen Argumenten hätte bereits eines ausgereicht, um sich für oder gegen eine Heirat zu entscheiden (z.B. nicht allein sein).

- Ignorance-Based Decision Making (vgl. Gigerenzer/Todd 1999b, S. 358): Informationen, die u.U. 
4.5.2 Heuristische Entscheidungsregeln in der Personalauswahl

wichtig für die Entscheidungsfindung wären, werden ignoriert.

- Satisficing: Die Gründe für die Entscheidungsfindung und die Entscheidung selbst werden als angemessen angesehen (vgl. Gigerenzer/Todd 1999b, S. 358).

- Die Verfügbarkeitsheurstik von Kahneman und Tversky (1974) stellt eine eigene Kategorie von Heuristiken dar (vgl. Forster 2013, S. 4). Sie umfasst Prozesse der Urteilsbildung, die darauf basieren, welche Informationen für eine Entscheidung kognitiv leicht abrufbar sind.

Simons begrenzte Rationalität zeichnet sich vor allem durch zwei Dinge aus: die Grenzen des menschlichen Geistes und die Anpassungsfähigkeit an die eigene Umwelt, in der die Person operiert. Die zweite Komponente ist nach seiner Ansicht die Entscheidende für Heuristiken: Heuristiken kommen dann zum Zuge, wenn das Verhalten an die Umweltstruktur angepasst wird. Dieses adaptive Verhalten produziert Heuristiken, die sich natürlich in der Umwelt bewegen. Im Gegensatz zum Ansatz 
4.5.2 Heuristische Entscheidungsregeln in der Personalauswahl

der begrenzten Rationalität berücksichtigen sie das Lernund Sozialisationsverhalten. Die Satisficing-Heuristik verlinkt eine begrenzte Anzahl von Hinweisreizen (cues) aus der Umwelt mit den vorhandenen Gedächtnisinhalten. Die Gruppenmitglieder suchen darin nach Informationen, um eine angemessene Entscheidung treffen zu können. Der Ansatz der fast and frugal heuristics arbeitet auf der Verhaltens- und Kognitionsebene mit der gleichen Basis. Fast and frugal heuristics suchen so lange nach einer angemessenen Entscheidungsalternative, bis diese ihren Entscheidungsregeln entspricht. Hierfür genügt sogar manchmal eine einzige Information. Insbesondere die Kombination von vielfältigen Informationen kompliziert eine Entscheidung, sodass der menschliche Geist automatisch dazu neigt, nicht überlastet zu werden und noch weitere Optionen zu simulieren oder Informationen zusammenzutragen. 
4.5.2 Heuristische Entscheidungsregeln in der Personalauswahl

\begin{tabular}{|c|c|c|c|}
\hline $\begin{array}{l}\text { Einstufung von } \\
\text { Heuristiken }\end{array}$ & Heuristik & Relevanz fur die Personalauswahl & Quelle \\
\hline $\begin{array}{l}\text { Verfügbarkeits- } \\
\text { heuristiken }\end{array}$ & $\begin{array}{l}\text { Verfügbarkeits- } \\
\text { heuristik }\end{array}$ & $\begin{array}{l}\text { Wenn Bewerberinformationen (oder anderes } \\
\text { auswahlrelevantes Wissen) leichter oder } \\
\text { schwerer verfügbar sind, wird die } \\
\text { Wahrscheinlichkeit des Eintretens über- oder } \\
\text { unterschātzt. Relevante Informationen werden } \\
\text { nicht wahrgenommen und ignoriert oder nicht } \\
\text { hinterfiragt. Bewerber/Bewerberinnen oder } \\
\text { Bewerberinformationen werden nicht } \\
\text { ausreichend berücksichtigt. }\end{array}$ & $\begin{array}{l}\text { Tversky und } \\
\text { Kahneman } \\
(1974)\end{array}$ \\
\hline $\begin{array}{l}\text { Ignarance-Based } \\
\text { Decision Making }\end{array}$ & $\begin{array}{l}\text { Rekognitions- } \\
\text { heuristik }\end{array}$ & $\begin{array}{l}\text { Die Wiedererkennbarkeit dient als einzige } \\
\text { Entscheidungsstrategie bei vergleichenden } \\
\text { Urteilen. Zum Beispiel Wiedererkennen von } \\
\text { Bewerbereigenschaften, wie Qualiikation, } \\
\text { Erfahrung und Persōnlichkeit }\end{array}$ & $\begin{array}{l}\text { Gigerenzer } \\
\text { et al. (1999), } \\
\text { Reimer/Katsik } \\
\text { opoulos } \\
\text { (2004): für } \\
\text { Gruppen }\end{array}$ \\
\hline $\begin{array}{l}\text { One-Reason } \\
\text { Decision Making }\end{array}$ & $\begin{array}{l}\text { Take-the-best } \\
\text { Heuristik }\end{array}$ & $\begin{array}{l}\text { Diese Heuristk dient z.B. dazu, sich zwischen } \\
\text { Bewerbem/Bewerberinnen } \\
\text { denjenigen/diejenige zu entscheiden, bei } \\
\text { dem/der ein gewāhltes Kriterium z.B. "viel } \\
\text { Berufserfahrung“ die hōchste Ausprāgung hat. }\end{array}$ & $\begin{array}{l}\text { Gigerenzer et } \\
\text { al. (1999a) }\end{array}$ \\
\hline
\end{tabular}

Abbildung 14: Kategorisierung von Heuristiken (in Anlehnung an Gigerenzer und Todd (1999b, S. 359).

Die in Abbildung 14 eingeordneten und kurz vorgestellten Heuristiken sollen im folgenden Kapitel konkretisiert und mit der Personalauswahl verknüpft werden. Die Auswahl dieser Heuristiken wurde anhand einer möglichen Relevanz für die Personalauswahl festgelegt. 


\subsubsection{Verfügbarkeitsheuristik}

Der Prozess des Problemlösens ist alles andere als trivial, und es werden vielfältige Heuristiken angewandt, die es Individuen oder Gruppen erlauben sollen, trotz begrenzter Ressourcen (Zeit, Energie etc.) eine adäquate Entscheidung zu treffen. Um Zeit und Ressourcen zu sparen, kommen Organisationen oft nicht umhin, Entscheidungswege zu wählen, die nicht dem Detailierungsgrad von Personalauswahlprozessen entsprechen, der dem/der Bewerber/-in und den Folgen der Stellenbesetzung Respekt zollen würde. Wie bereits in Kapitel 3.3.2 dargestellt, können auch Gruppen Heuristiken nutzen, um ohne großen Aufwand machbare Entscheidungen zu treffen. Ihre Entscheidungsfindung unterliegt der begrenzten Rationalität, und sie wenden ebenfalls abgekürzte Entscheidungswege an. Die Gruppe kann über gemeinsames, d.h. geteiltes Wissen verfügen, welches sie erworben hat, wenn die Mitglieder bereits zuvor Kenntnisse in der Personalauswahl erwerben konnten (siehe Kapitel 3.3.3.3). Sie erhalten dadurch eine Expertise, die auf der Erfahrung der einzelnen Mitglieder sowie auf der Organisations- und Gruppenerfahrung beruht. Die kollektive Erfahrung von Gruppen und Organisationen 
4.5.2.1 Verfügbarkeitsheuristik

aus der Vergangenheit kann richtungsweisend für deren aktuelles Entscheidungsverhalten sein. Sie kann dazu führen, dass in einer Entscheidungssituation Entscheidungsregeln generiert werden, die sich aus der Dynamik der Gruppengemeinschaft ergeben, z.B., indem sie sich an eine Entscheidung vor einem Jahr erinnern, in der sich die Gruppe für die Einstellung eines Einkäufers mit viel Branchen- und Berufserfahrung ausgesprochen hat. Durch diese Entscheidung konnten hohe Einsparungen erreicht werden. Wenn die Mitglieder nun vor einer ähnlichen Einstellungsfrage stehen, kann auf diese Erfahrung zurückgegriffen werden. Die kollektive Erfahrung führt zu einem Handlungswissen der Gruppe, das sich aus den Lern- und Sozialisationsprozessen ergeben kann (sog. „Schwarmwissen“). Die Heuristik basiert dann auf dem Handlungswissen der Gruppe und kann auf einen neuen Einstellungsfall angewandt werden. Der „Fall“ aus der Vergangenheit wird übertragen, ohne alle einzelnen Einstellungskriterien oder Bewerberinformationen geprüft zu haben.

Auch die Verfügbarkeitsheuristik greift auf Wissen der Gruppenmitglieder zurück und speist sich aus unvollständigen Informationen. Der Prozess der Einschätzung 
von Häufigkeiten, sich an Beispielfälle aus der Vergangenheit leicht zu erinnern, basiert bei der Gruppe auf dem Zusammenwirken und der Kommunikation der Mitglieder: Besteht eine Gruppe schon länger, ist es denkbar, dass jeder/jede zum einen auf die Erfahrung der letzten Entscheidung zurückgreifen kann und zum anderen die in der Zwischenzeit aufgetretenen Fälle als Beispiele zur Entscheidungsvorbereitung abrufen kann. Die verfügbare Heuristik basiert auf gemeinsamen Erkenntnissen und Erfahrungen. Diese gemeinsam verfügbaren Bilder können leichter aus dem Gedächtnis abgerufen werden, weil sie auf persönlicher Erfahrung beruhen (vgl. Kahneman 2011, S. 165). Durch die individuelle „Betroffenheit" steigt die Verbindung zu dem Ereignis oder der Aufgabe und ermöglicht das rasche Aufrufen von Beispielfällen. Die Häufigkeit, mit der vergangenes Handlungswissen abgerufen wird (viele Beispiele aus dem Gedächtnis), gibt den Gruppenmitgliedern das Gefühl, dass diese Beispielfälle allgegenwärtig sind (vgl. Riesenhuber 2006, S. 87): Wenn man selbst von Fahrraddiebstahl betroffen war, hat man das Gefühl, dass die Wahrscheinlichkeit von Fahrraddiebstählen im Umkreis statistisch gesehen höher ist, als dies tatsächlich der Fall ist. 
Diese Verfügbarkeitsheuristik führt dazu, dass Wahrscheinlichkeiten über- oder unterschätzt werden. Wenn einem ein Ereignis schneller einfällt, kommt man zu dem Schluss, dass es häufiger auftritt, bzw. wenn man sich das Ereignis leicht vorstellen kann, dann wird es häufiger vorkommen. Wahrscheinlichkeiten werden dabei häufig verdrängt, überschätzt oder ignoriert (vgl. Kahneman 2011, S. 181).

Hervorstechende (interne oder externe) Ereignisse, die in der Vergangenheit der Organisation, der Gruppe oder Umwelt besonders präsent waren, werden von den Gruppenmitgliedern über- oder unterschätzt. Wie in dem vorgenannten Einstellungsbeispiel des Einkäufers bezieht sich das "Schwarmwissen" bei Personalentscheidungen auf vergangene Erfahrungen, die zu einem Handlungswissen werden und auf organisationalem oder umweltbezogenem Erfahrungswissen beruhen.

Umweltbezogenes Erfahrungswissen für die Personalentscheidung der Gruppe bezieht sich auf alle Themen, die die Gruppe tangieren. Hierzu gehören z.B. Stakeholder-Informationen oder Medienberichte. Zum Beispiel die (stark medienwirksame) Rechtsprechung der Emily- 
Entscheidung des $\mathrm{BAG},{ }^{41}$ bei der eine Kassiererin mit langer Betriebszugehörigkeit zwei Kassenbons im Wert von 1,30 Euro entwendete und daraufhin vom Arbeitgeber gekündigt wurde. Dieser Fall wurde intensiv unter Personalverantwortlichen diskutiert. Ein weiterer prägnanter Fall ist die AGG-Frage der Minderheitendiskriminierung $^{42}$ in der Personalauswahl. Für Personalverantwortliche ist die Gefahr, in Einstellungsprozessen Minderheiten zu diskriminieren, durch die Einführung des AGG präsenter geworden, weil diese durch den Gesetzgeber mit Sanktionen verbunden wurden. Personalverantwortliche können sodann die Wahrscheinlichkeit einer Diskriminierungsanzeige überschätzen. Die Anwendenden der Verfügbarkeitsheuristik nehmen diese Ereignisse als höchst wahrscheinlich oder typisch wahr, als Indikatoren für ihre eigene Situationsbewertung bei der Personalauswahl. Dies kann zur Folge haben, dass sie sehr

\footnotetext{
${ }^{41}$ Das Bundesarbeitsgericht (BAG) in Erfurt erklärte die verhaltensbedingte Kündigung einer Kassiererin am 10. Juni 2010 für unverhältnismäßig und damit für unwirksam (Az. 2 AZR 541/09).

42 Das Allgemeine Gleichbehandlungsgesetz (AGG) nennt Diskriminierungsmerkmale, wie Behinderung, Religion, Alter, Geschlecht etc. Die Rechtsfolgen unerlaubter Ungleichbehandlungen sind Beschwerderechte, Entschädigungsansprüche, Leistungsverweigerungsrecht etc.
} 
viel bewusster mit Minderheiten umgehen, weil sie sich vor Diskriminierungsanzeigen fürchten.

Beispiel: Personaler, die gebeten werden, zu schätzen, wie oft Bewerber/-innen eine Diskriminierungsanzeige vor Gericht erheben, weil sie sich auf das AGG berufen (wenn z.B. eine Frau bei der Einstellung trotz besserer Qualifikation abgelehnt wurde), überlegen sich nach dem Modell der Verfügbarkeit, wie oft sie schon davon gehört haben, dass es zu einer Diskriminierungsanzeige gekommen ist. Wenn sie sich an viele Berichte erinnern, die AGG-Sachverhalte erörtern oder zur Anzeige gekommen sind, können sie darauf schließen, dass es häufiger vorkommen könnte, als dies vielleicht tatsächlich der Fall ist. Dieses umweltbezogene Wissen übertragen sie auf ihre eigene Entscheidung.

Umweltbezogenes, organisationseigenes oder individuelles Wissen fließt in die Entscheidungen der Personalauswahlgruppe ein. Mit diesem abrufbaren Wissen im Hinterkopf bewegt sich die Gruppe in Richtung Entscheidung. Riesenhuber (2006, S. 88) bezeichnet diesen Schritt als „vorgewärmte Gedächtnisstrukturen“. Die verfügbaren Informationen der Gedächtnisstrukturen leiten 
4.5.2.1 Verfügbarkeitsheuristik

die Gruppe und erhöhen die Aufmerksamkeit für den Prozess der Einschätzung. Leicht verfügbare Erinnerungen aus gut zugänglichen Quellen können so auch von mehreren angewendet werden (vgl. Auer-Rizzi 1998, S. 132).

Die Aspekte der Verfügbarkeitsheuristik gelten deshalb nicht nur für Individuen. Die Gruppe unterliegt genauso dem Irrtum, dass bereits Gehörtes und leicht Erinnerbares auch für sie allgegenwärtig sei. Für die Personalauswahl kann das Folgendes bedeuten: Eine hohe Verfügbarkeit führt zu einer höheren Aufmerksamkeit der Gruppe in Bezug auf Einzelinformationen, wie die vorherigen Personalbeispiele aus der Rechtsprechung oder den Medien zeigen. Diese Informationen werden für den eigenen Fall abgeleitet und im Sinne der Verfügbarkeitsheuristik deutlich über- oder unterschätzt. Die mediale Diskussion um Diskriminierungen bei Einstellungen kann zum Beispiel in der Gruppe zu einer starken Verfügbarkeit dieser Informationen führen. Es wird dann die Wahrscheinlichkeit unter- oder überschätzt, dass ein solches Szenario in der eigenen Organisation auch auftreten kann. Die verfügbaren Informationen können damit sogar in der Diskussion präsenter sein, wodurch die Organisa- 
4.5.2.2 Rekognitionsheuristik

tionsmitglieder überproportional sensibilisiert werden.

Jedoch wird in der Auswahlsituation vernachlässigt, dass das Eintreten eines Ereignisses in der Organisation weit weniger wahrscheinlich ist, als die Organisationsmitglieder vermuten. Es handelt sich oft um Informationen, die leichter erinnert werden, wenn sie häufig erlebt wurden, weil sie vertraut bzw. sehr lebendig sind, anschaulich präsentiert worden sind oder erst kurze Zeit zurückliegen (vgl. Tversky/Kahneman 1974, S. 1127).

\subsubsection{Rekognitionsheuristik}

Eine weitere Heuristik aus der adaptiven Toolbox von Gigerenzer (siehe Kapitel 4.5) ist die Rekognitionsheuristik. Sie beruht auf der einfachen Unterscheidung, ob ein Objekt wiedererkannt wird oder nicht. Diese Heuristik hilft in Auswahlsituationen, in denen nur sehr wenig Wissen vorhanden ist.

Die Rekognitionsheuristik ist eine Entscheidungsstrategie für vergleichende Urteile, bei denen es um das Erkennen bzw. Nicht-Erkennen eines Objekts geht. Die Entscheidung basiert ausschließlich auf dieser Wiedererkennungsinformation (Rekognition). Weitere Informationen 
4.5.2.2 Rekognitionsheuristik

werden ignoriert (ignorance-based decisionmaking). Im Gegensatz zur Verfügbarkeitsheuristik, die sich auf recall (die Fähigkeit, Gedächtnisinhalte abzurufen) bezieht, ist recognition das Wiedererkennen von Objekten, um daraus zu schließen, dass das wiedererkannte Objekt den höheren Wert auf dem Kriterium hat (vgl. Gigerenzer/Gaissmaier 2006, S. 9 f.). Tatsächlich hilft diese Heuristik aber nur den wenig Wissenden, wenn also Personen das Gefühl haben, etwas wiederzuerkennen. Das heißt, sie glaubten lediglich, davon gehört zu haben (vgl. Gigerenzer 2007, S. 26).

Um zu einer Entscheidung zu gelangen, reduziert das Individuum zunächst seine Informationen bereits von sich aus, um der Komplexität seiner Umwelt Rechnung zu tragen. Das ist das Wesen von Heuristiken. Genauso verhält es sich auch bei der Rekognitionsheuristik. Aber auch in der Gruppe?

Auch im Kollektiv der Gruppe ist eine Informationsreduzierung möglich, da sich vielfach erst im Laufe des Entscheidungsprozesses noch weitere Informationen oder Auswertungen ergeben, die der Einzelne noch nicht vollumfassend wissen konnte (z.B. durch ungeteiltes Wissen - siehe Hidden Profile). Soziale Phänomene der Organi- 
4.5.2.2 Rekognitionsheuristik

sationsmitglieder können dazu führen, dass sich die Gruppenmeinung z.B. von einer initialen Ablehnung am Anfang (disagreement) zu einer Zustimmung bewegt (vgl. Baron et al. 1992, S. 93). Dies geschieht in den meisten Fällen durch Kommunikation. Hierzu gehört die ganze Bandbreite sozialer Phänomene, wie Hierarchien, Durchsetzung, Machtverhältnisse etc.

Auch die Fragestellungen der Gruppe können mithilfe von Rekognitionsheuristiken vereinfacht abgearbeitet werden, indem Informationen bei vergleichenden Entscheidungen von den Gruppenmitgliedern wiedererkannt werden. Dennoch sind Untersuchungen zu heuristischen Entscheidungen von Gruppen selten. In einer Studie zur Rekognitionsheuristik bei Gruppenentscheidungen von Reimer et al. (2004) wurde das gleiche Untersuchungsdesign wie bei Gigerenzer und Todd (1999a) verwendet, um eine Anwendbarkeit zu prüfen: 
Einer Gruppe von drei Personen wurde die dichotome Frage ${ }^{43}$ gestellt: „Welche Stadt hat mehr Einwohner? San Antonio oder San Diego?"

Von den US-Amerikaner/-innen wählten 62\% San Diego als richtige Alternative aus, während die Antwort von $100 \%$ der Deutschen richtig angegeben wurde. Dafür könnte die Rekognitionsheuristik verantwortlich sein: Wenn man von zwei Städten nur eine kennt, nimmt man diese als die größere an. Die Gruppenmitglieder verließen sich intuitiv auf die richtige Entscheidung, trotz fehlender Informationen. Dieser Effekt tritt auch in Gruppen auf. Auer-Rizzi (1998, S. 159) führt an, dass Gruppen ebenso anfällig für Entscheidungsverzerrungen sind wie Individuen, ${ }^{44}$ weil die sozialen Normen, „etliche Eigenhei-

${ }^{43}$ Zunächst war die Rekognitionsheuristik auch auf den Vergleich von zwei Objekten beschränkt, jedoch wurde sie später erweitert und mehr Auswahlobjekte wurden validiert (vgl. Marewksi et al. 2010, S. 387 f.).

${ }^{44}$ Eine weitere Entscheidungsregel auf kollektiver Ebene ist die Ankerheuristik. Tversky und Kahneman (1974, S. 1128 f.) befragten Probanden: „Wie hoch ist der Anteil afrikanischer Staaten in der Uno? Ist der Anteil höher oder niedriger als 65\%?" Und: „Ist der Anteil höher oder niedriger als 10\%?" Bereits der Anker (65 oder 10\%) in der Fragestellung führt zu einem geänderten Antwortverhalten. Der Ankerheuristik können sich auch Gruppen nicht verschließen (vgl. Neumer 2009, S. 31), d.h., auch sie lassen die Ankerheuristik in ihre gemeinsame Entscheidungsfindung fließen. 
4.5.2.2 Rekognitionsheuristik

ten des Gruppenprozesses“ und Effekte zu einer Verzerrung führen können.

Beispiel: In Gruppendiskussionen werden oft Argumente ausgetauscht, die schon allen bekannt, und weniger solche Argumente, die nur einzelnen Mitgliedern bekannt sind. Oder die soziale Dynamik führt zu einer Diffusion von Verantwortung, was zur Folge hat, dass die Mitglieder sich gegenseitig darin bestärken, sozialen Normen zu trotzen.

In ihrer Studie untersuchten Reimer et al. (2007) die Frage, ob eine Gruppe, die weniger Objekte wiedererkennt, bessere Urteile fällen kann als Individuen. Ihre Ergebnisse beschreiben die Repräsentativitätsheuristik nicht als Verzerrung. Im Gegenteil, sie stellen sogar eine bessere Entscheidung in Aussicht als ohne Heurististik. Statistische Wahrscheinlichkeiten können auch von der Gruppe ignoriert werden, da sie Wahrscheinlichkeiten von Ereignissen, die hervorstechen, überschätzt (vgl. Auer-Rizzi 1998, S. 131). So könnte es sich auch mit der Rekognitionsheuristik bei der Personalauswahl verhalten. In einer natürlichen Umgebung wie der Personalauswahlsituation sind die Erinnerungen Einflusskriterien der Gruppenmitglieder. 
Die Take-the-best-Heuristik (siehe Abbildung 14) stellt einen Teil der Rekognitionsheuristik stellt dar, da sie anwendbar ist, wenn eines von zwei zu vergleichenden Objekten wiedererkannt wird. Die Take-the-best-Heuristik beschränkt die Informationsverarbeitung, indem sie verfügbare Informationen systematisch ignoriert (vgl. Reimer et al. 2007, S. 9).

Die heuristische Entscheidungsregel „Wir stellen nur die Besten ein“ kann eine Take-the-best-Heuristik (,Ignoring cues to make better predictions") darstellen (vgl. Gigerenzer/Brighton 2009, S. 113). Bei der Personalauswahl werden Informationen über die Stellenbewerber/-innen miteinander verglichen. Die verschiedenen Entscheidungsobjekte der Personalauswahl sind die Bewerber/innen mit ihren zahlreichen, höchst individuellen Ausprägungen von Qualifikation, Erfahrung und Persönlichkeit.

Diese Heuristik kann dazu dienen, sich zwischen Bewerbern/-innen für denjenigen/diejenige zu entscheiden, bei dem/der das gewählte Kriterium, z.B. „viel Berufserfahrung", die höchste Ausprägung hat (vgl. Gigerenzer/Marewski 2013, S. 233). Gruppenmitglieder würden Informationen mit der ökologisch rational höchsten Validi- 
tät auf dem Lösungskriterium wählen (vgl. Lyding 2010, S. 11), d.h., z.B. einfach die Berufsjahre als Kriterium für die Berufserfahrung nehmen. Welche Alternativen die höhere Ausprägung auf einem Kriterium vorweisen, wird von den Gruppenmitgliedern festgelegt. Untersuchungen (u.a. von Reimer et al. 2007, S. 16 f.; Gigerenzer/Goldstein 1996, S. 650 f.) haben ergeben, dass die Take-the-best-Heuristik in vielen Informationsumgebungen zu einer sehr hohen Trefferquote führen kann. Die Take-the-best-Heuristik ist für ein Aufgabenumfeld mit vielen Informationen von Vorteil, weil sie nur über einen geringen Anteil am vorhandenen Wissen verfügt (vgl. Reimer et. al. 2007, S. 16). Im Falle der Personalauswahl müssen danach nicht alle Bewerberinformationen für die Entscheidung zurate gezogen werden, sondern lediglich ausgewählte. Beispiel: Wenn der/die Bewerber/-in seine Hobbys nicht angegeben hat, ist diese Information unter Umständen im Bewerbergesamtbild vernachlässigbar. Auch Gigerenzer und Marewski (2013, S. 238) schlagen deshalb die Take-the-best-Heuristik (sowie Tallying ${ }^{45}$ ) als

${ }^{45}$ Beim Tallying werden die Argumente bis zur Erreichung eines Grenzwertes gesammelt. Die Gruppe addiert also so lange cues, bis 
4.5.2.2 Rekognitionsheuristik

heuristische Entscheidungshilfe vor, die sich gut zur diagnostischen Vorhersage bei der Auswahl von Personal eignen könnte. Sie betonen aber auch, dass heuristische Methoden im Gegensatz zu personaldiagnostischen Methoden, wie Interviews, Persönlichkeitsinventare, Intelligenztests, Verhaltensbeobachtung oder AssessmentCenter, stehen. In der vorliegenden Arbeit sollen Heuristiken ergänzenden Charakter haben und die Personalauswahl unterstützen und weder als alleinige Methode taugen noch mehrstufige Auswahlverfahren in Organisationen untergraben.

Die Rekognitionsheuristik kann Aufschluss über die Informationsverarbeitungsstrategie von Gruppenmitgliedern geben. Außerdem räumt sie mit dem Fehlschluss auf, dass gute Entscheidungen einen möglichst vorständigen Informationsaustausch unter den Gruppenmitgliedern voraussetzen (vgl. Reimer et al. 2007, S. 16).

Eine Toolbox der Personalauswahl müsste einen größeren Umfang haben als an dieser Stelle behandelt. Trotzdem ist es für den Leitgedanken „Personalentscheidun-

eine bestimmte Schwelle erreicht ist (vgl. Gigerenzer/Gaissmaier 2006, S. 15). 
4.6 Fazit: Bewertung der heuristischen Personalauswahl gen werden in Gruppen getroffen" entscheidend, dass Heuristiken dabei eine maßgebliche Rolle spielen. Die ausgewählten Heuristiken könnten Teil einer solchen Untersuchung „Toolbox der Personalauswahl“ als zentrales Thema werden. Im folgenden Kapitel soll eine Bewertung von heuristischen Gruppenentscheidungen die Frage beantworten, ob Heuristiken bei Personalentscheidungen Fehlentscheidungen sind.

\subsection{Fazit: Bewertung der heuristischen Personalauswahl}

In Kapitel 4.5.1 konnte dargestellt werden, dass es oft keine kausale Verbindung zwischen der Personalauswahl und dem Erfolg eines/einer Bewerbers/-in gibt. Damit stellt sich die Frage, wie die heuristische Personalauswahl abschließend bewertet werden kann. Hierzu sollen drei Aspekte zusammenfassend dargestellt werden:

1. Zusammenhang zwischen Entscheidung und Bewerberperformance

2. Komplexität und fehlendes (Normen-)Gerüst der Gruppenauswahl 
4.6 Fazit: Bewertung der heuristischen Personalauswahl

3. Heuristische Informationsverarbeitung

\section{Zusammenhang zwischen Entscheidung und Be- werberperformance}

Wenn die Performance eines/einer Bewerbers/-in in der Organisation nicht den gewünschten Erfolg hat, muss dies nicht an der Personalauswahlentscheidung der Organisation liegen, sondern kann andere Gründe haben, wie etwa eine schlechte Integration ins Team, eine schlechte Einarbeitung oder einen fehlenden Zugang zu Informationen. Umgekehrt können auch "gut ausgewählte" Bewerber/-innen scheitern. Dies geschieht dann jedoch nicht aufgrund fehlender Qualifikation oder Kompetenz, sondern aufgrund von Organisationsbedingungen. Fehleinschätzungen und Fehlurteile bei der Personalentscheidung werden meist als Risiken kategorisiert (u.a. Kanning 2004, S. 58). Doch eine unzureichende Prüfung von stellenrelevanten Bewerberinformationen oder Entscheidungsfehlern (wie Halo-Effekt) im Prozess führt noch zu keinem Fehlurteil. Selbst wenn (bewerberspezifische) Informationen außer Acht gelassen, fehlgedeutet o.ä. werden, kann der Entscheidungsprozess im Allgemeinen zwar als defizitär betrachtet werden (siehe hierzu 
4.6 Fazit: Bewertung der heuristischen Personalauswahl auch Kapitel 4.5.1), aber eine kausale Verlinkung mit dem Berufserfolg gelingt auch aus dem Grund nicht, weil stellenrelevante Fertigkeiten, Fähigkeiten und Kenntnisse erworben werden können. Auch wenn der Berufsstart in der Organisation „schleppender“ ausfällt als geplant und nicht den Erwartungen der Organisation gerecht wird (Performance), überwiegt die Methodenkompetenz, sich Wissen anzueignen. Die defekte Einschätzung kann durch heuristische Elemente in der Entscheidung entstanden sein, muss aber nicht kausal sein.

Entscheidungen, die auf heuristischen Urteilen basieren, geben zwar kein rationales Urteil ab, aber sie unterstützen die Urteilsfindung als Entscheidungshilfe. Dies gelingt insbesondere dann, wenn die heuristische Entscheidung in Verbindung mit den analytischen Elementen der Personaldiagnostik gebracht wird. Mit dieser Sichtweise folgt die Arbeit nicht der Forderung (z.B. nach mehr Standardisierung durch DIN-Normen oder strukturierte Interviews), den Einfluss von Intuition und heuristischen Entscheidungshilfen im Rahmen der diagnostischen Personalentscheidung zu minimieren. 
4.6 Fazit: Bewertung der heuristischen Personalauswahl

\section{Komplexität und fehlendes (Normen-)Gerüst bei der Gruppenauswahl}

Die Gruppe soll die Frage beantworten, welcher/welche Bewerber/-in eingestellt wird bzw. wer zurückgewiesen wird. Dazu müssen die Ergebnisse der Personaldiagnose von den Gruppenmitgliedern ausgewertet und bewertet werden, um abschließend eine Entscheidung als Gesamturteil zu fällen. Je standardisierter das Verfahren ist, desto weniger Spielraum erhält die Gruppe in der Entscheidungsfindung (vgl. Kanning 2004, S. 268). Doch bei der Personalauswahl genügen Vorgaben der Organisation nicht, und auch Mittelwerte, Einstellungsbeispiele oder Normen bieten wenig Orientierungshilfe für die Gruppe: Ob ein/eine Bewerber/-in intelligenter als der Durchschnitt der Gesamtbevölkerung ist, ist für die Besetzung einer Stelle häufig von untergeordneter Relevanz. Meist geht es um die Frage, ob der/die Bewerber/-in über eine angemessene Intelligenz zur Bewältigung der Aufgabe verfügt (a.a.O.). Für die Personalentscheidung gibt es also wenig "Greifbares" für eine evaluierte Entscheidung. Die Gedächtnisinhalte der Teilnehmenden werden für die Auswahl nach ähnlichen Ereignissen durchsucht, die sich aber auf allgemeine Entscheidungsregeln beziehen und 
4.6 Fazit: Bewertung der heuristischen Personalauswahl weniger auf Normen. Gigerenzer (1996, S. 192 f.) diskutiert diesen fehlenden Normbezug unter dem Stichwort "narrow norms" und geht auch der Frage nach, ob es, wenn Normen fehlen, dann überhaupt richtige oder falsche Entscheidungen geben kann. Diese Sichtweise teilt auch Riesenhuber (2006, S. 86): Als fehlerhaft können Ergebnisse nur dann interpretiert werden, wenn das richtige Ergebnis überhaupt postuliert wurde.

Komplexität bezieht sich auf die beurteilenden Reize und die kognitiven Strukturen der an dem kollektiven Entscheidungsprozess beteiligten Personen (vgl. Ahrens 1976 S. 35). Die Komplexität der Personalentscheidung bezieht sich auf die Unübersichtlichkeit der Organisation, die Bandbreite an Berufen innerhalb und außerhalb der Organisation sowie die zahlreichen Umweltbedingungen. Um trotzdem hinreichende Bedingungen in die Entscheidungsfindung der Gruppe zu integrieren, können Standards der Personalauswahl der Gruppe die Unsicherheit in ihrer Auswahlentscheidung nehmen. Aber Standards berücksichtigen weder alle Einstellungsaspekte noch können sie der Komplexität naturalistischer Entscheidungsverfahren Herr werden. Nicht selten muss eine Gruppe mehr als fünf Kriterien in ihrer Auswahl zur Ent- 
4.6 Fazit: Bewertung der heuristischen Personalauswahl scheidungsfindung einbeziehen. Kanning (2004, S. 269) gibt folgendes Beispiel zur Auswahl eines Pressesprechers: Der/die Bewerber/-in soll verbale Kompetenzen, eine gute Auffassungsgabe, soziales Verhalten, organisatorische Fähigkeiten, Stressresistenz, fachliches Wissen u.Ä. mitbringen. Allein die Kombinationsvielfalt der Ausprägungen dieser Einstellungskriterien ist hoch. Daraus ergibt sich, dass heuristische Entscheidungen für die Gruppe unumgänglich sind, weil die kognitiven Kapazitäten der Mitglieder zu begrenzt sind, um die vielfältigen Ausprägungen sowie die Komplexität auf rationalanalytischem Wege zu filtern. Ein Teil der Komplexität der Aufgabenstellung „Personalentscheidung“ wird für die Gruppe deshalb durch Heuristiken reduziert.

\section{Heuristische Informationsverarbeitung in der Per- sonalauswahl}

Die Personalentscheidung stützt sich oft auf Daten, die nach heuristischen Regeln verarbeitet werden. Beispielsweise wenn die Einschätzung des/der Bewerbers/in nach einem Interview nicht zeitnah oder später erfolgt, z.B. die Informationen aus dem Bewerbergespräch nicht mehr präsent sind, weil es zu lange zurückliegt und des- 
4.6 Fazit: Bewertung der heuristischen Personalauswahl halb nicht gut im Gedächtnis „verblieben“ ist. Die Kontur der Bewerberinformationen wird verwässert, was zu einer heuristischen Informationsverarbeitung führt. Die zeitliche Nähe von Bewerberinformationen vereinfacht die Personalauswahl der Gruppe. Informationen müssen nicht erneut ausgetauscht werden oder werden nicht im Laufe der Zeit verzerrt. Der Vergleich mit anderen Bewerbern/innen ist für die Gruppe präsenter, wenn die Bewerberinformationen allen deutlich im Gedächtnis verbleiben und ihnen dadurch kognitive Last abgenommen wird. In einer Studie von Stephan und Westhoff (2002) mit mehr als 100 Unternehmen gaben 25\% der Gesprächsteilnehmenden an, die Gesprächsauswertung erst nach mehreren Tagen vorzunehmen, $78 \%$ taten dies erst nach mehreren Gesprächen (S. 11 f.). Inhaltlich basiert diese Auswertung auf eher allgemeinen Kriterien, jedoch zu 75\% auf dem Anforderungsprofil. Diese Angabe würde bedeuten, dass lediglich $25 \%$ sich auf andere als die objektiven Kriterien des Anforderungsprofis stützen würden. Da die Daten aber auf einer Selbsteinschätzung der Entscheidenden beruhen, ist wohl eher der Wunsch der Vater des Gedankens. Andere Studien weisen weit höhere Werte des subjektiven Einflusses bei der Entscheidung aus: 
4.6 Fazit: Bewertung der heuristischen Personalauswahl

Dies ist z.B. dann der Fall, wenn den Gruppenmitgliedern die Anforderungen nicht oder nur vage bekannt sind, sodass die Personalentscheidung eher auf intuitiven Kriterien beruht und z.B. auf Basis von Sympathie oder Antipathie gefällt wird (vgl. Weuster 2012a, S. 38).

Gerade innerhalb der ersten Stufen eines mehrstufigen Einstellungsprozesses sind die Informationen der Gruppe oft weder vollständig noch verfügbar, da das Einstellungsverfahren noch weitere Stufen durchläuft und deshalb schlicht noch nicht abgeschlossen ist. Trotzdem werden bereits in einer frühen Phase die vorhandenen Informationen zu einer Vorauswahl der Bewerber/-innen genutzt (vgl. Cascio/Reynolds 2011, S. 59). Diese Vorauswahl kann einer Vorentscheidung gleichkommen, die noch nicht zu einer Auswahlentscheidung geführt hat, aber eine Vorbereitung mit meinungsbildendem Charakter repräsentieren kann. Um die erste Fülle der Bewerbungen und die damit einhergehenden Informationen zu strukturieren, haben die Informationen den Charakter einer Filtrierung. Wobei bereits einige Informationen über die Bewerber/-innen zu einem Ausschluss führen, d.h., es wird eine Preselektion durchgeführt (Cut-off-Effekt). 
4.6 Fazit: Bewertung der heuristischen Personalauswahl

Der heuristische Entscheidungsprozess kann jedoch mithilfe der Formulierung expliziter Entscheidungsregeln und der Definition von cut-offs in pragmatische Entscheidungsregeln überführt werden (vgl. Koppers 2013, S. 38). Die negative Selektion von cut-offs schränkt den Auswahlradius der Gruppe ein, trifft aber noch keine finale Auslese. Unterstrichen wird dieser selektive Charakter der Informationsverarbeitung durch die Dynamik der Gruppe. Da in jeder Einstellungsstufe eine Entscheidung zu jedem/jeder Bewerber/-in erwartet werden kann, tritt die Gruppe bereits zu Beginn zusammen und entscheidet darüber, ob der/die Bewerber/-in eine Stufe „weiterkommt" oder nicht. Weil die Personalentscheidung von den einzelnen Teilnehmenden oft in einer frühen Phase des Einstellungsinterviews getroffen wird, ist das weitere Verhalten der Interviewer/-innen im Gespräch gesteuert, und die Endauswahl wird dadurch beeinflusst (vgl. Weuster 2012b, S. 52).

Die drei Aspekte (1. Zusammenhang zwischen Entscheidung und Bewerberperformance, 2. Komplexität und fehlendes (Normen-)Gerüst der Gruppenauswahl und 3. heuristische Informationsverarbeitung) sind Kriterien für 
4.6.1 Heuristiken von Experten/-innen

eine heuristisch geprägte Personalauswahl.

Fehlende Vergleichsnormen und die abgekürzte Informationsverarbeitung der Gruppe zeichnen das Bild einer unumgänglich heuristischen Personalauswahl. Sie können gar nicht ausgeschlossen werden, und die Personalauswahl kann nicht ohne sie getroffen werden, bzw. sie wird nicht rational getroffen. Lediglich die defizitären Konsequenzen von Heuristiken können minimiert werden. Diese Bewertungskritierien stellen eine wichtige Grundlage für weitere Betrachtungen dar, die den Ansprüchen einer angemessen Organisationsentscheidung genügen soll. Hierbei muss auch betrachtet werden, ob vielleicht Experten/-innen besser in der Lage sind, professionell mit heuristischen Entscheidungen umzugehen.

\subsubsection{Heuristiken von Experten/-innen}

Es wird gemeinhin davon ausgegangen, dass Experten/innen Informationen und Hinweisreize auf komplexe Weise zu kombinieren wissen und daraus eine kompetente Entscheidung ableiten. Doch fehlende Transparenz und Nachvollziehbarkeit bei Personalentscheidungen lassen 
4.6.1 Heuristiken von Experten/-innen

nicht nur an der Einstellungsentscheidung selbst, sondern auch an der Kompetenz von Personalentscheidern/innen zweifeln, die sich ihrer guten Menschenkenntnis rühmen (vgl. Kanning 2004, S. 58). Die Verbindung von Expertentum und Heuristiken ist deshalb ein wichtiger Aspekt für die Begründung, warum Heuristiken in der Personalauswahl eine zentrale Rolle spielen. Deshalb stellt sich im Folgenden die Frage, wie Heuristiken von Experten/-innen im Bereich der Personalauswahl dargestellt werden können, und vor allem, ob Heuristiken und Expertentum Gegensätze darstellen, wonach die einen das andere ausschließt.

Experte/-in zu sein, bedeutet für viele, auf in einem Gebiet versiert zu sein. Moser et al. (2000, S. 441) definieren Expertentum folgendermaßen. Die Regeln, die Experten/-innen heranziehen, können nicht generalisiert werden, ihre Heranziehung hängt vielmehr von Rekognitions- und Identitätsprozessen ab. Experte/-in zu sein, bedeutet also nicht nur, über ausreichend Wissen zu verfügen, betroffen oder involviert zu sein, sondern auch, auf Regeln für solche Situation zurückgreifen zu können, die sich aber von Experte/-in zu Experte/-in unterschei- 
4.6.1 Heuristiken von Experten/-innen

den. Umfangreiches Erfahrungswissen ermöglicht es den Experten/-innen, abgekürzt zu einer Auswahl zu gelangen. Das Besondere an dem/der Experten/-in ist, dass er/sie bestimmte Regeln erlernt hat, die man in bestimmten Situationen anwenden kann (vgl. Moser et al. 2000, S. 441). Er/sie wendet Entscheidungsregeln an, die das Ergebnis komplexer Lernprozesse sind und durch assoziative Mechanismen geprägt wurden (u.a. Lieberman 2000; zit. n. Koppers 2013, S. 2).

Das Anwenden von Heuristiken in der Personalauswahl kann sich auch darauf gründen, dass sich Experten/innen ihren Erfahrungsschatz zunutze machen, den sie über die Jahre in der Personalauswahl gesammelt haben und daraus Schemata klarer Entscheidungsregeln für sich abgeleitet haben. Die Entscheidung beruht also auf subjektiven Kriterien, die sie anhand von Hypothesen validiert und anhand von abgeleiteten Entscheidungsregeln für sich objektiviert haben. Ein subjektives Bedeutungsschema in der Auswahl muss aber nicht unbedingt ein Hindernis darstellen, sondern ermöglicht es Entscheidern/-innen, handlungsfähig zu bleiben und aufgrund von vorhandenem „Stamm-Kapital“ des Wissens und aufgrund ihrer Erfahrung auszuwählen. 
Obwohl es hierzu bislang wenige Untersuchungen gibt, sind Heuristiken aus dem täglichen Organisationsleben als allgegenwärtige Entscheidungsphänomene von Experten/-innen ${ }^{46}$ und Laien nicht mehr wegzudenken. Kanning (vgl. 2011, S. 72) meint: Je kleiner die Organisation sei, desto größer sei die Wahrscheinlichkeit, dass nicht einmal die grundlegendste Expertise über Personalauswahlbefunde bei den Personalverantwortlichen vorhanden sei. Auch wenn bezweifelt werden darf, ob dies an der Größe der Organisation festgemacht werden sollte, zeigt dies, dass im Organisationsverhalten Heuristiken in der Personalauswahl eine tägliche Rolle spielen können aber dafür keine Expertise vorhanden ist. Personalauswahlprozesse sind komplexe Entscheidungssituationen, die auch für erfahrene Recruiter/-innen mit einem begrenzten Informationsvolumen einhergehen. Zum Teil sind die Bewerbungsverfahren mehrstufig und nicht alle Beteiligten in jeder Phase anwesend. Deshalb erhält nicht jeder/jede alle Informationen, sei es über organisa-

46 Dreyfus (2004) differenzieren Expertentum in Novizen (Entscheidung nach definierten Regeln), fortgeschrittener Anfänger/in, Kompetenz, Profi und Experte/-in. 
4.6.1 Heuristiken von Experten/-innen

tionale Bedingungen oder über Informationen, die in der Person des Bewerbenden liegen. Auch Experten/-innen der Personalauswahl müssen deshalb im Entscheidungsprozess auf verkürzte Auswahlstrategien zurückgreifen können (vgl. Koppers 2013, S. 38).

Kanning (2011, S. 232) unterscheidet zwei Grundtypen heuristischen Entscheidens: Eine heuristische Entscheidung basiert auf Fertigkeit und Sachkunde und kann durch regelmäßige Übung erworben werden, z.B. Experten/-innen, wie die Einsatzleitung der Feuerwehr, oder Ärzte/-innen. Bei der anderen heuristischen Entscheidung, die sich nur auf wenige Informationen stützt, wird kein Erfahrungswissen durch Lernprozesse aufgebaut. Diese "Laien-Heuristik" ist besonders anfällig für Urteilsfehler (vgl. Koppers 2013, S. 2), da bei zu wenig Information ein Ausgleich durch heuristische Mechanismen stattfindet, bei dem aber wichtige Informationen wegfallen können, oder es werden falsche Entscheidungsregeln angewandt. Für Letzteres soll folgendes Beispiel die Heuristiken verdeutlichen:

Die Auszubildende $M$ wird nach abgeschlossener Lehre in die Personalabteilung übernommen. Sie übernimmt die 
4.6.1 Heuristiken von Experten/-innen

Aufgabe, die neuen Auszubildenden für das anstehende Lehrjahr auszuwählen. Als Neuling in der Personalauswahl gibt sie sich große Mühe und schaut sich die Unterlagen sehr gewissenhaft an, vergleicht sie mit ihren Aufzeichnungen der Interviews und entscheidet sich für einen Kandidaten mit guten Noten und vielen Praktika. Sie erinnert sich, dass diese Kriterien bei ihrer Auswahl als Azubi damals auch wichtig waren und ihr dies die Lehrer/-innen in der Schule und im Betrieb auch vermittelt haben.

In dem Beispiel setzt die Auszubildende mit wenig Erfahrung in der Personalauswahl einen Fokus auf die Bewerberdetails, aber inr Wissen ist aufgrund fehlender Erfahrung limitiert. Sie greift auf bereits Bekanntes, ihre Erfahrung und organisationsseitige Vorgaben zurück und generiert daraus subjektive Entscheidungsregeln. Weitere Entscheidungskriterien bezieht sie in ihre Personalentscheidung (wie Soft Skills o.Ä.) nicht mit ein. Der andere Grundtyp des heuristischen Entscheidens, der auf Fertigkeiten und Sachkunde basiert, wäre bei der Entscheidung aufgrund seiner Erfahrung anders vorgegangen, z.B. wäre die Auswahl des/der Experten/-in danach ge- 
4.6.1 Heuristiken von Experten/-innen

troffen, was für die Stellenanforderungen nötig gewesen wäre.

Ein/eine Experte/-in kann in der Lage sein aufgrund seines/ihres Erfahrungsschatzes Muster in den Bewerberinformationen zu erkennen, die ihn/sie dazu befähigen, eine Entscheidung zu treffen, die organisationsangemessen und nachvollziehbar sein sollte. Die Entscheidung des/der Experten/-in sollte dabei jedoch nicht allein auf seiner/ihrer subjektiven Gewissheit beruhen, sondern auch verlässliche Indikatoren haben. Koppers (2013, S. 39 f.) stellt dabei die Frage, ob umfangreiches Expertenwissen als Indikator für intuitive Entscheidungsmuster interpretiert werden kann. An diese Frage soll im Folgenden angeknüpft werden.

Entscheidungsregeln der Experten/-innen werden auch von Cognitive Maps (siehe Kapitel 3.3.3.3) beeinflusst und spielen deshalb auch in der Personalauswahl eine Rolle. Wenn es um Entscheidungen in der Gruppe geht, präsentieren diese die durch Lernen und Erfahrung erworbenen kognitiven Muster (Cognitive Maps) des (Organisations)-Wissens der Teilnehmenden (vgl. Koppers 2013, S. 31). Die kognitive Landkarte der Organisation 
4.6.1 Heuristiken von Experten/-innen

stellt für die Gruppenmitglieder ein Bedeutungsschema dar, das sie für die Entscheidungsfindung in der Personalauswahl verwenden. In Bezug auf das Beispiel mit der Auszubildenden $\mathrm{M}$ ist das Bedeutungsschema der Erfahrungen in Betrieb und Schule mit dem Thema Personalauswahl verknüpft. Es führt dazu, dass sich die Auszubildende die Bedeutungsschemata als Entscheidungsregel für ihre eigene Auswahl zunutze macht.

Kognitive Repräsentationen von Experten/-innen sind komplexerer Natur als die von Laien. Experten/-innen wenden auch Entscheidungsregeln an, aber durch bereits erlernte Wahrnehmungs- und Entscheidungsprozesse sind diese evaluierter (vgl. Koppers 2013, S. 31). Denn ihr Erfahrungswissen wurde im Laufe der Zeit durch ihre selbst gestellten Hypothesen in Bezug auf die Personalauswahl überprüft. Dadurch konnten sie sowohl ihr Wissen als auch ihre Wahrnehmung anpassen oder schärfen. Sie greifen, wie ihre Laien-Kollegen/-innen, auch auf Cognitive Maps zurück, sind aber befähigter in der Anwendung. Experten/-innen können dann mit ihren eigenen subjektiven Entscheidungsregeln eine Auswahl treffen, denn ihr Erlerntes unterliegt ihrem eigenen Entscheidungskanon bzw. richtet sich nach den eigenen Hy- 
4.6.1 Heuristiken von Experten/-innen

pothesen. Welche Entscheidungsregeln sie zugrunde legen und im Laufe der Zeit gefestigt haben, hängt mit ihrer Prägung, Sozialisation und ihrem Lernen zusammen.

Experten/-innen benötigen weniger Hinweisreize (sogenannte cues, u.a. Czerlinski, Gigerenzer \& Goldstein 1999, S. 97 f.), um zu einer Entscheidung zu gelangen. Hinweisreize in der Personalauswahl können z.B. Betonungen oder Gewichtungen von Aussagen eines Bewerbers/-in im Vorstellungsgespräch, Beobachtungen in Übungsaufgaben oder der Kleidungsstil sein. Auch diese Expertenentscheidungen können, basierend auf ihrer subjektiven Bewertung der Hinweisreize, heuristisch geprägt sein. Auch Marewski/Gigerenzer (2013, S. 228 f.) sind der Ansicht, dass die Personalauswahl von Experten/-innen heuristisch geprägt ist, um zu einer zeit- und kapazitätssparenden Entscheidung zu kommen, die trotzdem erfolgreich ausfallen kann. Sie schlagen effiziente Entscheidungsbäume (EE) als simple Entscheidungsregeln zur Anwendung von Experten/-innen vor, wie dies Green und Mehr (1997) für die Auswahl von Patienten für eine Herzintensivstation getan haben (siehe 
4.6.1 Heuristiken von Experten/-innen

Grafik in Marewski/Gigerenzer 2013, S. 236). EE dienten in dem Experiment der medizinischen Diagnose bei der Einweisung von Patienten und der Frage, ob diese zur schnelleren (lebensrettenden) Behandlung auf eine Intensivstation sollen. Hierzu sollten die Ärzte der Notaufnahme lediglich drei Fragen beantworten, die ihnen diese Entscheidung schnell und effizient ermöglichen sollte. EE folgen den Regeln der Take-the-best-Heuristik. Es handelt sich quasi um eine Verfahrensanweisung für Organisationsmitglieder und es wird dadurch erneut versucht, die Brücke zwischen normativer Analytik und heuristischen Entscheidungsregeln zu schlagen, indem den Experten/-innen ein Tool an die Hand gegeben wird, um ihre Entscheidungsfindung zu kanalisieren und zu rationalisieren. EE in der Personalauswahl anzuwenden, erscheint aufgrund der Bandbreite an Informationen kaum möglich, denn auch bei der Bewerberwahl gibt es bestimmte Kriterien, die anderen überlegen sind. Das heißt, zum einen würde eine Priorisierung benötigt werden und zum anderen müssten die Gruppenmitglieder sich auf diese Kriterien einigen. Einen Vorteil könnten die Praktiker/-innen darin sehen, dass auch die Bewerberflut durch die Anwendung von EE handhabbarer gemacht wird (z.B. 
4.6.1 Heuristiken von Experten/-innen

cut-offs anhand von gezielten Auslesefragen, wie „Was ist ihre größte Schwäche?"). Es käme einer sehr effizienten Selektion gleich, die aber eher den Versuch unternimmt, anhand der hardfacts der Personalauswahl die Heuristiken einzudämmen. EE sind in der Personalauswahl eher Entscheidungshilfen für Laien, damit diese zu einer angemessenen Entscheidung gelangen können.

Eine Frage, die sich auch Koppers (2013, S. 38 f.) an dieser Stelle stellt, steht im Zusammenhang mit der Verbesserung der Validität im Auswahlprozess und der Nutzung der Expertise versierter Recruitern/-innen. Können Organisationsprozesse in ihrer Validität gesteigert werden, wenn sie von Organisationsmitgliedern „bearbeitet“ werden, die evaluiertes Erfahrungswissen mitbringen? In ihrer Studie stellt Koppers (2013) die These auf, dass Recruiting-Experten/-innen im Laufe ihrer Tätigkeit ein differenziertes kognitives Hypothesen-System erworben haben, das innen ermöglicht, valide eignungsdiagnostische Entscheidungen zu treffen, die sie befähigen, abgekürzte Entscheidungen zu treffen. Heuristiken, die von Experten/-innen angewandt werden, können auch erlernt sein. Dies liegt den Schluss nahe, dass ein Organisati- 
4.6.1 Heuristiken von Experten/-innen

onsmitglied, das über Jahrzehnte Mitarbeitende aus einem speziellen Fachbereich (z.B. IT) auswählt, u.U. schneller zu einer passenden Zielpersonalie gelangt als ein anderer, der für sich zwar eine gute Menschenkenntnis beansprucht, aber wenig Erfahrung mit Einstellungsprozessen und somit in dieser Hinsicht nur wenig Expertise aufweist.

Die kognitiven Lernprozesse und Assoziationen, die Experten/-innen im Laufe ihres Lebens erworben haben, geben ihnen die Gelegenheit, ihr Expertenwissen bei Personalentscheidungen anzuwenden und damit den Entscheidungsprozess heuristisch, aber auf hoch professioneller Ebene zu gestalten (vgl. Koppers 2013, S. 2). Expertise entsteht nicht ausschließlich über diese kognitive und bereichsspezifische Problemlösekompetenz, sondern vor allem auch durch Korrekturwissen, die Reflexion und Adaption des Gelernten. So können Heuristiken entstehen, die zu einem sharing knowledge werden, also zu geteiltem Expertenwissen, dass Experten/-innen weitergeben (vgl. Gatarik/Born 2012, S. 82).

Fifić und Gigerenzer (2014, S. 7) sehen in der heuristischen Personalentscheidung gar eine akkurate Auswahlmethode. Sie ziehen die Schlussfolgerung, dass es 
4.6.1 Heuristiken von Experten/-innen

deshalb möglich sein müsse, die Interviewer/-innen im Auswahlgremium zu reduzieren, wenn doch die Entscheidungen korrekt sein sollen. Forscher/-innen wie auch Organisationen nehmen bei einem Einsatz von mehreren Interviewenden meist an, eine richtige Entscheidung zu treffen (vgl. Weuster 1999a, S. 223). Der Hintergrund für eine solche Sicht- und Vorgehensweise ist die vermeintliche Verbesserung der Objektivität durch mehrere Beobachter, da mehr Interviewer/-innen ihre Eindrücke vergleichen, unterschiedliche Fragen stellen und deshalb mehr Informationen gewonnen werden können. ${ }^{47} \mathrm{Da}$ erscheint es denklogisch nachvollziehbar, dass weniger Interviewer/-innen benötigt würden, da doch die heuristische Entscheidung der Genauigkeit nicht entbehrt. Es würden sich bei der Verwendung von „single interviewers" sowohl die Kosten und Ressourcen reduzieren lassen als auch die sozialen Defekte der Gruppe, wie Free-Riding etc. Allerdings stellt sich die Frage, ob aus diesen Gründen eine Auswahl durch nur einen/eine

${ }^{47}$ Allerdings kann die Schwankungsbreite bei Interviewern/-innen in der Akzeptanz eines/einer Bewerbers/-in zwischen 6 und 56\% liegen (Graves/Karren 1996, S. 165 f., zit. n. Koppers 2013, S. 23). Unterschiede in den Validitäten kommen durch Interviewermerkmale, kognitive Strukturen und den Interviewerkontext zustande. 
4.6.1 Heuristiken von Experten/-innen

Entscheidungsträger/-in vorzuziehen wäre. Bei der Eignungsdiagnose durch mehrere Beurteiler/-innen werden auch meist mehr Anforderungsaspekte beachtet. Schon die Notwendigkeit, eine eigene Entscheidung in einer Konsensdiskussion nachvollziehbar begründen zu müssen, erzeugt ein präventiv selbstkritisches Handeln der Gruppenmitglieder (vgl. Weuster 2012b, S. 66).

Fifić und Gigerenzer (2014) haben in ihrer Studie diese Fragestellung untersucht: „How many interviewers per job applicant are necessary for a company to achieve the highest hit rate?" Sie konnten darstellen, dass Einstellungsgespräche zur Auswahl von Stellenbewerbern/innen, die von zwei Interviewern/-innen geführt wurden, nicht signifikant besser waren als die, die nur von einem/einer Interviewer/-in geführt wurden. Die Untersuchungen ergaben, dass ein/eine einziger/einzige Interviewer/-in gleiche oder bessere Ergebnisse erzielte als mehrere. Sie erklären sich diesen Effekt u.a. mit der Verantwortungsdiffusion der Gruppe (siehe Kapitel 3.4.1). Die einzelnen Teilnehmenden im Bewerberinterview fühlen sich weniger verantwortlich, je größer die Gruppe ist. Der Verantwortungsdiffusion bei Gruppenentscheidungen kann entgegengewirkt werden, indem sich vor der ge- 
4.6.1 Heuristiken von Experten/-innen

meinsamen Entscheidung zunächst jedes Gruppenmitglied unabhängig ein eigenes (schriftliches) Urteil bildet und dieses begründen muss (vgl. Weuster 2012b, S. 55). Mit der Interviewerzahl und der damit zu betrachtenden Validität beschäftigt sich auch Weuster (2012a, S. 223 f.): Auch er fragte sich, ob nicht, wenn doch mehr Interviewende ihre Eindrücke verglichen, mehr Informationen hätten und sich ein Gefühl von Rechenschaft einstelle, die Vermutung naheliege, dass „mehr Interviewer/-innen [...] dem Individuum überlegen“ seien. Eignungsdiagnostisch konnte dies von ihm nicht belegt werden, weder in Bezug auf die Validität noch auf die Messgenauigkeit.

Der "Weniger-ist-mehr-Gedanke“ in der Personalauswahl, nämlich die Interviewenden zu reduzieren, ist für das Organisationsverhalten neu und legt den Fokus auf einzelne, aber qualifizierte Entscheidungsexperten/innen. Deshalb schlagen Fifić und Gigerenzer (2014, S. 7) vor, die Personalauswahl zu verbessern „by training and grooming a single top interviewer rather than investing equally in a team of interviewers“. Zur Möglichkeit der Verbesserung von Personalauswahlentscheidungen schlägt auch Weuster (2012b, S. 54) vor, die Qualifikation der Entscheider/-innen zu verbessern und zusätzlich 
4.6.1 Heuristiken von Experten/-innen

ihre Auswahlentscheidung hinauszuzögern, zu schulen, da die Verzögerung der Entscheidung zu einer valideren Bewertung der Bewerber/-innen führe. Tullar et al. (1979) untersuchten die Besetzung einer Vertriebsstelle, indem sie den Entscheidungszeitpunkt hinauszögerten. In Gesprächen gaben die Gruppenmitglieder differenziertere Bewertungen $a b$, wenn sie bis zur Entscheidung länger warteten (vgl. Tullar et al. 1979, S. 673).

Was bedeutet es nun im Einzelnen, ein/eine Experte/-in zu sein? Gibt es solche überhaupt, wenn Heuristiken die Grundlage für Experten/-innen- wie für Laienentscheidungen sind?

Es sind einige Voraussetzungen vonnöten, um „echtes“ Expertentum zu erlangen:

1. Korrekturwissen: Das Fallwissen von Experten/innen entsteht durch Lernprozesse, wodurch Hinweisreize (cues) schneller erkannt werden. Diese werden von den Experten/-innen reflektiert. Ihre Entscheidungsstrategien sind flexibel an die Umwelt anpassbar. 
2. Sharing Expertise: Heuristiken von Experten/innen können sich im Dialog entwickeln und anpassen (vgl. Gararik/Born 2012, S. 83). Der Austausch des Erfahrungswissens in der Gruppe von Personalverantwortlichen trägt zur Evaluation ihres Wissens bei (z.B. indem sie sich gegenseitig Feedback geben, auf welche Weise die Einstellungsgespräche geführt wurden).

3. Muster erkennen: Auch Simon (1990, S. 9) verweist auf die Wiedererkennbarkeit als Identifikationsmerkmal des Expertentums: „We can regard intuition as a phenomenon that has been rather thoroughly explained: It is achieved through acts of recognition" (Simon 1990, S. 9). Der/die Experte/-in erkennt relevante Hinweisreize (cues) und filtert diese auch aus komplexen Situationen.

4. Heuristische Entscheidungsregeln: Experten/innen nehmen Informationen unbewusst und schnell wahr und verarbeiten sie effizient. Dieser kognitive Prozess läuft häufig nicht nur unbewusst, sondern auch automatisiert ab. Es geht darum, die Kunst zu beherrschen, sich auf die wichtigen Informationen zu konzentrieren, die in einer Ent- 
4.6.1 Heuristiken von Experten/-innen

scheidung verarbeitet werden können, und den

Rest zu vernachlässigen (vgl. Gatarik/Born 2012, S. 83).

An einen/eine Recruiting-Experten/-in sind die gleichen Anforderungen zu stellen. Konkret heißt das, dass die Expertise mit der Erfahrung bei Personalauswahlgesprächen wächst (Fallwissen). Im Auswahlprozess erkennt er/sie Hinweisreize, die für die Stellenauswahl relevant sind, vergleicht diese mit den Anforderungen der Stelle (Anforderungsprofil) und filtert gleichzeitig situationsbeeinflussende Faktoren (wie Wetter, Geräusche, soziale Aspekte) heraus. Der/die Experte/-in ruft dabei vergleichbare Hinweisreize, Situationen, Verhaltensweisen, Stellen oder Bewerberpersönlichkeiten auf, mit denen er/sie gute Erfahrungen gemacht hat. Anhand dieser Informationen können Muster erkannt und in das kognitive System integriert werden (Muster erkennen). „Seine Entscheidungsregeln kann er dann in neuen Kontexten und Situationen mit anderen Bewerbern/-innen überprüfen und evaluieren. So kann er sich über den Zeitverlauf verschiedene stellenrelevante kognitive HypothesenSysteme erarbeiten, die er in jedem neuen Einstellungs- 
4.6.1 Heuristiken von Experten/-innen

interview auf deren Validität prüft und im Gespräch mit dem Bewerber/-innen systematisch abtestet, um zu einer eignungsdiagnostischen Entscheidung zu gelangen“ (Koppers 2013, S. 44).

Wenn alle „Voraussetzungen“ erfüllt sind, scheint es auch in der Personalauswahl ein Expertentum zu geben. Fraglich ist, ob es möglicherweise weitere Aspekte gibt, das Expertentum kritisch zu hinterfragen?

In der Personalauswahl spielen zahlreiche Variablen eine Rolle, insbesondere wenn mehrere Entscheidende die Auswahl treffen. Folgende Fragen könnten dabei weiterführend diskutiert werden:

- Ändern sich im Laufe der Zeit die Entscheidungsregeln? Eine Expertise, die von einem/einer Recruiter/-in im Laufe seines/ihres Arbeitslebens erworben wurde, ist durchwirkt von gesellschaftlichen oder organisationalen Einflüssen. Während in den 1980er-Jahren noch gewisse Vorurteile, wie Geschlechterstereotype, Einfluss in die Personalauswahl nahmen und toleriert wurden, ist heute eine solche Gender-Auswahl politisch höchst bedenklich. Dem trägt auch das AGG (siehe Kapitel 
4.5.2.1) Rechnung. Da sich auch die Anforderungen an Stellen ändern, bekommen die geforderten Kompetenzen im Laufe der Zeit oft auch eine andere Richtung. Diagnostische Instrumente sollen heute vermehrt weiche Faktoren, wie soziale Intelligenz, Flexibilität oder Führungs- und Teamfähigkeit erheben. Insbesondere die Lernfähigkeit tritt bei den geforderten Kompetenzen an die vorderste Stelle (vgl. Naef 2001, S. 117). Wechselnde Anforderungen an die Bewerber/-innen bedeuten also, dass auch die Recruiter/-innen ihre Expertise anpassen müssen. Wenn dieser Weg allerdings nicht beschritten wird, kann nicht von Expertise als flexibler und angemessener Entscheidungsfindung gesprochen werden.

- Sind Entscheidungsregeln explizit zu formulieren? Ist ein/eine Experte/-in ein „Schiffchen im Wind“, der/die seine/ihre Meinung wankelmütig ändert? Im Gegenteil, er/sie könnte Entscheidungsregeln explizit formulieren, die das Grundgerüst seines/ihres Entscheidens ausmachen. Dieses kognitive Gebäude fußt einerseits auf seinem/ihrem 
4.6.1 Heuristiken von Experten/-innen

Wissen ${ }^{48}$ und anderseits auf seiner/ihrer Einstellung, z.B. zur Führungserfahrung (der/die Recruiter/-in definiert zunächst für sich, was gute Führung ausmachen sollte, und legt daraufhin Kriterien der Auswahl fest, z.B. Durchsetzungsfähigkeit).

- Hinterfragen Recruiting-Experten/-innen ihre Entscheidungen? Ob sich Entscheidungsregeln oder die organisationseigenen bzw. individuellen Entscheidungsschemata geändert haben - die Beantwortung dieser Frage bedarf der Selbstreflexion des/der Experten/-in. Dieses Hinterfragen muss kontinuierlich erfolgen.

- Fehlt den Experten/-innen das Feedback? Dem/der Experten/-in gelingt es in der Organisation häufig nicht, seine/ihre Entscheidung zu evaluieren, weil ihm/ihr nicht zurückgemeldet wird, wie der/die eingestellte Bewerber/-in seine/ihre Arbeit macht. Dieses Feedback ist wichtig, um damit die

\footnotetext{
${ }^{48}$ Es kann unterschieden werden zwischen explizitem und implizitem Wissen. Explizites Wissen ist verbalisierbares, objektivierbares, formalisierbares Wissen. Hingegen ist implizites Wissen intuitiver Natur (z.B. Krawattenbinden, Gerüche erkennen, Gleichgewicht halten beim Fahrradfahren) und weniger reproduzierbar.
} 
Qualität seiner/ihrer Entscheidung zu hinterfragen.

Bei nicht bestandener Probezeit (Kündigung) ist dieses Feedback klar und erreicht auch den/die Personalexperten/-in, aber über unklare Passungen (z.B. Person-Organization-Fit) oder Leistungsdefizite erfährt er/sie unter Umständen wenig bis überhaupt nichts. Ob eine Personalentscheidung richtig war, zeigt sich unter Umständen erst nach Jahren (vgl. Femppel 2000, S. 178) Es fehlt daher die Koppelung von Entscheidung und Rückmeldung, d.h., die Teilaktivitäten der Personalauswahl (siehe Kapitel 2.3.2) sind um die Prozesse Umsetzung (d.h. Einstellung des/der Bewerbers/-in und seine/ihre Einarbeitung) und Reflexion gekürzt.

All diese Aspekte zeigen die Schwierigkeit auf, als Experte/-in eine angemessene und objektive Personalentscheidung zu treffen. Die Evaluation der eigenen Erfahrung bzw. des Expertenwissens ist die wichtigste Grundlage für das Expertentum. Koppers (2013, S. 48 f.) kommt deshalb zu dem Schluss, dass Expertise im Personalwesen nur selten aufgebaut wird, weil Recruiting- 
4.6.1 Heuristiken von Experten/-innen

Experten/-innen keine Rückmeldung über die Qualität ihrer Entscheidungen erhalten und ihnen damit die Möglichkeit zur Reflexion fehlt. Ohne Feedback gelingt es innen nicht zu schlussfolgern, an welchen Stellen ihre Entscheidung fehlerhaft war (vgl. Schuler 2002, S. 122). Die Entscheidungsregeln, die der/die Personalexperte/-in sich aufbaut, müssen aber stetig evaluiert und verifiziert werden, um einen angemessenen Lernprozess zu haben. Ohne diesen Zusammenhang ist wahres Expertentum im Personalwesen nicht möglich, und dem Personalauswahlprozess würde eine valide Qualitätskontrolle fehlen. Danach könnten selbst Experten/-innen keine rationalen Entscheider/-innen sein. Unbegrenzte Rationalität scheint deshalb für Experten/-innen auch nicht erreichbar. Aber wer, wenn nicht die Experten/-innen, soll über die Befähigung verfügen, Entscheidungen zu treffen, die sich ohne Defekte darstellen. Diese Ansicht folgt auch der Sichtweise der Befürworter/-innen der fast and frugal heuristics, die die Fähigkeit, die Zukunft zu kalkulieren und zu simulieren, über unbegrenzte Fähigkeiten und Wissen zu verfügen, also das Handeln einer überlegenen menschlichen Rasse, als „dämonisch und traumtänze- 
4.6.1 Heuristiken von Experten/-innen

risch“ kritisieren („demons and nightmares“) (vgl. Gigerenzer/Todd 1999a, S. 33).

Die Organisationen versuchen durch Treppenprozesse, ihr Recruiting mit mehreren Entscheidern/-innen zu verstetigen und qualitativ zu sichern, um die bestmögliche Personalauswahl zu treffen. Weisheiten, wie „Mehr Augen sehen besser" beschreiben die Sichtweise zahlreicher Personalverantwortlicher. Kaum eine Auswahl wird nachhaltig verfolgt (z.B. durch künftige Laufbahnanalyse oder Einarbeitung der Eingestellten) oder organisational hinterfragt (z.B. durch Prozessanalysen, Evaluation). Die Diagnosemethoden könnten dann entsprechend der zu besetzenden Stelle angepasst werden, indem z.B. bei Führungskräften im Vergleich zu Fachkräften im Auswahlprozess eines Assessment-Centers ein stärkerer Fokus auf die Führungskompetenzen gelegt wird, und die Experten/-innen können ihre Einstellungsregeln überdenken.

Grundsätzlich stellt die Person des/der Experten/-in in den meisten Betrachtungen ähnlich dem Konzept der vollständigen Rationalität ein Idealbild dar. Seine/ihre Expertise ist ebenso begrenzt rational, und er/sie handelt genauso heuristisch wie Laien. Der Unterschied liegt in 
4.6.2 Overconfidence bei Experten/-innen

der Geschwindigkeit, mit der eine Entscheidung getroffen wird, und oft auch in der Selbstsicherheit. Zu letztem Aspekt soll eine kurze Betrachtung des OverconfidenceEffektes in Verbindung mit dem Expertentum erfolgen sowie der Frage nachgegangen werden, ob nicht auch die Selbstsicherheit dazu führt, dass Entscheidungen von Experten/-innen einen elementaren heuristischen (Grund-)Charakter haben.

\subsubsection{Overconfidence bei Experten/-innen}

Für gewöhnlich meinen ausgebildete Recruiter/-innen, sie besäßen eine gute Menschenkenntnis und könnten Bewerbereigenschaften sicher einschätzen und Urteile über den Charakter sowie die künftige Performance fällen, weil sie seit Jahren Erfahrung darin hätten sammeln können. Sie gehen davon aus, dass ihre Menschenkenntnis gut ist, d.h., sie beurteilen ihre Entscheidungsfähigkeit qua Erfahrung oder Selbstattest als überdurchschnittlich gut. Der Overconfidence-Effekt besagt, dass Personen mit allzu großer Gewissheit darauf vertrauen, dass das eigene Urteil richtig ist. Eine Person beharrt z.B. auch umso eher auf ihrer Meinung, je besser sie in- 
formiert ist (vgl. Borcherding/Schaefer 1976, S. 54).

Dadurch engen sich die Alternativen ein, und die eigene Unsicherheit wird verringert. ${ }^{49}$ Jemand glaubt sich selbst als Experten/-in auszuweisen, seine/ihre Entscheidung ist aber heuristisch geprägt, weil er/sie dem Glauben anhängt, aus Kompetenz und Erfahrung eine gute Entscheidung treffen zu können. Er/sie entscheidet heuristisch unter Nichtberücksichtigung zahlreicher Informationen.

In einer Studie von Naef (2001, S. 14) gaben Personalverantwortliche an, mit der Qualität ihrer Entscheidungen zufrieden zu sein. In einer Studie mit 1000 befragten Organisationen und deren Personalverantwortlichen gaben 88\% die Qualität der Prognose als „gut" (40\%) oder „ge-

${ }^{49}$ Die Überschätzung könnte sich auf einen fundamentalen Attributionsfehler gründen. Um eine gute und schnelle Personalauswahl zu treffen, wird auf das angenommene gute Urteilsvermögen attribuiert. Bei positiven Erwartungen an die eigene Urteilsfähigkeit in der Personalauswahl werden die Ergebnisse, bei anschließendem Erfolg des/der Bewerbers/-in, auf sich selbst attribuiert und dem „Pluskonto" des Selbstbewusstseins hinzugefügt. Wenn der/die Bewerber/-in scheitert, wird sein/ihr Misserfolg häufiger äußeren Umständen zugeschrieben (vgl. Weuster 2012b, S. 23). Die Fehlannahme, eine gute Menschenkenntnis zu besitzen, lässt den/die vermeintlichen/-e Besitzer/-in glauben, die Personalauswahl abgekürzt anhand weniger Informationen treffen zu können. 
4.6.2 Overconfidence bei Experten/-innen

nügend" (48\%) an. Sind also die Personalverantwortlichen mit ihren Entscheidungen zufrieden? Deshalb bleibt die Frage, ob die vermeintlichen Kenner/-innen wirklich eine angemessene Personalauswahl treffen oder ob es lediglich deren Wille und Vorstellung ist: Geht der/die Experte/-in bei einem Overconfidence-Effekt ein trügerisches Bündnis mit der Heuristik ein? Oder sind Experten/-innen quasi „immun“ und entscheiden immer professionell rational?

Der Overconfidence-Effekt könnte in der Personalauswahl häufig anzutreffen sein, weil sich viele vermeintliche Experten/-innen eine gute "Menschenkenntnis“ attestieren. Wie weit diese Selbstüberschätzung gehen kann, beschreibt Kahneman (2011) in seinem Artikel in der New York Times über seine Auswahlerfahrung bei der Israelischen Armee. Er machte dort seine ersten Erfahrungen mit der Evaluation von Soldaten/-innen, die für eine Führungslaufbahn ausgewählt werden sollten. Er stellte im Laufe der Zeit fest, dass es konkrete Beweise (Führungslaufbahn) dafür gab, dass seine Auswahlmethode nicht funktionierte. Trotzdem hielt er daran fest: „We knew [...] that our predictions were little better than 
4.6.2 Overconfidence bei Experten/-innen

random guesses but we continued to feel and act if each particular predication was valid“" (Kahneman 2011, S. 5). Auch sein Expertengremium unterlag dem Entscheidungsdefekt der Overconfidence. Auf diese Selbsterfahrung gründete sich dann Kahnemans weitere Forschung.

In der Gruppe kann der Overconfidence-Effekt dazu führen, dass Informationen übersehen werden, weil Experten/-innen z.B. glauben, sich auszukennen und deshalb die Entscheidung den anderen Gruppenmitgliedern abnehmen zu können oder ihren Urteilen überlegen zu sein. Ein übertriebenes Selbstbewusstsein der Gruppe kann aber auch zu „harten“ und vorschnellen Urteilen über die Bewerber/-innen führen. Erst eine Kombination von Fertigkeit und Sachkunde mit einer schnellen (intuitiven) Entscheidung macht den Erfolg von heuristischen Expertenentscheidungen aus. Als vorteilhaft können sich heuristische Entscheidungen von Experten/-innen dann erweisen, wenn ihnen ihre langjährige Erfahrung ermöglicht, abgekürzter zu entscheiden, sie aber auch reflektiert und regelorientiert (also auf Basis eines eigenen validierten Regelsystems) damit umgehen. Fifić und Gigerenzer (2014, S. 7) gehen sogar so weit, Heuristiken 
4.6.2 Overconfidence bei Experten/-innen

nicht nur als effiziente, sondern auch als akkurate Verarbeitungsmethode anzusehen. Trotzdem kann ein übertriebenes Selbstbewusstsein Experten/-innen in Gruppen daran hindern, sich professionell mit den Auswahlkriterien der Bewerber/-innen zu beschäftigen. Der Overconfidence-Effekt lässt sie „erblinden“. Die Expertise kann infrage gestellt werden, wenn Experten/-innen in dieses „Unfehlbarkeitsstadium“ eintauchen. ${ }^{50}$ Es konnte untersucht werden, dass sich die Personalentscheidungen erfahrener Interviewer/-innen nicht von den Urteilen der Interviewer/-innen ohne Interviewerfahrung unterscheiden und Erfahrung nur bei hoher Komplexität vorteilhaft ist (Dipboye/Jackson 1999; zit. n. Koppers 2013, S. 50).

Expertentum benötigt eine ständige Evaluierung des Wissens und damit der Entscheidungsregeln der Personalauswahl. Nur wenn sich der/die Personalexperte/-in einerseits selbst hinterfragt und anderseits auch Feedback aus der Organisation erhält, nimmt er/sie die Hürde, um zur validierten Expertise zu gelangen. Fehlt die evaluative Komponente, wird aus dem erworbenen Wissen

${ }^{50}$ Es konnte sogar der Effekt nachgewiesen werden, dass die unbegründete Zuversicht umso größer wird, je schwieriger die Aufgabe ist (vgl. Martin 2012, S. 114). 
4.7 Fazit: Ableitungen für die praktische Personalentscheidung

ein individuelles Regelsystem abgeleitet, das sich durch Wiederholung noch verstärkt (vgl. Koppers 2013, S. 49). Die Folge ist, dass der/die Experte/-in sich immer wieder selbst bestätigt, aber dadurch nicht kompetenter, nur selbstsicherer wird: „Overconfidence arises because people are often blind to their blindness“ (Kahneman 2011, S. 10).

\subsection{Fazit: Ableitungen für die praktische Personalentscheidung}

Nachdem festgestellt wurde, dass auch das Handeln von Experten/-innen heuristisch ist, sollen im folgenden Fazit Ableitungen getroffen werden, was bei einer adäquaten Personalauswahl berücksichtigt werden könnte. Das Fazit setzt sich aus den Zwischenergebnissen der hier untersuchten Kapitel zusammen.

Ausgangspunkt aller Überlegungen ist die in Forschung häufig vertretene These, dass nur bei Überwindung aller Defekte (vgl. Nerdinger/Rosenstiel 2011) und Ausklammerung aller heuristischen Einflüsse eine valide Perso- 
4.7 Fazit: Ableitungen für die praktische Personalentscheidung

$\overline{\text { nalauswahl möglich sei. Schuler (2002, S. 188) gibt einen }}$ Überblick zu den möglichen Fehlern in der Personalauswahl:

- Mangelnder Bezug der Fragen zu den Tätigkeitsanforderungen

- Unzulängliche Verarbeitung der aufgenommenen Informationen

- Geringe Beurteiler-Übereinstimmung

- Dominierendes Gewicht früher Gesprächseindrücke

- Überbewertung negativer Informationen

- Emotionale Einflüsse (z.B. Sympathie) auf die Urteilsbildung

- Beanspruchung des größten Teils der Gesprächszeit durch den/die Interviewer/-in

Der Überblick vermittelt den Eindruck, nur wenn alle Fehler überwunden würden, könne ein halbwegs valides Ergebnis erwartet werden. Die vorliegende Arbeit kommt zu einem anderen Ergebnis, nämlich dass sich die Personalentscheidung nicht auf rationale Entscheidungen 
4.7 Fazit: Ableitungen für die praktische Personalentscheidung

gründen muss, um angemessen und zufriedenstellend (satisfying) zu sein. Hierzu wurden vier Aspekte der Personalauswahl beleuchtet: organisationale Standardisierung und heuristische Auswahl, Wissen und Selbsterkenntnis, Passungen bei der Personalauswahl und Organisationsintegration.

\section{Organisationale Standardisierung und heuristische}

\section{Auswahl}

Die praktische Stärke von Heuristiken anzuerkennen, ist die für die Personalauswahl vielleicht wichtigste Erkenntnis. Heuristiken sind nämlich ein integraler Bestandteil von Entscheidungen. Es gibt zahlreiche Forscher/-innen, die eine personaldiagnostische Entscheidung unter Ausschluss von Heuristiken und Defekten fordern. Gigerenzer und die ABC-Gruppe stellen dieser Sichtweise die hohe ökologische Rationalität von Heuristiken entgegen und messen der heuristischen Entscheidung angemessene Urteile bei. Auch sie raten bei der Entscheidungsfindung zur Akzeptanz von Heuristiken. Andere Forscher/-innen behaupten das Gegenteil, denn gerade in hochanalytischen, rechnerischen Situationen seien sie 
4.7 Fazit: Ableitungen für die praktische Personalentscheidung

untauglich, da sie in die Irre führten, wenn die notwendigen Informationen oder andere Mittel nicht vorhanden seien (vgl. Scholz 1987, S. 91; zit. n. Scholz et al. 2003, S. 16). Analysiert man die Erwägungen, ist nach der hier vertretenen Überzeugung nicht nur die (schlichte) Akzeptanz der elementaren Bedeutung von Heuristiken bei der Personalauswahl einzufordern. Mehr noch ist gar ihr Nutzen hervorzuheben. Dennoch darf man Heuristiken bei der Personalauswahl keinesfalls als unreflektiertes Kriterium über sich ergehen lassen, um nicht aufgrund dessen eine fehlerbehaftete Entscheidung zu treffen.

Daher will diese Arbeit einen vermittelnden Weg einschlagen und beide Standpunkte zusammenbringen. Eine personaldiagnostische Entscheidung ist ohne Heuristiken nicht denkbar. Gleichwohl ist das Einfordern eines Mindeststandards oder einer Mindestvoraussetzung z.B. im Sinne von cut-offs bei der Personalauswahl notwendig, um einer Heuristik nicht bedingungslos zu unterliegen. Gerade hardfacts lassen sich gut in die Personalauswahl integrieren (z.B. Notenauswahl, Anzahl von Berufsjahren, Branchenerfahrung etc.). Wenn diese Voraussetzungen nicht erfüllt sind, wird der/die Bewerber/-in 
4.7 Fazit: Ableitungen für die praktische Personalentscheidung

nicht weiterberücksichtigt (cut-off). Die Personalauswahl sollte nach hier vertretener Meinung eine Kombination aus heuristischer Sichtweise und validen Auswahlmethoden sein. Das eine geht nicht ohne das andere, um der Komplexität personaldiagnostischer Auswahl Herr zu werden. Im Einzelnen:

1. Standardisierte Auswahlmethoden: Um Personalentscheidern/-innen ein Werkzeug an die Hand zu geben, eignen sich standardisierte Diagnosemethoden. Sie nehmen der Entscheidergruppe die Unsicherheit und ermöglichen der Organisation einen roten Faden für gemeinsame Entscheidungen. Leider setzen Organisationen häufig wenig valide Instrumente ein, wie unstrukturierte Interviews oder Bewerbungsmappencheck etc. (vgl. Naef 2001, S. 117). Neben diesen Hindernissen für eine angemessene Personalauswahl entstehen neue: Bei Einstellungsinterviews tritt z.B. auch das Problem der gezielten positiven Selbstdarstellung von Bewerbern/-innen auf. Damit ist auch die Aussagekraft von Personaldiagnostik in Bezug auf die 
4.7 Fazit: Ableitungen für die praktische Personalentscheidung

sozial erwünschte Selbstdarstellung der Bewerber/-innen zu hinterfragen (vgl. Kersting 2008, S. 41). Die Probleme des Einsatzes nicht valider Instrumente sind u.a. unterschiedliche Ergebnisse oder unzutreffende Einschätzungen der Gruppe. In Kapitel 4.4 wurde zur personaldiagnostischen Auswahl die Häufigkeit angewandter Auswahlmethoden und ihrer Validität erörtert. Ein weiteres Argument, warum die Personalauswahl weiterhin mit standardisierten Auswahlmethoden kombiniert werden sollte, ist die Verbreitung und Akzeptanz dieser Verfahren (vgl. Kersting 2008, S. 41). Außerdem können sie auch die Erinnerungsfähigkeit der Gruppenmitglieder fördern, z.B. durch eine exakte Dokumentation. So ist die standardisierte Auswahlmethode geeignet, am Stellenprofil entlang den/die Bewerber/-in eine Mindesthürde nehmen zu lassen oder ihn/sie hieran ggf. auch scheitern zu lassen. Die standardisierte Auswahlmethode sollte somit angewendet werden, um eine weitgehende Validität herzustellen. Sie stellt 
4.7 Fazit: Ableitungen für die praktische Personalentscheidung

eine organisationale Standardisierung der Personalauswahl dar.

2. Heuristische Entscheidungen: Heuristische Entscheidungsregeln in der Personalauswahl hingegen sollen den künftigen beruflichen Erfolg von Bewerbern/-innen quasi intuitiv, d.h. aus dem Bauch heraus, vorhersagen. Die Vertreter/-innen der simple heuristics erheben dabei aber nicht den Anspruch, von einer professionellen Personalauswahl zu sprechen. Sie räumen ihr aber einen großen Stellenwert ein. Die heuristische Auswahl als alleinige organisationale Entscheidungsmethode zu verwenden, erscheint nach der hier vertretenen Ansicht als zwingend fehlgehend. Dennoch bieten Heuristiken ein gutes Unterstützungsniveau und befreien von vielstufigen Prozessen, komplizierten und teuren Diagnoseverfahren.

3. Kombinationsentscheidungen: In der Personalauswahl sind daher sowohl heuristische als auch standardisierte Entscheidungsprozesse vonnöten. Eine Personalentscheidung, die sich einzig auf Gruppenheuristiken gründet, würde u.a. keine or- 
4.7 Fazit: Ableitungen für die praktische Personalentscheidung

ganisationale Akzeptanz erfahren (rein intuitive Entscheidungen hinterlassen bei den Organisationsteilnehmenden oft einen subjektiven Beigeschmack). Eine organisational standardisierte Auswahlmethode, wie die strukturierten Interviews, mit den Methoden heuristischen Entscheidens zu kombinieren, könnte die Vorteile der einen (wiederkehrende Messergebnisse, positive Korrelationen mit dem künftigen Berufserfolg etc.) mit denen der anderen verbinden (schnelles Entscheiden trotz weniger Informationen).

Nach vorliegender Ansicht ist die Bewertung von diagnostischen und heuristischen Entscheidungsverfahren als Kombinationsmodell vor dem Hintergrund personalwissenschaftlicher Betrachtung und Organisationspraxis vielversprechend. Es lässt heuristische Entscheidungen in der Personalauswahl zu, ohne innen aber zu unterliegen, und verteufelt nicht die Intuition der Gruppe, wenn diese eine Entscheidung trifft.

Die gegenwärtigen wissenschaftlichen Bestrebungen tendieren jedoch vermehrt in eine andere Richtung. Sie 
4.7 Fazit: Ableitungen für die praktische Personalentscheidung

fordern gar die Einführung einer DIN-Norm 33430 (u.a. Kersting/Hornke 2003; Kanning 2004; Westhoff et al. 2010) zur Personaldiagnose, um Fehler im Auswahlverfahren dezidiert auszumerzen. Diese „Personaler“-Norm ist eine Prozessvorgabe, die das erklärte Ziel hat, durch ein Verfahren die geeignetsten Mitarbeiter/-innen zu finden (siehe Kanning 2004). Selbstverständlich soll hier nicht verkannt werden, dass der Vorteil von Standardisierungen die Vollständigkeit und Einheitlichkeit der erhobenen Informationen durch vereinheitlichte Durchführung ist, um subjektive Einflüsse und Entscheidungsfehler zu minimieren (vgl. Koppers 2013, S. 16). Aus diesem Grund wird in dieser Arbeit auch befürwortet, einen objektivierten Standardisierungsprozess zu durchlaufen, wie z. B. durch standardisierte Interviews, Leitfäden oder Anforderungsprofile als organisationale Entscheidungshilfen (wie auch in der DIN-Norm gefordert).

Das alleinige Anwenden eines DIN-Norm-basierten Auswahlverfahrens vermag nach unserer Überzeugung nicht, ein taugliches Instrumentarium der Personalauswahl sein, denn ein auf objektiven Kriterien fußender Aus- 
4.7 Fazit: Ableitungen für die praktische Personalentscheidung

wahlprozess lässt das entscheidende Kriterium nämlich völlig außen vor: den Menschen als Subjekt, den Gestik, Mimik und viele andere Parameter auszeichnen, die auch durch eine noch so verdichtete Standardisierung und Vereinheitlichung der Auswahlverfahren mittels DINNorm wahrgenommen und erfasst werden können.

Dieses Ausklammern von „weichen“ Faktoren (soft skills), die mit keiner DIN-Norm in ihrer vollen Ausprägung erfasst werden können, ist ein unzureichender Weg zur Personalauswahl (vgl. Koppers 2013, S. 9). Festzuhalten bleibt, dass die Komplexität der Auswahlsituation von Bewerbern/-innen in Organisationen umfangreich ist und sich daher eine Standardisierung anbietet. Außerdem sollten heuristische Entscheidungsprozesse die Entscheidung begleiten, da ihr Nutzen überwiegt.

\section{Fazit 1: Organisationale Standardisierung und heu- ristische Auswahl}

Ganzheitliche Personalentscheidungen zeichnen sich durch eine Kombination von rational-analytischen und heuristischen Entscheidungsmethoden aus. Personalentscheidungen, die zwar strukturiert und vermeintlich valide 
4.7 Fazit: Ableitungen für die praktische Personalentscheidung

sind, können tatsächlich nicht alle relevanten Entscheidungsparameter, wie Organisationspassung, Eigenschaften oder soziale Kompetenzen darstellen. Durch Gruppeneffekte können Wahrnehmungsverzerrungen sowohl begünstigt (Group-think-Effekt etc.) als auch ausgeglichen werden (z.B. neues Wissen durch die Gruppenmitglieder in der Diskussion). Einem machtbezogenen oder unangemessenen Einfluss anderer Gruppenmitglieder auf die Personalentscheidung kann durch eine strukturierte Vorgehensweise im Bewerberprozess entgegengewirkt werden (z.B. durch einen standardisierten Bewerbungsmappencheck). Nach dieser ersten analytischen Entscheidungsebene sparen heuristische Methoden Zeit und verleihen dem intuitiven Gefühl der Gruppenentscheidung Ausdruck (z.B. der Teamzugehörigkeit). Das Zulassen von Heuristiken stellt den Menschen als Subjekt in den Mittelpunkt der Entscheidung. Die Gruppenmitglieder sollen sich der Existenz von Heuristiken und deren Irrationalität bewusst sein und dürfen dieser nicht per se unterliegen. Ist dies gewährleistet, vermögen Heuristiken einen elementaren Beitrag zur validen 
4.7 Fazit: Ableitungen für die praktische Personalentscheidung

Entscheidungsfindung $\mathrm{zu}$ leisten und einen großen Mehrwert zu liefern.

\section{Wissen und Selbsterkenntnis}

In Kapitel 3.1.4 wurde diskutiert, dass Heuristiken mit Defekten nicht gleichzusetzen seien. Kataloge mit Entscheidungsdefekten (u.a. Martin 2012) vermitteln dem Leser aber oft das Gefühl, dass jede Personalentscheidung defizitär sei. ${ }^{51}$ Dabei muss aber gesehen werden, dass sich auch ein defizitärer Entscheidungsprozess in eine richtige Entscheidung münden kann (siehe Kapitel 4.5.1), nämlich dann, wenn sich der Defekt nicht auswirkt. Dennoch ist das Wissen um Defekte und Heuristiken in der Personalentscheidung (siehe Kapitel 3.4: Verantwortungsdiffusion, Kontrollillusion und eskalierendes Commitment etc.) unverzichtbar. Entscheidungen können von der Gruppe differenzierter und bewusster, ggf. vorsichtiger getroffen werden, wenn sie

${ }^{51}$ Auch Nerdinger und Rosenstiel (vgl. 2011, S. 179) beschreiben die Personalauswahl als fehlerhaft und zu statisch, weil z.B. Veränderungsmöglichkeiten, wie Berufsverläufe, nicht berücksichtigt werden. 
4.7 Fazit: Ableitungen für die praktische Personalentscheidung

um die Fallstricke (wie Heuristiken zu erliegen) weiß, insbesondere weil man bei der Personaldiagnostik potenziell immer von mehr oder minder großen Messfehlern ausgeht (vgl. Kanning 2004, S. 158). Mit dem Wissen um heuristische und defizitäre Fallstricke in der Personalentscheidung kann auch der fehlerhaften Selbstüberschätzung der Gruppe vorgebeugt werden. Gerade vor dem Hintergrund der "guten Menschenkenntnis“, die viele Gruppen und ihre Mitglieder für sich attestieren, scheint es unumgänglich, sich der Fehlerquellen bewusst zu sein (siehe Kapitel 3.4). Durch Bad-practice-Fälle könnte zum Beispiel eine bessere Achtsamkeit gelingen, die die Gruppe zur Vorsicht im Umgang mit Personalentscheidungen anhält.

Für Personalverantwortliche gibt es zahlreiche Weiterbildungen (z.B. personnel and professionell development), die einer kompetenten Personalauswahl Rechnung tra- 
4.7 Fazit: Ableitungen für die praktische Personalentscheidung

gen und auch von heuristischen Elementen geprägt sein können. ${ }^{52}$

Dabei stellt sich die Frage, ob mit einem Fokus auf der Qualifizierung in der Eignungsdiagnostik des/der Einzelnen dem „Effekt der angemessenen heuristischen Entscheidung" Genüge getan wäre. Das würde bedeuten, je besser geschult der/die Experte/-in ist, desto besser sind seine Entscheidungen? Kanning (2004, S. 160) sieht die Lösung in der optimierten Auswahl und Schulung der Diagnostiker. Dieser Gedanke fußt bei inm auf der Sichtweise, dass Personaldiagnostik nur valide Ausprägungen hervorbringen kann, in dem die diagnostischen Ergebnisse in Zahlenwerte transferiert oder zumindest quantitativ messbar gemacht werden (a.a.O.). Dieser Ansatz, die Personalauswahl zu quantifizieren und durch Forderungen, wie die Qualifikation der Auswählenden zu quantifizieren, ist das Leitbild vieler Praktiker und Wissenschaft-

${ }^{52}$ Auch Stephan und Westhoff (2002, S. 16) stellen hierzu Fragen: Was wird in Trainings für Auswahlinterviews gelehrt und gelernt? Wie verhält es sich mit der Divergenz zwischen intuitiver Auswahl und analytischer Diagnostik? Was steht der Nutzung der wissenschaftlichen Erkenntnisse zur Fortbildung der personalverantwortlichen Auswahlgremien entgegen? 
4.7 Fazit: Ableitungen für die praktische Personalentscheidung

ler/-innen, die sich mit der Personalauswahl beschäftigen. Wie in Kapitel 4.5.1 ausgeführt, ist die diagnostische Personalauswahl keine rationale Einbahnstraße, da man die Entscheidung im Nachhinein auch immer reflektieren muss. Es ist daher generell angebracht, die Personalentscheidung mit mehr Wissen anzureichern, um den Horizont der Gruppenmitglieder zu verbreitern.

Selbsterkenntnis: Um Fallstricke bei der Personalentscheidung zu erkennen, ist insbesondere im Gruppengeschehen die Reflexion als Teilaktivität des Entscheidungsprozesses eine wichtige Komponente. Für Gruppen, die mehrstufige Entscheidungen gemeinsam getroffen haben, sind inter- und intragruppale Reflexionsmomente entscheidend, um ihre Selbsterkenntnis zu fördern. Um dies im Gruppengeschehen zu erwirken, bieten sich Methoden, z.B. die außerorganisationale PeergroupBeratung, der organisationale Austausch von Personalverantwortlichen o.Ä., an. Zentrale Bedeutung für das Entscheidungsverhalten in Gruppen hat deshalb u.a. die Selbsterkenntnis der Entscheider/-innen sowohl im Hin- 
4.7 Fazit: Ableitungen für die praktische Personalentscheidung

blick auf Defekte als auch auf heuristische Entscheidungsmuster.

Des Weiteren lassen sich mit dem Wissen um die eigenen Präferenzen und die eigene Persönlichkeit im Entscheidungsverhalten Tendenzen zum eigenen Verhalten in Gruppen ableiten. Präferenzen führen häufig zu einer Musterbildung im Entscheidungsverhalten. Dies gilt auch für Gruppen, die mehrmals gemeinsame Entscheidungen getroffen haben. Umso wichtiger ist, dass die Musterbildung, die die Gruppenteilnehmenden im Laufe ihres Lebens z.B. durch kognitive Lernprozesse und mentale Repräsentationen (Cognitive Maps) erworben haben (siehe Kapitel 3.3.3.3), Entscheidungsregeln bilden, die Informationen nützlich dekodieren oder abstrahieren (vgl. Koppers 2013, S. 31). Hierzu gehören auch der Austausch der Gruppenmitglieder über den Einstellungsprozess und das gegenseitige Feedback. Doch wie gelingt es, dass sich die Gruppenmitglieder passende Schemata zurechtlegen, die für die Personalauswahl angemessen sind, um nicht Opfer der eignen Präferenzen zu werden und um für die zu besetzende Stelle vorbehaltslos zu entscheiden? 
4.7 Fazit: Ableitungen für die praktische Personalentscheidung

Neben der Selbsterkenntnis und dem Recruiting-Wissen (vgl. Koppers 2013, S. 51; Einarbeitung der Recruiter/innen verbessern) ist deshalb die Rückmeldung aus der Organisation über den/die eingestellten Bewerber/-innen ein wichtiger Prozess (vgl. Schuler 2002, S. 122). Miesen et al. (1999, S. 18) erklären sich dazu folgendermaßen: „Sicher spielt die praktische Erfahrung der Diagnostiker eine Rolle. Doch oft fehlen ihnen die notwendigen Rückmeldungen. Sie erfahren zu selten, welche Entscheidung richtig war und welche nicht. Und selbst wenn ein Diagnostiker erfährt, was für Treffer er gelandet hat, weiß er ohne explizites Regelsystem nach ein oder zwei Jahren nicht mehr, welche konkreten Regeln er damals verwendete und heute wieder einsetzen und ausbauen könnte." Das Feedback an die Entscheidergruppe wird benötigt, um die Teilaktivitäten des Personalausprozesses abzurunden und einen Reflexionsprozess in Gang zu setzen.

\section{Fazit 2: Wissen und Selbsterkenntnis}

Lernprozesse von Gruppen gründen sich zunächst auf subjektive Entscheidungsregeln, die entweder organisationsseitig explizit (z.B. durch Einarbeitungsprozesse o- 
4.7 Fazit: Ableitungen für die praktische Personalentscheidung

der Verfahrensanweisungen) oder implizit (z.B. durch Cognitive Maps) weitergegeben werden, d.h., entweder übernehmen Personalgremien diese Entscheidungsregeln oder sie unternehmen die Anstrengung, diese selbst zu formulieren. Die Schwierigkeit besteht darin, das Wissen, auf dem die Entscheidungsregeln basieren, kontinuierlich zu überprüfen. Soweit die Gruppenmitglieder ihr Wissen vertiefen, sollte das nicht nur mit Blick auf die Quantifizierbarkeit erfolgen. Vielmehr ist den Entscheidern/-innen auch das Wissen um Heuristiken näherzubringen, damit sie als Individuum und als Teil einer Gruppe vorbehaltlos in den Entscheidungsprozess eintritt.

Um die Entscheidungen von Personalgremien zu verbessern, ist ein Feedback aus der Organisation über den/die Bewerbers/-in unerlässlich. So kann sich im Nachgang eine möglicherweise stark fehlerbehaftete Entscheidung im Nachhinein doch als richtig erweisen. Wie bereits in Kapitel 4.5.1 dargestellt, wird dieser Schritt durch das Fehlen der Kausalität von Handlungserfolg und Entscheidung durchaus möglich. Outcome-Variablen, die den Handlungserfolg (berufliche Leistung) abbilden könn- 
4.7 Fazit: Ableitungen für die praktische Personalentscheidung

ten, sind z.B. Projekterfolge, Führungsakzeptanz, Teamintegration oder Kundenzufriedenheit (vgl. Koppers 2013, S. 52). Ohne diese Bemühungen der Gruppe, einen Lernprozess anzustrengen, in dem Kausalität aus zusammengetragenem und ausgetauschtem Feedbackwissen entspringt, kann keine Expertise entstehen (siehe Kapitel 4.6.1). Hierzu gehört ebenfalls, dass sich jedes Gruppenmitglied selbst reflektiert, um sich seiner eigenen Entscheidungsregeln bewusst zu werden und diese auch wiedergeben zu können (explizite Entscheidungsregeln). Eine Gruppe kann nur zu einer Expertise-basierten Intuition gelangen, indem der Grundsatz „Lernen durch Evaluation und Reflexion" befolgt wird. Auch wenn viel Erfahrung und Wissen vorhanden sind, muss dieses immer wieder evaluiert und reflektiert werden, um z.B. neue Erkenntnisse aus der Wissenschaft zu berücksichtigen (siehe Kapitel 4.4). Personalentscheider/-innen in Gruppen, die sich selbst kennen, entscheiden differenzierter und ganzheitlicher. Gerade im Hinblick auf Gruppeneffekte sind Verhaltenstendenzen ein wichtiger Indikator und vor allem ein Wissenspool für die Gremiumsmitglieder, 
4.7 Fazit: Ableitungen für die praktische Personalentscheidung

um gemeinsam eine angemessene Personalauswahl zu treffen.

\section{Passungen bei der Personalauswahl berücksichtigen}

Im Kapitel 4.6.1 (Heuristiken von Experten/-innen) wurde die Passung von Organisation und Bewerber/-innen (organization-job-fit oder Organisationspassung) sowie von Bewerber/-innen und Stelle angesprochen (person-job-fit: Kenntnisse, Fähigkeiten und Fertigkeiten). Personalverantwortliche berücksichtigen häufig die Stellenpassung, jedoch weniger die Organisationspassung (vgl. Weuster 2012a, S. 263). Die Organisationspassung bemisst sich nach den vorherrschenden Werten, Normen, Überzeugungen und Denkhaltungen, bezogen auf die Organisation und die Eigenschaften und Werte des/der Bewerbers/in (vgl. auch Kristof-Brown et. al. 2005, S. 285). Organisationspassung ist ein sehr „weiches“ Kriterium und gibt den Gruppenmitgliedern bei der Personalentscheidung wenig greifbare Anhaltspunkte. So kann, neben der Stellenpassung und ähnlicher Kriterien, die Organisationspassung ein Erfolgskriterium für den Organisationserfolg des/der Bewerbers/-in darstellen und umgekehrt. Ein 
4.7 Fazit: Ableitungen für die praktische Personalentscheidung

Beispiel für Organisationpassungen sind eigentümergeführte mittelständische Unternehmen, bei denen unterstellt wird, dass hierfür die Passung besonders wichtig sei (vgl. Martin/Behrends 1998, S. 37) und sich nach den für die Organisation typischen Eigenschaften richten könne (z.B. ein besonderes Commitment dem Produkt gegenüber). Die Einstellung eines/einer Mitarbeiters/-in kann sich auf der Grundlage von Testergebnissen oder anderen Diagnoseinformationen im Nachgang als unpassend herausstellen, weil er/sie unter dem Aspekt der Altersstruktur nicht in die künftige Zusammenpassung passt (vgl. Nerdinger/Rosenstiel 2011, S. 180).

Auch wenn der/die Bewerber/-in fachlich und sozial die Stellenanforderungen erfüllt, bedeutet das nicht gleichzeitig, dass er/sie den „Ton“ der Organisation auch trifft, wodurch eine Einarbeitung sowie die Integration in die Arbeitsgruppe oder das organisationale Gremien erschwert wird oder gar scheitert. Das Gremium der Personalauswahl mag sich dann im Nachgang wundern, warum der/die Bewerber/-in nicht die Probezeit überstanden hat, obwohl die Bewerberanforderungen doch erfüllt waren. 
4.7 Fazit: Ableitungen für die praktische Personalentscheidung

Die Passung zur Organisationskultur und ihren Werten entscheidet nicht nur über die Integration, sondern auch über die Identifikation mit der Organisation und ihren Zielen. Ihre motivatorische Komponente ist deshalb nicht zu unterschätzen. Kristof-Brown, Zimmermann und Johnson (2005, S. 281 f.) konnten in ihrer Metaanalyse zeigen, dass sowohl die Passung zu dem Aufgabenprofil der Stelle als auch die Passung zur Organisation mit der Arbeitszufriedenheit und dem organisationalen Engagement zusammenhängen. Außerdem fanden sie eine negative Korrelation mit der Absicht, das Unternehmen zu verlassen (vgl. Zimmermann und Johnson 2005, S. 299). Auch die Passung von Bewerber/-in und Vorgesetztem/er könnte den Ausschlag für bewerberrelevante Auswahlkriterien geben.

\section{Fazit 3: Passungen bei der Personalauswahl berück- sichtigen}

Passungen sind ein weiteres Kriterium für die Personalentscheidung. Sie spiegeln die Umwelt wider, in der sich neben dem/der Bewerber/-in auch die Gruppe bewegt. Passungen sollen die organisationsindividuelle Umge- 
4.7 Fazit: Ableitungen für die praktische Personalentscheidung

bung, wie kollegiale und vorgesetzte Organisationsmitglieder, sowie die Besonderheiten der Stelle im Zusammenhang mit den Bewerbern/-innen berücksichtigen. Für die Entscheidergruppe sind Passungen wichtig zu berücksichtigen, da sie ein Postentry-Element der Auswahl darstellen, das über Wohl und Wehe der Integration des/der Bewerbers/-in entscheidet und damit für einen Einstellungserfolg ausschlaggebend ist. Die Passung des/der Bewerbers/-in ersetzt dessen/deren Fachkompetenz nicht, sondern steht neben dieser Entscheidungsvoraussetzung als eigenständiges Kriterium und ist von der Gruppe mit Blick auf das Zusammenspiel von Organisation, Arbeitsumfeld (z.B. Abteilung, Vorgesetzten, Kollegen/-innen) oder Stelle zu eruieren.

\section{Organisationsintegration verbessern}

Ein weiteres Postentry-Element (neben der Passung) ist die Einarbeitung des/der Bewerbers/-in (Onboarding). Ist ein/eine Bewerber/-in erst einmal eingestellt, ist die Arbeit der Entscheidergruppe getan. Diese Sichtweise kann sich allerdings schnell als Trugschluss erweisen. Einar- 
4.7 Fazit: Ableitungen für die praktische Personalentscheidung

beitungszeit und -kosten ${ }^{53}$ sind das Investment in die Mitarbeiter/-innen, das verloren geht, wenn er/sie die Organisation verlässt (vgl. Moser/Kraft 2008, S. 110). Dennoch verfahren viele Organisationen nach diesem Schema, und auch die Wissenschaftsliteratur zielt eher auf die Qualität des Diagnoseprozesses bei der Bewerberauswahl. Bestenfalls wird noch die Einarbeitungsschnelligkeit untersucht. Doch das, was sich nach der Einstellung in der Organisation abspielt, sollte an den Entscheidungsprozess gekoppelt werden, z.B. anhand von Rechenschaftspflichten (siehe Kapitel 3.4.1, Verantwortungsdiffusion) oder Rückkopplungen durch ein Feedback an die Auswahlgruppe. Dazu sollte auch gehören, dass der/die Bewerber/-in durch das Auswahlgremium besser über die Stelle informiert wird, weil die Entscheidergruppe aus vorangegangenen Rückkopplungen ihr Wissen über die Stelle angereichert hat. Interviewende, die eine realistische (positive wie negative) Tätigkeitsvorschau geben, können bei Bewerbern/-innen (als Soziali-

${ }^{53}$ Den Kosten für Onboarding können die Kosten der Fehlbesetzung (Produktivitätsverluste, Trennung, Vertretung, Neueinstellung) gegenübergestellt werden (vgl. Jordan 2013, S. 18). 
4.7 Fazit: Ableitungen für die praktische Personalentscheidung

sationsschritt) zu einer Erwartungssenkung (sowie erhöhung) führen, wodurch Enttäuschungen in der Einarbeitungsphase und eine daraus resultierende Frühfluktuation verringert werden können (vgl. Weuster 2012b, S. 131). Neue Mitarbeiter/-innen sollten nicht erst am ersten Tag über Gepflogenheiten informiert werden, genauso wenig, wie die Organisation erst dann mit der Planung zur Einarbeitung beginnen sollte. Jordan (2013, S. 167 f.) schlägt eine Reihe von Einarbeitungsmaßnahmen vor: Einarbeitungsplan erstellen, Wissenslücken schnell schließen, Ziele gemeinsam festlegen, Erwartungen in Jour Fix-Gesprächen transportieren, Feedback über Lernfortschritte geben, eigenes Netzwerk aufbauen etc. Sie können Hilfestellungen sein, wenn das Niveau der Arbeitsleistung zu Beginn nur zu einem gewissen Teil den Erwartungen entspricht. Anpassung und Integration des/der Bewerbers/-in in die Organisation oder Reduktion des Anspruchsniveaus sind mögliche OnboardingSzenarien.

Die Einarbeitung mag nur scheinbar mit der heuristischen Personalauswahl zu tun zu haben. Ihre Betrachtung ist dennoch wichtig, weil bei einer Personalauswahl berück- 
4.7 Fazit: Ableitungen für die praktische Personalentscheidung

sichtigt werden muss, ob man den/die Bewerber/-in am Tag der Einstellung „sich selbst überlassen“ kann oder in die Organisation so ideal wie möglich integriert. Die Passung in die Organisationsstruktur hängt eben nicht nur an "harten“ Merkmalen, wie fachlicher Integrität, Kompetenzen etc., sondern muss antizipiert werden, wie die gesammelten positiven Einstellungsmerkmale durch eine fruchtbringende Einarbeitung noch idealer abgebildet werden können. Die hier vertretene Meinung ist ein Appell an einen heuristischen Personalauswahlprozess, der durch intuitiv-analytische Elemente schlank gehalten werden kann, weil er Gelegenheit bietet, eine fehlende Wunsch-Passung im Nachhinein auszugleichen. Auch wenn der/die Wunschkandidat/-in eingestellt wurde, kann durch eine mangelnde Integration in die Organisation ein "guter" und langer Auswahlprozess konterkariert werden. Die Arbeit präferiert einen schlanken Einstellungsprozess mit heuristischen Elementen in Verbindung mit einer (guten) Integration in die Organisation. 
4.7 Fazit: Ableitungen für die praktische Personalentscheidung

\section{Fazit 4: Organisationsintegration verbessern}

Integraler Bestandteil des Entscheidungsprozesses sollte sein, ob und wie der/die Mitarbeiter/-in nach zuvor bejahter Einstellung auch entsprechend dem Stellenportfolios eingearbeitet werden kann. Vor dem Hintergrund des Fachkräftemangels ist es nicht immer leicht, den/die Wunschkandidaten/-in zu finden. Deshalb kann es lohnen, in Einarbeitung zu investieren, um fehlende Kompetenzen oder fehlendes Wissen auszugleichen. Leitfäden, Weiterbildungen, Neue-Mitarbeiter-Broschüren, Mentoren/-innen etc. sind hierbei hilfreiche und praktische Mittel, die auch kleineren Organisationen zur Verfügung stehen und wenig Anpassung benötigen, wenn sie einmal benannt oder erstellt sind.

Aus den vier Ableitungen für die praktische Personalauswahl zeichnet sich ab, wie die Personalauswahl in Organisationen ergänzt werden kann. Diagnostische Mängel im Entscheidungsprozess, wie geringe Beurteilenden-Übereinstimmung, diverse Einflüsse etc. können zwar nicht behoben werden, aber die Ableitungen sollen 
4.7 Fazit: Ableitungen für die praktische Personalentscheidung

der Praxis eine Hilfestellung zur Reflexion organisationaler Prozesse bieten.

Das erste Fazit schlägt vor, standardisierte und heuristische Auswahlmethoden zu kombinieren. Es dient der Erkenntnis, Heuristiken als natürliche Gegebenheiten in die Personalentscheidung nutzbringend einfließen zu lassen. Im Sinne des zweiten Fazits sollen die Gruppenmitglieder ihre Auswahl kontinuierlich hinterfragen und nach Wissen und Information streben. Das dritte Fazit fordert, dass Passungen bei der Personalauswahl berücksichtigt werden, um eine Abgleichverpflichtung mit allen relevanten Knotenpunkten (wie Stelle, Personen und Organisation) in den praktischen Bewerberprozess $z u$ integrieren. Die Passungen zur Organisation und zur Stelle sollen mehr berücksichtigen als nur tätigkeitsbezogene Merkmale und sollen eine leichtere Integration der Eingestellten ermöglichen. Das vierte Fazit berücksichtigt das Onboarding des/der Bewerbers/-in, d.h., seine Einarbeitung nach der Einstellung, und ist ein Appell, die Einarbeitungsprozesse auszubauen. Um alle Ableitungen im Überblick miteinander zu verknüpfen, soll folgende Abbildung dienen: 
4.7 Fazit: Ableitungen für die praktische Personalentscheidung

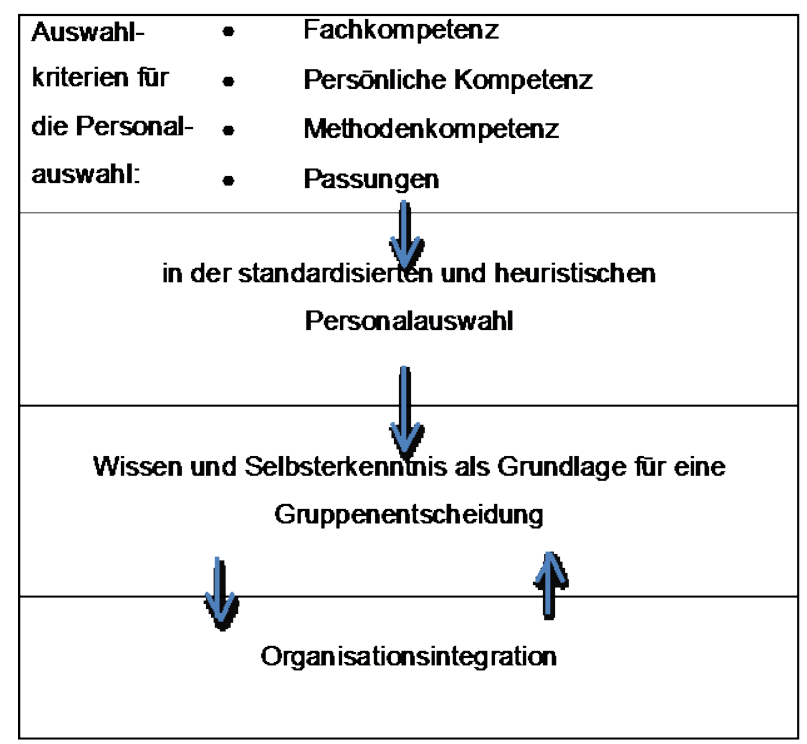

Abbildung 15: Ableitungen für die praktische Personalauswahl als Prozessdarstellung (eigene Darstellung).

In Abbildung 15 sind alle vier Ableitungen relevant für den Auswahlprozess. Die vierte Ableitung („Organisationsintegration“) ist im Anschluss an die Personalauswahl wichtig für ein erfolgreiches Gelingen der Personalauswahl nach dem Auswahlprozess. Die Rückkopplung dient zwar nicht mehr der Korrektur der Fehlentscheidung, ist aber überaus gewinnbringend für die von der Entscheidergruppe zu fällenden nächsten Personalentscheidun- 
4.7 Fazit: Ableitungen für die praktische Personalentscheidung

gen. Die vier praktischen Ableitungen sind Schwerpunkte im organisationalen Entscheidungshandeln, auf die ein Fokus gelegt werden sollte, um heuristische Entscheidungen naturalistisch zu unterstreichen und sich nicht in die Gefahr zu begeben, ein negatives Leistungsfeedback nach der Einstellung zu erhalten. So wird in der Arbeit die Ansicht vertreten, dass sich einfache Heuristiken als Ergänzung zu klassischen (analytischen) Auswahlverfahren anbieten und zugelassen werden sollten, dass sie aber auf ihre Passung im Anwendungsfall hin überprüft werden müssen. Dies sollte sowohl ex post als auch ex ante geschehen. Ziel soll sein, die Intuition der Entscheider/innen sinnvoll und praktisch zu unterstreichen. Dabei muss der Blick aber nicht nur auf die Entscheidung als solche gerichtet werden, sondern auf den OnboardingProzess als zukunftsorientierten Methode des gesamten Personalauswahlprozess. Diese Betrachtung ist vielversprechend und kann die ganze Personalauswahl auf eine andere Entscheidungsgrundlage stellen.

Diese Ableitungen sollen für das Entscheidungsverhalten von Gruppen praktische Erkenntnisse liefern, um die 
Personalauswahl in Organisationen angemessen zu gestalten. Vor allem ging es in der Arbeit darum, den Horizont zu erweitern und nicht nur den Fokus auf die Entscheidung zu legen, sondern sowohl die Prozesse davor als auch danach zu betrachten. Ungeachtet der Organisationsgröße oder der Kapazitäten sind die vier Ableitungen grundlegende Themen für jeden Personalauswahlprozess und auch für jede Organisation anwendbar. Die Integration in den Personalauswahlprozess gestaltet sich einfach, weil es hierzu keiner großen Analysen, Programme oder Projekte bedarf, um sie anzuwenden. Jede Organisation muss für sich selbst definieren, wie sie ihre Prozesse gestalten will. Es war das Ziel der vorliegenden Arbeit, größtmögliche Allgemeingültigkeit für jede Art von Organisation zu erreichen, um den Blick für die Defekte und Heuristiken in der Personalauswahl zu schärfen und in die bestehenden Diagnoseprozesse zu integrieren.

\subsection{Zusammenfassung}

Die Arbeit beschäftigte sich mit Entscheidungen von Gruppenmitgliedern im Rahmen der Personalauswahl. Es konnte festgestellt werden, dass sich auch die Gruppe 
im praktischen Entscheidungsverhalten adaptiv verhält und Heuristiken anwendet. Heuristiken ermöglichen den Gruppenmitgliedern, bei einer komplexen und vielschichtigen Problemstellung handlungsfähig zu bleiben. Sie sind einfache und schnelle Entscheidungsregeln von Gruppenmitgliedern. Dies wurde mithilfe des sozialen Verhaltens von Gruppenmitgliedern untersucht und soll einen Beitrag leisten, um heuristisches Verhalten als Entscheidungshilfe zu verstehen, das in einer sozialen Umwelt eine entscheidende Rolle spielt.

Weil soziale Prozesse einen gemeinsamen roten Faden haben können, gibt es im Entscheidungsverhalten von Gruppen kollektive Bewegungen, die ihre Grundlage im Organisationsverhalten haben. Nicht nur durch Standardisierung greift die Organisation in die Prozesse ein, sondern auch durch die besondere Strukturiertheit kollektiven Entscheidungsverhaltens können Handlungsmuster entstehen. Es wurde dargestellt, dass Heuristiken auf den gemeinsamen Gerüsten der Cognitive Maps der Organisation beruhen und der Gruppe als Handlungsmuster dienen können. Sie stellen dazu die Verbildlichung der Organisationsrealität im Geiste dar und reprä- 
sentieren in der Organisation kollektive Entscheidungsmuster. Deshalb können sie auch ein Gerüst für heuristische Gruppenentscheidungen sein.

Diagnoseprozesse, die von einer rationalen Entscheidungsfindung der Gruppe ausgehen, versuchen heuristische Gefahren im Keim zu erkennen, bevor sie ihren Lauf nehmen. Im Gegensatz dazu verurteilt der Fast-andfrugal-Ansatz Heuristiken nicht als defizitär. Diesem Ansatz konnte in der vorliegenden Arbeit gefolgt werden, da sie nachvollziehbar als Entscheidungshilfe beschrieben werden konnten, denn sie können in einer ökologischen Umgebung durchaus zu angemessenen Ergebnissen führen. Dies konnte anhand des Beispiels der Personalauswahl untermauert werden. Durch die mögliche fehlende Kausalität der Leistung des/der Eingestellten und der Einstellungsentscheidung wurde klar, dass die Entscheidung als solche, wenn sie auf heuristischen Entscheidungsregeln (z.B. Take-the-best-Heuristik) fußt, nicht notwendigerweise defizitär sein muss und auch zu angemessenen Personalentscheidungen führen kann, die sich erst im Laufe der Einstellung zeigen. 
Es wurde analysiert, wie Heuristiken in der Personalauswahl wirken können. Hieraus konnten Ableitungen für die praktische Personalauswahl gefiltert werden. Insbesondere die Personalauswahl von Experten/-innen (z.B. Recruiter/-innen, Personalverantwortliche) wurde hinterfragt. Es konnte festgestellt werden, dass die Heuristiken von Experten/-innen anwendungsorientierter und evaluierter als die von Nicht-Experten/-innen sind. Jedoch erhalten Personalexperten/-innen selten Feedback über ihre Entscheidungen und bemühen sich häufig auch nicht darum. Deshalb basiert ihre erlernte und evaluierte Expertise auf einem Regelsystem, das von ihren eigenen Normen bestimmt wird. Ihre Diagnosemethoden (z.B., wie sie in Einstellungsgesprächen die Bewerber/-innen befragen) sind erlernt, aber werden selten hinterfragt (z.B. durch Gruppenfeedbacks mit anderen Experten/-innen). Sie können deshalb einem Overconfidence-Effekt erliegen, sodass ihre Expertise angezweifelt werden kann, wenn sie sich selbst nicht evaluieren.

Heuristiken bei der Personalauswahl sind nicht frei von systematischen Fehlern, aber sie können dafür erfolgreiche Strategien im Umgang mit Komplexität und Unsi- 
cherheit sein. Es wurde festgestellt, dass der Fehleranfälligkeit dann eine Grenze gesetzt wird, wenn man sich der Heuristik in ihrer diagnostischen Ausprägung und Wirkung bewusst ist. Dann ist das Zulassen von Heuristiken im Rahmen der Personalauswahl nicht nur ein nutzbringendes Begleit-Werkzeug, sondern sogar proaktiv anzuwenden. Sie stellen dabei das „Stammkapital“ dar, das den Entscheidern/-innen zur Verfügung gestellt wird, d.h. das Wissen der Gruppe und die Strukturen der spezifischen Umwelt. Durch ihre ökologische Natur sind sie intuitive Phänomene, die zahlreichen Einflüssen unterliegen und das Entscheidungsverhalten der Organisationsmitglieder naturnah darstellen können.

Diese Meinung steht im Gegensatz zur Forderung nach mehr Standardisierung bei der Personalauswahl, z.B. durch DIN-Normen. Der hier gewählte Ansatz folgt daher einer Kombination aus Standardisierung und einem reflektierten Einsatz von Heuristiken in der Organisationspraxis der Personalauswahl.

Die Fokussierung der Personalentscheidung lässt die Prozessstufen nach der Auswahlentscheidung außer Acht: Der/die eingestellte Bewerber/-in wird allzu oft sich 
selbst überlassen. Aber gerade die Postentry-Elemente, wie Organisationsintegration und Einarbeitung, Feedback an die Recruiting-Experten/-innen sowie Reflexion der Personalauswahl, sind bislang, wenn überhaupt, nur stiefmütterlich in Organisationen vorhanden. Sie sollten nach dem hiesigen Forschungsergebnis einen höheren Stellenwert genießen, denn diese Postentry-Elemente können für eine kommende Personalentscheidung maßgeblich sein. Für weitere Forschungsvorgaben in der Individual- oder Gruppenpsychologie kann gerade diese Kombination einen hohen praktischen Nutzen haben.

Eine Herausforderung der heuristischen Bewertung wird in der Praxisarbeit sein, das generelle Wissen über Entscheidungen und ihre Prozesse in den Organisationsalltag bei der Personalauswahl zu integrieren. Folgt man den hier gebildeten Ableitungen, muss das Dogma der rationalen Entscheidungsfindung als vermeintlicher Heilsbringer für Organisationen für beendet erklärt werden. Das hehre Ziel der Rationalität bleibt durch das tatsächliche Entscheidungsverhalten der Organisationsmitglieder keine zwingende Voraussetzung für einen angemessenen Entscheidungsprozess. Denn auch, wenn 
man beabsichtigt, unter vollständiger Beleuchtung und Auswertung aller relevanten Informationen der Teilnehmenden oder Umweltgegebenheiten eine richtige Entscheidung zu treffen, werden sich Entscheidungsdefekte im Entscheidungsprozess nicht ausschließen lassen. Entscheidungssituationen, ihre Einflüsse und die Organisationsdynamiken sind intern wie extern so komplex, dass weder Anleitungen, Analysen noch wissenschaftliche Erkenntnisse es vermögen, die Qualität einer Entscheidung zielsicher und positiv zu kanalisieren.

Die Arbeit geht deshalb zusammenfassend nicht von einer Verteufelung von Entscheidungseffekten wie Heuristiken aus, sondern sieht darin durchaus einen Gewinn (u.a. Kreativität, Wechselwirkung als Gewinn der Gruppe, schnelles Entscheiden etc.). Hierbei soll aber nicht außer Acht gelassen werden, dass natürlich eine (vorgelagerte) Standardisierung zwingend ihren Platz im Rahmen einer jeden Personalauswahl hat. Die positive Ergänzung durch analytische und heuristische Bewertungsparameter scheint ein sinnvoller Weg zu einer angemessenen Entscheidung zu sein. 


\section{Abbildungsverzeichnis}

Abbildung 1: Abgrenzung von normativer und deskriptiver Entscheidungstheorie (in Anlehnung an Wossidlo 1975, S. 104).

Abbildung 2: Teilaktivitäten im kollektiven

Entscheidungsprozess (eigene Darstellung). 62 Abbildung 3: Beispiel einer heuristischen Schlaufe (eigene Darstellung).

Abbildung 4: Kausale Beziehung zwischen Entscheidung und Handlungserfolg im rationalen bzw. heuristischen Entscheidungsprozess (eigene Darstellung).

Abbildung 5: Formen der Rationalität, in Anlehnung an:

Gigerenzer und Todd (1999, S. 7). 162 Abbildung 6: Erweiterte Entscheidungsregeln heuristischen Verhaltens durch soziale Prozesse (eigene Darstellung).

Abbildung 7: Entstehung von Gruppenheuristiken durch soziale Prozesse (eigene Darstellung).

Abbildung 8: Kontrollillusion (eigene Darstellung). 228 Abbildung 9: Elemente des eskalierenden Commitments (eigene Darstellung). 235 
Abbildung 10: Einordnung Personalauswahlsituation, in Anlehnung an Grünig und Kühn (2012, S. 225).

Abbildung 11: Einflüsse auf die Entscheidungssituation „Personalauswahl“ der Gruppe (eigene Darstellung). 256

Abbildung 12: Fokus der Arbeit auf Beurteilung und

Einstellungsauswahl bei der

Personalentscheidung (eigene Darstellung). 294

Abbildung 13: Beispiel für einen heuristischen

Fehlschluss von einer Information auf eine Prognose (eigene Darstellung). 306

Abbildung 14: Kategorisierung von Heuristiken (in

Anlehnung an Gigerenzer und Todd (1999b, S. 359).

325

Abbildung 15: Ableitungen für die praktische

Personalauswahl als Prozessdarstellung (eigene Darstellung). 407 


\section{Literaturverzeichnis}

$\begin{array}{ll}\text { Abel, B., Wittgen, R. } & \text { Problemorientiertes Informati- } \\ \text { (1977). } & \text { onsverhalten: Individuelle und } \\ & \text { organisatorische Gestaltungs- } \\ & \text { bedingungen innovativer Ent- } \\ & \text { scheidungssituationen. Darm- } \\ & \text { stadt: Toeche-Mittler. }\end{array}$

Ackoff, R.L. (1961). Systeme, Organisation und interdisziplinäre Forschung. In: E. Witte \& A.L. Thimm (Hrsg.) (1977). Entscheidungstheorie. München: Gabler Verlag, S. 274-289.

Ahrens, H.J. (1976). $\quad$ Urteilsdiskrepanzen bei der Optimalitätseinschätzung von Gruppenentscheidungen. In: E. Brandstätter \& H. Schuler (Hrsg.). Entscheidungsprozesse in Gruppen. Bern: Huber, S. 2745.

Argyris, C., Schön, D.A. Die lernende Organisation: (2006). Grundlagen, Methode, Praxis (3. 
Aufl.). Stuttgart: Klett-Cotta.

Auer-Rizzi, W. (1998). Entscheidungsprozesse in

Gruppen: Kognitive und soziale

Verzerrungstendenzen. Wies-

baden: Deutscher Universitätsverlag $\mathrm{GmbH}$.

Bamberg, G., Betriebswirtschaftliche EntCoenenberg, A.G. scheidungslehre (14. Aufl.). (2008). München: Vahlen.

Bazerman, M.H. (1998). Judgment in managerial decision making (4. Aufl.). New York: Wiley.

Becker-Beck, U., Eskalierendes Commitment bei Wend, D. (2008). Gruppenentscheidungen: Begünstigende Faktoren und Maßnahmen zur Reduktion. In: Gruppendynamik und Organisationsberatung. Wiesbaden: VSVerlag, S. 238-256.

Behrends, T., Personalstrategien in kleinen Jochims, T. (2006). und mittleren Unternehmen. In: A. Martin (Hrsg.). Managementstrategien von kleinen und mitt- 
leren Unternehmen. Stand der theoretischen und empirischen Forschung. München [u.a.]: Hampp, S. 145-168.

Behrens, H. (1980). Politische Entscheidungsprozesse: Konturen einer politischen Entscheidungstheorie. Opladen: Westdt. Verlag.

Bender, K. (1957). Die Führungsentscheidung im Betrieb. Stuttgart: C.E. Poeschel Verlag.

Bickle, G. (2011). Intelligenz, Persönlichkeit und Selbstdarstellung: Aktuelle Perspektiven der Personalpsychologie. In: P. Gellerie \& C. Winter (Hrsg.). Potenziale der Personalpsychologie. Göttingen [u.a.]: Hogrefe, S. 113-120.

Bierhoff, H.-W. (2000). Sozialpsychologie: ein Lehrbuch (5. Aufl.). Stuttgart: Kohlhammer.

Boos, M. (1996). Entscheidungsfindung in Grup- 
pen: Eine Prozessanalyse. Bern [u.a.]: Huber.

Boos, M., Scharpf, U. Eine Methode zur Analyse von Fisch, R. (1991). Interaktionsprozessen beim Problemlösen und Entscheiden in Sitzungen. In: Zeitschrift für Arbeits- und Organisationspsychologie, 35(3), S. 115-121.

Borcherding, K., Urteilsdiskrepanzen bei der OpSchaefer, R. (1976). t timalitätseinschätzung von Gruppenentscheidungen: In: $\mathrm{H}$. Brandstätter \& H. Schuler (Hrsg.). Entscheidungsprozesse in Gruppen. Bern: Huber, S. 4763.

Brandstätter, E., Entscheiden unter Risiko. In: J. Hertwig, R., Behnke. et al. (Hrsg.). Jahrbuch Gußmack, M. (2010). für Entscheidungstheorie. Band 6: Neuere Entwicklungen des Konzepts der Rationalität und ihre Anwendungen. Wiesbaden: Springer, S. 101-124. 
Brettschneider, V.

Brinkmann-Herz, D. (1972).

Bronner, R. (1973).

Bronner, R. (1989).
(2000).

Entscheidungsprozesse in Gruppen: theoretische und empirische Grundlagen der Fallstudienarbeit. Bad Heilbrunn: Klinkhardt.

Entscheidungsprozesse in den Aufsichtsräten der Montanindustrie: eine empirische Untersuchung über die Eignung des Aufsichtsrates als Instrument der Arbeitnehmermitbestimmung. Berlin: Duncker \& Humblot.

Entscheidung unter Zeitdruck: eine Experimentaluntersuchung zur empirischen Theorie der Unternehmung. Tübingen: Mohr.

Planung und Entscheidung: Grundlagen, Methoden, Fallstudien. München [u.a.]: Oldenbourg.

Bronner, R., Appel, W., Empirische Personal- und Or- 
Wiemann, V. (1999). ganisationsforschung. Grundlagen, Methoden, Beispiele. München [u.a.]: Oldenbourg.

Cascio, W.F. (1987). Applied psychology in personnel management (3. Aufl.). Englewood Cliffs: Prentice-Hall.

Cascio, W.F., Die Messung des finanziellen

Reynolds, R.H. (2011) Nutzens in der Personalauswahl. In: P. Gelleri \& C. Winter (Hrsg.). Potenziale der Personaldiagnostik, S. 59-71.

Cerny, M., Decision making in human reGluckaufova, D. (1993). sources management. In: Central European Journal for Operations Research and Economics, 2(2), S. 161-175.

Chaiken, S. (1980). Heuristic vs. systematic information processing and the use of source vs. message cues in persuasion. In: Journal of Personality and Social Psychology (45), S. 524-537. 
Chaiken, S., Trope, Y. Dual-process theories in social (1999). psychology. New York [u.a.]: Guilford Press.

Choo, C.W. (1998). The knowing organization. New York [u.a.]: Oxford Univ. Press.

Cohen, M. D., A garbage can model of orgaMarch, J.G., nizational choice. In:

Olsen, J. P. (1972). http://www.unc.edu/ fbaum/teac hing/articles/Cohen_March_Ols en_1972.pdf, Stand: 14.10.2015.

Cyert, R.M., Eine verhaltenswissenschaftliMarch, J.G. (1995). che Theorie der Unternehmung (2. Aufl.). Stuttgart: SchäfferPoeschel.

Cyert, R.M., Simon, H. A Behavioral Theory of the Firm. (1963). Malden (Mass.): Blackwell.

Czerlinski, J., How good are simple heuristics?

Gigerenzer, G., In: G. Gigerenzer \& P.M. Todd Goldstein, D. (1999). (Hrsg.) Simple heuristics that make us smart. Oxford: University Press, S. 97-118. 
Davis, J.H. (1969).

Davis, J.H. (1992).

Drewes, S. (2013).

Drewes, S., Schultze, T., Schultz-Hardt, S. (2011).
Group performance. London:

Addison-Wesley Publishing

Company.

Some Compelling Intuitions about Group Consensus Decisions. Theoretical and Empirical Research and Interpersonal Aggregation Phenomena. In: Organizational Behavior and $\mathrm{Hu}$ man Decision Processes, Vol. 52, S. 3-38.

Gruppenleistung und Gruppenlernen bei der Steuerung dynamischer Systeme. Göttingen: Univ. Diss.

Leistung in Gruppen. In: D. Frey \& H.-W. Bierhoff (Hrsg.). Sozialpsychologie - Interaktion und Gruppe, S. 222-244. 
Dreyfus, S.E. (2004). The Five-Stage Model of Adult Skill Acquisition. In:

http://www.bumc.bu.edu/facdevmedicine/files/2012/03/Dreyfusskill-level.pdf, Stand:

20.07.2015.

Eagly, A.H., Chaiken, S. Process theories of attitude for(1993). mation and change: The elaboration likelihood and heuristicsystematic models. In: A.H. Eagly \& S. Chaiken (Hrsg.). The psychology of attitudes. Orlando: Harcourt Brace, S. 303-350.

Eisenführ, F., Rationales Entscheiden (5.

Weber, M., Langer, T. Aufl.). Berlin: Springer.

(2010).

Eisenhardt, K.M., Strategic Decision Making. In: Zbaracki, M. (1992). Strategic Management Journal, Band 13, S. 17-32.

Engels, W. (1962). Betriebswirtschaftliche Bewertungslehre im Licht der Entscheidungstheorie. Köln: Westdt. Verlag. 
Femppel, K. (2000). Das Personalwesen in der deutschen Wirtschaft: eine empirische Untersuchung. In:

http://hdl.handle.net/10419/1173 63, Stand: 09.02.2015.

Festinger, L. (1957). A Theory of Cognitive Dissonance. Stanford, CA: Stanford University Press.

Fifić, M., Gigerenzer, G. Are two interviewers better than (2014). one? In: http://www4.gvsu.edu/FIFICM/a ssets/publications/2014FificGige renzer.pdf, Stand: 01.06.2015.

Fischer, D. (1994). Gestaltung wissensbasierter Systeme auf der Grundlage betrieblicher Entscheidungssituationen. Göttingen: Unitext-Verlag.

Fischer, R. (1993). Argumentation und Entscheidung: zur Idee und Organisation von Wissenschaft. München [u.a.] : Profil-Verl.

Fleischer, H. (2001). Einflussstrukturen und ihre Be- 
deutung für die Entscheidungsfindung in Problemlösungsgruppen. München: Rainer Hamp Verlag.

Fleischhut, N. (2013). Moral judgment and decision making under uncertainty. Berlin: Humboldt-Universität Berlin.

Forster, A. (2013). Availability Heuristic. In: http://www.leuphana.de/fileadmi n/user_upload/Forschungseinric htun-

gen/imf/files/lexikon/wahrnehmu ng/Availability_Heuristic.pdf, Stand: 03.10.2015.

Frey, D., Bierhoff, H.-W. Sozialpsychologie (2011). Interaktion und Gruppe. Göttingen [u.a.]: Hogrefe.

Frey, D., Ochsmann, R. Attraktivitätsveränderung der (1978). Entscheidungsalternativen als Möglichkeit der Reduktion kognitiver Dissonanz. In: M. Irle (Hrsg.). Attraktivitätsbeurteilung von Alternativen. Zeitschrift für 
Sozialpsychologie (4), S. 88100.

Gatarik, E., Born, R.

Sharing Expertise als Kern von (2012). Wissensmanagement. Wiesbaden: Gabler Verlag.

Gerlach, A. (2008). $\quad$ Entscheidungsdefekte als Barrieren für Nachhaltigkeitsinnovationen. München: Rainer Hampp Verlag.

Gigerenzer, G. (2000). Adaptive Thinking. Oxford [u.a.]: Oxford Univ. Press.

Gigerenzer, G. (2004). Fast and frugal heuristics: The tools of bounded rationality. In: D. Koehler \& N. Harvey (Hrsg.). Handbook of judgment and decision making. Malden: Blackwell, S. 62-88.

Gigerenzer, G. (2007). Bauchentscheidungen: die Intelligenz des Unbewussten und die Macht der Intuition. München: Bertelsmann.

Gigerenzer, G., Homo Heuristicus: Why Biased 
Brighton, H. (2009).

Minds Make Better Inferences.

Topics in Cognitive Science,

1(1), S. 107-143.

Gigerenzer, G.,

Denken und Urteilen unter Unsi-

Gaissmaier, W. (2006). cherheit: Kognitive Heuristiken.

In: J. Funke (Hrsg.). Enzyklopä-

die der Psychologie: Denken

und Problemlösen. Bd. 8. Stutt-

gart: Hogrefe, S. 329-374.

Gigerenzer, G., Why Heuristics Work. In:

Gaissmaier, W. (2011). http://gthb.usc.edu/Events/Giger en-

zer\%20\&\%20Gaissmaier\%20\% 282011\%29\%20Heuristic\%20D ecision\%20Making.pdf, Stand: 01.10.2015.

Gigerenzer, G., Reasoning the Fast and Frugal Goldstein, D. (1996). Way: Models of Bounded Rationality. In:

http://www.dangoldstein.com/pa pers/FastFrugalPsych

Review.pdf, Stand: 01.11.2015.

Gigerenzer, G., Entscheiden. In: W. Sarges 
Marewski, J.N. (2013). (Hrsg.). Management-

Diagnostik. Göttingen: Hogrefe,

S. $228-241$.

Gigerenzer, G.,

Bounded rationality: the adapti-

Selten, R. (2001).

ve toolbox. Cambridge, Mass.

[u.a.]: MIT Press.

Gigerenzer, G.,

Fast und frugal heuristics: the

Todd, P.M. (1999a).

adaptive toolbox. In: G. Gige-

renzer \& P.M. Todd (Hrsg.).

Simple heuristics that make us

smart. Oxford: University Press,

S. 3-36.

Gigerenzer, G.,

What we have learned so far. In:

Todd, P.M. (1999b).

G. Gigerenzer \& P. M. Todd

(Hrsg.). Simple heuristics that

make us smart. Oxford: Univer-

sity Press, S. 357-365.

Greitemeyer, T.,

Preference-consistent evaluati-

Schulz-Hardt, S. (2003). on of information in the hidden profile paradigm: Beyond grouplevel explanations for the dominance of shared information in group decisions. In: Journal of 
Personality and Social Psychology, 84, S. 322-339.

Grünig, R., Kühn, R. (2012).

Hauschildt, J., Gemünden, H.-G., Grotz-Martin, S., Haidle, U. (1983). Heintzeler, R. (2009).

Hell, B., Päßler, K. (2011).

Hinrichs, D. (2011).
Entscheidungsverfahren für komplexe Probleme: Ein heuristischer Ansatz (4. Aufl.). Berlin [u.a.]: Springer Gabler.

Entscheidungen der Geschäftsführung - Typologie, Informationsverhalten, Effizienz. Tübingen: Mohr/Siebeck.

Strategische Frühaufklärung im Kontext effizienter Entscheidungsprozesse. München [u.a.]: Hampp.

Eignungsdiagnostik im Dienste der Berufsberatung oder der Personalauswahl? In: P. Gelleri \& C. Winter (Hrsg). Potenziale der Personaldiagnostik, S. 9-21.

Sequenzielle Entscheidungen 
Hinsz, V.B.,

Tindale, R.S.,

Vollrath, D.A. (1997).

Höpfner, R.G. (1997). unter Unsicherheit: Erkenntnisse aus einem Experiment zum Confirmation Bias. Hamburg: Verlag Dr. Kovac.

The emerging conceptualization of groups as information processors. In: Psychological Bulletin (121), S. 43-64.

Die Auswahl des Personalleiters als Entscheidungsproblem.

München u.a.: Hampp.

Huffcutt, A., Arthur, W. Hunter and Hunter (1984) revisi(1994). ted: Interview validity for entrylevel jobs. In:

http://maamodt.asp.radford.edu/ PSYC\%20651/Huffcutt\%20\&\%2 OArthur\%20(1994)\%20Interview. pdf, Stand: 14.04.2015.

Insko, C., Schopler, J. Experimental social psychology: (1972). text with illustrative readings. London, New York: Academic Press.

Janis, I.L. (1972). Victims of groupthink: A psycho- 
logical study of foreign-policy decisions and fiascoes. Boston: Houghton Mifflin.

Janis, I.L. (1982). Groupthink: psychological studies of policy decisions and fiascoes. Boston [u.a.]: Houghton Mifflin.

Janis, I.L. (1989). Crucial decisions: Leadership in policymaking and crisis management. New York: Macmillian.

Jarz, E. (1997).

Entwicklung multimedialer Systeme: Planung von Lern- und Masseninformationssystemen. Wiesbaden: Dt. Univ. Verlag.

Jochims, T. (2010).

Personalpolitik in mittelständischen Unternehmen: personalpolitische Konfigurationen und organisationale Dissonanz. München [u.a.]: Hampp.

Joost, N. (1975).

Organisation in Entscheidungs- 
prozessen. Tübingen: J.C.B.

Mohr Verlag.

Jordan, U., Külpp, B., $\quad$ Das erfolgreiche EinstellungsinBruckschen, I. (2013). terview. Wiesbaden: Springer. Jungermann, H. (2009). Entscheiden aus psychologischer Sicht. In: H. Jungermann \& C. Lütge (Hrsg.). Entscheidung und Urteil, Band 8. Göttingen: Vandenhoeck \& Ruprecht.

Jungermann, $\mathrm{H}_{\text {, }}$ Die Psychologie der EntscheiPfister, H.-R., dung: Eine Einführung (3. Aufl.).

Fischer, K. (2010). Heidelberg: Spektrum Verlag. Kahle, E. (2000). Wie liest man eine Cognitive Map? Fragen und Hypothesen zur Sinngebung und Sinnvermittlung in Organisationen. Unveröffentliches Manuskript der Universität Lüneburg.

Kahneman, D. (2006). Choices, Values and Frames. D. Kahneman \& A. Tversky (Hrsg.) 
(2006). Cambridge: Cambridge University Press.

Kahneman, D. (2011). Don't Blink! The Hazards of Confidence. In:

http://www.nytimes.com/2011/10

/23/magazine/dont-blink-the-

hazards-of-

confidence.html?_r=0, Stand:

15.08.2015

Kahneman, D., Conditions for intuitive expertise:

Klein, G. (2009).

A failure to disagree. In:

http://www.hansfagt.dk/Kahnem an_and_Klein\%282009\%29.pdf, Stand: 12.03.2015.

Kahneman, D., Judgment under Uncertainty. In:

Tversky, A. (1974). http://statweb.stanford.edu/ cga tes/PERSI/Courses/Phil166266/TverskyK-

HeuristicsBiases.pdf, Stand: 05.08.2015.

Kahneman, D., Prospect Theory: An analysis of Tversky, A. (1979). Decision under Risk. In: Eco- 
nometrica, Vol. 47 (No. 2), S.

263-291.

Kanning, U.P. (2004). Standards der Personaldiagnostik. Berlin [u.a.]: Beuth.

Kanning, U.P. (2011). Förderung der wissenschaftlich fundierten Personaldiagnostik in der Praxis. In: P. Gelleri \& C. Winter (Hrsg.). Potenziale der Personaldiagnostik. Göttingen: Hogrefe, S. 71-83.

Kanning, U.P. (2012). O Organisationspsychologie. Göttingen [u.a.]: Hogrefe.

Kaplan, R.E., Whatever it takes: decisionmaMcCall, M. (1985). $\quad$ kers at work. Englewood Cliffs: Prentice-Hall.

Kerschreiter, R. (2003). Soziale Informationsvalidierung und Informationsbewertung als Ursachen für den Diskussionsvorteil geteilter und präferenzkonsistenter Informationen in Gruppen: eine kritische Über- 
prüfung und Revision des

"Mutual-enhancement"-Modells.

München: Univ. Diss.

Kerschreiter, R.,

Informationsaustausch in Ent-

Mojzisch, A.,

scheidungsprozessen. In: T.

Schulz-Hardt, S.,

Stumpf. Teamarbeit und Tea-

Brodbeck, F. (2003).

mentwicklung. Göttingen:

Hogrefe, S. 85-113.

Kersting, M. (2008).

Qualität in der Diagnostik und

Personalauswahl - der DIN-

Ansatz. Göttingen [u.a.]: Hogre-

fe.

Kersting, M.,

Qualitätssicherung und -

Hornke, L.F. (2003). $\quad$ optimierung in der Diagnostik:

die DIN 33430 und notwendige

Begleit- und Folgeinitiativen.

Psychologische Rundschau, 54

(3), S. 175-184.

Kingdon, J.W. (1995). Agendas, Alternatives and

Public Policies (2. Aufl.). New

York: Harper/Collins.

Kirsch, W. (1970).

Entscheidungsprozesse, 1.

Band: Verhaltenswissenschaftli- 
che Ansätze der Entscheidungstheorie. Wiesbaden: Gabler Verlag.

Kirsch, W. (1971).

Entscheidungsprozesse, 3.

Band: Entscheidungen in Organisationen. Wiesbaden: Gabler Verlag.

Kirsch, W. (1974). Betriebswirtschaftslehre: Systeme, Entscheidungen, Methoden. Wiesbaden: Gabler Verlag.

Kirsch, W. (1988). Die Handhabung von Entscheidungsproblemen: Einführung in die Theorie der Entscheidungsprozesse (3. Aufl.). München: Kirsch.

Koppers, N. (2013). Z Zu den Determinanten von analytischen und intuitiven Urteilsund Entscheidungsprozessen von Recruitern in Einstellungsinterviews. In: http://wwwbrs.ub.ruhr-unibochum.de/netahtml/HSS/Diss/Ko 
ppersNadja/diss.pdf, Stand: 23.09.2015.

Krings, A. (1971). Betriebliche Entscheidungen bei unvollkommener Voraussicht: eine Anwendung auf die Personalpolitik. In: Zeitschrift für Betriebswirtschaft: Ergänzungsheft; 97 (3H.). Wiesbaden: Gabler Verlag.

Kristof-Brown, A.L., Consequences of Individual's fit Zimmermann, R.D., at work: a meta-analysis of perJohnson, E.C. (2005). son-job, person-organisation, person-group, and personsupervisor fit. Personnel Psychology, 58(2), S. 281-342.

Krüger, D. (2006). Veränderungsprozesse in der Arbeits- und Personalpolitik vor dem Hintergrund der demographischen Entwicklung: Handlungsansätze für die betriebliche Praxis. Kassel: Kassel Univ. Press.

Liebau, E. (1979). $\quad$ Organisation und Entscheidung. 
Kritische Analyse der Theorie der Unternehmungsorganisation. Frankfurt am Main [u.a.]: Campus-Verlag.

Lindstädt, H. (1997).

Optimierung der Qualität von Gruppenentscheidungen: ein simulationsbasierter Beitrag zur Principal-Agent-Theorie. Heidelberg: Physica Verlag.

Luhmann, N. (2000). Organisation und Entscheidung. Opladen [u.a.]: Westdt. Verl.

Lüthgens, C. (1996). Wo Janis irrte: eine kritische Betrachtung zentraler Randbedingungen für Groupthink unter besonderer Berücksichtigung der Dissonanztheorie. Kiel: Univ. Diss.

Lyding, L. (2010).

Kognitive Heuristiken vom Typ Take-The-Best als Trainingstool für komplexes Problemlösen am Beispiel von Personenauskunftshotlines. Heidelb. Univ. D. Lyles, M.A. (1981). $\quad$ Formulating Strategic Problems: 
Empirical Analysis and Model Development, Strategic Management Journal (Vol. 2), S. 61-75.

March, J.G. (1990).

Entscheidung und Organisation: kritische und konstruktive Beiträge, Entwicklungen und Perspektiven. J.G. March (Hrsg). Wiesbaden: Gabler Verlag.

March, J.G. (1994). A primer on decision making. How decisions happen. New York: Simon \& Schuster.

March, J.G., Organizations. New York: Wiley.

Simon, H. (1958).

Martin, A. (1995).

Führungsstrukturen und Entscheidungsprozesse. In: http://www.leuphana.de/fileadmi n/user_upload/Forschungseinric htungen/imf/files/schriftenreihe 11_Fuehrungsstrukturen_und Entscheidungsprozesse.pdf, Stand: 05.10.2015.

Martin, A. (1996). Die Erklärung der Personalpoli- 
tik. In:

http://www.leuphana.de/fileadmi n/user_upload/Forschungseinric htungen/imf/files/schriftenreihe/5_Di e_Erklaerung_der_Personalpolit ik.pdf, Stand: 23.08.2015.

Martin, A. (1998). Affekt, Kommunikation und Rationalität in Entscheidungsprozessen: Ergebnisse eine Studie über den Einfluss von Gruppenstrukturen auf das Problemlösungsverhalten. München: Hampp.

Martin, A. (2001).

Kommunikation und Entscheidungsprozesse. In:

http://www.leuphana.de/fileadmi n/user_upload/Forschungseinric htungen/imf/files/schriftenreihe/ 2_Kommunikation_und_Entsche idungsprozesse_.pdf, Stand: 04.11.2015.

Martin, A. (2004). Strategisches Unternehmens- 
verhalten aus normativer und diskriptiver Sicht. In:

http://www.leuphana.de/fileadmi n/user_upload/Forschungseinric htungen/imf/files/schriftenreihe/

17_Strategisches_Unternehmen sverhalten_aus_normativer_und _deskriptiver_Sicht.pdf, Stand: 03.11.2015.

Martin, A. (2012).

Fehlentscheidungen: Warum wir tun was wir später bereuen.

Darmstadt: WBG.

Martin, A., Behrends, T. Organisationsstrukturen als De(1998). terminanten des Entscheidungsprozesses in mittelständischen Unternehmen. In:

http://www.leuphana.de/fileadmi n/user_upload/Forschungseinric htungen/imf/files/schriftenreihe/ 9_Organisationsstrukturen_als_ Determinanten_des_Entscheidungsprozess es_in_mittelstaendischen_Unter 
nehmen.pdf, Stand: 03.11.2015.

McKenna, F.P. (1993). It won't happen to me: Unrealistic optimism or illusion of control?, British Journal of Psychology 84 (1), S. 39-50.

Miebach, B. (2012). Organisationstheorie (2. überarb. und erweiterte Aufl.). Wiesbaden: Springer.

Miesen, J., ELIGO: Eine vorläufige Antwort Schuhfried, G., auf Grundprobleme der testgeWottawa, H. (1999). $\quad$ stützten Eignungsdiagnostik. In: Wirtschaftspsychologie, 6 (1), S. $16-24$

Mintzberg, H. (2003). The strategy process (4. Aufl.). Upper Saddle River, NJ: Pearson Education.

Mintzberg, H. (2010). Managen. Offenbach: Gabal Verlag.

Mojzisch, A. (2009). Der Einfluss von Entscheidungspräferenzen auf die Informationsverarbeitung bei Gruppenentscheidungen und Neuro-kognitive Grundlagen 
sozialer Interaktionen: Begleitpapier zur kumulativen Habilitation. Göttingen: Univ. Habil.Schr.

Moser, K., Hahn, T.,

Galais, N. (2000).

Moser, K., Kraft, A. (2008).

Mullins, L. (2010).
Naef, B. (2001).

Expertentum und eskalierendes Commitment. In: Gruppendynamik und Organisationsberatung, 31(4), S. 439-449. Eskalierendes Commitment gegenüber Mitarbeitern: Ein Rahmenmodell. In: Gruppendynamik und Organisationsberatung. Wiesbaden: VS-Verlag, S. 107121.

Management and Organizational Behaviour (9. Aufl.). Harlow [u.a.]: Financial Times Prentice Hall.

Wissenschaftliche Theorie und Anforderungen der Praxis in der Personalselektion. Zürich: Zentralstelle der Studentenschaft.

Neumer, J. (2009). Gemeinsame Entscheidungsfin- 
dung: Perspektiven, Ansatzpunkte und blinde Flecken. In: http://www.isf-muenchen.de/pdf/ Expertise-Gemeinsame\%20 Entscheidungsfindung.pdf, Stand: 26.11.2013.

Nutt, P.C. (1984).

Types of organizational decision processes. In: Administrative Science Quarterly (29), S. 414450.

Olson, M. (2004).

Die Logik des kollektiven Handelns (3. Aufl.). Tübingen: Mohr Siebeck.

Peterreins, H. (2008). Grundsätze soliden Investierens. Wiesbaden: Gabler Verlag.

Peters, J. (1980).

Das Aussagensystem der Wohlstandsökonomik bei Marktunvollkommenheit in Form externer Effekte: ein Beitrag zur Theorie der kollektiven wirtschaftlichen Entscheidung. Würzburg [u.a.]: Physica-Verl. 
Pfeiffer, F. (2006).

Pikkemaat, B. (2002)

Presser, G. (2002).

Regnet, E. (2001).

Eskalierendes Commitment und präferenzkonsistente Informationsbewertung: Der Umgang mit Expertenmeinungen bei zweifelhaftem Entscheidungserfolg. In: https://www.deutsche-digitalebibliothek.de/binary/KIYDXBUALS2J XDA42507TWMOB66NCQMA/f ull/1.pdf, Stand: 04.11.2015. Informationsverhalten in komplexen Entscheidungssituationen. Dargestellt anhand der Reiseentscheidung. Frankfurt: Peter Lang.

Lazy-decision making: Entscheiden durch zielgerichtetes Präzisieren der Wahrscheinlichkeitsinformation. Dortmund: Univ. Diss. Konflikte in Organisationen (2. Aufl.). Göttingen: Hogrefe Verlag. 
Reimer, T., Hoffrage, U. Models of Bounded Rationality: (2004). The Approach of Fast and Frugal Heuristics. In: Management Revue, 15 (2004), H. 4, S. 437 459.

Reimer, T.,

Entscheidungsheuristiken in

Hoffrage, U.,

Gruppen. In:

Katsikopoulos, K.

http://web.ics.purdue.edu/ -treim

(2007).

er/Reimer_et_al_2007.pdf

30.05.2015.

Reimer, T.,

The use of recognition in group

Katsikopoulos, K.

(2004).

decision-making. In:

http://onlinelibrary.wiley.com/sto

re/10.1207/s15516709cog2806_

6/asset/s15516709cog2806_6.p

$d f ? v=1 \& t=i 82$ na3tr $\& s=e 0$ daf9a5

e10d5957a1ce7ebd6807a791f4

463d34, Stand: 01.11.2015.

Reina, L. (2005).

From subjective expected utility theory to bounded rationality: an experimental investigation on categorization processes in integrative negotiation, in commit- 
tees, decision making and in decisions under risk. Göttingen: Univ. Verlag.

Riesenhuber, M. (2006).

Roberts, $\mathrm{H}$., Weick, K.E. (1993).

Rosenstiel, L. von (1992).

Rosenstiel, L. von, Nerdinger, F.W. (2011). psychologie: Basiswissen und

und Eskalation. Wiesbaden:

Univ.-Verl.

Collective Minds in Organizations: Heedful Interrelating on Flight Decks. In: Administrative Science Quarterly (38. Jg.), S. 357-381. In:

http://www.Ise.ac.uk/study/exec utiveEducati-

on/customisedExecutiveEducati on/INAP/Collectivemionnd.pdf, Stand: 09.07.2015.

Grundlagen der Organisationspsychologie: Basiswissen und Anwendungshinweise (3. erw. Aufl.). Stuttgart : SchäfferPoeschel. Grundlagen der Organisations- 
Anwendungshinweise (7. überarb. Aufl.). Stuttgart: SchäfferPoeschel.

Sader, M. (2000).

Psychologie der Gruppe (7.

Aufl.). Weinheim [u.a.]: Juventa-

Verlag.

Salinger, E. (2003).

Betriebswirtschaftliche Ent-

scheidungstheorie: Einführung

in die Logik individueller und kollektiver Entscheidungen (5.

Aufl.). München [u.a.]: Olden-

bourg.

Sanders, K., Kianty, A. Organisationstheorien: Eine Ein(2006).

führung. Wiesbaden: GWV

Fachverlage $\mathrm{GmbH}$.

Sarges, W. (2008). Innovative Ansätze für die Eignungsdiagnostik. W. Sarges \&

D. Scheffer (Hrsg.). Göttingen [u.a.]: Hogrefe.

Sauermann, J. (2012). Ticken Gruppen anders? Ein Laborexperiment zur unterschiedlichen Motivation von Individuen und Gruppen in Ver- 
handlungen. In: Jahrbuch für Handlungs- und Entscheidungstheorie. Wiesbaden: VS Verlag für Sozialwissenschaften, S. 81104.

Schäfer-Pietig, R. (1995).

Schauenburg, B. (2004).

Scholl, W. (1995).

Scholz, R.W., Mieg, H.A., Weber O. (2003).
Suboptimale Gruppenentscheidungen: Untersuchungen zur Informationsnutzung und Entscheidungsgüte. Erlangen, Nürnberg: Univ. Diss. Motivierter Informationsaustausch in Gruppen: Der Einfluss individueller Ziele und Gruppenziele. Göttingen: Niedersächsische Staats- und Universitätsbibliothek.

Lehrbuch der Organisationspsychologie. In: Schuler, H. (Hrsg.) (3. vollst. erw. und überarb. Aufl.). Bern: Huber. S. 515-556. Wirtschaftliche und organisationale Entscheidungen. In: http://e-collection.library.ethz.ch/ 
eserv/eth:26174/eth-26174-

01.pdf, Stand: 19.05.2015.

Schuler, H. (1993)

Personalauswahl im europäischen Vergleich. In: E. Regnet \& L.M. Hofmann (Hrsg.). Personalmanagement in Europa. Göttingen: Verlag für Angewandte Psychologie, S. 129-139.

Schuler, H. (2000). Psychologische Personalauswahl: Einführung in die Berufseignungsdiagnostik (3. Aufl.). Göttingen: Verl. für Angewandte Psychologie.

Schuler, H. (2002). Das Einstellungsinterview. Göttingen: Hogrefe.

Schuler, H. (2007). Handbuch der Arbeits- und Organisationspsychologie. $\mathrm{H}$. Schuler \& K. Sonntag (Hrsg.). Göttingen [u.a.]: Hogrefe.

Schuler, H. (2014). Lehrbuch der Personalpsychologie (3. überarb. und erw. 
Aufl.). Göttingen [u.a.]: Hogrefe.

Schuler, H., Hell, B., Trapmann, S.,

Schaar, H., Boramir, I. (2007)

Schultze, T.,

Mojzisch, A.,

Schulz-Hardt, S. (2012). ment tasks: Group-to-individual transfer as an alternative. Organizational Behavior and Human Decision Processes, 118, S. 2436.

Schulz-Hardt, S. (1997). Realitätsflucht in Entscheidungsprozessen: von Group- 
think zum Entscheidungsautismus. Bern [u.a.]: Huber.

Schulz-Hardt, S. (2002). Entscheidungsprozesse in Gruppen. In: E. Witte (Hrsg.). Sozialpsychologie wirtschaftlicher Prozesse: Beiträge des 17. Hamburger Symposions zur Methodologie der Sozialpsychologie. Lengerich [u.a.]: Pabst.

Schulz, D.H. (1977). Die Initiative zu Entscheidungen . Tübingen: Mohr

Schüßler, T. (1993). Entscheidungsunterstützungssysteme für betriebliche Gruppenentscheidungen. Neuwied: Rasstein AG.

Sherif, M., Sherif, C. Groups in harmony and tension. (1953). New York: Harper \& Row.

Simon, H.A. (1959). Theorien der Entscheidung in den Wirtschafts- und Verhal- 
tenswissenschaften. In: E. Witte \& A.L. Thimm (Hrsg.) (1977).

Entscheidungstheorie. Wiesbaden: Gabler Verlag, S. 82-108.

Simon, H.A. (1965). The shape of automation: for men and management. New York [u.a.]: Harper \& Row.

Simon, H.A. (1977). Models of discovery and other topics in the methods of science. Boston: Reidel \& Dordrecht.

Simon, H.A. (1993). $\quad$ "Homo rationalis": die Vernunft im menschlichen Leben. Frankfurt/Main [u.a.]: Campus-Verl.

Simon, H.A., Organisation und Individuum. March, J.G. (1976). Menschliches Verhalten in Organisationen. Wiesbaden: Gabler Verlag.

Skinner, E.A. (1996). $\quad$ A guide to constructs of control. In: Journal of Personality and 
social psychology (54), S. 549570

Stahlmann, M., Wendt-Kleinberg, W. (2008).

Stasser, G., Titus, W. (1985).

Staw, B. (1997).

Staw, B., Ross, J. (1987).
Zwischen Engagement und innerer Kündigung: fortschreitender Personalabbau und betriebliche Interaktionskulturen. Münster: Verl. Westfälisches Dampfboot.

Pooling of unshared information in group decision making: Biased information sampling during discussion. In: Journal of Personality and Social Psychology (48), S. 1467-1478.

The escalation of commitment: An update and appraisal. In: Z. Shapira (Hrsg.), Organizational decision making. Cambridge, S. 191-215.

Behavior in escalating situations. In: B. Staw (Hrsg.). Research in organizational behavior: an annual series of analytical 
essays and critical reviews.

Greenwich, CT: JAI Press, S.

12-47.

Stein, F.A. (1990).

Betriebliche Entscheidungssituationen im Laborexperiment:

die Abbildung von Aufgaben-

und Struktur-Merkmalen als Va-

liditätsbedingungen. Frankfurt am Main [u.a.]: Lang.

Steiner, I.D. (1972). Group Process and Productivity.

Experimentelle Untersuchungen zum Verhalten in Gruppen. New York [u.a]: Acad. Press.

Stephan, U., Personalauswahlgespräche im Westhoff, K. (2002). Führungskräftebereich des deutschen Mittelstandes: Bestandsaufnahme und Einsparungspotenzial durch strukturierte Gespräche. In: http://tudresden.de/die_tu_dresden/ fakultaeten/fakultaet_mathematik_ und_naturwissenschaften/fachri 
chtung_psychologie/fachrichtun g/institute/publikationen_karl_we sthoff/118.pdf, Stand:

03.10.2015.

Stocké, V. (2002)

Framing und Rationalität: die Bedeutung der Informationsdarstellung für das Entscheidungsverhalten. München [u.a.]: Oldenbourg.

Strobel, A. (2004)

Diagnoseinstrument zur Erfassung der Interviewerkompetenz in der Personalauswahl. Frankfurt a. M.: Pearson.

Stürmer, S., Sozialpsychologie der Gruppe.

Siem, B. (2013). München [u.a.]: Reinhardt.

Tajfel, H., Turner, J.C. The social identity theory of in(1986). tergroup behavior. Monterey, Calif.: Brooks/Cole, S. 7-24.

Thompson, J.D. (1967). Organizations in Action: social science bases of administrative 
theory. New York [u.a.]: Mc Graw-Hill.

Tullar, W.L.,

Mullins, T.W.,

Caldwell, S.A. (1979)

Tversky, A.,

Kahneman, D. (1983). ing: The conjunction fallacy in probability judgment. In: Psychological Review (91), S. 293315.

Tversky, A.,

Kahneman, D. (1986). $\quad$ ming of Decisions. In: Journal of Business (59), S. 251-278.

Weber, W. (1994). Betriebliche Bildungsentscheidungen: Entscheidungsverläufe und Entscheidungsergebnisse. München [u.a.]: Hampp.

Weinert, A.B. (2004). Organisations- und Personalpsychologie: Lehrbuch (5. 
überarb. Aufl.). Weinheim [u.a.]: Beltz.

Wessler, M. (2012). Entscheidungstheorie. Wiesbaden: Gabler Verlag.

Westhoff, K.,

Hagemeister, C.,

Kerstin, M., Lang, F.,

Moosbrugger, $\mathrm{H}$.,

Reimann, G.,

Stemmler, G. (2010).

Weuster, A. (2012a). Personalauswahl I: Internationa-

Weuster, A. (2012b) le Forschungsergebnisse zu Anforderungsprofil, Bewerbersuche, Vorauswahl, Vorstellungsgespräch und Referenzen (3. überarb. Aufl.). Wiesbaden: Gabler Verlag.

Grundwissen für die berufsbezogene Eignungsbeurteilung nach DIN 33430 (3. Aufl.).

Lengerich: Pabst Science Publishers.

Personalauswahl II: Internationale Forschungsergebnisse zum Verhalten und zu Merkmalen von Interviewern und Bewerbern (3. überarb. Aufl.). Wiesbaden: Gabler Verlag. 
Witte, E., Hauschildt, J., Innovative EntscheidungsproGrün, O. (1988). Z Z zesse: die Ergebnisse des Projektes "Columbus". E. Witte, J. Hauschildt, O. Grün (Hrsg.). Tübingen: Mohr.

Wittenbaum, G.M., Mutual enhancement: Toward Hubbell, A.P., Zuckeran understanding of the collectiman, C. (1999). ve preference for shared information. In: Journal of Personality and Social Psychology, Vol. 77(5), S. 967-978.

Woike, J.K. (2008). Die paradoxe Rationalität einfacher Heuristiken. Bochum: Univ. Verlag.

Wossidlo, P.R. (1975). Zum gegenwärtigen Stand der empirischen Entscheidungstheorie aus mikroökonomischer Sicht. In: H. Brandstätter \& B. Gahlen (Hrsg.) Entscheidungsforschung. Tübingen: Mohr, S. 98-124. 